3x.

(9)
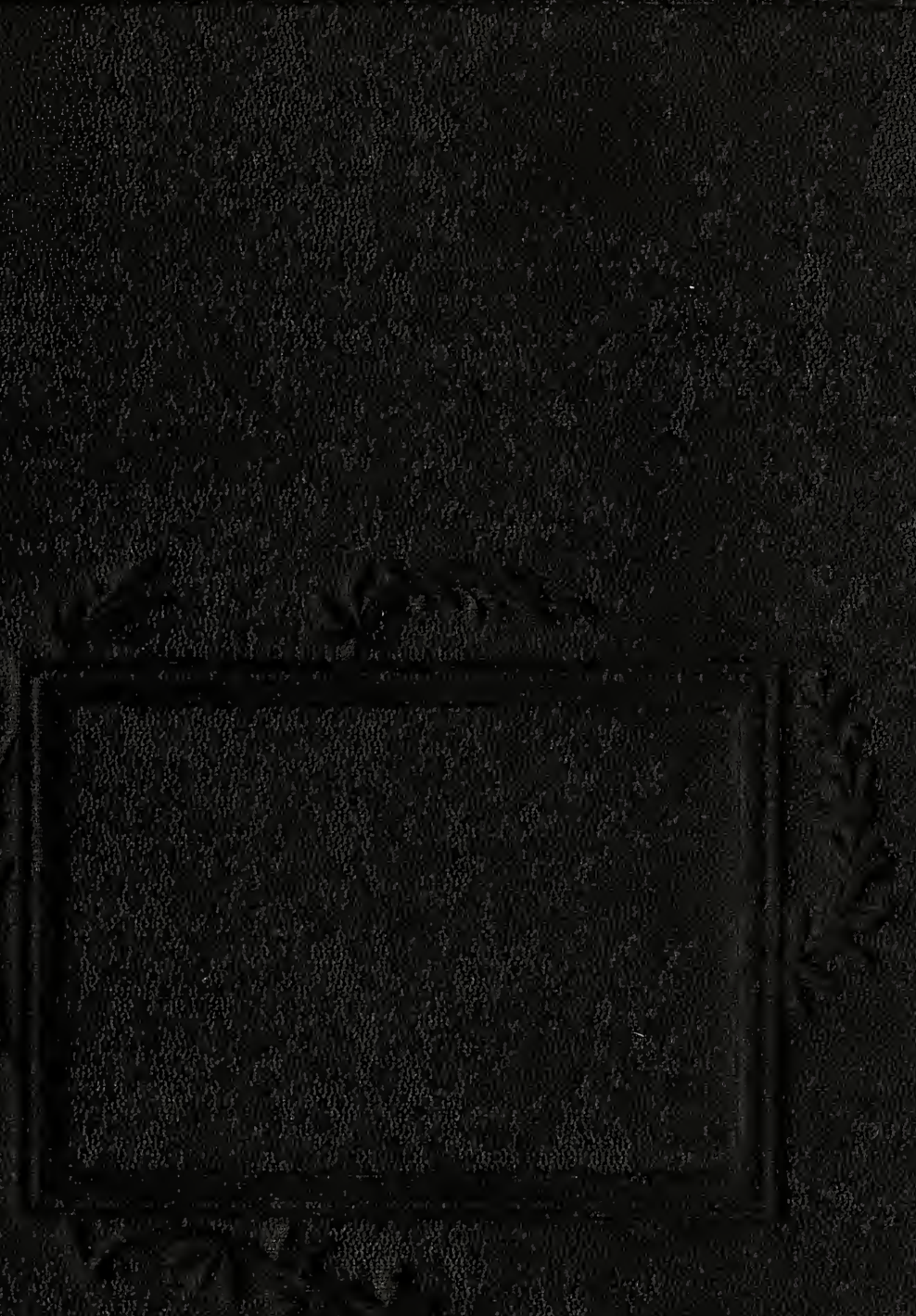

3.
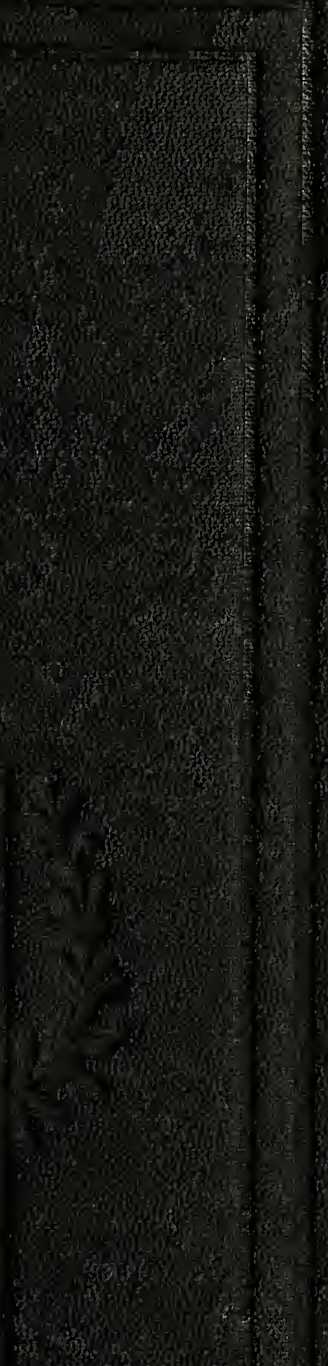

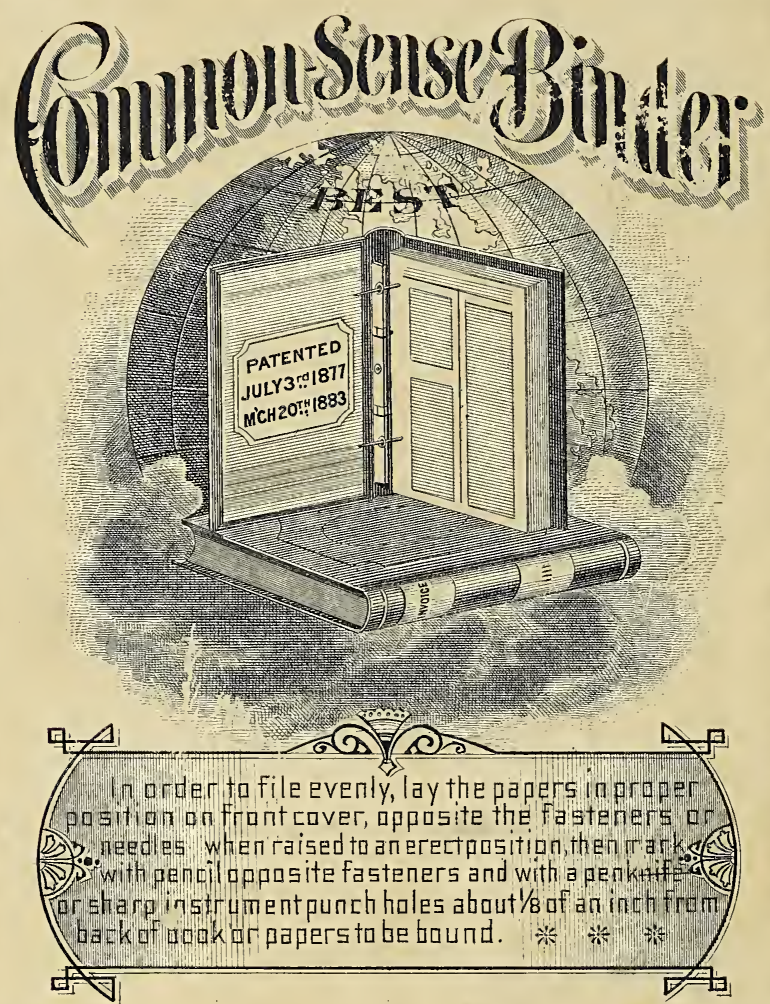

\section{Asal. Shipmanis Sons, MANUFACTURERS NETYYOLIK.}


508

VoL. II

6. De Annezaga

Viaggio

bireum navigazione

Della

Regia bouvetta

baracciolo

$$
1881-84
$$

2

Poma

1885

U.S.

National

Llessecan 

$-1-$

si 



\section{VIAGGIO DI GIRGUUNAVIǴAZIONE}

DELLA

REGIA CORVETTA “ CARACCIOLO " 



\section{VIAGGIO \\ DI \\ CIRCUMNAVIGAZIONE \\ DELLA}

\section{REGIA CORVETTA "CARACCIOLO»}

(COMANDANTE C. DE AMEZAGA)

NEGLI ANNI 1881-82-83-84

VOLUME II.

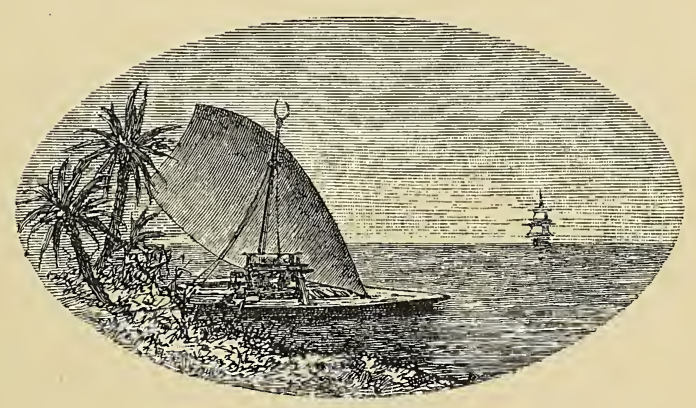

ROMA

FORZANI E COMP., TIPOGRAFI DEL SENATO

1885 

AL MIO MAESTRO

\section{SIMONE PACORET DI SAINT-BON}

VICE AMMIRAGLIO

TRIBUTO DI AFFETTO 



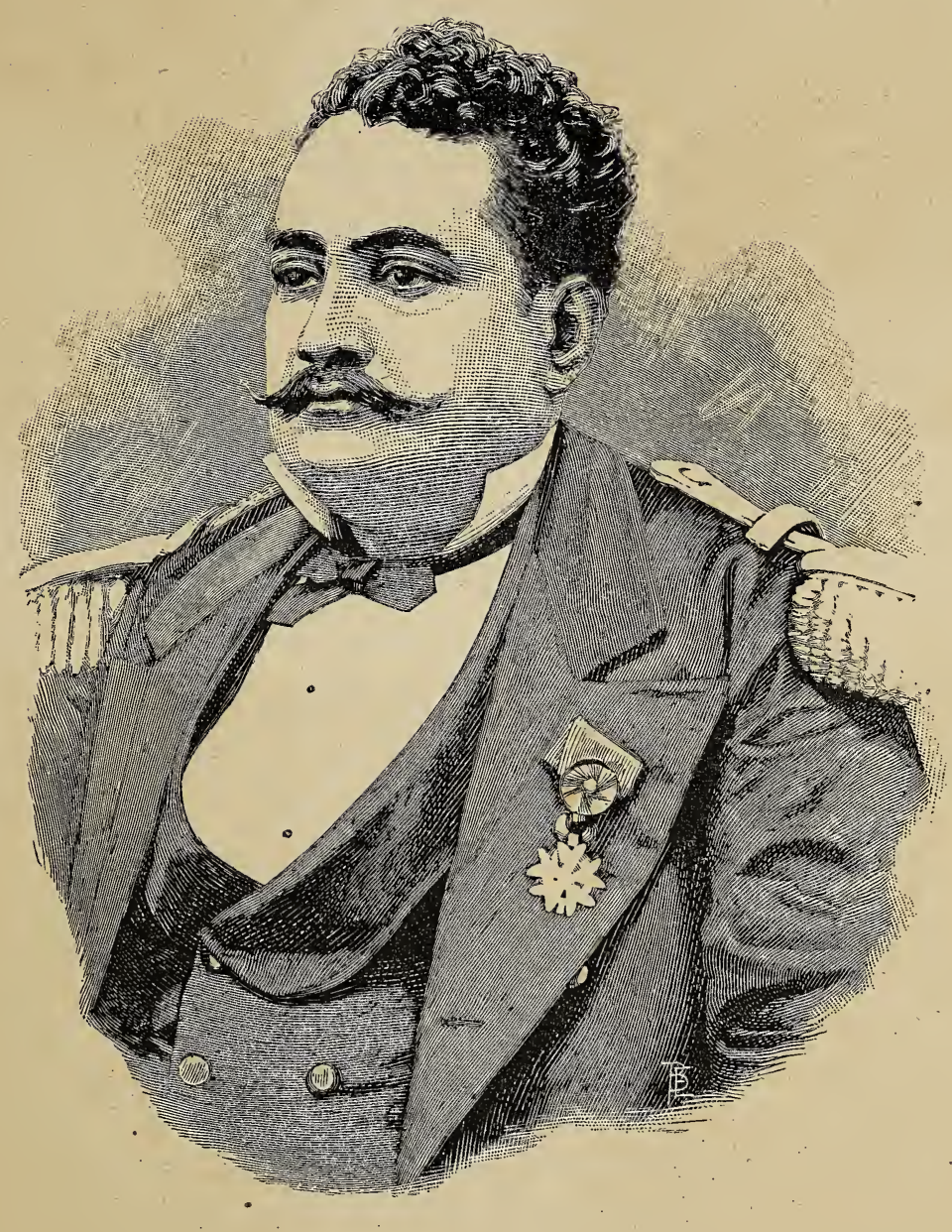

Pomarè V, Re di Tahiti. 
Fac-simile dell'autografo di Re Pomarè V.

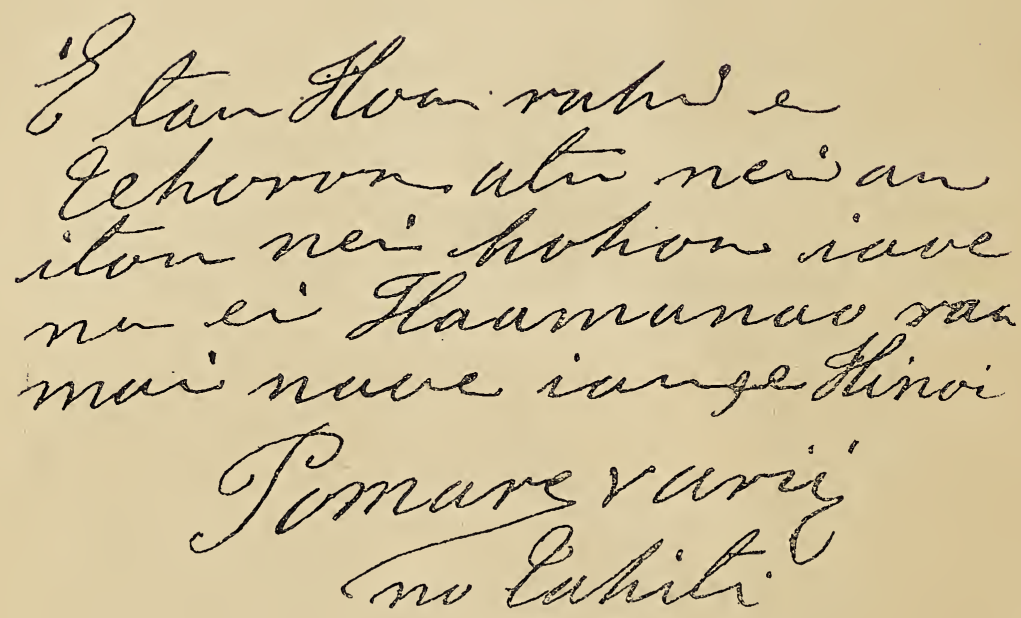

(Traduzione).

L'offerta mia al mio grande amico non soltanto consiste in questo ritratto, ma altresi nel ricordo, che serberò gradito, della sua nave, la quale si prepara a partire. 


\section{TAHITI}





\section{CAPITOLO I.}

REMTNISCENZZE DI TAHITI

I.

Porto di Papeete - Arrivo.

LA notte ci aveva sorpresi nelle vicinanze di Punta Venere dell'isola Tahiti; numerose luci segnavano la giacitura del porto di Papeete, e si andava in cerca dei segnali di approdo. La luna rischiarava l'orizzonte, sicchè ogni oggetto, non lontano, poteva essere distinto; ad un tratto, scorgiamo un'imbarcazione, che muove verso noi: è il Pratico. Arrestiamo la macchina; e poi si riprende la corsa per ancorare.

Papeete, cuore e mente degli Stabilimenti francesi dell'Oceania, festeggia da ieri l'anniversario della Repubblica, dell'ultima, s'intende, e la festa fa dimenticare agli artiglieri, a cui l'Amministrazione locale affida l'accensione dei due fanali rossi di allineamento dell'entrata, che vi è al largo una nave ansiosa di vederli. Il Pratico è abile, quindi procedendo a rilento s'imbocca l'angusto canale di accesso. I due fanali sono accesi, e si penetra nel porto passando presso alla corazzata francese Montcalm dell'Ammiraglio Landolf, che è illuminata a gala. 
Alle 7 pomeridiane, del 14 luglio, si dà fondo, accendendo fuochi Coston all'estremità dei pennoni ed intuonando colla fanfara la Marsigliese. Grida di gioia dalla spiaggia, dalle navi: la Montcalm suona la marcia reale.

Giunge a bordo l'Aiutante di campo del Governatore per invitare lo Stato Maggiore al ballo ufficiale, che avrà principio alle 9. Il cortese invito è accettato, tuttochè la stanchezza del viaggio consigli invece il riposo.

Lo sbarcatoio è buio; si mette piede a terra e si va tentoni, ma tosto vengono ufficiali di marina francesi, che ci tolgono d'impiccio indicandoci il cammino.

Siamo dinanzi ad un'estesa piazza, la piazza del Governo, fiancheggiata da viali, segnati da alti e robusti alberi fronzuti. La luce delle innumerevoli lanterne chinesi e delle lampade a petrolio ci abbaglia; traversiamo una folla compatta, variopinta di abiti e di pelle, straordinariamente gaia.

Il Governatore, Capitano di vascello cav. Dorlodot des Essarts, simpatica figura, accoglie con evidente soddisfazione l'ufficialità italiana. Riverenze al Re Pomarè V di Tahiti ed alla Regina Marau, sua consorte; due coronati, che, per tagliar corto con le difficoltà domestiche e le preoccupazioni dell'avvenire, sono diventati dal 1880 in poi i pensionati di una Repubblica.

Quanta gente! Quanti costumi! e come sono belli questi e com’è attraente quella! Sogniamo?! Chi vagheggiò mai la traduzione al vero di ampissimo palcoscenico, popolato di creature umane dalla fantasia gentile e feconda e dal sentimento amoroso di sapiente coreografo, avrebbe dovuto, in quella notte, porsi nei panni nostri, e toccare e credere.

Alle 2 del mattino si balla ancora, per le vie, e si canta e si ride e si giuoca a gatta cieca. I maturi d'anni scomparvero, ma è rimasta la gioventú, resistente cotanto alle fatiche inscienti del piacere. 
II.

\section{Le feste della Repubblica.}

Questa razza polinesiaca, dalle forme atletiche, dal sembiante avvenente se non bello, dall'incesso maestoso, sembra personificare le massime della scuola di Aristippo: obbiettivo della vita, il piacere; movente delle azioni umane, l'interesse.

E la Francia, che in quarant'anni di occupazione poco potè nell'elevare il livello morale del popolo a lei soggetto, ne seconda però la decisa tendenza alla vita giocosa e facile. Ma costa danaro il far divertire, a suon di legge, diecimila grandi fanciulli; e l'erario francese, geloso a buon diritto dei propri bilanci, lascia ai suoi amministrati bronzini il pensiero di pagarsi le feste, che favorisce, con quello di provvedere ad altri bisogni.

Nella Polinesia francese, le entrate, mercè il regime delle esazioni fluttuanti, dure e protezioniste, debbono equilibrarsi con le uscite, fra le quali figurano i divertimenti pubblici. Questi, per semplificare le cose, vengono condensati nella baldoria di tre a quattro giorni, intitolati Anniversario della Repubblica francese. Accorrono a Tahiti, in tale circostanza, dietro formale appello regolato da norme chiaramente definite, dagli arcipelaghi occupati o protetti dalla Francia, centinaia e centinaia d'indigeni, preceduti e guidati dai rispettivi capi, gonfaloni e tamburi, tutti con gl'indumenti, a colori vivaci, delle occasioni solenni e con il distintivo del proprio distretto, villaggio o tribú.

Tahiti, in quei giorni, assume l'aspetto di un'immensa arena di giocolieri e baccanti, che un pudore innato trattiene sui margini dell'eccesso, e disciplina, nella misura dei piaceri onesti, semprechè gl'istinti brutali non s'impongano. Inenarrabile allora 
lo agitarsi disordinato dei tappeti umani, che al cadere del sole cuoprono strade, viali, piazze, marina: tappeti tessuti di vestali, che disertarono sempre il fuoco sacro, e di gladiatori incruenti. Ha troppa luce il giorno, perchè risaltino i contorni della scena magica; la notte sola dà le immagini fantastiche.

Il domani del ballo ufficiale era domenica, e nell'universale allegria traspariva alcunchè di compassato.

I buoni missionari inglesi, che dai tempi dell'illustre Cook si stanno struggendo per ispiegare la Bibbia a chi, abitante di banchi di corallo, non concepirá mai il paese di Canaan, a furia di torturare il cervello indigeno, riescirono a fare intendere a pochi, che le espansioni del piacere conviene trattenerle almeno il giorno in cui Iddio si riposò.

Il culto protestante, attualmente, predomina nella Polinesia, ma non è improbabile che il culto cattolico, meno rigido e piu suscettibile di colpire le immaginazioni, a lungo andare, prevalga, rispetto all'esteriorità. Apparenza e non sostanza; religione vera, forse mai!

L'allegria domenicale, dicemmo, era compassata, ma era sempre allegria: le corone olezzanti cingevano le teste degli amabili canachi, disseminati ovunque; e i venditori ambulanti di gingilli, punti di attrazione dei curiosi, erano schierati sulla piazza del Governo, in bell'ordine, con un lusso di candele degno dei più miracolosi altarini, ai lati del chiosco, in cui suona la banda ufficiale i giorni festivi.

Dei due viali che fiancheggiano la detta piazza, quello che conduce al palazzo del Governatore, ed è riservato alla classe più distinta, la sera di domenica era silenzioso; l'altro invece, che lambe lo steccato del parco reale, presentava lo spettacolo di vorticose corse d'Europei, seguaci assai piu delle consuetudini indigene che osservatori scrupolosi dei precetti del rito protestante.

Nelle prime ore di lunedi, 16, vi fu risveglio dell'usuale chiassosa gaiezza. Il tamburo, le grida preconizzavano di buon'ora gli himenee di addio al Governatore ed alla Maestá di Pomarè V. 
Ogni distretto ha i suoi canti locali, che chiama himenee e che predilige, poichè sono la base dei suoi passatempi, la genesi delle sue tradizioni, canti privi di melodia ma non disarmoniosi.

A sera, i pressi dell'abitazione reale e del palazzo del Governo sono stipati di gente allegrissima. I rappresentanti dei distretti sfilano nella strada maestra, e si recano, gli uni nel parco del Re, gli altri nel giardino del Governatore.

Si odono già gli himenee, ma vengono in breve a soffocarne le ritmiche note gli squilli della fanfara della Caracciolo, che ha accettato gli onori del chiosco governativo. Itaria! Itaria! è voce che si propaga celeremente e che ripetono a squarciagola vispe e folleggianti fanciulle. Altrove, fuori dei recinti ufficiali, si balla la upaupde, la ginnastica della lascivia.

A mezzanotte, la folla si dirada, e la solitudine si appresta a padroneggiare il campo ; rimarranno però imperterriti, sulla breccia, e vi staranno finchè spunti il giorno, gli instancabili cantori degli himenee del Re!

Siamo al martedì, 17, e la Regina di Raiatea, isola del Protettorato, parte per rimpatriare sull'avviso francese Limier, comandato da un fior di gentiluomo: il Capitano di fregata C..... Le fanno corteggio i suoi sudditi, reduci dalle feste, i quali terranno in non cale la nitidezza del ponte levigato della nave. C..... non perdonerá mai ai devoti di una maestá l'avere macolato quel ponte coll'olio di cocco, e diverrà, per reazione, più repubblicano di prima.

Presso il Governatore si cantano gli himenee finali. Che polmoni! Che serietà! e che stupende corone depongono le canache cantatrici sul seggio del Governatore!

I divertimenti sono terminati e Tahiti ricade nella mollezza del suo clima.

Le feste di cui fummo spettatori erano un sogno, una visione? Ecco: abbiamo veduto attoniti un popolo delirante, la cui mitezza di costumi sembra accennare a remota civiltà, ma che, esausto di forze, deficiente di tempra, va portando fiori sulla sua tomba. 
III.

\section{Visita della Regina alla "Caracciolo و"}

Joanna-Marau-Taaroa-Tepau-Pomarè è nata il 24 aprile 1860, dall' Inglese Salomone e da un'indigena di Tahiti.

Ha il portamento di una regina, al pari di tutte le figlie dell'Oceania, gli occhi neri, lo sguardo dolce, la statura anzi che no alta, le forme giuste. Naso, bocca e colore rivelano l'incrociata origine.

Parla bene il francese e meglio l'inglese. Vive modestamente in una casa canaca, foggiata alla europea, che sita a mare della strada maestra, fa fronte all'abitazione dell'augusto Consorte.

La defunta Regina Pomarè, i cui sentimenti di affetto ondeggiarono, in ogni tempo, tra i freddi puritani Sassoni, che tentarono correggere l'esuberante amore di lei per i piaceri mondani, ed i galanti Franchi, che proponevano per quell'amore accordi col cielo, perduta l'adorata nipotina, la futura erede del trono, divenuto maniaco il primogenito, pensò, un giorno, di dare in moglie al secondo figlio una Rachele, pegno di pace per la sua coscienza tribolata. La Rachele fu Marau, ma una funesta tempesta non tardó ad imperversare, e la nave coniugale naufragò irreparabilmente.

Ciò detto rechiamoci dal Governatore Des Essarts. Egli ama i suoi ospiti italiani e non trascura modo nè occasione per rendere loro gradito il soggiorno di Tahiti: pranzi frequenti, ricevimenti il giovedi.

La Caracciolo ricambia del suo meglio le delicate premure dell'anfitrione, improvvisando serenate, accademie di canto e di 


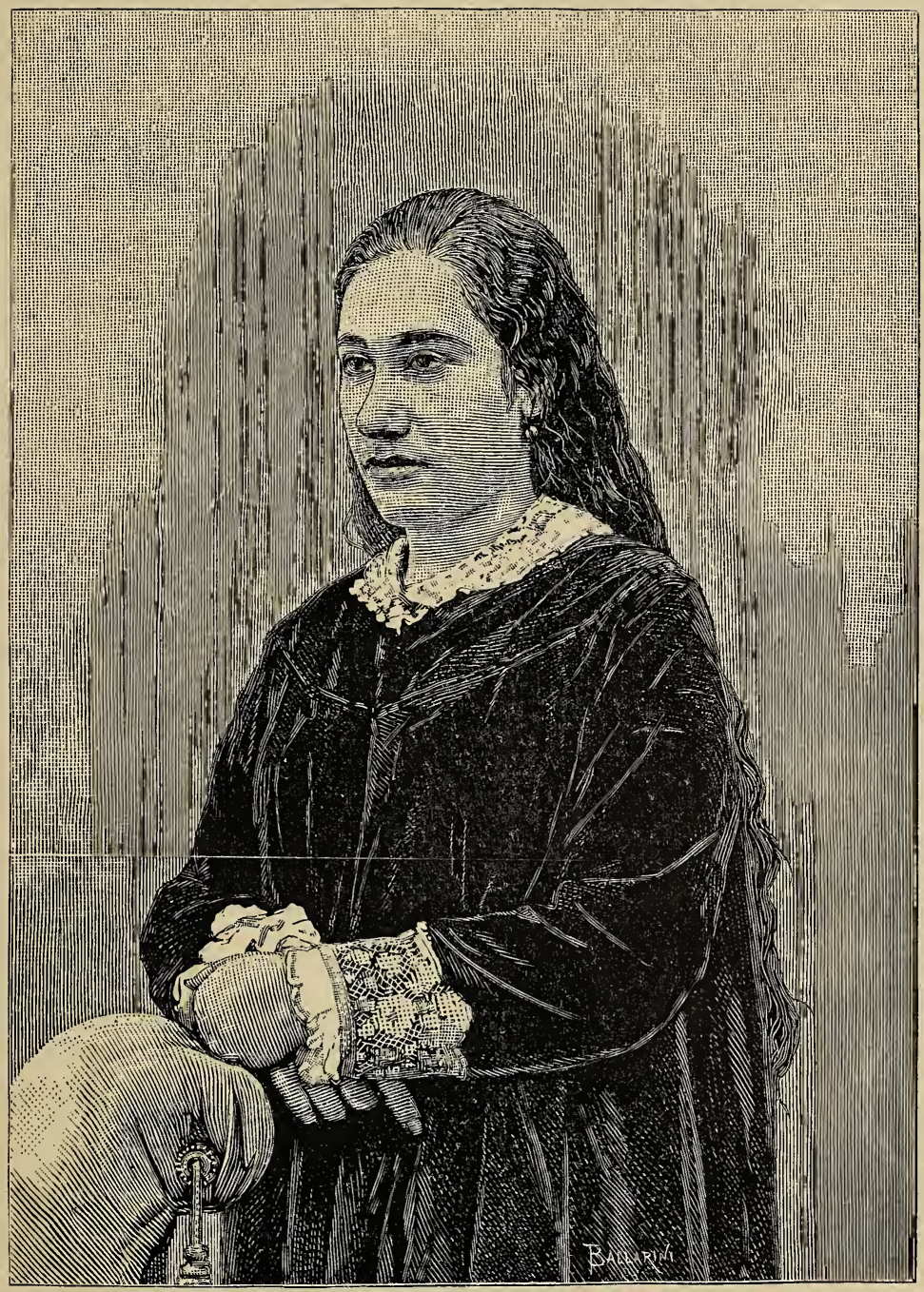

Marau Pomarè. 

burattini. Sì, di burattini, dacchè la Società drammatica di bordo, 1 la quale non potrebbe sempre improvvisare rappresentazioni, conta fra i suoi membri un valente direttore di fantocci di cartone, il Capo cannoniere Quarto, che pregato, non esita mai a dar saggio, in pubblico, dell'abilità della sua automatica compagnia.

I burattini ballavano negli appartamenti del Governatore, in presenza della Corte, di vari funzionari della colonia e di molti indigeni, quando la Regina così interpellò il Comandante della Caracciolo:

- So che al vostro bordo vi sono collezioni di serpi, di leoni, di uccelli e di molti altri oggetti rari: vorrei vederle, perchè sono cose nuove per noi, che viviamo all'oscuro di tutto.

- Agli ordini di V. M. - rispose il Comandante.

Come si riceverá la Regina?

Tahiti fece getto nel 1880 della sua autonomia, ed il Re Pomarè V rinunziò al suo regno, non già alle prerogative sovrane: la lista civile e gli onori del rango per sè e la famiglia. Quindi S. M. Marau, tranne il caso di volontá esplicitamente espressa, di visitare la nostra nave in forma privata, sarà ricevuta a bordo a norma dei regolamenti in vigore. Ma trovansi in porto navi da guerra, ed i segni esteriori di riverenza, da parte nostra, verso Sovrani pupilli della Francia, toccano pure da vicino il Governo francese.

Pertanto, potrebbe la Caracciolo solennizzare, isolatamente, il passaggio della Regina nelle acque del porto di Papeete? Cadde la domanda non appena enunciata. L'Ammiraglio francese Landolf manderà una lancia per prendere la Regina, e nel momento che essa s'imbarcherà, la corazzata Montcalm farà una salve di 21 tiro; la Caracciolo alzerà la piccola gala, mentre la Regina salirà a bordo, ed a sua volta saluterá con 21 colpo S. M., allorchè essa scenderà a terra.

1 Compongono questa Società gli ottimi sott'ufficiali. 
Sabato, 21, alle 2 pomeridiane precise, tuonava il cannone del Montcalm, salutando i colori dei Pomarè. La lancia reale approda alla scala della Caracciolo e sale la Regina col suo corteggio, cosi composto: due Principesse, la cognata e la sorella; un Principe, il fratello; quattro Dame di onore; un Cavaliere ed una Ancella, diavoletto insofferente d'etichetta.

S. M. porta una lunga tunica di raso blù oltremare cupo; cappello di paglia ornato di penne di struzzo; collana d'oro e medaglione con le cifre reali in diamanti. La Principessa sorella, sedicenne, è bianca, veste color rosa, avviluppata in uno scialle, chè deve combattere un raffreddore guadagnato nel famoso anniversario; l'altra Principessa, donna di una certa età e di carni scure, veste una tunica nera. Il Principe, alto e tarchiato quanto un corazziere italiano, bruno e coi baffi, è in frac, che porta con disinvoltura. Il Cavaliere, bianchissimo di pelle, biondo, dagli occhi azzurri, si muove anch'esso dentro un frac. Le Dame di onore se non eclissano per eleganza la loro Sovrana, destano peraltro l'ammirazione dell'equipaggio per la freschezza dei loro abbigliamenti.

Ci troviamo con una Regina tahitiana, che non è ignara del cerimoniale delle Corti europee, e l'osserva appuntino; interroga con affabilità dignnitosa, ed esterna desiderî con la sicurezza che verranno interpretati quali ordini.

A poppa, sul casseretto, era stato improvvisato con bandiere, palme, rami d'alberi, fiori ed armi, un padiglione intorno al quale, sopra gradinate addobbate, si collocarono artisticamente le nostre collezioni.

Due puma innocui, leoni americani, in positura di guardiani, eccitano per i primi la curiosità femminile; vengono poi le serpi, in alcool, e gli uccelli imbalsamati, e i minerali e gli huacos peruviani; ma sono gli uccelli che la Regina e le sue dame osservano piu attentamente; chi sa? rammentando forse le leggende narrate dalle madri, in cui gli animali alati rappresentavano la parte di spiriti erranti.

- E le mummie - domanda S. M. - dove sono? 
- Maestà - si risponde - riposano placidamente nelle loro nicchie, nascoste alla vista dei paurosi.

- Ma io non ho paura - soggiunge la Regina - ed un coro di vocine risolute ripete: - Non abbiamo paura.

Nella sala da pranzo del Comandante, trasformata tosto in un camposanto, si espongono teschi e cadaveri umani, stupendamente conservati. Due dame piangono; le altre, al contrario, fanno pompa di una certa dimestichezza con questi antichi avanzi umani.

Si ritorna sul casseretto, sul quale è preparata una piccola merenda che chiude il programma della visita augusta.

Alle 4 pomeridiane la lancia d'onore francese è presso la scala. La Regina fa la piu graziosa delle sue riverenze, ringrazia, si accommiata e scende. Principe e Principesse si dirigono verso il barcarizzo di dritta, dando uno sguardo ancora al motto di occasione, scritto sul coronamento del casseretto: Ja ora na Marau. Ti saluto Regina Marau.

La lancia reale è giả lontana; i delicati timpani saranno meno offesi, e però i cannoni della Caracciolo eseguiscono la salve convenuta di 21 tiro.

IV.

Una colazione canaca.

La novità per noi di una colazione indigena, ci aveva resi puntualissimi all' invito del signor Snow, sicchè potemmo assistere alla levata dall'umu ${ }^{1}$ del bel maiale, che doveva rappre-

1 È il forno canaco, di forma emisferica, che si scalda a gran fuoco assieme con molte pietre, ed in cui si depongono poi le vivande, avvolte in foglie di tarò, per farle cuocere, ricuoprendole con terra e pietre roventi. 
sentare il solito piatto forte, indispensabile in ogni pasto polinesiano.

Una tenda di cuopriletti (tifaifai) dai colori smaglianti e dagli arabeschi strani, adornata di fronde di cocco e di grandi felci, formava la sala del convito.

Serviva da tavola il suolo coperto fittamente di foglie; le anfore erano noci di cocco contenenti il latte del frutto, e le stoviglie erano foglie, nient'altro che foglie di purau.

Ci sdraiammo su stuoie, alla guisa dei Romani nel triclinio, e fummo inghirlandati. Ma non durò l'illusione di crederci trasportati in una delle antiche ville del Lazio, famose per le feste luculliane. Il pesce crudo, l'amido bollito, i fei, i tuberi d'ogni specie; taró, ignami, patate dolci, ci tenevano stretti alla realtà, che apparve singolarmente cruda, quando i famigli, prendendo il nostro posto, si diedero a liquidare gli avanzi abbandonati della nostra colazione.

Un pezzo d'uomo alto e ben pasciuto, un eroe dell'Iliade e dotato di un appetito non meno omerico, aprì questa seconda seduta bucolica, mettendosi all'opera con la voracitá di una belva, e mostrando i denti bianchissimi, forti e saldamente piantati, $\epsilon$ gli occhi sfavillanti di selvaggio piacere.

Allorchè gli Europei scopersero le Isole della Societá, non vi trovarono traccia di cannibalismo, quantunque i sacrifizi umani fossero di uso frequente; nonostante, anche oggidì in alcune isole della Polinesia, popolate dalla stessa razza, l'antropofagia è in onore. Quell' uomo dall' invidiabile appetito ci faceva pensare appunto ai cannibali; e chi sa se egli non avrebbe divorato con uguale gusto, esclusa ogni molestia legale, un po' di carne umana, di carne dell'uomo bianco in ispecie, che i conoscitori dicono abbia molti punti di somiglianza, per sapore, con la carne di maiale. ${ }^{1}$

${ }^{1}$ Così affermava a Suva (Fiji) persona degna di fede, la quale, in intima relazione coi vecchi Capi delle isole, raffinati antropofagi, aveva appreso dai medesimi che tale era il sapore della carne dell' uomo bianco. 
Ma lasciamo le riflessioni importune e diamo, in omaggio alla munificenza dol padrone di casa, la distinta del fiero pasto:

Eia ota - pesce crudo con salsa di cocco, limone ed acqua. di mare;

Oura pape - gamberelli in forno canaco;

Paupa - granchi idem;

Eia tarea - pesce cotto in salsa di pimento;

Pota - legumi, tarò e sugo in forno canaco;

Eia Puohu - pesce cotto con salsa verde;

Uru o maiorè, feitarò e hui maara - frutto dell'albero del pane, banane selvatiche, legumi, ecc. (succedanei del pane), in forno canaco;

Poe pia - amido col latte di cocco;

Poe tarò - tarò col latte di cocco;

$\mathrm{V} i$ - mele citerne (specie di mango);

Anoni - arancie;

Papa haari - cocco.

La distinta della colazione (fattura europea) portava l'iscrizione: Amura maa $i$ horo a hia na te Tomaua no te Pahi Itaria, che significa: Colazione data al Comandante della nave italiana.

I canachi non sono ricchi di stoviglie, nè di altri utensili da mensa o da cucina, ma la più perfetta pulitezza presiede sempre alla preparazione delle vivande ed al loro consumo. Nessun oggetto è mai preso colle mani nude dai preparatori, i quali si servono costantémente di foglie per toccare i cibi. I consumatori adoperano bravamente le dita, per mangiare, ma hanno cura d'immergerle ogni tanto in una coppa ripiena d'acqua limpidissima. 
V.

\section{Gita all' isola di Moorea.}

Il Governatore Des Essarts ci consigliava di non uscire dalle acque di Tahiti senza avere visitato Moorea, l' isola gemella, che ha l'occhio ciclopico. ${ }^{1} \mathrm{E}$ sulla via che deve condurci alle isole Figi, seconda sosta dell' Oceania, e possiede due baie degne dell'attenzione del marino.

Una di esse, quella di Papeetoai, attorniata di campi feraci, mostra, nello sfondo, una ragguardevole fattoria produttrice di cotone, che fu fondata da un italiano, il signor Micheli, e ch'egli vendè non ha guari, per 200,000 lire, al tedesco signor Godoj.

Moorea è più pittoresca, più ridente di Tahiti; colá le disordinate eruzioni vulcaniche non hanno piu l'antica nuditá; la folta vegetazione si stende dalla cresta alle falde dei monti. La schiera infinita dei pazienti architetti del mare vi spiega una straordinaria attivitá, e noi potemmo, con la lama del coltello, staccare dall'orlo esterno delle profonde muraglie coralline, saggi morbidi di cui ogni molecola è animata.

Accettato il consiglio, partiamo la mattina, del 28 luglio, da Papeete, con la scorta del Tenente del porto signor Garnier, abile piloto locale. Abbiamo di passaggio il Residente governativo di Moorea, l'ottimo signor Migard-Savin, Tenente di fanteria marina: Giudice, Questore, Tesoriere, Sindaco e Prefetto dell' isola; ed inoltre alcuni indigeni, fra i quali la signora Reo, buona canaca puro sangue, sebbene di color chiaro, che, legittima erede del

${ }^{1}$ Uno dei monti di Moorea, che sovrastanno ad Afareihaitu, fa vedere dal mare, sulla sua cresta, un foro anulare che trapassa da banda a banda. 
Governo di Afareihaitu a Moorea, per abbandonarsi liberamente ai suoi gusti di farfalla, lascia al padre, Hapoto, l'autorità ed i fastidi del comando. Accompagna la signora Reo la signora Taponi Snow, nata Gilson, semi-inglese e semi-indigena, donna di molto garbo, influente a Papeete ed altrove negli arcipelaghi francesi, ed a cui i Governatori di Tahiti ricorrono da vari anni, affinchè l'ospitalità da loro offerta agli stranieri non abbia, in mezzo alle costumanze europee, da perdere la sua bella impronta canaca. La signora Taponi Snow coadiuverá il Residente Migard-Savin nel procacciare distrazioni all'equipaggio della Caracciolo durante il suo soggiorno a Moorea.

Giungiamo alle 11 antimeridiane nella baia di Papeetoai, c scendiamo subito a terra per vedere, in fretta, il distretto di Papeetoai, sede del Residente e di un gendarme, e la fattoria Godoj. Splendida baia, amenissimo distretto, estesa e bene ordinata fattoria.

Ripartiamo alle 2 pomeridiane ed ancoriamo alle 5 pomeridiane in rada di Vaierẻ, distretto di Teaharoa.

Nessuno ci aspetta a Teaharoa, ma ad Afareihaitu, ove Hapoto ed i suoi amministrati sanno che una Pahis Itaria - una gran canoa italiana - deve fare la sua apparizione nelle acque di Moorea, vi è movimento insolito e si fanno preparativi di sontuoso ricevimento.

Teaharoa, ad onta dell'eccellente porto di Vaieré, è distretto troppo sparso per poter fare convenientemente gli onori di casa, mentre quello di Afareihaitu dispone di un ponte sbarcatoio per lance, di un'elegante Chefferie - casa del Governo - di un tempio protestante, di una casa per le riunioni pubbliche - Fare hau di passeggiate e di molte altre belle cose.

Dobbiamo sbrigarci; la notte si avvicina e la navigazione fra Vaierẻ ed Afareihaitu - tre miglia circa - sia pure per le imbarcazioni leggiere, è problema arduo, che non si risolve con l'oscurità, se si vogliono evitare le insidiose escrescenze del fondo.

Un'ora e mezzo di cammino, in parte a ghirigori, onde poter 
salutare il bravo Hapoto, che tutto vestito di nero, con l'abito a coda di rondine è venuto al nostro incontro sul ponte, seguito dal Consiglio del distretto e da molti uomini, donne e fanciulli in abiti di parata.

Accoglienza festosa, dopo cui è assegnata a ciascuno di noi, nella casa del Governo, una camera ben pulita, come tutto ciò che è canaco. Alle 8 di sera, pranzo di gala, un'amuraamaa, che il distretto dà alla Caracciolo nella casa delle riunioni pubbliche. Una piattaforma difesa da esile cancello separa l'ufficialità italiana ed i personaggi indigeni dal pubblico, che annovera una trentina dei nostri marinai; ma si è tutti seduti a terra, con il capo cinto di corone di palma, usando delle stesse stoviglie - foglie, cioè, di purau, - mangiando delle stesse vivande e bevendo acqua di cocco.

La serata è quasi a beneficio esclusivo dei nostri marinai, i quali, affratellatisi presto con gli indigeni, gareggiano con essi nel far chiasso e nel divorare carne di maiale, fei, ignami. Ma le innocenti libazioni di acqua di cocco non possono generare agitazioni soverchiamente rumorose; quindi, a pranzo finito, il dileguarsi progressivo dei commensali si opera serenamente, dando luogo, verso le 11 pomeridiane, al riposo generale.

Il mattino si va al Marae di Afareihaitu. Il marae era il tempio, allo scoperto, della religione tahitiana; si componeva di un recinto di forma pressochè rettangolare e di un altare a guisa di rialzo, che occupava il centro tra i due grandi lati. Di quello di Afareihaitu rimangono scarse vestigia: un muro assai bene conservato, che porge a mare; un'elevazione di pietre al centro.

Si odono i rintocchi del tempio: è giorno di domenica, e la popolazione accorre ad assistere all'ufficio divino che reciterá, con compunzione, il savio Vaitoaré, il pastore canaco di Afareihaitu.

Spronati dal buon esempio, ci avviamo anche noi al tempio. Siamo nella casa del Signore. In fondo, due pulpiti, l'uno sovrapposto all'altro; da' due lati varie file di panche; tre passaggi: uno al centro e due di fianco; la campana sul suolo, vicino alla 


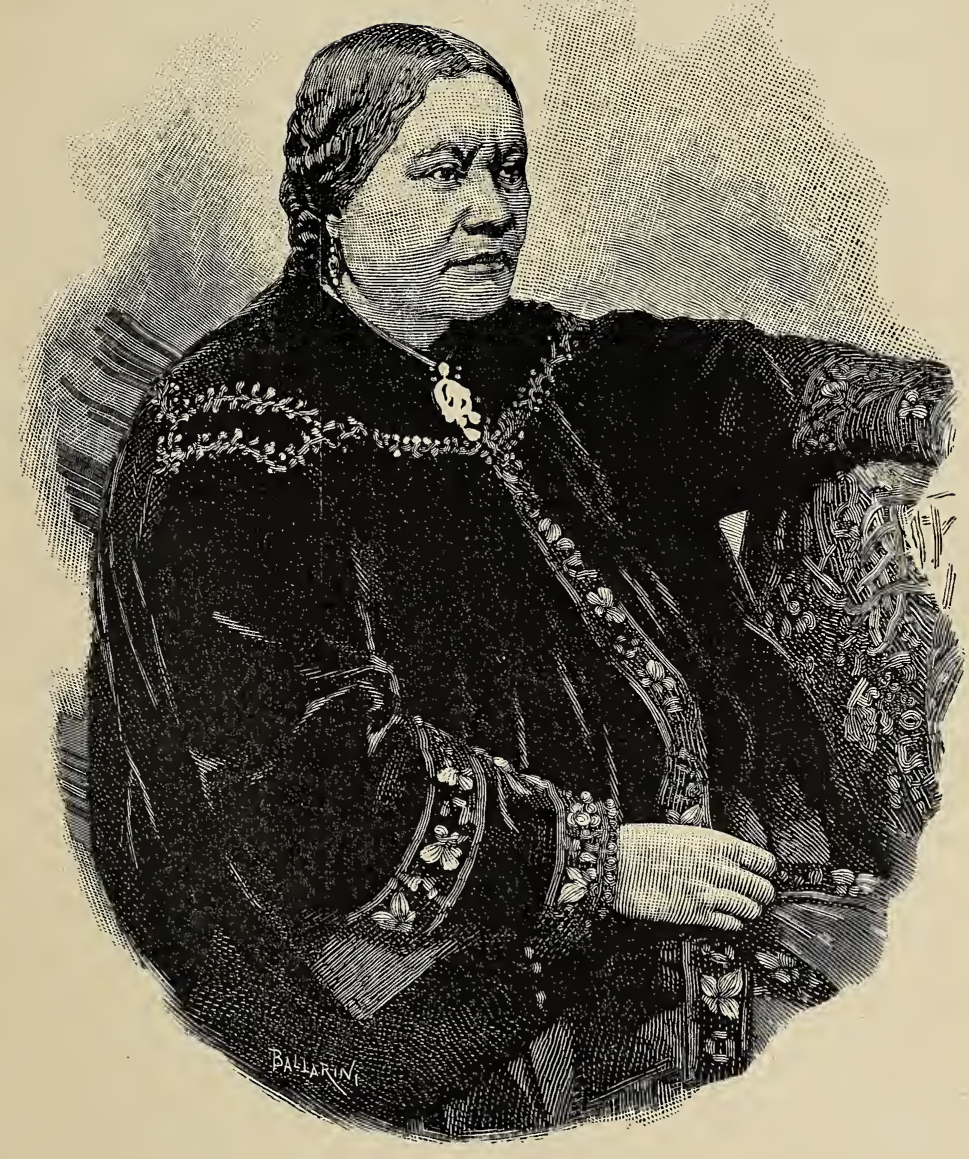

Regina Pomarè IV. 

porta. Pavimento di tavole nitidissimo, soffitto di rami di purau rivestito di pandano, pareti di rami levigati e aderenti pure di purau.

Vaitoaré, in corretto costume di ministro europeo, prende posto sul più basso pulpito ed intuona un inno che è cantato da donne e fanciulli. Dopo l'inno, la preghiera; si era seduti, ed ora si dovrebbe stare ginocchioni; ma i religiosi canachi hanno ideato una posizione meno incomoda: fanno un quarto di giro sulla panca e pregano col viso rivolto alla porta.

La preghiera di oggi è preghiera eccezionale, di occasione, dovuta all'improvvisazione ed all'eloquenza di Vaitoarè, ciò che d'altronde non deve sorprendere, quando si sappia che l'eloquenza è dote naturale dei Polinesiani, e che a pochi, o meglio a nessun indigeno di qualitá, fa difetto la vena oratoria.

« Signore - dice il Ministro - esaudisci le nostre preci, togli dall'errore i nostri cuori, tu che puoi farlo, imperocchè sei potente, e della tua potenza ci dai prove quotidiane. Oggi ancora questi stranieri, questi ufficiali europei investiti di gloria e di autorità temporali, questi uomini che sono in mezzo a noi per abitarvi e mangiare, nel presente giorno solenne, quantunque venuti da tutti i punti del globo, non sono forse qui dinanzi a te, ascoltando le tue lodi, che noi ti cantiamo, le preci che noi t'indirizziamo, ed eglino non cantano e non pregano pure dal fondo del loro cuore, se non con la bocca?

« Tu ci dai novella prova e splendida della tua grandezza, e per essi ti indirizziamo la seguente supplica:

« Concedi a noi di trattarli bene, corporalmente, durante il loro soggiorno fra noi, da' loro la pace dello spirito, e quando lascieranno queste rive, diretti ad altri paesi, segui la loro nave co' tuoi occhi, proteggila contro gli scogli, concedi loro di rientrare in patria, per portare la gioia nel seno delle loro famiglie. «Amen », rispose l'uditorio.

L'ufficio divino compiuto, usciamo con grli amici canachi, i quali ritornano alle loro case o capanne in atteggiamento

Viaggio di circumnavigazione. Vol. II. 
grave, con la Bibbia sotto il braccio, o portata a mano entro finissimo cestino di pandano. Quella Bibbia, stampata a Londra ed in tahitiano, affermano gl'intenditori, è un gioiello di letteratura indigena; gioiello destinato, in un avvenire prossimo, ad essere l'unico documento atto a ricordare i pregi della originaria lingua tahitiana, che rapidamente si estingue con lo innesto dei vocaboli stranieri.

Vaitoarẻ, pastore tollerante, permette a' fedeli di godersi nelle ore pomeridiane la fanfara della Caracciolo, recatasi ad Afareihaitu; e si unisce con loro per assistere, a notte fatta, ad una rappresentazione dei nostri fantocci di cartone, nella casa delle riunioni pubbliche.

Il lunedi, fin dalle prime ore del giorno, gli abitanti di Afareihaitu e di Teaharea si sono riversati sulla nostra nave, invadendo coperta e corridoio, appartamenti, ogni piú recondito nascondiglio. Prepotente curiosità spinge da un punto all'altro della Caracciolo quei buoni indigeni, i quali incessantemente guardano, toccano, ammirano, sorpresi dell'ampiezza dello spazio e degli oggetti che contiene. Si muovono a gruppi, con il saluto ed il sorriso sempre pronti; e giunta l'ora del pranzo dell'equipaggio, fanno buon viso a quelle porzioni del pranzo stesso, che i nostri marinai, con la consueta bonomia, loro distribuiscono.

Tante dimostrazioni di simpatia dagli abitanti di Moorea, prodigateci con spontaneità, vogliono essere apprezzate con una certa evidenza di forme che risponda agli usi del paese. Il Comandante della Caracciolo darà a terra un pranzo di commiato al distretto di Afareihaitu.

Martedi, vigilia della partenza, alle 2 pomeridiane, vengono riuniti dal Residente francese, sulla piazza del Governo, i notabili indigeni, ed in presenza loro portati cinque grassi maiali; il Comandante della Caracciolo prega di farli accettare al distretto, come testimonianza di gratitudine per la splendida ospitalità, e per poter avere un'ultima volta occasione di sedere alla sua tavola. Hurrà degli astanti ed arringa di Hapoto, che gradisce il 
cortese pensiero in nome del distretto e fa augurî di prosperitá all'Italia.

Il cognato di Hapoto, l'anziano della famiglia dei Capi di Afareihaitu, conformandosi ad una vecchia consuetudine canaca, relativa all'ospitalità data, si appropria, nella circostanza, il nome di Taro - Carlo - che è il nome di battesimo del Comandante della Caracciolo, ed impone a questi il suo di Tarioré.

I forni canachi erano stati, immediatamente dopo l'offerta del dono, messi in piena attività, ed alle 8 pomeridiane il pranzo potè essere servito fra gli applausi generali.

Si parla molto, si ride e si canta; alle 11 pomeridiane una upaupd decente, ingentilita per volontaria condiscendenza del distretto alle morali suggestioni del savio Vaitoarẻ, chiude la festa.

Sorge l'alba di martedì $1^{\circ}$ agosto.

Le lance sono al ponte armate; la popolazione è sulla riva; abbracci, strette di mano, e poi si larga e si voga. La brezza per lungo tratto porta ai nostri orecchi l'armoniosa frase tahitiana: Ia ora na Itaria! - Ti salutiamo, Italia! - che maschie voci dagli estremi del ponte ripetono.

VI.

\section{L a $\mathbf{p}$ a u pà.}

Il tahitiano, questo fanciullo eternamente ozioso, beniamino della natura, figlio di un clima dolcissimo, ama i canti e le danze con inusitata passione, sicchè dedica agli uni ed alle altre, nel carnevale della sua vita, lunghe, lunghissime ore.

Gli himenee, che sono i suoi canti, destano meraviglia per la precisione degli accordi, la cadenza perfetta; le upaupdi, che 
sono le sue danze, la destano per le complicate evoluzioni, la esattezza matematica dell' insieme dei movimenti.

La coreografia indigena non è meno originale che svariata; upaupà hura, upaupd mari, mohamoha, otooto, pararaa pehupehu, poopootati, rohi pehe, tiarau, ecc.; upaupd per tutti i gusti, tutti gli umori, tutti i sentimenti, tutti i desiderî. Aggiungasi l'instancabilità degli esecutori.

Nel concetto canaco, ogni circostanza è buona per improvvisare danze; e non v'ha festa indigena privata o pubblica in cui non primeggi la upaupà ed essa non dia l'intonazione all'allegria.

A Moorea, il distretto di Afareihaitu, nel suo programma delle feste, in onore della Caracciolo, non aveva certamente dimenticato la upaup $\dot{a}$; ed il singolare divertimento ci fu dato, di notte, la vigilia della partenza, nel fare hau (casa delle riunioni pubbliche) illuminato da lampioncini giapponesi, addobbato con fronde di palmizi ed animato da uomini e donne inghirlandati e vestiti a festa.

Quella notte noi aspettavamo ansiosi, sdraiati a terra, il principio della upaupà, quando comparve il nucleo dell'orchestra: un tamburo ed un tam-tam, seguito dagl' istrumenti melodiosi: parecchi flauti e pifferi suonati con il naso. Il tamburo era un tronco di cocco vuoto, su cui una pelle di squalo tesa; il tam-tam era un catino di ferro.

Giunse dopo, applaudito da tutti, il corpo di ballo: otto indigeni forti ed agili, guidati dal loro bravo maestro, canaco svelto fra i piu svelti, ed oratore.

Agli applausi succedeva uno speech dal maestro diretto al pubblico, per dare ragione della solennitá e della upaupda, ed allo speech seguiva il segnale della danza.

Le figure e le evoluzioni esordirono con metro moderato, con magistero grave, ma non tardò il mutamento di scena; il crescendo non ebbe più misura e la rapiditá dei movimenti delle membra divenne vertiginosa.

Il pubblico sensibile a tale e tanta frenesia, tratto tratto accom- 
pagnava la danza con canzoni amorose e mandava sordi e prolungati tu-tue, tu-tue - dimenatevi! dondolatevi! Allora il maestro vibrava maggiormente le esclamazioni stentoree di Envoyez les couleurs! Issez le pavillon! ed altre ugualmente comiche, per animare la sua truppa, e qualche donna, esitante dapprima, arrendevole infine alle istanze dei vicini, si recava a prendere parte al ballo, provando così come l'elasticità della persona non è prerogativa soltanto del sesso forte.

Imperiosi tapiti (bis) dell'inebriata assemblea tolsero ogni possibilità di sosta ai nostri ballerini indigeni, per modo che, venuto il momento dello sgombro del fare hau, essi contavano gią due ore circa d'incessante azione coreografica, ma però con l'aspetto di gente fresca e disposta, il caso occorrendo, a ballare un'altra upaupà, fosse magari la upaupà poopootati, la piu lunga a pronunziarsi, e probabilmente la più difficile ad eseguirsi.

VII.

La pesca del tonno.

La pesca del tonno, presso i tahitiani, ha un'impronta cosi originale, da far supporre che sia una creazione indigena, e da giustificare, per lo meno, la descrizione che tenteremo farne.

Premettiamo che il tonno che si pesca in questi paraggi non è mai di grandi dimensioni: da 100 a 150 chilogrammi al più di peso.

Si pesca con canoe gemelle, cioè con due lunghe canoe, di 9 banchi o rematori ognuna, saldamente connesse insieme, ma con un vano in mezzo, per il libero maneggio dei remi dal lato dell'accoppiamento. In questo vano, legata alle canoe, è immersa 
nell'acqua una gran cesta permeabile, che contiene l'esca, per il tonno, consistente in pesciolini viventi.

Dinanzi alla cesta (a pruavia) un traversino di legno, fissato alle due canoe, sostiene una pertica centrale, articolata alla base, che, tirata da poppa mercè due funi, prende a piacimento la posizione orizzontale, ovvero la verticale. All'estremità esterna della pertica, nel punto ove hanno presa stabile le dette funi, trovasi innestata una forcella di legno, a' corni della quale pendono due lenze e rispettivi ami.

A prua di ogni canoa vi ha una piattaforma, su cui va a collocarsi l'uomo destinato ad uccidere il tonno preso all'amo, che corrisponde alla propria canoa.

Questi che abbiamo citati sono gli ordigni della pesca; diciamo ora dell'operazione:

La vigilia della pesca, gli equipaggi delle canoe gemelle si procurano i pesciolini per l'esca e li depongono nella nota cesta. Parte la spedizione all'alba, dirigendo verso la località in cui comunemente si effettua il passaggio dei tonni, e giuntavi si arresta onde scoprire la loro presenza. Scôrti i tonni, i rematori del centro, muniti di cestini, riempiono questi di piccoli pesci, attinti alla grande cesta, e, vibrato un colpo sull'orlo della canoa col cestino stesso per tramortirli, li lanciano al largo a modo di ventaglio. Nel frattempo i rematori poppieri e prodieri vogano indietro, mentre l'uomo dell'estrema prua di ciascuna canoa mette l'esca al suo amo e lo getta in mare.

I tonni si affollano attorno alle canoe e, da ghiotti che sono, voracemente ed alla cieca piombano sull'esca. 亡̀ in questa corsa confusa che cadono vittima degli ami insidiosi.

Con la preda, cessa il getto dell'esca: i rematori poppieri tirano le funi ed inalberano la pertica, quelli del centro vogano in avanti, ed il prodiere salta prontamente sulla piattaforma per afferrare con una mano nelle gargie il tonno, sollevarlo dall'acqua con la pertica, e colpirlo a morte sulla testa con l'altra mano armata di una mazza di legno. Il tonno, svincolato dall'amo, è 
abbandonato in fondo della canoa. Si avanzano allora i rematori a prua, scavalcano un banco, ed il prodiere uccisore del tonno passa a sedersi sull'ultimo banco a poppa.

Ricomincia il giuoco, con l'abbassamento della pertica, e prosegue invariabilmente fino a che, cariche le canoe o stanchi gli uomini, non venga dato dall' anziano, che dirige, il segnale del ritorno.

La piattaforma è posto di onore, e il prodiere, che mancando l'uccisione della preda si sarebbe reso indegno di tale distinzione, troverebbe nell' indignazione de' colleghi pungentissimo castigo. È grazie al mutamento successivo del prodiere, che tutti gli uomini indistintamente dell'equipaggio di ogni canoa, cuoprono, a turno, le varie funzioni inerenti a quel genere di pesca. 



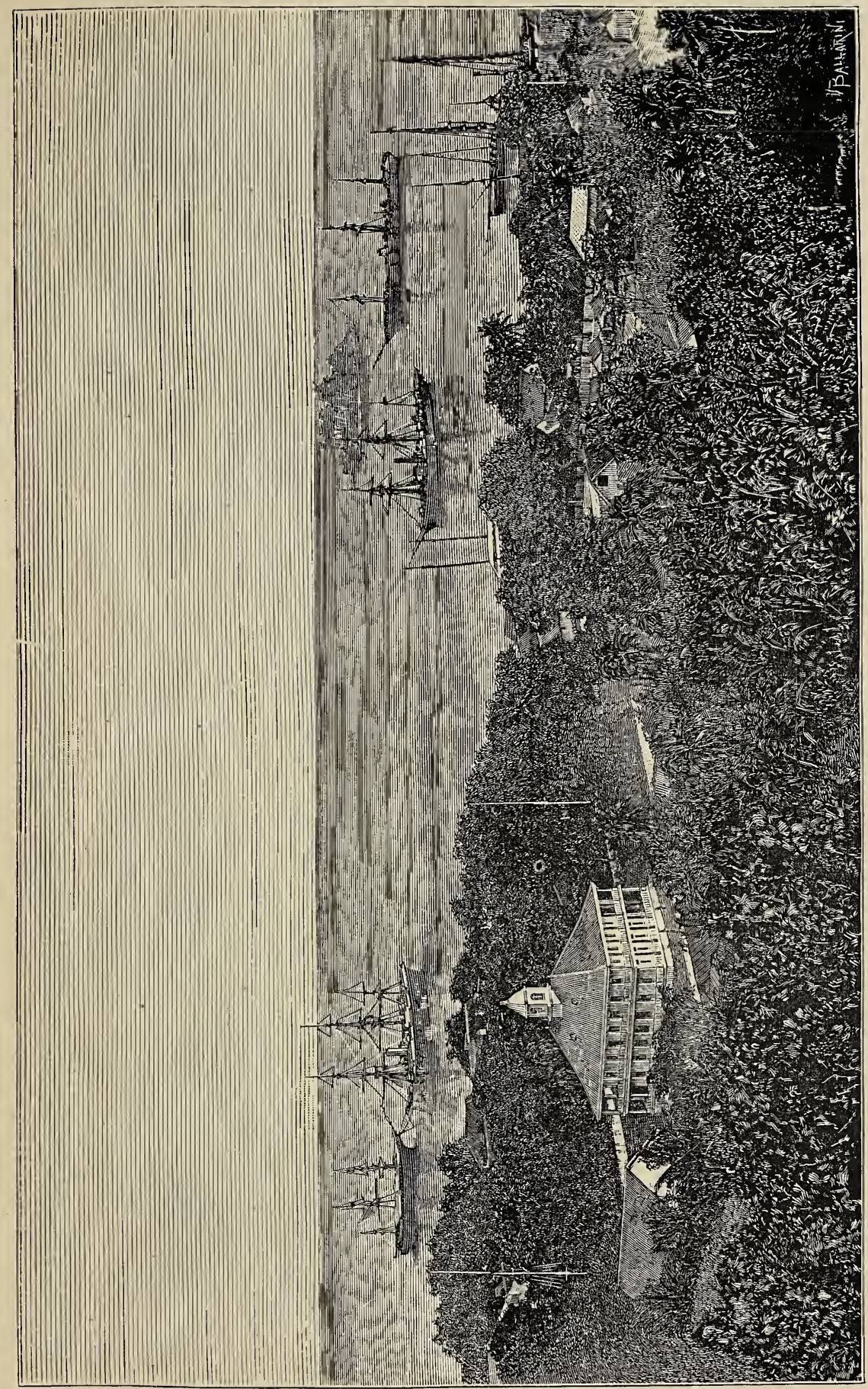

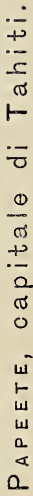





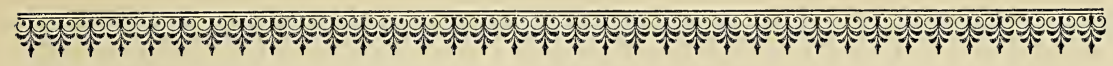

\title{
CAPITOLO II.
}

\author{
CW N NI STORIOI
}

I.

Religione, usi e costumi.

IL Dio Mahoi, l'Essenza divina, pesca la terra con il suo amo, ma Rou, il Dio del vento, spezza Fenna Nui, la Terra Grande, e lascia solo sussistere le Isole.

É la vaga reminiscenza di un gran continente occidentale, da cui emigrò la gente tahitiana, per rifugiarsi sulle isole della Societá, che la tradizione evoca, ovvero è quella di una vasta terra sprofondata nel mare, della quale Tahiti non sarebbe che un avanzo risorto, per spinta plutonica?

Nessuno oserebbe rispondere categoricamente alla domanda. La Polinesia è regione rimasta troppo a lungo sottratta alle inve-

1 Per maggiori particolari consultare: I viaggi di Cook, Bougainville, Wilson, Fitz Roy; Il viaggio alle isole del Grande Oceano, Mourenhout; L'isola di Tahiti, Vincendon Dumoulin; L'annuario di Tahiti del 1863; Polynesian Researches, W. Ellis; Polynesian Mythology, G. Grey; Polynesian Reminiscences, W. J. Pritchard. 
stigazioni minuziose della scienza, per essere intimamente conosciuta, e forse non lo sarà mai, dacchè mancano già, per riedificare il suo passato, molti e valevoli puntelli, che i secoli distrussero, cancellandone ogni impronta.

La lingua, i monumenti, gli usi ed i costumi, coscienziosamente studiati, varranno a gettare, un giorno, alcuni sprazzi di luce sulla storia del Paese polinesiano, cosi strano in tutti i suoi caratteri, mentre forniranno adeguate tinte alla tavolozza di coloro che si accingeranno a dipingere, al vero, il quadro pittoresco.

Noi restiamo nella sfera del narratore, che porge al pubblico notizie raccolte sui luoghi e sui libri e convalidate dall'autoritá delle persone da cui si attinsero.

Le Tongatabu, ${ }^{1}$ isole sacre, isole del popolo piu bello e più forte, si trovano ecclissate, rella vita storica della Polinesia, dalle isole della Societá, da Raiatea, ${ }^{2}$ la culla dei Re e della Religione, e da Tahiti, in particolare, l'Atene di questa Grecia dagli atleti dei muscoli, e non della sapienza. Ma i monumenti bisognerà cercarli, rimontando all' Est di Tahiti due mila miglia, in un' isola meschina, derelitta, nell' isola Pasqua, che ha conservato, da quanto si suppone, statue ed iscrizioni geroglifiche della remota civiltá polinesiana. ${ }^{3}$

Tahiti più ciarliera, più esposta alla curiosità degli investigatori europei, meglio di ogni altra terra è in grado di mostrarci la mitologia di questi popoli, la loro religione ed il loro stato sociale, fino al momento in cui il bianco, coll'urto della volontá prepotente, venne a rovesciare il vecchio edificio etnico dell' Oceania.

Rohu Atua, che è la storia degli Dei tahitiani, o sacra, storia.

${ }^{1}$ Sono le isole degli Amici, situate miglia 1400 a Ponente delle isole della Società.

2 Una delle isole della Società a miglia 120 circa da Tahiti, a Ponente.

${ }^{3}$ Nelle collezioni del bordo conservavansi alcuni geroglifici dell' isola Pasqua, dono del Comandante Gormaz Vidal. 
trasmessa oralmente dall'una all'altra generazione, ha per Giove Taaroa, l'Ente creatore di ogni cosa.

Taaroa creò la Terra e l' Universo, da cui nacque la famiglia degli Dei, che collocó nel Po, regione delle tenebre; e poi diede vita, con le scheggie di un pezzo di aito (legno di ferro) ad una seconda categoria di divinità chiamate a sopraintendere agli affari mondani di ogni natura. Delle deità secondarie la più strana era Hero, il protettore dei ladri.

L'uomo discese da Taaroa e da sua figlia Hina, che attualmente abita nella luna.

Rohu Atua - la storia sacra - dice ancora, che Tutapu e sua moglie abitavano una terra detta Puatiriura, e che non trovando in questa un marito per l'unica figlia Hotuhiva, la posero in un tamburo - Tahi - e l'abbandonarono in mare affidandola alla tutela di Tane, o del Dio o idolo Taputura. I due naviganti approdarono a Manunu, in Huaine, che significa intirizzito. Colà la signorina Hotuhiva prese a marito un Capo chiamato Teaani Maruia; e gli sposi ebbero due figli, Tina ed Hena, che sono considerati come progenitori dei presenti Capi. Tane divenne il Dio tutelare di Huaine.

Il tahitiano credeva all'immortalità dell'anima (varona), la quale, spenta la vita terrestre, tornava alla sua origine nel mentovato Po degli Dei secondari, dopo essere stata assoggettata ad un giudizio divino. La sede dell'anima era il ventre ( $I$ roto $t i$ abou).

Egli avera i suoi Dei lari, specie di genî individuali, che sceglieva, comunemente, fra gli animali, immaginando che, nonostante l'ultima dimora nel Po, essi avrebbero accolto il suo spirito; il che accenna a idee di metamorfosi o metempsicosi.

Fondata ogni sua credenza religiosa sulle piu perverse passioni umane, attribuite agli Dei, e, per conseguenza, sul timore di essere colpito dai loro molteplici effetti; il tahitiano cercava protettori e difensori in ogni dove; ed il culto esterno, il rito, doveano essere, necessariamente, complicati e solenni. 
Il marae, ossia il tempio, era formato da un semplice muricciuolo di pietre coralline, alto da 4 a 6 metri, terminando con una piramide, spesso colossale, 60 metri di altezza con una base di 20 metri di larghezza sopra 300 di lunghezza. Esistevano templi nazionali e templi privati, sì gli uni che gli altri contenenti piccole case destinate a' preti ed alle immagini. Gli altari consistevano in banchi nudi, sui quali si adagiavano le vittime umane, giacchè la voluttuosa Tahiti sacrificava uomini all' ira de' suoi Dei.

Le immagini o toa, grossolane e ridicole, si riducevano, per lo più, ad un'allegoria della divinità; tale era il maro ourou, cintura sacra, formata di penne rosse, che solo i grandi maestri, ed in alcune circostanze i Capi, potevano cingere. Essa simboleggiava il Dio del fuoco, rendendo sacro ed inviolabile chi la portava. Strano é, che per procurarsi quelle penne, si doveva poter cogliere l'uccello vivo, strappargliele e lasciarlo libero.

Nelle funzioni religiose i preti recitavano il Rohu Atua, accompagnato da canti, e dovevano essere ben precisi in ogni parola, chè il menomo errore, triste presagio, li obbligava a ricominciare.

Il sacerdozio implicava privilegi estesi, fra cui quello della poligamia, mentre al comune dei mortali non era lecita che la bigamia. Il prete, interprete della volontà divina, era altresì un valido appoggio per i Capi, con i quali generalmente aveva legami di parentela. Ma non era egli solo ad usufruttare la buona fede delle turbe; formidabili concorrenti aveva negli ispirati o profeti, e negli incantatori - assai pericolosi - per gabbare i credenzoni.

Questo popolo, che non aveva scrittura, possedeva, oltre il Rohu Atua, l'Aufau fetii, cioè la genealogia della famiglia.

I Capi ed i proprietari curavano gelosamente cosiffatta tradizione, testimone parlante dei diritti alla sovranità e dei possessi patrimoniali, e la insegnavano di buon' ora a' loro figli, i quali con essa potevano rintracciare i loro antenati più lontani.

Per rammentare fatti storici salienti si adoperavano frasi allegoriche, e per comunicare circostanze individuali od anche eventi 
pubblici, si davano nuovi nomi. A quest'ultimo metodo è dovuto il nome di Pomaré, che vuol dire tosse notturna, perchè il sovrano per alcune notti aveva sofferto la tosse. I Pehe, canzoni di carattere nazionale, domestico o individuale, completavano la storia orale del paese.

La cinica massima dei despoti: pane, armi, feste, non trovó forse in nessun luogo più larga applicazione che nella Polinesia.

Al pane provvedeva lautamente la ricchezza esuberante del suolo. Quanto alle feste, se la ragion di Stato le promuoveva, l'amore per i piaceri del polinesiano, le faceva fiorire; frequenti e sontuose, la loro importanza era tale, che financo le guerre si sospendevano, per celebrarle degnamente.

La piroga sacra, compagna indivisibile di ogni festa, era il punto di raccolta, l' insegna delle gioie pubbliche, ed anche l'emblema tutelare delle spedizioni armate. Portava con essa le vittime e le offerte trovando poi collocamento, nel marae, vicino alle bandiere rosse, segnacoli di pace.

Il Governo aveva forma quasi feudale non priva del tutto di costituzioni semi-democratiche; i Grandi Capi Arii, Rahi erano i signori della Tribí, i quali ripetevano l'autorità suprema dall'elezione popolare, che doveva cadere sempre sopra uno dei membri della loro famiglia. La loro persona come ogni cosa loro propria era sacra, mo'a; seguivano: i Capi inferiori, Tadona; i proprietari, Raatua; ed infine il popolo, istrumento cieco dei suoi distinti padroni.

In mezzo a queste varie caste appariva altera e terribile la famosa setta degli Airoi, i nihilisti polinesiani, la cui origine si perde nell'oscurità della loro cronologia.

Oro, primogenito di Taaroa, scende sulla terra, a Borobora ${ }^{1}$ per cercarvi moglie tra le figlie degli uomini; e, scelta Vairau Mati, la fa madre. Intanto i fratelli di Oro lo raggiungono ed 
affascinati dalla bellezza di Vairau Mati, offrono a lei penne rosse, parte di loro stessi, ed una troia, che nella notte partorisce sette maiali, il secondo dei quali con la cinta rossa degli Airoi. Allora Oro va al gran tempio di Raiatea, e vi depone il maiale della cinta rossa, dicendo: Prendete il maiale degli Airoi; e siate tutti Airoi; io non posso piu esserlo perchè sono padre.

La setta degli Airoi aveva in certo qual modo sancito il principio dell'uguaglianza, chiunque potendo ascrivervisi, purchè facesse il voto di non avere mai prole, o di ucciderla avendola. Erano guerrieri, a cui bastava il solo merito per primeggiare nel sodalizio, che, nella sua gerarchia, contava sette gradi o categorie diverse.

Codesta associazione impressionò tanto i primi navigatori, che diedero il nome di isole della Società a tutto l'arcipelago in cui base del giure pubblico era il diritto del taglione conferito a tutti; però la grande legge della Polinesia, il mezzo, cioè, più acconcio per l'esercizio del potere riscontravasi nel tapiu. Fare una cosa tapi, animata oppur no, significava renderla sacra e nel contempo condannarla all'inerzia. Per le donne, povere paria della societá polinesiana, tutto era tapiu, e dappertutto, eccetto a Tahiti, ove la mitezza del clima e la generosità della terra avendo ingentiliti i costumi, gli uomini non erano così crudeli verso il sesso che tanto contribuiva a fare lieta la loro esistenza.

Le rivalità fra la gente dei monti e quella delle rive del mare, i dissensi di ogni genere fra i piccoli ed i grandi Capi, le guerre, coronamento della cupidigia e malvagità comune, assorbivano in grandissima parte la vita del popolo polinesiano.

Le battaglie erano feroci, specialmente le battaglie navali, in cui le piroghe nemiche si accostavano vicendevolmente e si legavano insieme, perchè la gente combattesse corpo a corpo, senza dare alcun quartiere. Le armate si componevano di piroghe gemelle, che i Capi ordinavano sopra una doppia fila di fronte; la prima linea impegnava il combattimento e lo sosteneva acca- 
nitamente, la seconda fuggiva, quasi sempre, al primo indizio di sconfitta, e prendeva velocemente terra, per assumere la difensiva sulle alture.

Non di rado un banchetto nefando consacrava le orribili carneficine.

I figli di Tahiti, nelle loro lotte intestine, spiegavano minore ferocia; turbolenti anch'essi, tenevano peró in maggior pregio la propria esistenza, e, per non arrischiarla soverchiamente, erano meno solleciti ad offendere quella degli avversari.

La famiglia, quale da noi è intesa, non esisteva nella Polinesia, dacchè il matrimonio, facile a contrarsi ed altrettanto a sciogliersi, non aveva valore morale per alcuno, mentre era solo utile alle caste privilegiate, le quali ravvisavano in tale istituzione l'espediente migliore per avere eredi, e poter salvare i privilegi dei parenti.

Ma la nascita di un bambino era in ogni caso un avvenimento di gran rilievo: la madre doveva ritirarsi con il neonato in una piccola capanna, lontana dal tetto coniugale, ove entrambi diventavano tapir. Alcune settimane dopo, conducevasi il bambino al marae, per circonciderlo ed imporgli il nome del padre o della madre, circostanza questa essenziale, imperocchè nel caso di divorzio dei genitori, egli doveva essere raccolto dai parenti di cui portava il nome.

I Capi, per assicurare l'eredità ai figliuoli, usavano farli riconoscere in loro vece dalla tribir fin da bambini, e da quel momento governavano in nome del giovane signore.

La guerra, per buona sorte, non era opera di ogni giorno nè le feste potevano eternamente durare, sicchè esisteva una tregua alle grandi agitazioni delle masse in cui si chiarivano le abitudini normali del popolo. Quali fossero, lo diremo brevemente rispetto a Tahiti.

Tutti: uomini, donne, vecchi e giovani, si alzavano di buon mattino per recarsi alla riviera, prendere il bagno, e poco dopo ritornare a casa, ove li attendeva la colazione. Il lavoro succedeva 
a questo primo pasto e durava fino al tramonto del sole, ora del pranzo. Piu tardi i tahitiani si abbandonavano ad uno dei loro passatempi prediletti, il cicaleccio che, animato sempre, cessava soltanto quando, coricati tutti da un pezzo, il capo di casa dava il segnale della preghiera e del sonno.

I cibi si componevano di legumi e tuberi - 20 e piu specie, di pesce e di maiale, e le donne erano esclusivamente destinate ad apparecchiarli.

Il pesce si mangiava crudo, non già la carne di maiale, che, con alcuni legumi, era sempre cotta nell'umu, il forno canaco.

Il lavoro consisteva, per gli uomini, nella confezione delle proprie armi, utensili e case. Queste ultime, provvedute di un'ossatura di legno forte, intrecciata con bambi, e di un tetto ricoperto di foglie di pandano, erano solidissime e ben difese dalle intemperie. Esse avevano per mobili: guanciali di legno; sedie di un solo pezzo, tanto più massiccie per quanto il proprietario era di maggior distinzione; gusci di cocco lavorato; un mortaio di legno; un gran piatto di legno; un baule formato da un tronco scavato. Le pareti erano adorne con fionde, giavellotti e mazze, uniche armi del tahitiano; ed il suolo era ricoperto di foglie e stuoie.

Nella ripartizione generale del lavoro spettava alle donne, oltre il preparare i cibi ed assettare la casa, tessere stuoie con il pandano ed il purau, lavorare stoffe con la corteccia di talune piante tessili, e tingerle mercè colori vegetali.

Gl'indumenti indigeni erano il parau, pezzo di stoffa, che avvolgeva il corpo dai fianchi alle ginocchia, ed il tiponta, specie di pianeta, che, sestuplicata, serviva ai guerrieri di corazza.

Il tahitiano, meno dei suoi fratelli polinesiani, usava il tatuaggio, per abbellirsi o incutere timore; l'uomo non si tatuava il viso, e la donna si limitava a tatuare i piedi, le mani ed altre parti del corpo piú raramente scoperte.

E da osservare che nell'estetica indigena la pinguedine delle donne era elemento di bellezza, come lo è oggi ancora in Oriente, e come lo fu, in passato, fra gli israeliti. 


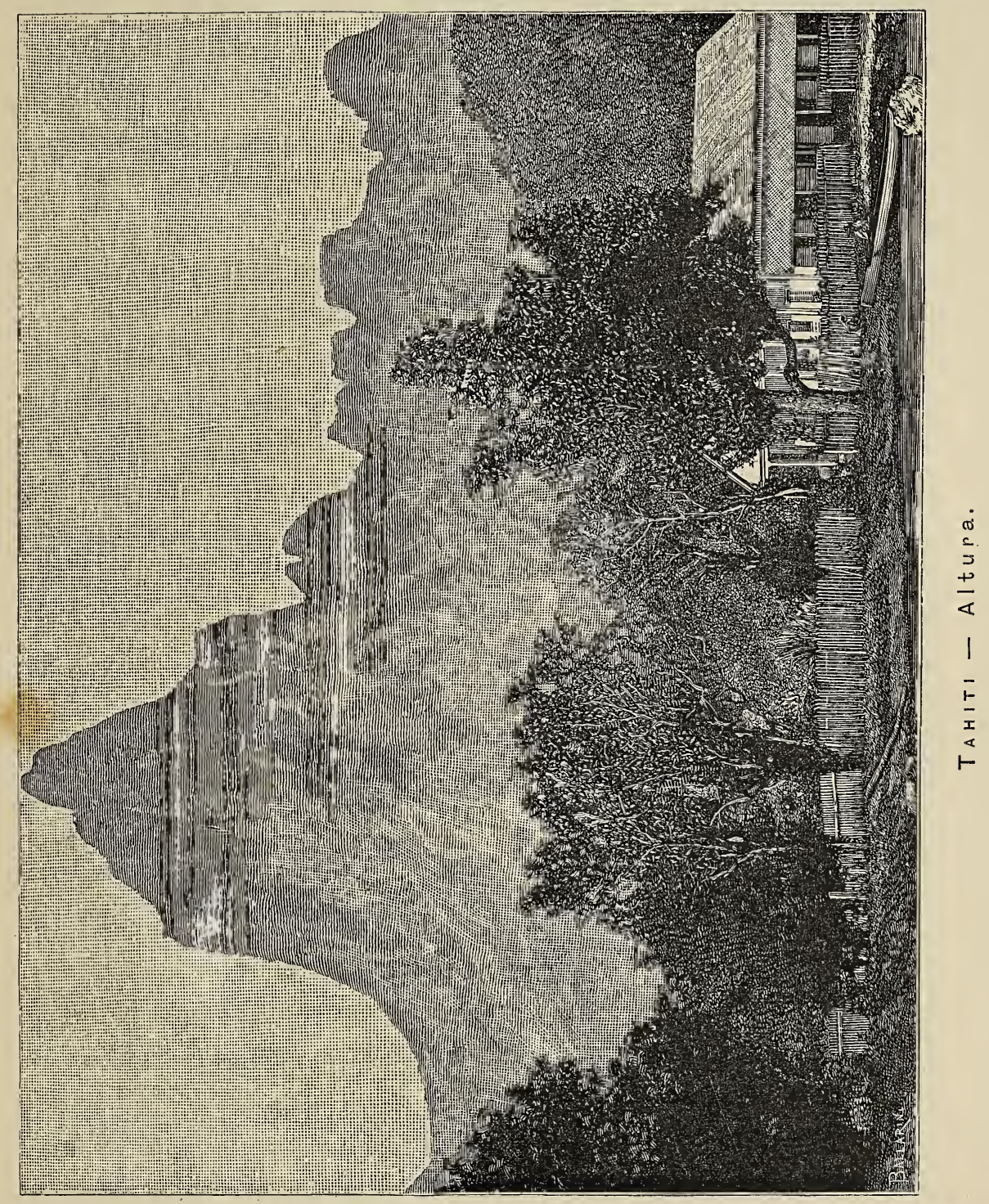



I tahitiani non ebbero mai nè un Esculapio, nè un Ippocrate, dacchè per essi le malattie non potevano avere altra causa se non la vendetta degli Dei, de' morti, o le arti malefiche degli incantatori. Si curavano quindi a norma dei responsi del tihoua toutera, l'ispirato. Accorrevano i parenti presso il malato, per piangere ed offerire doni, e s'egli era un Capo, il popolo tutto recavasi da lui con lo stesso intento. Gemiti, grida di desolazione segnalavano la morte. Il defunto era portato, sopra un altarino, al tempio e, fatto colá disseccare, dopo estratti gl' intestini, lo si seppelliva nella tomba della famiglia, grotta nascosta nelle montagne.

La monotonia dei giorni placidi, gli incidenti disgustosi inerenti alla vita umana trovavano, fra codesta gente dall'insaziabile allegria, compenso, in difetto di feste pubbliche, nei divertimenti privati, ritagliati sul tempo dedicato al riposo: danze, rappresentazioni teatrali, canti.

II.

\section{Scoperta della Polinesia - Conseguenze.}

Era sentenza dei tempi, che una razza ardita, amante di novità, intelligente, forte, sarebbe apparsa in codeste terre della Polinesia, per abbatterne gli Dei, mutare i costumi dei suoi figli e, chi sa? lasciare vuoti, se non confusi, con il mutamento, la loro mente, il loro animo. L'apparizione ebbe luogo nel 1767.

Wallis, al comando del Dolphin di S. M. Britannica, per il primo visitó l'isola di Tahiti, ed un anno piú tardi la riconobbe Bougainville, al comando della Boudeuse, francese, chiamandola, straordinariamente colpito da' costumi della sua popolazione, la Nouvelle Cythère, oic l'amour sans pudeur, n'est pas sans innocence. 
Cook vi approdava, nel 1769, con gli scienziati che secolui dovevano a Tahiti stesso osservare il passaggio di Venere, sbarcando a Matavai ${ }^{1}$ e stabilendo il suo osservatorio sulla punta di terra, che prese e conserva il nome dell'astro citato.

Taluno crede che il navigatore spagnuolo Quiros, partito dal Callao sullo scorcio del 1605, avesse avvistato l'isola di Tahiti il 9 febbraio 1606 e le avesse imposto il nome di Sagittaria; ma altri invece sostiene che la descrizione fattane da Quiros non corrisponde all'isola di Tahiti. Peraltro, ove la cosa abbia pure qualche importanza in ordine alla cronologia, non ne ha alcuna per la storia della Polinesia, e solo merita qui menzione per debito d'imparzialità.

Ai tempi di Wallis regnava in tutto lo splendore della potenza, sulle isole Tahiti e Moorea, come Grande Arii, Amó, la cui nobile famiglia aveva, supponesi, esercitato in quelle terre per 150 anni almeno, un potere incontestato. Moglie di Amò era Bena, la Regina, che ispirava, per le sue doti eminenti, sommo rispetto e venerazione.

Lontano dal litorale, Amò vedeva raramente i bianchi, e quando volle far riconoscere Grande Arii suo figlio, si trovò di fronte, pretendente al trono, Vaiatua, il futuro Pomarè, che Toutaha, Capo arricchito da Bougainville, aveva istigato alla ribellione. Amò fu sconfitto, ed il dominio delle Isole passò nelle mani di Vaiatua.

Giunsero allora a Tahiti missionari wesleyani, a cui fecero buon viso perfino i sacerdoti indigeni.

Nel 1803 Pomarè I, volendo appropriarsi il dio Oro del marae di Ato hou rou, deve sostenere una guerra micidialissima, dalla quale esce vittorioso soltanto in grazia déll' intervento dei bianchi.

Muore in quell'anno, ed alla sua morte gli succede al trono, senza alcun contrasto, il figlio Otou (Pomarè II), che avrebbe abbracciata la religione cristiana, se due guerre, a breve intervallo l'una dall'altra, non avessero assorto tutti i suoi pensieri.

${ }^{1}$ È un ancoraggio al N. E. di Papeete. 
Fatto nuovo negli annali tahitiani, la seconda di queste guerre è provocata dal rapimento di una donna, della bella moglie del valoroso Capo Metonavi, per parte di un ufficiale di Pomarè. Ma l'Elena tahitiana non porta fortuna alle armi di Pomarè, che, battuto due volte, e due volte costretto a ritirarsi, si risolve a chiedere al cristianesimo i conforti e l'aiuto negatigli da' suoi Dei.

Torna alla riscossa Pomarè, nel 1815, e sconfigge il nemico, proibendo la carneficina dei vinti, che era in passato il coronamento di ogni vittoria. Quest'atto di clemenza del Principe gli cattiva la simpatia generale, e diventa il segnale della conversione religiosa di molti isolani.

Manca ai vivi Pomarè II, nel 1821, lasciando erede un figliuolo di 3 a 4 anni, che muore sei anni dopo; e la sovranità viene assunta dalla sua sorella Aimata, con il nome di Pomarè IV.

L' Isola da quel giorno entró in una fase di scandalosi intrighi politici e religiosi, che dovevano togliere, per sempre, ai bravi isolani l'antica serenità sì facile e naturale, ma del resto già scossa dagli esempi di dissolutezza offerti dai disertori della Bounty. ${ }^{1}$ E nacquero ribellioni contro il potere supremo, susseguite da violente repressioni, e prese consistenza la setta Mamaia, tendente a ripristinare le vecchie usanze, le vecchie credenze.

In mezzo a codesto caos d'idee, di costumi, di governo, l'affluenza maggiore di navi mercantili europee, nei mari oceanici, preparava intanto alla Corona tahitiana difficoltà di un nuovo genere e gravi: difficoltà internazionali, che si tradussero, nel 1835, nella richiesta del pagamento di 2850 piastre fatta dal Comandante. inglese Fitz Roy, a titolo d'indennità, per la cattura ed il saccheggio di una nave di quella nazione, atti ambidue imputabili agl' indigeni di Tuamotu.

${ }^{1}$ L'equipaggio della Bounty di S. M. britannica si ammutinò nelle acque di Tahiti; affondò la propria nave dopo avere imbarcato il Comandante in una lancia, abbandonandolo alla ventura. Esso si sparse in varie isole dell'arcipelago di Tuamotù e nell'isola stessa di Tahiti. 
A turbare maggiormente i sonni della arrendevole Regina, si unirono tosto agl'interessi commerciali stranieri il dualismo delle due Chiese importate a Tahiti, protestante e cattolica. La Corte, parteggiando per la prima di esse, venne in suo aiuto col far decretare dalla Sovrana l'espulsione dai suoi dominî dei missionari cattolici. Ma gli espulsi erano francesi, e l'ammiraglio Dupetit Thouars, che comandava le forze navali di Re Filippo, in Oceania, informato del fatto, accorse a Tahiti, per esigere, a sua volta, indennità e riparazioni d'onore.

Il Governo tahitiano non comprendeva certo, come cotali gare religiose potessero ripercuotersi, per aizzarle, sulle naturali gare politiche de' Paesi, a cui appartenevano gli autori dei dissensi teologici, e non immaginava quindi che nei vortici di queste ultime potevano naufragare e la libertà di Tahiti e la sua autonomia. Ignorante, era spensierato e, con la spensieratezza, fedifrago, titubante; e quando, a furia di errori, cadde avvinto dal piu forte, dovè irremissibilmente subirne le strette.

Dupetit Thouars aveva lo sguardo penetrante, profondo il sentimento della dignità nazionale, ed era ardito; onde non esitó, tuttochè sapesse d'imbattersi nei dubbi del timido Governo di Guizot, ad affermare la supremazia della Francia a Tahiti, perno dell'Oceania, ove si era tentato ledere i diritti dei suoi concittadini, e colse l'occasione di violati patti per costringere la Sovrana dell' Isola a desiderare e volere il protettorato del di lui Paese.

Tahiti da mezzo secolo non era più la terra degli incanti giovanili; la corruttela aveva invecchiato il suo popolo, che, con i recenti vincoli di schiavitu straniera, cadeva nella decrepitezza, per dare così ragione alla profezia di Oro: $E$ tu pu te fano, $e$ toro te fare ro, e moa te taata. (L'ibisco nascerà, la madrepora si diramerà, ma l'uomo cesserà).

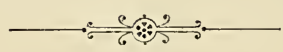




\section{CAPITOLO III.}

LINGUA DFLIA POLINHSIA 1

La lingua polinesiana, sia che la si consideri come madrelingua o come avente una comune origine con la lingua malese, è certamente di una grande antichità, poichè il popolo che la parla, e che ha creduto fino a quest'ultimi tempi essere il solo popolo esistente, dovè rimanere per lunghe età separato dal resto del mondo.

$\mathrm{E}$ mentre in molte parti essa è deficiente, com'è da aspettarsi trattandosi del linguaggio di gente rozza e barbara, non manca, per certi rispetti, di possedere una forza, una semplicità ed una precisione, come ad esempio ne' pronomi personali, che la colloca ad un alto livello.

Le sue rassomiglianze con l'ebraico nelle coniugazioni dei verbi ed in molte delle sue parole primitive, possono essere dimostrate; molte parole sembrano avere veramente radici ebraiche, tali come: mate, morte; mara o maramara, amaro; rapaau, sanare; pae, lato, ecc.

Siccome il polinesiano prevale sopra una vastissima distesa dell'Oceano Pacifico, ed è parlato da popolazioni per la maggior

${ }^{1}$ Consultare per maggiori particolari il Tahitian and English dictionary, 1851, e le Transactions of the Philological Society, 1877-79. 
parte abitanti piccole e staccate isole, con poca o nessuna relazione fra di loro, così presenta una grande varietà di dialetti, che non sono così diversi da non essere riconosciuti come appartenenti ad un solo comune linguaggio.

Fra questi dialetti i principali sono: il samoano, l'havaiano o delle isole Sandwich, il marchesiano, quello della Nuova Zelanda, il tongatabuano, o delle isole degli Amici, ed il tahitiano. Gli altri, sebbene poco conosciuti, mostrano tutti maggiore o minore affinità coll'uno o coll'altro dei citati dialetti.

$\mathrm{Vi}$ è nella lingua polinesiana un gran numero di radicali o parole primitive, che sembrano primeggiare in tutti i dialetti, per avere la stessa pronunzia e significare la medesima idea: mate, morte; vai, acqua; ua, pioggia; feana, paese; tai, mare; uta, costa; metua, parente; atua, Dio, ecc.

Altri vocaboli come i pronomi personali, numerali e possessivi sono quasi simili in tutti i gruppi e, probabilmente, lo stesso può dirsi dell'uso degli aggettivi e della coniugazione dei verbi. Con tutto ciò, molte parole differiscono assai piu in apparenza che in realtá, perchè in alcuni dialetti la prima sillaba di una parola è soppressa o cambiata: $t$ è preso per $k, h$ per $f, n$ per $n g, l$ per $r$ o il contrario; così la parola uomo (persona umana) nell'havaiano è kanaka, parata nelle Marchesi, tongata nel tongatabuano, tamata nei dialetti della Nuova Zelanda, di Samoa e delle Figi, mentre nel tahitiano è taata.

Gita è il vocabolo che nei diversi dialetti esprime pesce, ma a Tahiti si dice $i a$; anche buoka, che significa maiale, è detto puaa in tahitiano. $R a$ è il nome generico dato al Sole, ma alle Marchesi si dice $a$, e nelle isole degli Amici $l a$.

Ariki o Aiki dinota un Re o un Capo di tribù, dappertutto, mentre i tahitiani dicono Arii.

I dialetti accennati, che conservano maggiore rassomiglianza fra di loro sono l' havaiano, il marchesiano e quello della Nuova Zelanda; il tahitiano viene dopo, e differisce principalmente da questi per l'abbreviazione delle parole, la soppressione di un 
gran numero di consonanti, e per l'assoluta mancanza del nasale $u g$, del $g$ e del $k$.

Il dialetto tongatabuano è diverso dagli altri, sotto vari rapporti; esso sostituisce la $l$ alla $r$, ed usa l' $f$ come consonante, ciò che non fanno mai gli altri dialetti; ha pure forti aspirazioni, ed ha un gran numero di parole sconosciute altrove, ma che si possono facilmente rintracciare, nei dialetti usati alle Figi, Nuova Caledonia ed alle Marianne o isole dei Ladroni. Nulla nel dialetto tongatabuano autorizzerebbe a sostenere la congettura che i neozelandesi siano loro discendenti.

È da aggiungere che alcune tribú polinesiane mutarono recentemente la pronunzia di una o due consonanti. I nativi di Minè (isola dei Selvaggi) cambiarono il $t$ in $t$.

Vent'anni fa una parte soltanto della popolazione più giovane sostitui il $t s$ al $t$ davanti alla vocale $i$, come lautsi per lauti, ma piu tardi l'esempio fu imitato da tutti, ed oggi molti adoperano il $t s$ davanti a tutte le vocali.

I dialetti polinesiani hanno una grande similitudine, nel suono e nelle forme grammaticali, con i dialetti malesi, dell'arcipelago indiano, e di Madagascar. Perciò, in complesso, sembrerebbero derivare da uno stipite comune, quantunque riesca difficile, se non impossibile, scorgerne oggi la menoma traccia.

Il malese poi è forse la lingua, che per le successive trasformazioni subite, si discosta maggiormente dal primitivo tronco; e, fuori dubbio, è quella che gl'influssi stranieri, scesi dalle Indie, ed il contatto con gli arabi, travolsero di piu.

Egli è certo che i malesi-polinesiani furono separati dai loro congeneri dell'arcipelago Indiano assai prima che i citati influssi intaccassero la lingua malese. Quindi, tenuto conto della similitudine esistente tra i vari dialetti malesi-polinesiani, e del loro isolamento e relativa semplicità, si verrebbe a conchiudere, che uno studio comparativo di essi permetterebbe forse di ricostituire la lingua madre, da cui tutti si allontanarono, prendendo vie divergenti. 



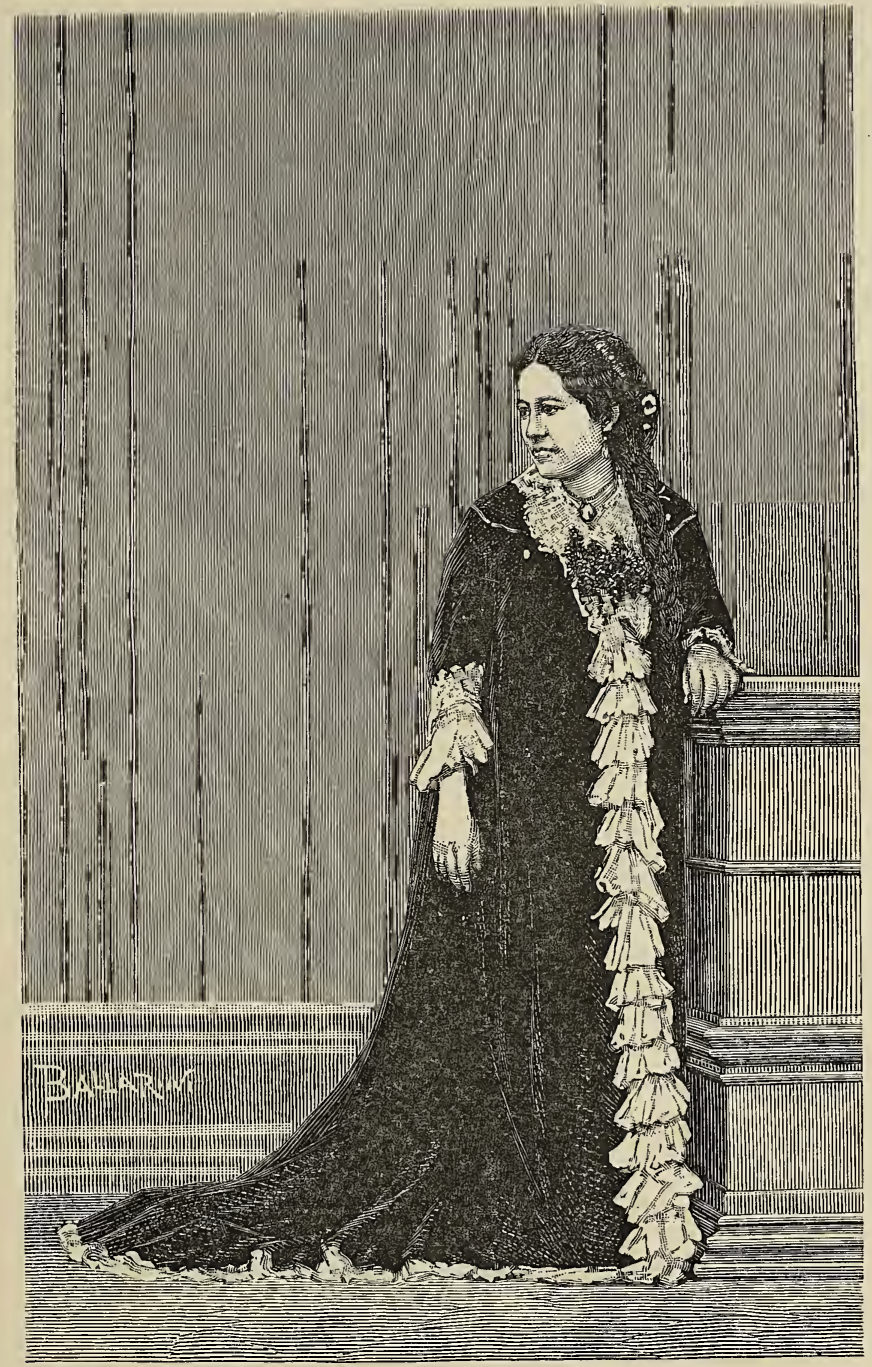

HaLF $C_{A S T}$, figlia di padre inglese e di madre indigena (costume dell'aristocrazia). 



\section{CAPITOLO IV.}

ORDINAMENTO POLITICO

I.

Divisione politica.

La Francia ha dato il nome di Stabilimenti francesi nell'Oceania agli arcipelaghi ed isole dell' Oceano. Pacifico Australe, che seguono :

Arcipelago delle isole della Società.

Arcipelago delle isole basse o Tuamoti.

Arcipelago delle Marchesi.

Isole Gambier.

Isole Tubuai o Raivavae.

Isole Rapa.

L'arcipelago delle isole della Società è diviso in due gruppi: di S. E., detto anche delle Isole al vento, e di N. O., detto delle Isole sottovento.

Il primo gruppo, formato dalle isole Tahiti, Moorea e dagli isolotti Tetiaroa e Meetia, è annesso alla Francia.

Il secondo gruppo, formato dalle isole Huahine, Raiatea, Borabora, ed altre piccole, in forza di una dichiarazione reciproca, fatta 
nel 1847, dalla Francia e dall' Inghilterra, conserva la sua indipendenza. ${ }^{1}$

L'arcipelago di Tuamotú, che comprende 78 isole, è annesso alla Francia.

L'arcipelago delle Marchesi, di 11 isole, è dipendenza francese. Le isole Gambier, cioè cinque isolotti racchiusi in una medesima cinta di coralli, sono annesse alla Francia.

Le sole Tubuai e Raivavae, dello stesso arcipelago, e l'isola Rapa, sono dipendenze francesi. ${ }^{2}$

II.

Protettorati - Annessioni - Prese di possesso.

Fu nel settembre dell'anno 1842 che la regina Pomarè IV, di Tahiti, propose al Contr'ammiraglio Dupetit Thouars, comandante di una divisione navale francese nelle acque di Tahiti, di sollecitare il Re dei francesi a voler prendere sotto la sua pro tezione il di lei regno, per non essere esposta - diceva la Regina -

1 Raiatea e dipendenze (l'isola Tahaa) chiesero nel 1880 il protettorato francese, e ne adottarono la bandiera. Se la Francia abbia o no accettata la domanda egli è ciò che non abbiamo potuto sapere in modo preciso; l'annuario ufficiale di Tahiti del 1883, che fa accurata menzione di tutti gli stabilimenti soggetti, in una guisa o nell'altra, alla Francia, tace del protettorato in parola. Ma abbiamo veduto la bandiera del protettorato francese alzata all'albero di maestra da una nave da guerra francese, che aveva a bordo la Regina di Raiatea.

${ }^{2}$ Non risulta da alcun documento pubblico come e quando queste isole divennero parte integrante de' possedimenti francesi. 
a perderlo assieme con la libertà e l'autorità, non potendo oltre governare da sè, nel presente stato di cose, in modo da conservare la buona armonia con i Governi stranieri.

L'Ammiraglio francese annui, in nome del Re, alla proposta fattagli, salvo ratificazione, che ebbe luogo il 25 marzo dell'anno 1843.

Nel marzo del 1880 il Re Pomarè V, figlio della Regina Pomarè IV, notificando pubblicamente a' membri della famiglia reale, a' Capi ed al popolo degli Stati del Protettorato la sua intenzione di visitare, prossimamente, le dipendenze di Tahiti, dichiarava di rimettere, durante la sua assenza dalla capitale, il governo e l'amministrazione dei suoi Stati, ed in generale tutti i suoi diritti sovrani, nelle mani del Commissario della Repubblica.

Questo avvenimento ch'ebbe tutte le apparenze di un esperimento di annessione, era seguito tre mesi dopo dall'unione di Tahiti e sue dipendenze alla Francia, con l'immediata adesione del Rappresentante del Governo francese.

Il protettorato nelle isole della Società e di Tuamotù aveva così durato 38 anni.

I Sovrani di Tahiti, in ogni tempo, fecero prevalere la loro influenza dominatrice nell'arcipelago di Tuamotú; e Pomarè I, sullo scorcio del secolo passato, per accrescerla con la leva delle credenze religiose, mandó ad Anaa, isola fra tutte la più importante, missionari protestanti, partigiani delle sue vedute politiche.

Gli abitanti dell'Arcipelago, legati alla dinastia tahitiana, per i benefizi ottenuti dalla presenza di quei missionari, vollero associare le loro sorti a quelle di Tahiti, quando la regina Pomarè IV implorò il protettorato francese, per salvare il trono, ma pretesero, ed a buon diritto, che la denominazione di Isole di Pramoti, che significa Isole sottomesse, imposta in altri tempi da'tahitiani vincitori o conquistatori, fosse cambiata in quella di Isole di Tuamotu, che vuol dire Isole basse.

La presa di possesso dell'arcipelago delle Marchesi, in nome della Francia, si effettuò dal Contr'ammiraglio Dupetit Thouars il $1^{\circ}$ maggio 1842, riguardo all'isola Tavata del gruppo di S.E., 
ed il 2 giugno dello stesso anno, riguardo all' isola Nuka-Iva del gruppo di N. O. Una legge dell' 8 giugno 1855 designava l'Arcipelago a luogo di deportazione, ma eccettone tre persone, che furono poi graziate, essa non venne mai applicata.

Le isole Gambier invocarono il protettorato della Francia nel febbraio 1844, facendo il Re e i Grandi Capi professione di fede cattolica, e chiedendo di voler formare uno Stato libero e indipendente, sotto la protezione di S. M. Filippo, Re dei francesi. Toccò ancora all'Ammiraglio Dupetit Thouars ${ }^{1}$ di accogliere favorevolmente la domanda degli isolani di Gambier, con riserva di ratificazione sovrana, che venne in seguito accordata. Nel febbraio 1881, per volontà dei detti isolani, era fatta rimessione completa alla Francia di tutti i poteri devoluti al Re ed ai Consigli del Paese.

III.

Ordinamenti indigeni sotto il Protettorato.

Sotto il protettorato un Commissario del Governo francese aveva incarico di trattare ogni questione di carattere internazionale del Governo tahitiano, e gli affari concernenti i francesi e gli stranieri residenti negli Stati del Protettorato. Le sue relazioni con le autorità indigene erano regolate da decreti e leggi tahitiane.

Un piccolo drappello di lancieri indigeni, reclutati tra' figli dei Capi, formava una specie di milizia d'onore del Governo tahitiano. Questi cavalieri, comunemente adibiti alla trasmissione degli

${ }^{1}$ La domanda del protettorato fu fatta direttamente al Capitano di vascello cav. C. Pénaud, comandante la fregata Charte della divisione dell'Ammiraglio Dupetit Thouars. 
ordini del Governo, accompagnavano il Commissario francese nelle sue gite ufficiali; non portavano altra arme che la lancia.

Vi era un'assemblea legislativa, che il Sovrano ed il Commissario francese convocavano annualmente con decreto, e che si componeva: di deputati eletti per tre anni, con suffragio universale e diretto; dei Capi di distretto eletti a vita; dei proprietari nominati dalla Corona; e dei Toohitous, ossia grandi giudici, di nomina sovrana.

Il territorio degli Stati del Protettorato dividevasi in distretti, e la popolazione era raggruppata per villaggi. Ogni abitante doveva possedere una casa tenuta in buone condizioni di pulizia e salubrità. Le popolazioni disseminate e poco numerose riunivansi agli abitanti del distretto piu vicino per formare il villaggio o comune.

Il villaggio era amministrato da un Consiglio, con attribuzioni molto late, e formato: dal Capo, presidente, dal deputato, e da tre consiglieri titolari (eletti dagli abitanti), dei quali uno segretario.

Ogni villaggio doveva avere una scuola, ed ogni distretto una casa municipale (Chefferie), una prigione, una Faré hau (casa delle riunioni pubbliche) ed un tempio.

Nelle isole del Protettorato il Governo era molto tollerante circa l'applicazione di queste prescrizioni, ed i Consigli si formavano mano mano che gl' indigeni si mostravano atti a far parte della generale organizzazione.

In ogni distretto vi era un giudice, eletto da' proprietari e scelto dal Sovrano, che rendeva sommariamente giustizia sopra taluni delitti previsti dalla legge.

Il Consiglio del distretto interveniva per autorizzare o rifiutare dinanzi al giudice l'azione penale, per delitti o contestazioni. In caso di opposizione del giudice, il Consiglio ne riferiva alla Corona.

V'era appello contro i giudizi del giudice del distretto secondo i casi contemplati dalla legge, ad un Tribunale di appello sedente a Papeete. Questo Tribunale, che era permanente, si componeva di tre giudici e di un segretario. Il Governo si faceva rappresen- 
tare da un delegato, che areva soltanto voce consultiva. I giudizi di questo Tribunale non erano definitivi che nel solo caso, in cui la parte condannata non avesse ricorso all'alta Corte (Corte dei Toohitous), nello spazio di tempo fissato dalla legge.

L'alta Corte indigena aveva la sua sede a Papeete e teneva tre sessioni per anno: in gennaio, maggio e settembre.

L'alta Corte giudicava in ultimo appello tutte le cause, le quali essendo state giudicate dal Tribunale di appello, erano portate dinanzi ad essa, a termini di legge.

Le cause relative alle terre ovvero ai titoli provenienti dagli antenati, erano dapprima portate davanti al giudice, assistito da un certo numero di houiraatiras (proprietari). Se una delle parti non accettava il giudizio, essa poteva appellarsi al Tribunale sedente a Papeete ed, in ultimo, all'alta Corte.

Un ordine del Sovrano e del Commissario del Governo francese denunziava alla Corte dei Toohitous, tutti i delitti passibili della pena di morte.

I giudizi dell'alta Corte potevano, per decisione della Corona o del Commissario francese, essere cassati per violazione delle forme sancite dalla legge. La stessa decisione ordinava, che la causa sarebbe stata giudicata nuovamente.

Nell'alta Corte il Governo si faceva rappresentare da un delegato avente voce consultiva solamente.

Il servizio di pubblica sicurezza era affidato in ogni villaggio ad un mutoi, ed in ogni distretto ad un mutoi imiroas, entrambi scelti dal Consiglio del distretto.

Nel capoluogo, a Papeete, la polizia generale apparteneva alla gendarmeria francese coadiuvata da un capo mutoi, il quale disponeva di alcuni muto $i$, incaricati di sorvegliare specialmente gli indigeni e gli stranieri di origine oceanica. 
IV.

\section{Ordinamenti coloniali e indigeni dopo l'annessione.}

Dopo la rinunzia al trono fatta da Re Pomarè V e la conseguente annessione del suo regno alla Francia, l'ordinamento politico, giudiziario ed amministrativo in vigore sotto il Protettorato si è, per così dire, mantenuto intatto nella sostanza, se non perfettamente nella forma; e le innovazioni che successivamente furono introdotte nel governo generale del paese, mirarono a dare alla Colonia francese un assetto vieppiù conforme ai suoi bisogni, senza cambiare l'essenza delle leggi indigene, onde non contrariare usi e costumi ormai profondamente radicati ed insiti in quelle popolazioni.

Nel giugno 1880 il Commissario della Repubblica, assunto il titolo di Governatore degli Stabilimenti francesi nell' Oceania, avocò a sè la trattazione di tutti gli affari, esteri ed interni, relativi al funzionamento del nuovo possedimento nazionale, uniformandosi, in massima, allo spirito delle leggi coloniali francesi ed alle esigenze locali.

Fu creata pertanto una Direzione dell' interno, per la polizia generale, per l'amministrazione delle contribuzioni dirette e indirette e per quella delle finanze locali e servizi affini; Direzione che riassume il duplice compito amministrativo e di sicurezza pubblica, appartenente, anteriormente all'annessione, al Governo tahitiano.

Contemporaneamente furono costituiti i seguenti uffici:

Consiglio d'amministrazione della Colonia, il quale con l'aggiunta di alcuni magistrati si trasforma in Tribunale del contenzioso amministrativo; Consiglio coloniale, per suggerire le misure più opportune allo sviluppo della Colonia;

Comitato per le finanze; 
Camera di commercio;

Consiglio superiore d'istruzione pubblica;

Consiglio dei lavori;

Commissione di difesa;

Commissione sanitaria e Consiglio d'igiene e di salubrità pubblica;

Comitato di sorveglianza dei piroscafi.

\section{Esistevano e furono conservati:}

Comitato centrale agricolo ed industriale;

Casa agricola;

Servizio postale fra la metropoli e Tahiti, via S. Francisco di California, a vela; fra Tahiti e gli altri stabilimenti oceanici, con battelli a vela, e nell'isola di Tahiti con vetture pubbliche e corrieri a piedi o a cavallo;

Tesoreria coloniale;

Stamperia del Governo; ${ }^{1}$

Ufficio di curatore dei beni vacanti, di registro, ipoteche e demanio;

Ufficio di ponti e strade;

Servizio del porto e pilotaggio;

Stato Maggiore Generale e della Piazza;

Direzione d'artiglieria;

Ufficio di gendarmeria coloniale;

Consiglio di revisione (giustizia militare);

Due Consigli di guerra permanenti;

Commissariato di marina;

Arsenale di marina;

Stazione navale locale.

Attualmente, il Governo della Colonia provvede all'istruzione primaria con scuole laiche proprie e maestri indigeni ed europei, ma lascia intera libertá alie scuole protestanti e cattoliche, che s'intitolano scuole libere.

L'amministrazione della giustizia, in quanto riguarda i francesi e gli stranieri residenti negli Stabilimenti francesi, nell'Oceania, si svolge per mezzo di un Tribunale superiore, di un Tribunale

1 Dobbiamo alla cortesia squisita del Direttore della stamperia del Governo la comunicazione di molti de' documenti ufficiali, da cui si ricavarono le notizie relative al presente capitolo. 


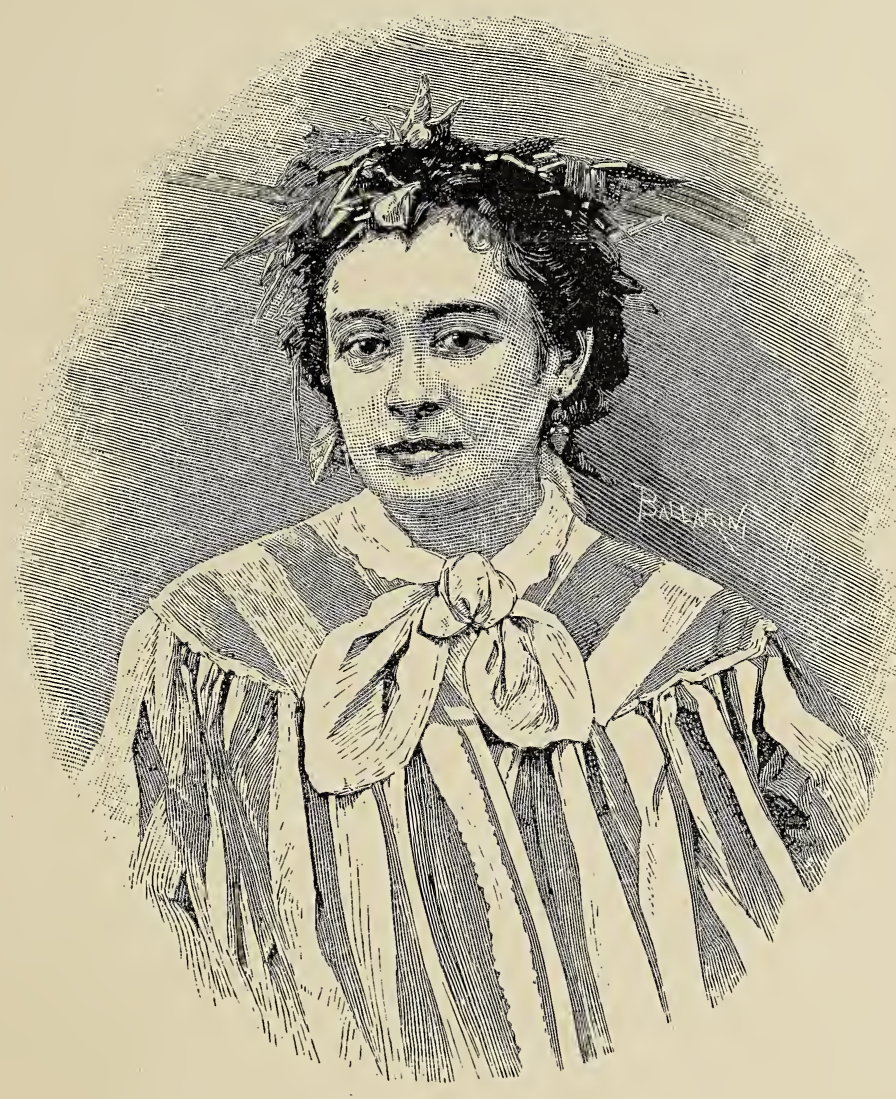

Figlia di una "chefesse" di Moorea (puro sangue tahitiano). 

di prima istanza, di un Ufficio del Procuratore del Governo centrale, di un Tribunale di commercio e di Giudici di pace.

Fuori dell' isola di Tahiti, il Governatore è rappresentato, in ogni distretto principale, da un Residente, che riunisce le attribuzioni di Comandante militare, di Amministratore, di Tesoriere ed in alcuni casi anche di Ufficiale dello stato civile e di Giudice di pace.

Le funzioni di Ufficiale dello stato civile sono comunemente coperte dai rispettivi Capi di distretto.

Sedi di Residenti sono: Papetoai nell'isola Moorea; Rotoara nell'isola Fakarava di Tuamotu; Tubuai nell'isola Tubuai; Taiohae a Nuka-hiva delle Marchesi; Rapa nell' isola Rapa; Mangareva nelle isole Gambier.

Il territorio delle isole Marchesi è ripartito in tribù (Chefferies), i cui Capi, investiti e stipendiati dal Governo degli Stabilimenti, ricorrono alla Residenza in ogni affare importante della propria giurisdizione.

Finora, atteso il depresso livello morale degli indigeni, non fu possibile dare alla famiglia marchesiana un ordinamento un po' elevato, e l'attuale occupazione può considerarsi, più che altro, nominale.

Una Missione cattolica, fondata a Nuka-hiva da parecchi anni, si sforza incessantemente a educarli, ma con insensibile profitto.

Le isole Marchesi, oltre il Residente, hanno un Vice-residente, un Capo del servizio amministrativo, un Capo delle contribuzioni ed un Sorvegliante di strade. Il Governo vi ha due scuole: l'una per maschi, l'altra per femmine, e sussidia alcune scuole libere. Esiste a Nuka-hiva un servizio del porto e del pilotaggio. La forza pubblica e la forza militare sono rappresentate, nell'Arcipelago, da 10 gendarmi e da 46 soldati di fanteria marina, comandati da un tenente.

Le isole Gambier sono divise in quattro distretti, ciascuno dei quali ha un Consiglio, un Maestro di scuola, un Giudice e 
due Assessori, un Capo mutoi ed il numero di mutoi riconosciuto necessario. Il Consiglio si compone di un Gran Capo (Capo del distretto) e di tre Consiglieri.

Le cariche accennate sono il risultato di elezioni fatte, nel rispettivo distretto, da un'assemblea, a cui prendono parte tutti gli uomini di etá maggiore di 21 anno. I titolari però non possono entrare in funzioni senza l'investitura del Residente.

A Rikitea, capoluogo della Residenza, funziona inoltre un Gran Consiglio, o Tribunale di appello, per gli affari esclusivamente indigeni, formato da quattro Grandi Capi di distretto, sotto la presidenza del Residente. I ricorsi in Cassazione degli atti, decisioni o giudizi del Gran Consiglio possono essere presentati al Governatore degli Stabilimenti, in occasione della sua visita annuale alle isole Gambier.

Un Tribunale misto giudica, a norma delle leggi francesi, gli affari fra europei e indigeni.

Gli indigeni hanno facoltà d'invocare l'applicazione delle leggi francesi.

L'Assemblea di distretto, citata sopra, è convocata dal Residente ogniqualvolta egli lo stimi necessario per trattare gli affari che interessano il paese.

L'istruzione primaria è obbligatoria per tutti i fanciulli, e mentre ogni distretto è tenuto ad avere una scuola elementare, il Governo sussidia inoltre, a Rikitea, due scuole superiori professionali per i due sessi.

I lavori di utilità pubblica sono a carico del popolo, e ciascuno ha l'obbligo di contribuirvi, senza retribuzione di sorta, allorchè ne venga richiesto dal Governo.

Il Gran Capo del distretto è l'Ufficiale dello stato civile.

Tutte le disposizioni legislative, concernenti la costituzione politica, amministrativa e giudiziaria del paese, trovansi compendiate in un codice, tradotto dal francese in lingua tahitiana, e promulgato nel febbraio dell'anno 1881.

Completano l'ordinamento generale delle isole Gambier un 
Ufficio di porto, un Comitato di agricoltura, un Ufficio delle contribuzioni ed una Missione cattolica con un Provicario apostolico.

Il Governatore degli Stabilimenti francesi dell'Oceania è comunemente un capitano di vascello od un commissario di grado assimilato, a cui competono gli onori di vice-ammiraglio. Ha sotto la sua dipendenza diretta tutte le forze militari stanziate ne' detti Stabilinienti.

Un avviso, comandato da un tenente di vascello, è, in permanenza, di stazione nel porto della città capoluogo: Papeete. Quattro golette a vela fanno la pulizia degli arcipelaghi e riscuotono le tasse; sono armate di due cannoni da 8 centimetri n. 1 di bronzo, con ventidue persone di equipaggio, al comando di un tenente di vascello.

Ogni anno, in occasione dell'anniversario della Repubblica, il Comandante in capo della divisione o squadra del Pacifico visita, con la propria nave, l' isola di Tahiti, a fine di accrescere lustro alle feste pubbliche, e profitta della circostanza per ispezionare la forza navale dipendente dal Governatore.

Mensilmente, parte dalla Nuova Caledonia, per Tahiti, un trasporto ad elica, armato di quattro cannoni da 14 centimetri, allo scopo di portare viveri, materiale, personale, ecc. all'Amministrazione locale; questo trasporto provvede a' cambiamenti della guarnigione e degli equipaggi delle navi militari. ${ }^{1}$

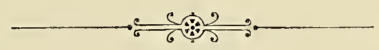

${ }^{1}$ Gl' indigeni colti e indipendenti, che hanno viaggiato - parechi furono educati in Australia - sembrano, a nostro modo di vedere, simpatizzare debolmente con l'Amministrazione francese, a cui addebitano di curare poco gl'interessi tahitiani, e di ritrarre invece per sè esclusivamente ogni vantaggio derivante dal dominio della Francia. Gl'indigeni che rivestono cariche governative sono, com'è naturale, soddisfatti di questo dominio, mentre la massa, il popolino, si mostra indifferente affatto a riguardo della presente forma di giogo straniero, desiderando solo il rispetto della proprietà indigena ed il mantenimento de' tradizionali costumi. 

9

\section{CAPITOLO V.}

\section{PRODUZIONE FCONOMICA}

I.

\section{Popolazione.}

LA popolazione attribuita a Tahiti motivò sapienti controversie da un lato ed attacchi pungenti dall'altro, all'indirizzo dell'illustre navigatore Cook, che l'aveva fatta ascendere, nel 1777, epoca del suo ultimo viaggio, a settanta od ottantamila anime, mentre più che mezzo secolo dopo, quando i francesi presero stanza nell'isola, i suoi abitanti non oltrepassavano i settemila.

Cook era stato accusato d'esagerazione, e di essere caduto in errore, lui così parco ed esatto nei suoi giudizi, immaginando che i monti fossero popolati nella stessa misura della marina. Egli peró affermava di aver veduto, e con l'occhio suo esercitato, aver quasi contato, in una delle rade di Tahiti, un numero di uomini, atti a portare le armi, che poteva ascendere ad oltre diecimila.

Un dotto ufficiale della marina francese, il signor De Bovis, in un pregevole studio sullo stato della società tahitiana all'arrivo degli europei, dimostrò, che l'isola di Borabora, dell'arcipelago della Societá, con mille anime appena, nel 1852, doveva 
contarne diecimila ai tempi del suddetto viaģ̧io di Cook; e dedusse da questo fatto che, applicando un medesimo coefficiente a tutte le altre isole della Società, si perveniva, riguardo a Tahiti, al resultato ottenuto appunto dall'illustre marino.

Il missionario Wilson calcolava che la popolazione dei due istmi potesse essere, nel 1797, di sedicimila individui di oggni età e sesso, e ció in un periodo di rapidissimo decrescimento nella popolazione stessa. Altri missionari contemporanei dell'Wilson asserirono che, al loro giungere nell'isola, il numero degli abitanti era tale, che gl'indigeni, nella stagione delle pioggie, si affrettavano con le canoe a raccogliere la legna trascinata in mare dai torrenti, per poter far cuocere le vivande. Gli alberi del pane erano piu numerosi di adesso, eppure spesso un solo albero era proprietá di parecchie famiglie, e non di rado avvenivano liti, non giá per il possesso di un albero, ma per quello di uno dei suoi rami.

Tutti i vecchi indigeni che, anni sono, rammentavano ancora la venuta di Cook a Tahiti, concordavano nello ammettere, che durante le due generazioni precedenti, ed anche nei tempi della loro gioventú, le epidemie avevano menato strage fra la popolazione tahitiana.

Ora, questa disproporzione eccessiva che ha dovuto, in passato, esistere tra il numero degli abitanti e la superficie del suolo produttivo, non spiegherebbe forse, con qualche evidenza, il perchè della storica dissolutezza dei costumi locali che, ostacolo fra i maggiori alla conservazione e alla propagazione della specie, doveva, nella mente dei legislatori, o per intuito delle masse, gradatamente equilibrare il numero degli abitanti con lo spazio di terra concesso loro dalla natura?

Il censimento di Tahiti, fatto con cura, nel 1829, dai missionari francesi, aveva segnato la cifra di 8568 anime. Nel 1848, Tahiti e Moorea annoveravano 9454 anime, e, nel 1877, 7550; pertanto, diminuzione sensibile.

Tutte le popolazioni indigene delle isole oceaniche sembrano 
trovarsi sulla medesima via di decrescimento. Nel gruppo delle Gambier, ad esempio, ove, nei primi tempi della loro scoperta, i due sessi si uguagliavano nel numero, oggi le donne sono un terzo meno degli uomini.

Vancouver, quando scoprì l'isola Rapa nel 1791, ne calcolò a 1500 gli abitanti. Nel 1826, il missionario inglese Davis suppose che gl' indigeni di Rapa ascendessero a 2000; in pochi anni si ridussero a 500, ed attualmente non sono che 150 !

L'arcipelago di Tuamoti, incluse le isole Gambier, annovera una popolaziōne di 8000 anime; quello delle Marchesi di 10,000; e quello di Tubuai di 550. Sono tutte cifre approssimative.

Arduo sarebbe il rintracciare la causa recondita di codesto fenomeno perdurante della diminuzione della razza indigena, malgrado la scomparsa delle epidemie, la cessazione degli infanticidi, dei sacrifici umani ed altri costumi selvaggi.

L'apparizione dei bianchi, con il loro strascico di espedienti religiosi, di speculazioni commerciali e d'intenti politici, mutò molte delle usanze ${ }^{1}$ di questi polinesiani, senza migliorare l'ambito; anzi parrebbe che il mutamento peggiorasse l'indole intima dell' indigeno sostituendo una semplice e superficiale vernice alla rozza corteccia.

1 Il capitano Michele Molines, maltese, domiciliato a Papeete fin dal 1842, ci raccontava che al suo giungere nell'isola di Tahiti aveva trovato, fra varie usanze barocche, la seguente: Ogni anno la Corona convocava, in assemblea, i diversi Capi e notabili dell' isola, perchè formulassero e statuissero leggi. Due partiti si trovavano tosto in presenza (quello dei savi e quello dei turbolenti), i quali, intavolata un'ardente discussione, non tardavano a venire alle mani. In questo pugilato di legislatori, i turbolenti erano sempre vittoriosi, e poichè il baccano aveva avuto per origine la proposta di coltivare le terre, di fare reti per la pesca e di fabbricare buone case, il trionfo dava luogo ad una grande upaupà dei vincitori, che correvano ai monti, per condurvi vita selvaggia, finchè durava la stagione dei frutti. Codesta gente, quando scendeva alla marina, era ricevuta con manifestazioni chiassose di gioia dalle rispettive famiglie, che andavano loro incontro. La quiete da quel giorno si ristabiliva fra gl'isolani, ma il disordine risorgeva alla successiva annuale assemblea. 
L'indigeno si è assimilato dei bianchi quanto poteva convenire alla sua puerile vanitá, o a soddisfare la brutalità raffinata dei sensi. Dessi non lo hanno dominato; lo hanno stordito, inebriato, ed egli, illuso, ha perduto la sua entitá, per vagare, privo di guida, fuori dell'orbita assegnata alla sua razza dalla natura e precipitare, tosto o tardi, nell'abisso.

Non è ufficio nostro l'indagare se i bianchi vollero e seppero ricondurre o no questi grandi fanciulli dell'umanità su di una via corretta, ma esprimendo un nostro pensiero diremo che si sarebbe dovuto favorire sempre, non contrariare mai la natura. I complicati ingranaggi dei Governi coloniali europei non sono intesi dagl'indigeni, tanto meno poi se hanno impronta militare; quindi non è che il self-Governement ${ }^{1}$ che potrebbe, forse, ancora salvare la societá indigena, quando le autorità europee, chiamate a controllarlo, scevre di pregiudizi, avessero unicamente in mira di coltivare la mitezza dei costumi, di secondare la creazione della famiglia, l'amore al lavoro. Così talvolta si otterrebbero dei produttori e consumatori validi.

I primi europei che presero stanza a Tahiti furono i missionari wesleyani, ai quali fecero seguito i missionari francesi cattolici. Più tardi, col protettorato della Francia, equipaggi di navi baleniere, disertori, avventurieri vennero ad ingrossare il nucleo dei residenti bianchi che, per altro, la scoperta dell'oro in California ridusse tosto ad esigue proporzioni.

Il Governo francese, per rimediare alla scarsezza dei coloni bianchi, fece appello, nel 1868, agli agricoltori californiani perchè piu vicini, e cessata la febbre dell'oro, allettandoli col rimborso delle spese di viaggio. Molti accorsero, pochissimi rimasero.

Ultimamente, l'impianto di vari importanti stabilimenti agricoli attirò un po' d'immigrazione bianca che, superiore per qua-

1 Altrove dicemmo che il desiderio della Francia di conservare gli usi e costumi indigeni, appariva dall'attuale legislazione, ma questa non è ancora il self-Governement a cui alludiamo. 


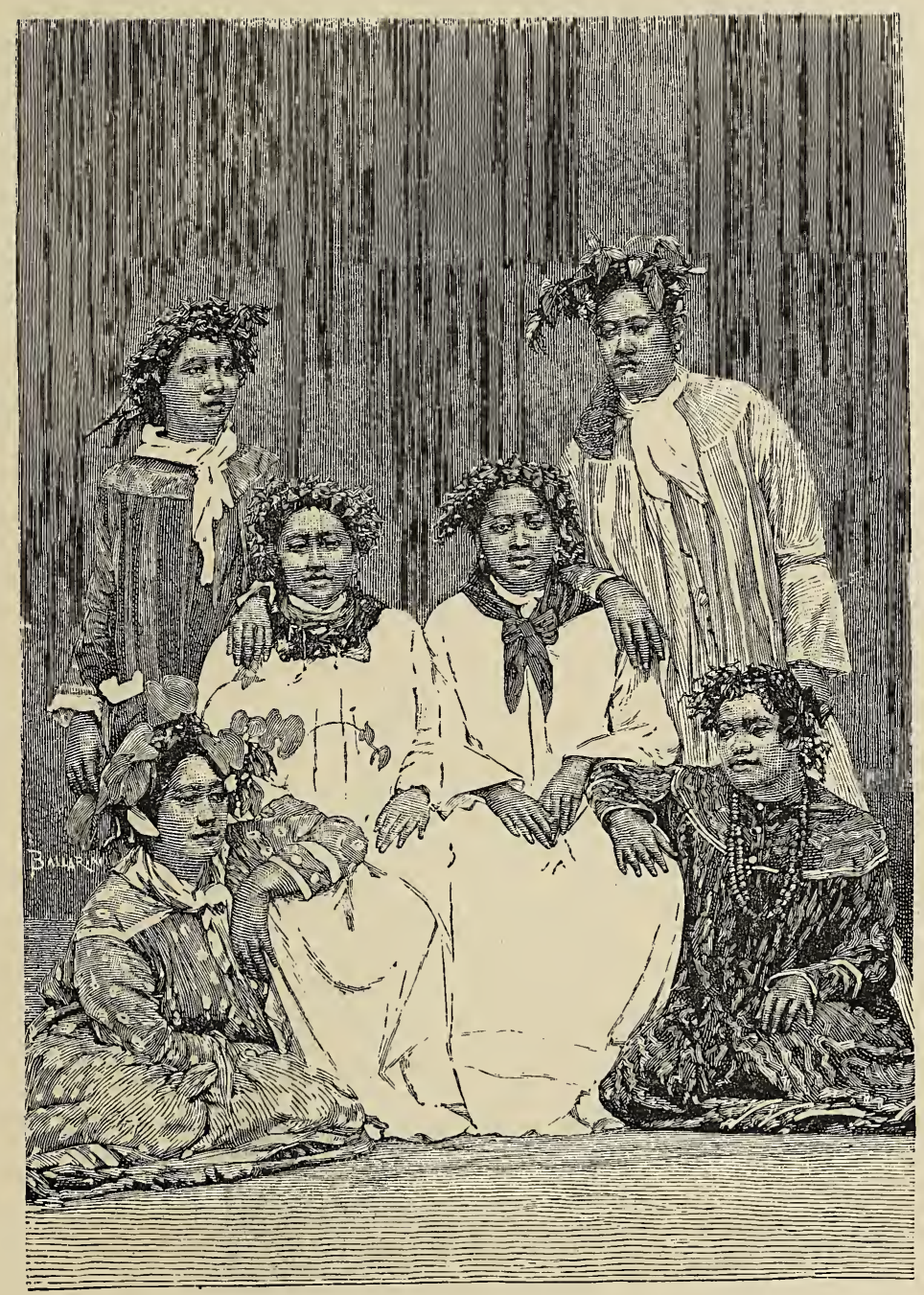

Costumi di Tahiti. 

lità di gran lunga alle precedenti, tende, quantunque lentamente, ad aumentare.

Nel 1848 gli europei e nord-americani domiciliati a Tahiti ed a Moorea erano in numero di 515, nel 1863 di 630, e nel 1877 di 1000, cifra che corrisponde all'attuale. ${ }^{1}$

II.

\section{Prodotti agricoli - Pesca.}

Altrove accenniamo che il suolo delle isole della Società è pietroso e duro sulla cima dei monti, che sugli altipiani intermedî si trovano sovente masse di argilla, ma che nelle vallate ed in riva al mare uno strato di terra assai spesso lo ricuopre rendendolo atto a tutte le produzioni tropicali. Ora, questo strato si estende per circa 25,000 ettari, sopra una superficie totale di 104,215 ettari, per Tahiti, e nell'isola di Moorea per 3500 almeno, sopra una superficie totale di 13,237 ettari. Nel dare vita ad una vegetazione rigogliosissima, sebbene poco variata, potrebbe far prosperare quella che proviene dall'importazione di altri paesi caldi, notevole per fecondità, ed assumere una certa importanza agricola.

Ma sia l'indolenza dei figli del paese, dipendente dai loro limitati bisogni, a cui provvede con generosità la natura, sia il piccolo numero d'intelligenti e doviziosi agricoltori bianchi, sia la difficoltá di ottenere immigranti lavoratori, e sieno infine le scarse comunicazioni con i grandi centri, fu ed è tuttavia ben meschino lo sviluppo della ricchezza agricola locale.

Nel 1865 si contavano appena 200 ettari di terreno coltivato:

${ }^{1}$ Non sono compresi gl'impiegati e loro famiglie e la guarnigione, che rappresentano in tutti gli Stabilimenti, complessivamente, 400 persone circa. 
cotone, canna da zucchero, caffè, ${ }^{1}$ vainiglia, tabacco. Tre anni dopo se ne contarono 2162 ; peró, questo aumento eccessivamente rapido dipese dalla fondazione di una Società Anglo-Franca, ${ }^{2}$ iniziatrice di vaste piantagioni di cotone e di caffè, con due milioni di franchi di capitale.

L'Amministrazione indigena, sino dal 1863, per incoraggiare i piccoli proprietari di terreni, particolarmente indigeni, aveva creato una Cassa agricola, che doveva prestare somme, dietro ipoteche, e pagare anticipatamente 1 franco e 75 centesimi per ogni chilogrammo di cotone greggio e non sgranato. La Cassa agricola dovera poi incaricarsi di fare sgranare il cotone, con l'impegno di rimborsare al proprietario la differenza, nel caso in cui il prezzo di vendita avesse dato un benefizio, deduzione fatta della spesa. Codesta provvida istituzione, che fiorisce tuttodi, è divenuta anche Cassa di risparmio per gli agricoltori.

Verso il 1879 le grandi piantagioni decaddero, per mancanza di braccia, nel momento precisamente in cui la qualità dei prodotti si trovava notevolmente migliorata.

L'allevamento del bestiame equino e bovino, che ha nesso sì stretto con l'agricoltura, non corrispose mai, nè a Tahiti nè a Moorea, alle aspettative della popolazione bianca. Cavalli e buoi macilenti, a cagione dei magri pascoli e dell'assenza d'intelligenti cure da parte degl'indigeni.

Pullulano i maiali, saporiti di carne, a Moorea, negli isolotti Tetiaroa, nell' isolotto Maitia, distante 60 miglia all' Est di Tahiti. Pochi gli ovini e poco il pollame.

Fra gli agricoltori europei primeggiano attualmente, a Tahiti, i missionari cattolici francesi, sotto la direzione di monsignor

${ }^{1}$ Per la coltivazione del caffè lo strato terroso fertile non ha sempre sufficiente profondità.

2 Diretta da un uomo abile ed energico, il signor Stewart, essa aveva vinte le difficoltà sempre grandi di un primo impianto, quando dissesti finanziari improvvisi la posero in liquidazione. 
Tepano, vescovo di Axieri. Coltivano specialmente la canna da zucchero e l'albero di cocco.

Per bontà di coltivazione l'isola Moorea supera, da qualche tempo, Tahiti, i suoi maggiori prodotti consistendo in cotone, vainiglia ed alberi di cocco.

È nell'isola Moorea che il signor Micheli ${ }^{1}$ organizzó una bellissima tenuta agricola, che gli valse un premio dal Governo locale, nel 1879, epoca nella quale essa comprendeva 5 ettari di granturco, 20,000 alberi di cocco, in pieno rendimento. L'insieme di quella tenuta conserva sempre un aspetto splendido.

Dicemmo sopra che verso il 1879 le vaste proprietà agricole tahitiane caddero in sofferenza, per mancanza di braccia; possiamo aggiungere che la loro odierna situazione economica è pressochè quella di allora.

Mancano le braccia! è l'esclamazione di tutti gl' industriali europei, che si recano in lontane contrade, apportandovi attivitá e capitali, per moltiplicare celeremente questi con l'aiuto di quella.

Ma i canachi sono troppo ricchi per adattarsi al basso ufficio di braccianti, dacchè il più povero è sempre in grado di supplire alle modeste necessità della sua serena esistenza, cogliendo pane (fei) al monte, e dedicando brevi istanti del giorno alla pesca. Dopo tutto, potrebbe il tahitiano aver ragione di rifiutarsi, senza un bisogno assoluto, a sacrificare la libertà, che gli è sì cara, l'indolenza, che lo contenta e non gli nuoce, per trasformarsi in un cieco istrumento di speculazione straniera. I benefizî che ritrarrà il suo paese da codesta speculazione, che deve essenzialmente giovare al bianco, egli non li comprende, abituato com'è all'imprevidenza, ed a non valutare che il frutto diretto ed immediato delle sue fatiche. ${ }^{2}$

Le braccia, se le si vogliono, fa mestieri pertanto cercarle al di fuori; ma dove?

1 Si parla del signor Micheli a pag. 22, «Gita a Moorea ».

2 Il Governo degli Stabilimenti, allorchè ha bisogno di braccia a Tahiti, invia una nave nell' arcipelago di Tubuai e di Cook per reclutare lavoranti. 
Tahiti e Moorea sono perduti in mezzo al Grande Oceano! Un mese e piu li separa dall'America e dall'Australia, due lunghi mesi dall'Europa; e nessun bianco, con la penuria di lavoratori, che si fa sentire ovunque, vorrà mai immigrare in luoghi così lontani dalla sua patria, e ne' quali, quando volesse retrocedere, si troverebbe di fronte a spese forse sproporzionate a' suoi mezzi, e certo aggravanti in sommo grado i sacrifizi gia fatti.

I soli immigranti agricoli, ai quali si possa volgere un'attenzione seria, sarebbero gli asiatici: i chinesi, che con la modicitá del salario, con la pertinacia nel lavoro, rispondono a' requisiti del buon funzionamento d'imprese basate sulla produzione a larghissima scala. Ma i chinesi non sono simpatici; comunque esigui i loro stipendi, dessi, sobrî all'eccesso, non tardano a fare una concorrenza pericolosa ad ogni e qualsiasi piccolo commerciante; e ciò che è peggio, codesti asiatici laboriosi, dall'aspetto ripugnante, sono oltremodo depravati. sicchè il loro contatto con gli indigeni sarebbe deleterio.

Una Commissione del Consiglio coloniale di Tahiti proponeva nel 1882 di reclutare lavoratori fra gli abitanti delle isole basse, situate all'Ovest ed al Nord di Tahiti, convinta che, ad onta dei difetti di tali indigeni selvaggi ed ancora antropofagi, l'agricoltura tahitiana avrebbe trovato il suo tornaconto nel valersi della loro opera.

Si affaccia qui alla mente una riflessione: colmata che sia la lácuna relativa alle braccia, avranno vita duratura le vaste piantagioni, qualora i mezzi di comunicazione fra Tahiti ed i grandi mercati mondiali rimangano quali sono: monchi, cioè, scarsi e lunghi?

Non si può perdere di vista, che i possedimenti francesi, nell'Oceania centrale, sono rannodati dalla vela, e soltanto dalla vela, ai continenti, e che il giro degli affari e dei grandi affari, oggi giorno, si compie sull'arena della concorrenza, col sussidio del telegrafo e del vapore. L'attività dei grandi produttori, laddove difettino i mezzi rapidi di comunicazione, non è che una leva priva di punto di appoggio. 
A nostro avviso, soltanto il taglio dell' istmo di Panamá varrà a sciogliere l' intricato problema.

L'Arcipelago di Tuamotí, il cui territorio occupa una superficie di 500,000 ettari, è composto, ad eccezione del gruppo delle Gambier, d'isole, o meglio di atolli di 4 a 500 metri di fascia, con un lagone interno, che ha 100 miglia di circuito a Raicoa, e 90 nell' isola Takarova.

Ad Anaa gl'indigeni, in relazioni frequenti con le isole della Società, v'importarono il cocco, che crebbe quasi senza coltura, e si propagò d'isola in isola fino all'estremo levante dell'Arcipelago. Il frutto divenne la base del nutrimento indigeno e rese possibile di allevare maiali e pollame.

Vicino ad alcuni villaggi gl' isolani fecero fossi di 4 a 5 metri di profonditá, sopra 6 a 7 di larghezza e 13 a 14 di lunghezza, e riempitili di terra vegetale, li convertirono in giardini nei quali coltivarono il taró, qualche canna da zucchero, il banano, l'ananas ed il tabacco.

L'agricoltura delle isole basse si limita, in conseguenza, all'albero del cocco, i cui prodotti nelle isole maggiori: Anaa, Takarova, Takapoto e Niau, danno luogo ad esportazioni. Poverissimo il suolo delle isole; le acque invece che ne bagnano le rive interne sono ricche: racchiudono nel loro seno la madreperla, alle attrattive della quale sono dovute le prime visite di navi mercantili nella Polinesia.

Sventuratamente, l'avidità del guadagno, stimolando in questi ultimi anni una pesca eccessiva, distrusse le piu belle ostriche perlifere. Il Governo francese, per opporsi allo impoverimento dei banchi di madreperla e salvare questo ramo di commercio di esportazione, emanava, nel 1881, un regolamento, secondo cui i banchi stessi debbono essere sorvegliati dall'autorita, la pesca fatta solamente nelle epoche dell'anno designate e sotto speciali condizioni, e la vendita effettuata a terra, ne' luoghi all'uopo autorizzati.

Le belle ostriche perlifere, le ostriche dette madri, si trovano solo nella sabbia, ed ove si agitano correnti; in alcune isole, contengono perle nella proporzione del $65 \%$. 
Col mare calmo e l'acqua trasparente un indigeno marangone lo sono tutti - può prendere fino a 40 conchiglie in un giorno, ma non ne raccoglie, in media, che 15 .

Parrà strano che si parli ancora di marangoni per la pesca delle ostriche perlifere, ma pure è così : l'uso dell'apparecchio da palombaro non incontrò mai favore presso i canachi, mentre, adoperato dai bianchi, sarebbe troppo costoso.

Il gruppo delle isole Gambier, con una superficie di 3 mila ettari, è circondato da una barriera di coralli, che crea un lagone perlifero, simile a quello delle isole basse. Il loro suolo è frastagliato da monti, i più alti dei quali raggiungono i 400 metri.

Piccole vallate nascono da quei monti; ed è fra esse e la spiaggia, che si trova il terreno coltivabile, nel quale allignano le piante più utili delle isole della Società, come l'albero del pane, del cocco, il pandano, ecc.

Si vuole che il suolo prossimo alla piccola città di Rikitea, capitale del gruppo, sia anche appropriato alla coltivazione del caffè e del cotone.

I montoni, i capretti si acclimatano facilmente alle Gambier; il coniglio ivi vive allo stato quasi domestico. I maiali, un tempo numerosi, furono pressochè tutti distrutti.

La costituzione geologica delie Marchesi ha molta analogia con quella delle isole della Società, e, poichè l'analogia sussiste anche circa le condizioni del clima, così la feracità delle terre e la natura dei loro prodotti si rassomigliano nei due arcipelaghi.

La superficie totale delle Marchesi è di 125,000 ettari, dei quali pochi sono coltivati, gl'indigeni non avendo superato ancora lo stadio selvaggio.

Il bestiame abbandonato a sé vi si è moltiplicato rapida mente, ed, esportato, provvede al consumo di Tahiti.

Le isole Tubuai e l'isola Rapa non hanno alcuna importanza agricola. In quest'ultima isola, la cui superficie è di 2000 ettari, la disposizione geologica del terreno esclude le grandi vallate; ed infatti non s'incontra che una successione di piccole valli, 
senza direzione determinata. La vegetazione vi è quindi povera; l'albero del cocco cresce sì, ma i suoi frutti non maturano; manca l'albero del pane; ed il purau, il miro ed altri grandi vegetali delle isole più settentrionali, vi rimangono allo stato di arbusto. La sola coltura alla quale possono dedicarsi gl'indigeni è quella del taró.

III.

Industrie. ${ }^{1}$

Se i prodotti naturali delle dette regioni sono esigui relativamente all'estensione del territorio al quale essi appartengono, le industrie che dai medesimi derivano, e sono le uniche, hanno confini ancora più ristretti.

ll copra è di tutte la maggiore; poi vengono: l'industria dell'olio di cocco, quella del cotone, dello zucchero, del rhum, dei cappelli di paglia, delle stoie e della birra. Le prime tre figurano nell'esportazione, le rimanenti sono pel consumo locale.

L'industria dei cappelli di paglia e delle stoie è tutta canaca ossia indigena; la esercitano donne, che adoperando paglia di pia, ${ }^{2}$ di fei, corteccia di pandano e di bambi, rivelano nell'esecuzione del lavoro un gusto artistico degno di essere incoraggiato.

L'industria del copra è parimente indigena, e lo fu quella dell'olio, finchè gli europei non ebbero introdotto a Tahiti macchine per estrarlo. A titolo di curiosità descriveremo il modo primitivo, con cui. i canachi ottenevano l'olio di cocco.

${ }^{1}$ Ci piace ricordare qui, per debito di giustizia, che la ricerca de' dati relativi al commercio ed alle industrie della nostra opera è dovuta all'intelligente cooperazione del Commissario di bordo Cav. A. Bonucei.

${ }^{2} \dot{\mathrm{E}}$ un tubero filamentoso. 
Le noci essendo giunte a maturità, gl' indigeni ne grattugiavano la polpa con una lamina di ferro dentata, che era fissa ad un cavalletto ${ }^{1}$ indi deponevano la grattonata in tronchi di cocco scavati a forma di canoa, ${ }^{1}$ e la tenevano così esposta per due o tre settimane al sole. Spirato quel termine, la grattonata si era fatta pastosa; gl'indigeni allora la premevano fortemente con le mani, e, cavatane una buona parte dell'olio che conteneva, sottoponevano il residuo all'azione di un torchio, composto di due solide travi, la superiore delle quali terminava con una lunga leva.

I canachi ottengono il copra asportando, con un coltello, la polpa della noce di cocco spaccata in due, e disseccandola al sole sopra telai sospesi dal suolo.

Per accelerare la disseccazione della polpa taluno ricorre adesso al forno, ma il copra in tal maniera ottenuto perde del suo valore sui mercati europei.

Siffatta operazione del copra fornisce una cospicua quantità di borra, ${ }^{2}$ che ha molto pregio industriale; ma che gl' indigeni trascurano; e meno che a Tahiti, essa va completamente trascurata.

Le industrie locali, in cui si adoperano macchine, sono esercitate da coloni bianchi, ed hanno sede a Tahiti.

IV.

Commercio.

Papeete, porto principale dell' isola di Tahiti, è l'emporio isolano, il centro di circolazione delle arterie economiche di tutti gli «Stabilimenti francesi dell' Oceania ». Papeete avvia

1 Vedi volume I, tavola XIX.

2 È l'involucro filamentoso della noce di cocco. 


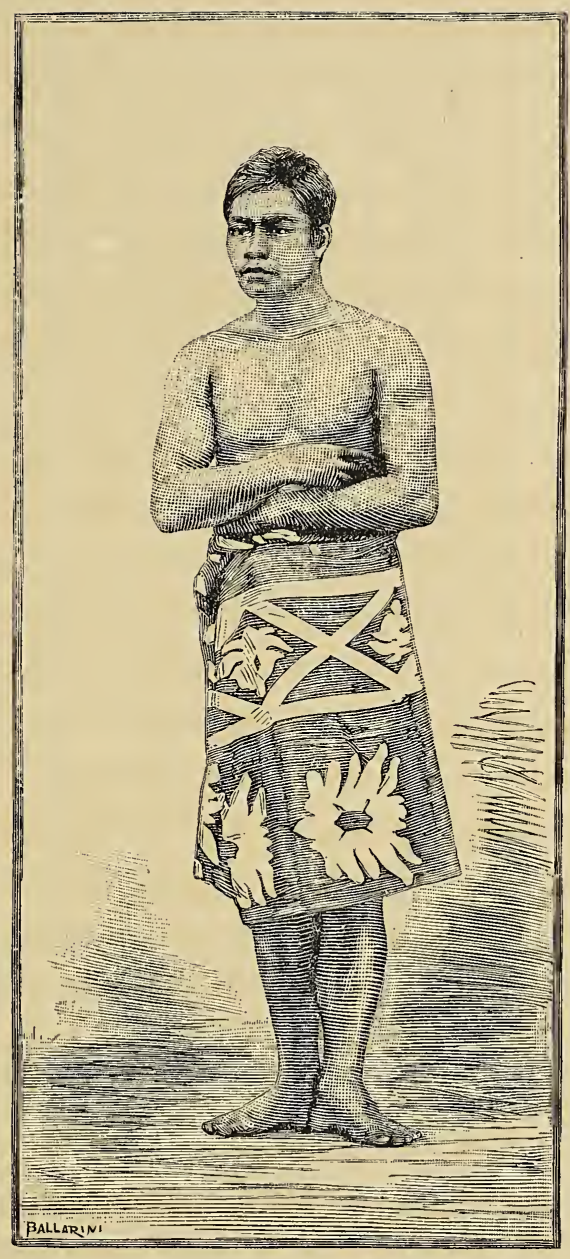

Canaca tahitiano. 

codeste arterie verso i continenti in mezzo ai quali giace e le richiama a sè vivificate dallo scambio dei prodotti.

Piccole navi del tonnellaggio massimo di 90, ed un centinaio d'imbarcazioni, raccolgono tutto attorno le esportazioni, e distribuiscono il bisognevole dell'importazione straniera. Un unico vaporino ${ }^{1}$ prende parte a quel movimento commerciale, di cui pertanto è anima la vela, con la sua poesia bensi, ma anche con la desolante realtà delle calme e dei venti contrari.

La vela non soltanto allaccia il porto di Papeete alle isole alte e basse, ma altresi alla metropoli, via S. Francisco di California, sebbene l'opportunità di una linea di navigazione a vapore fosse stata seriamente ventilata, nel 1881, ed il Consiglio coloniale avesse proposto a tale scopo un sussidio locale di 150 mila franchi.

I francesi, protezionisti sempre ed ovunque, riguardo alla loro marina mercantile, esclusero dall'esercizio del cabotaggio, nei loro possedimenti oceanici, le navi straniere, ed assegnarono ad esse, per il traffico dall'estero, soli sette porti: Papeete e Papeuriri (Tahiti), Opunohu (Moorea), Fakarova (Tuamotu), Mangareva (Gambier), Taio-hae (Marchese), Tubuai (Tubuai).

Nonostante cotali restrizioni, la navigazione di maggior conto, che è quella di lungo corso, si trova nelle mani di stranieri, e l'isola ha quasi tutte le sue relazioni commerciali con un paese estero: S. Francisco di California, da cui riceve i suoi approvvigionamenti di prima necessità.

Le numerose navi baleniere, ${ }^{2}$ che un tempo frequentavano Tahiti, erano pure straniere tutte.

Il gran commercio è fatto da un consorzio, che s'intitola:

${ }^{1}$ Il capitano inglese Tonne comperò nel 1879, in Australia, un vaporino per un servizio quindicinale tra Tahiti e le isole di Sottovento. Nel nostro soggiorno a Tahiti, in luglio 1883, non lo abbiamo veduto funzionare.

${ }^{2}$ La caccia insistente data un tempo alle balene le ha allontanate dalle acque tahitiane. 
Sociètè Commerciale de l'Océanie, e da case inglesi, ' nord-americane, tedesche e francesi. Il piccolo commercio trovasi ripartito tra francesi, indigeni e cinesi.

I residenti francesi si lagnano fortemente della concorrenza straniera, attribuendola all'indifferenza del commercio e dell'industria francesi per gli sbocchi che all'uno ed all'altra offrono i loro possedimenti di oltremare. Ritengono che per vincerla occorra creare grandi fattorie con capitali francesi, le quali importino dalla Francia direttamente e regolarmente nel loro raggio di azione i prodotti naturali e lavorati, ed esportino in Francia per la stessa via i prodotti indigeni che avranno coltivato.

Tahiti non possiede stabilimenti di credito, eccettone il Crédit-agricole, che non ha intenti bancari.

Se è vero che il commercio incipiente ha d'uopo di molta libertá, per mettere salde radici ed espandersi, si deve credere che quello di Tahiti sia esuberante di vita, imperocchè l'Amministrazione locale non gli risparmia vincoli, sotto forma di balzelli. Le mercanzie importate debbono pagare un diritto fisso del $12 \%$ sul valore indicato dalle fatture, con aumento dell' $8 \%$ per le spese accessorie; le bevande alcooliche pagano inoltre una fortissima sopratassa. Siffatto sistema di gravissime esazioni, che si estende sugli individui (testatico), sulle proprietá mobiliari, sull'ésercizio delle industrie e delle professioni, ecc. e sui cani, ? permette di saldare, quasi alla pari, ${ }^{3}$ il bilancio della colonia oceanica, che ammonta ad 1,193,970 franchi, somma i cui due terzi sono assorbiti dalla mentovata Amministrazione locale.

Negli anni 1880-81 il movimento commerciale marittimo medio di Tahiti fu, per le entrate, di 13,500 tonnellate, e per le

1 Sono quelle che dispongono di maggiori capitali.

${ }^{2}$ La tassa sui cani ha per iscopo la diminuzione del numero stragrande e pernicioso di codesti quadrupedi; ma i canachi preferiscono pagare anche forti tasse, anzichè rinunziare al fido animale.

3 Il Governo della metropoli accorda agli Stabilimenti oceanici 181,220 franchi sui bilanci dello Stato. 
uscite, di 15,500 tonnellate. Il valore medio dei carichi, nel primo caso, fu di 3,250,000 franchi, e nel secondo, di 4,340,000 franchi. Il valore complessivo degli articoli esportati, nel 1881, raggiunse la cifra di 2,938,769 franchi, ivi compresi franchi 340,000 per copra, cotone e fungus delle isole Marchesi. ${ }^{1}$

L'esportazione per l'Europa, nel 1882, ascendeva a circa 3 milioni di franchi, de' quali solo 100 mila appartenevano al commercio francese.

Nel 1882 le importazioni dall'Europa rappresentavano un valore di franchi 4,391,530, la Francia figurandovi per soli 662,267 franchi. ${ }^{2}$

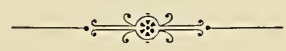

${ }^{1}$ I principali articoli esportati in quell'anno furono: madreperla in conchiglie, chilogrammi 492,420, franchi 989,888 - perle fine, franchi 20,658 Copra, chilogrammi 2,432,118, franchi 702,181 - cotone, chilogrammi 416,573, franchi 1,115,541 - semi di cotone, chilogrammi 872,970, franchi 21,821 vainiglia, chilogrammi 1,252, franchi 17,808 - aranci, n. 1,764,000, franchi 43,530 - cocchi, n. 271,573, franchi 26,642 - citrioli, n. 15,200, franchi 550 - banane (caschi) 300, franchi 150 .

2 I principali articoli importati nel 1882 furono: pitture preparate, franchi 830,229 - profumerie, droghe, ecc., franchi 233,074 - conterie, porcellane, specchi, cristalli, franchi 35,750 - tessuti, franchi 863,851 - carta, franchi 31,838 - materiale per navi, franchi 98,437 - mobili, franchi 30,330 - calzature, cappelli, ombrelli, chincaglierie, franchi 520,503. 



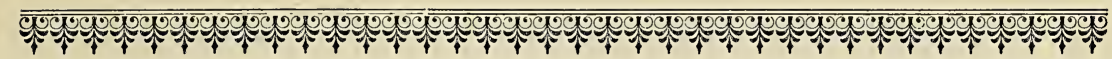

\section{CAPITOLO VI.}

G FOIOG IA DI TA HITI

I.

Cenni sulla costituzione fisica dell' isola.

Afferma il Darwin, nella narrazione del suo viaggio intorno al mondo, a bordo della Beagle, che in nessuna regione, neppure in quella delle Cordigliere, vide mai terre piu straordinariamente accidentate dell' isola di Tahiti. Aveva fatto una breve escursione nello interno, per la vallata di Tia-Auru, in cui scorre una delle principali riviere dell' isola.

I dati maggiori e più attendibili che si hanno sulla costituzione fisica di Tahiti si ripetono dal signor Garnier, ingegnere delle miniere, che nel 1866, insieme con il tenente di artiglieria di marina signor Mery, eseguì una esplorazione geologica nell'isola stessa. Il signor Garnier pubblicò sul giornale locale Messager de Tahiti il resultato della sua esplorazione in un'interessante memoria, da cui attingiamo i seguenti cenni.

${ }^{1}$ Consultare la carta annessa dell'arcipelago degli Stabilimenti oceanici francesi. 
La natura diversa della materia eruttiva e la sovrapposizione degli strati di lava recenti sugli antichi, dicono chiaramente come Tahiti debba la sua origine ad una serie di eruzioni vulcaniche, ch'ebbero luogo ad epoche varie. Queste eruzioni non furono continue, ed è facile il constatare che l'isola traversò lunghi periodi di quiete, nei quali il suolo doveva essere solcato da ragguardevoli corsi d'acqua. 亡 assai probabile che Tahiti appartenesse allora ad un vasto continente, ammantato di vegetazione, come lo attesterebbero gli alberi interi coperti di una colata di basalte, che si sono trovati nella vallata della Regina. Forse vivevano anche numerosi animali. Giunsero infine le eruzioni basaltiche, quelle de' trappi recenti e di tutte le rocce scoriacee delle quali l'isola è oggi quasi interamente formata. Per lungo tempo queste rocce in fusione colarono a guisa di torrenti, colmando dapprima i vani e le vallate inferiori formate dalle antiche eruzioni, e poi, condensati da successivo raffreddamento, sovrapponendosi a strati. Cessarono a loro volta le ultime eruzioni e l'isola presentò, a un dipresso, l'attuale conformazione fisica. Le conseguenti contrazioni determinarono screpolature in ogni dove, fra le quali penetrarono le acque, seguendo i declivi generali, ossia scendendo al mare. I letti primitivi si modificarono, si allargarono e formarono, in ultimo, le vallate dell'oggi. Molti secoli, certo, occorsero perchè si compiesse così immane lavoro. La superficie delle rocce vulcaniche si decompose, per produrre feracissimo terreno; e mazzi di piante e alberi incominciarono a spuntare qua e là. In mezzo a questa calma, i laboriosi zoofiti, che danno il corallo, si stabilirono in abbondanza sulle rive della costa di Ponente, ove i venti regnanti da S. E. non sollevano mai le onde per ritardare il loro lavoro. Dal giorno in cui s'innalzó, in tal modo, una barriera madreporica lungo la spiaggia, tutte le rocce e i detriti delle sommitä dell' isola, trascinati dalle acque verso la costa di Ponente, poterono accumularsi in un bacino tranquillo e sovrapporsi in maniera da formare la fascia di terra quasi orizzontale e fertilissima che 
si estende su tutta la costa di Ponente, al piede dei monti eruttivi. Questa fascia riposa, in molti punti, sopra gli stessi banchi madreporici, raggiungendo la sua massima larghezza all'apertura delle grandi vallate, e la minima nella vicinanza delle terre sporgenti.

Sulla costa di Levante, il mare, sempre agitato da' venti, non permise ai coralli di progredire cosi rapidamente, e si oppose pertanto alla formazione di una spiaggia estesa quanto quella della costa ridossata.

A Ponente di Papeete la fascia succitata è stretta e va diminuendo sino alla punta Taata, ${ }^{1}$ in cui è nulla. Vicino a questa punta vi è una cava di roccia composta di una pasta feldspatica grigia, spesso in decomposizione, che contiene piccoli cristalli di pirosseni augitici. Sulla punta stessa esiste una roccia rossa che, decomponendosi, dà un' argilla rossiccia commista a parti grigie. A mezzogiorno di Taata segue una serie di colline bagnate dal mare, le quali sono formate da una roccia pastosa, di color rossiccio, contenente cristalli di augite. La decomposizione di questa roccia fornisce argille molto rosse e molto ferruginose. Fra le colline ora dette ed il villaggio di Punaavia, ${ }^{2}$ al Sud di esse, corre una spiaggia sabbiosa sormontata da monti, che sebbene rivestiti di ricca vegetazione, presentano pendii ripidi, ripiegantisi, frequentemente, in forma di ferro di cavallo. A Punaavia vi è una riviera in cui rotolano rocce basaltiche, trappiche, trachitiche, scoreiformi, ecc., ecc. Colá la fascia di terreno alluvionale si allarga, e si scorgono corsi d'acqua più o meno vistosi, corrispondenti a vallate profonde, e foltamente boschive, le quali offrono generalmente un fenomeno particolare, quello dı aprirsi maggiormente nell' interno, e di essere, ad una certa distanza,

${ }^{1}$ La punta di Taata è situata a Ponente di Papeete, chilometri 5,5 di distanza.

2 Il villaggio Punaavia è al Sud della punta Taata, distandone chilometri $\%$. 
tagliate da semicerchi verticali, la cui altezza varia fra i 10 ed i 100 metri, formando cascate. Tali vallate, disposte a gradinate, assumono l'apparenza di anfiteatri.

A Maraa, ${ }^{1}$ punta S. O. di Tahiti, i monti arrivano al mare presentando uno spaccato interno, circolare e verticale di 150 metri di altezza, al cui piede sonvi due grotte. La piu notevole ha una profondità di 100 metri; è colma di acque alquanto profonde, alimentate da numerose infiltrazioni, cadenti a pioggia, dalle pareti della vôlta, sulla quale venne steso dalle acque un tappeto di calcare terroso roseo. Questa grotta, di forma semiconica, a larga apertura, ha per vertice il fondo della grotta stessa e per base l'entrata, che determina un effetto ottico, secondo il quale l'osservatore, stando presso all'entrata, si crede vicinissimo al fondo, mentre invece ne è lontano.

Ad Atimaono, ${ }^{2}$ località situata a Levante di Maraa, ed ove trovansi tutte le basi delle rocce dell' isola, la fascia di terra più volte citata spiega la sua maggiore ampiezza: tre chilometri circa.

Da Atimaono volgendo ancora a Levante, s'incontra la vallata di Vaihiria, ${ }^{3}$ rimarchevole per avere sui suoi fianchi una successione di semicerchi a pareti verticali, numerose cascate e parecchi coni di 100 e 200 metri di altezza, emergenti al centro, nelle alture.

È al disopra di questo gran circo che si trova il rinomato lago di Vaihiria, ${ }^{4}$ occupante la parte inferiore di un vasto imbuto, formato da alti monti ed aperto soltanto dal lato della vallata. Tutte le acque che cadono sui pendii ripidi di quel cono interno, si precipitano immediatamente nel lago, in numerose

1 Maraa dista da Punaavia chilometri 13.

2 Atimaono dista da Maraa chilometri 8 circa:

${ }^{3}$ La vallata di Vaihiria è distante da Atimaono chilometri 2 circa.

${ }^{4}$ Il lago di Vaihiria, situato a 430 metri al disopra del livello del mare, corona la vallata omonima. 


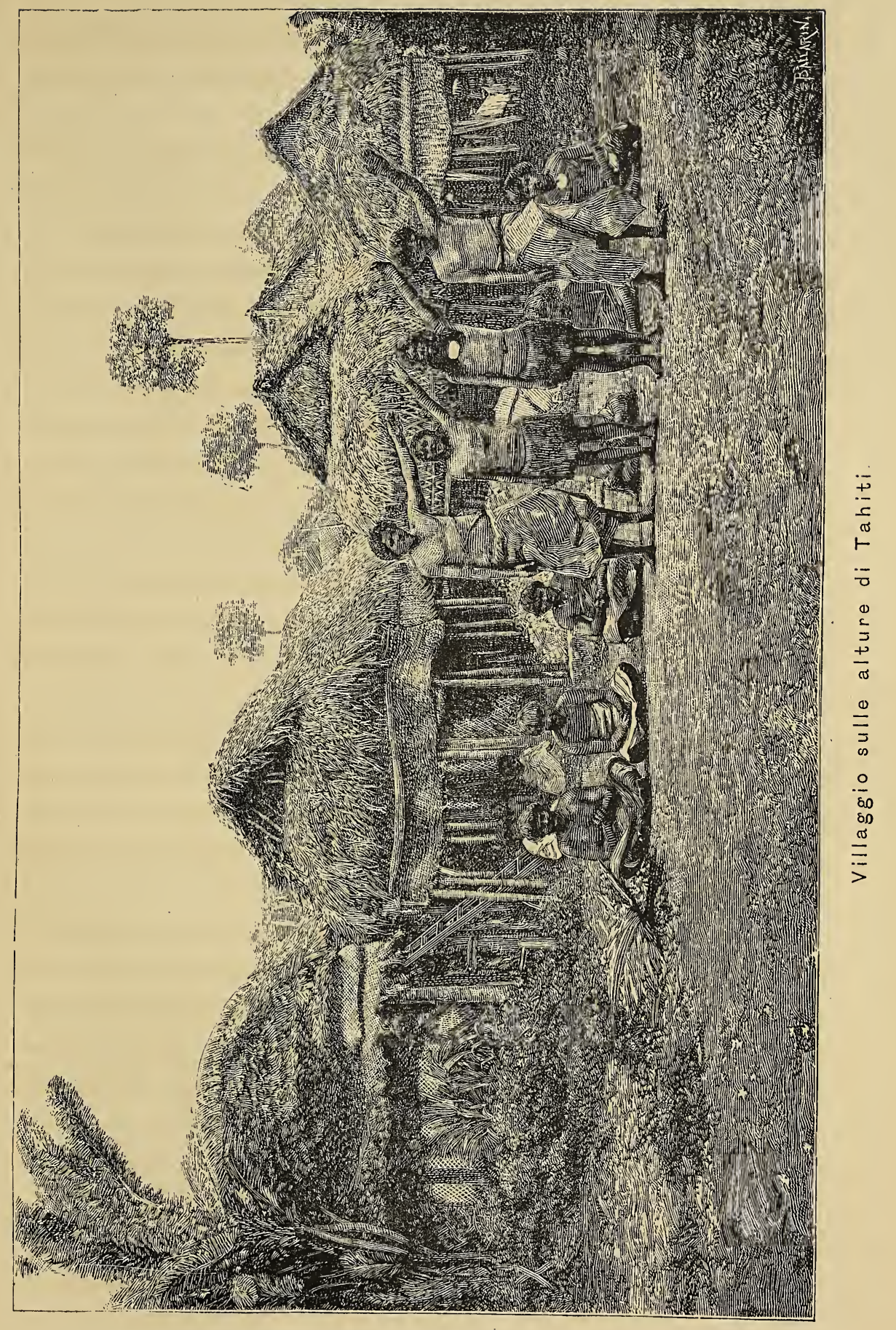



cascate, le cui linee bianche fanno contrasto con la cupa vegetazione di quei luoghi, sempre visitati da grosse nubi nere, che si muovono con lentezza sui fianchi e sulle vette dei monti.

Piove quasi sempre, al lago Vaihiria, la qual cosa si spiega col raffreddamento che le brezze del mare, sature piu o meno di umidità, provano subitamente nel giungere a tali altezze.

Gli orli del lago sono impraticabili e gl'indigeni per girarvi intorno debbono ricorrere al nuoto. Ha circa 500 metri di larghezza, variando in profondità, nelle vicinanze della riva, da 4 a 10 metri, mantenendosi altrove costantemente a 10 metri. Il fondo è fangoso.

Le rocce, che compongono i monti circostanti al lago, sono essenzialmente pirosseniche, della famiglia delle doleriti, e identiche a quelle di tutti i monti preaccennati. L'imbuto del lago non ha alcuna analogia col cratere di un vulcano, come alcuni credettero; l'assenza completa di lave, scorie, ceneri, ecc., nelle sue vicinanze, basterebbe per escludere subito ogni idea di cratere.

Ridiscendendo alla marina, si vedono scaturire a Levante di Vaihiria alcune sorgenti sufficientemente abbondanti per dare vita ad una riviera, sorgenti che gl'indigeni credono derivare dal mentovato lago.

A Papeari ${ }^{1}$ il suolo è esteso e fertile, con una vegetazione particolare che consta di folti gruppi di fiori e di bambú, ed una medesima composizione di rocce.

Ne' pressi dell' istmo di Tavarao vi sono argille provenienti dalla decomposizione di una roccia trappica, di forma sferoidale, solcata da piccoli filoni di un silicato magnesiaco biancastro tenero, traslucido ed untuoso. Nella roccia principale s'incontrano compenetrate anche rocce scoriacee.

L' istmo ha la larghezza massima di 2200 metri e la massima altezza di 14 metri dal pelo dell'acqua, nel punto in cui è situato il forte Tavarao.

1 Dista Papeari dalla vallata o fiume Vaihiria mezzo chilometro. 
$\mathrm{Al}$ Sud dell' istmo si trovano, vicino alla marina, altre argille abbondanti, che furon credute caolini, ma che hanno tutti i caratteri delle rocce basaltiche decomposte; alcune volte sembrano massi arrotondati. Procedendo innanzi verso Teahopo, che è a circa quattro chilometri dall'istmo, si è ad un tratto in presenza dei primi terreni sedimentari, formati da puddinghe, i cui strati sono diretti da N.E. a S. O., con l'inclinazione di $30^{\circ}$, e l'orientamento al N. O. che muta bruscamente, a qualche distanza, al S. E. Siffatte puddinghe, derivanti da frammenti basaltici e da quelle stesse rocce pirosseniche, che si vedono assai sovente verso l'interno dell'isola, contengono piccole cellule ripiene di concrezioni calcaree, aventi tutte le apparenze di polipi fossilizzati. Gli strati sedimentosi sono poco larghi e si protendono a mare; vengono in seguito, ed in abbondanza, i basalti. Da Teahopo in poi la nota fascia di terreno coltivabile diminuisce gradatamente, scomparendo del tutto nelle vicinanze della punta più meridionale dell'istmo. In questa nuova zona, i monti offrono una serie di picchi assai acuti, isolati, ovvero concatenati nei modi più bizzarri. Un po' prima di giungere alla punta meridionale dell'istmo, il mare si rompe violentemente contro la base dei monti. Il transito, per terra, riesce quivi quasi impossibile e, per mare, non manca di essere pericoloso. Risalendo al Nord non s' incontrano che coni di moderata altezza, generalmente arrotondati, i quali si presentano spesso, per serie sovrapposte ed appoggiate contro una catena, la cui linea di altitudine è parallela a quella che unirebbe le vette dei coni medesimi. Gole piu o meno elevate rannodano questi alla catena principale lasciando passare fra loro ruscelli che scaturiscono dalle montagne del secondo piano.

Chiuso così il circuito dell'istmo, e ritornando a Papeete, dall'Est, per primo punto rimarchevole si ha dinanzi uno spaccato scosceso detto One-Poto (sabbia corta) che giunge sino al mare, e lascia scorgere la costituzione esatta del suolo: sono letti sovrapposti di basalti, di doleriti, di ceneri, ecc., pressochè orizzontali, i quali accennano ad un certo tempo d'interruzione 
fra le varie colate. A Tiarei, ${ }^{1}$ che viene dopo, le rocce pirosseniche sono tenáci e granitoidee. Finalmente, nella vallata di $\mathrm{Pa}-$ penoo, ${ }^{2}$ ove trovansi le maggiori vallate e la piú importante riviera dell' isola, la costituzione geologica locale mostra alcune differenze notevoli con le rocce incontrate altrove; osservansi delle doleriti e de' basalti con zeoliti; delle rocce verdi, ancora indeterminate; delle sferoidee e calcareifere, contenenti geodi di carbonato di calce.

Da Papenoo ad Haapape ${ }^{3}$ nessuna particolarità.

Esiste ad Haapape, presso alla strada di cinta, una grotta curiosa, la cui struttura ha alcuni caratteri geologici salienti. Gl'indigeni non osano penetrare in essa, e fu il signor Garnier, primo fra gli europei, che la visitò.

Un anello ellittico circondato di colate di lava, di scorie e di trachite ne forma l'entrata, che è larga metri 1.40 ed alta 60 centimetri. Questa grotta singolare creata dalla natura, e dalla quale in altri tempi scorrevano costanti ruscelli di lava e di scorie, rassomiglia ad una galleria scavata nella roccia con straordinaria cura dalla mano dell'uomo. Le pareti interne, levigate come da un lungo sfregamento, sono in alcuni punti coperte di una vernice, che dinoterebbe un principio di fusione, ed in altri, mostrano screpolature prodotte dall'azione successiva del freddo e del caldo, alle quali furono sottoposte. Il suolo risale con un'inclinazione di $10^{\circ}$ circa ed è riempito di scorie, che si sono condensate sul luogo verso il fine dell'eruzione. Queste scorie di un giallo bruno sono arrotondate, accannellate, e presentano un'identità perfetta di colore e di forme con le scorie che escono dagli alti forni di coke, nei casi di cattivo funzionamento; in alcuni punti sono ricoperte di una crosta bianca, molto analoga all'opale. La direzione generale della grotta è N. $45^{\circ}$ E. e S. $45^{\circ}$ O. A 18 metri dall'ori-

1 Tiarei trovasi a 20 chilometri circa dalla Punta Venere.

2 Papenoo è circa 8 chilometri distante dalla Punta Venere.

3 Haapape è un villaggio vicino alla Punta Venere. 
fizio la larghezza è di metri 1.50 e l'altezza di metri 1.20 ; a 45 metri, l'altezza è di metri 2, la larghezza di metri 1.40. A 66 ed 82 metri, la grotta volge a sinistra; questo gomito ha trattenuto le scorie che ingombrano, in parte, il fondo. A 126 metri l'altezza è di metri 2.50, la larghezza metri 2 ; in ultimo, a metri 200 l'altezza non è che di 45 centimetri, la larghezza metri 1.20. Calore eccessivo, intollerabile.

Probabilmente la grotta comunica con un cratere, che si troverebbe ad una certa distanza, nell' interno. Però la presenza di un cratere in quei paraggi non fu mai segnalata; ma potrebb'essere che la sua apertura fosse stata ostruita dalla vegetazione, o, parzialmente, colmata da frane.

Il signor Garnier, nel punto in cui cessò d'inoltrarsi, cioè a 200 metri dall'entrata, riconobbe una piccola piramide, ch'egli ritenne dover essere un marae, ossia uno di quei monumenti che i tahitiani innalzavano ai loro Dei. ${ }^{1}$

La grotta ha la sua leggenda, che gli anziani di Haapape raccontano nei seguenti termini:

Molti anni or sono viveva nel villaggio di Haapape una vecchia nominata Nonà, da tutti tenuta in conto di una strega. Essa aveva una bella figlia, Hina, che un giovane, Monoihere, voleva sposare; ma non si sa il perchè, la vecchia Nonà si opponeva a questa unione. Che accadeva intanto? Quando Nonà, durante la notte, andava a pescare sulla scogliera, Hina si recava alla grotta, ove l'aspettava Monoihere, ed i giovani non si separavano che nel momento in cui la fiaccola, denunziando il ritorno della strega, si avvicinava alla spiaggia. Nonostante la notte, il luogo solitario del convegno e le tenebre fitte che regnavano nella grotta, Nonà tutto indovinó. Allora essa, come di consueto, prese la via della scogliera con la fiaccola in mano, e piantatala sulla punta di un corallo, se ne tornó difilato alla grotta, ove l'infelice Monoihere, ingannato dalla fiaccola, che brillava

1 Vedi capitolo II, Cenni storici, § I. 
sulle onde, aspettava la bella Hina. Il suo terrore fu grande nel riconoscere la strega; fuggì verso il fondo della caverna, e non ricomparve più. Da quell' epoca nessuno ha piu penetrato nella grotta, che prese il nome di Monoihere.

Fra Haapape e Papeete si trovano rocce vulcaniche, in alcuni casi, decomposte ed argille provenienti da queste decomposizioni.

Concluderemo che il massiccio dell' isola è costituito di una sola specie di rocce, di rocce basaltiche, escluso quindi il quarzo, il mercurio, il carbone, che si suppose dover esistere a Tahiti. ${ }^{1}$

Le più alte montagne dell' isola sono l'Aorai (metri 2064), l' Orohena (metri 2356) ed il Niri (metri 1324); quest'ultimo appartiene all' istmo di Tavarao.

Si aggiunga, in ultimo, che tutti coloro i quali studiarono la geologia degli arcipelaghi della vasta regione polinesiaca protetta dalla bandiera francese, concordano nell'affermare che l'analogia de' terreni di tutte le isole alte di tali arcipelaghi è così evidente da togliere ogni e qualsiasi dubbio intorno alla loro comune origine, la quale deve essere riferita a' medesimi sollevamenti, le rocce essendo identiche e disposte in un ordine simile.

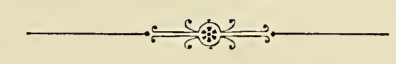

${ }^{1}$ Nella vallata di Papenoo, ai piedi di Pitohiti si trovarono alcune lave porfiriche. 



\section{CAPITOLO VII.}

\section{I.}

\section{I coralli.}

IL tacere dei coralli, avendo fatto menzione dell'aspetto delle terre tahitiane e della loro costituzione geologica, sarebbe omettere uno de' lineamenti più caratteristici della fisonomia fisica generale del paese.

I coralli sono parte integrante della geologia delle isole oceaniche, di cui, col proprio lavoro organico, completano la massa, se emerse, e ne rivelano l'esistenza, se nascoste nelle acque profonde del mare.

Ascesi agli estremi confini dei loro dominî, essi sorreggono il terreno vegetabile delle isole, che cinsero, per contribuire alla sua feracitá, ovvero collocandosi ad avanguardia, con un sistema di strategia perfetto, lo difendono dagli assalti soventi furiosi e sempre potenti delle onde marine.

Com’è noto, i coralli rappresentano agglomerazioni incalcolabili di polipi, che prendendo al mare porzione del carbonato di calce in esso contenuto, trovano nelle temperature intertropicali le migliori condizioni possibili di vita. 
Pertanto, l'Oceania, ambiente omogeneo a' polipi, offre saggi numerosi delle loro varie costruzioni corallifere: frangenti, barriere ed atolli.

I polipi invadono le coste ovunque, ma non si stabiliscono sulle rive composte di fango fino, e cessano di costruire dove grandi corsi d'acqua dolce intorbidiscono il mare, sicchè le isole, che per estensione e condizioni fisiche riuniscono siffatte circostanze, possono, grazie alla soluzione di continuità dell'opera polipaia, essere dotate di porti sicurissimi. Tahiti e Moorea, che si trovano in quel caso, dispongono di eccellenti rifugi nautici.

Parecchie ipotesi furono emesse per spiegare le differenti costruzioni dei polipi, e da valenti naturalisti, ma finora la questione è rimasta insoluta.

Verremo in aiuto alla memoria del lettore accennando alle ipotesi più attendibili.

Il Forster suppone che i polipi innalzino le loro abitazioni, gradatamente, da una considerevole profondità, stendendosi sempre più in larghezza, a misura che la costruzione tende ad elevarsi. Quando essa è vicina alla superficie delle acque, conchiglie, alghe, rottami di corallo, spesseggiano presso i contorni esterni, che sono i piì esposti all' urto del mare. Col tempo i contorni emergono, il centro, rimasto depresso, serve di letto al lagone, e sorge l'atollo.

Il Darwin tesse un' intera teoria:

I polipi, al dire dell'eminente naturalista, in balia delle correnti, fanno presa sui contorni di un'isola alta, le cui sponde si immergono con pendio dolce; crescono e determinano il polipaio frangente.

Il lato esterno di questo polipaio, esposto direttamente alle onde, riceve in gran copia nuovi polipi, e cresce più presto che il lato interno; inoltre, emerge maggiormente perchè riceve tutto ciò che il mare trasporta nella sua direzione. Avvenga uno di quei movimenti oscillatori della crosta terrestre, che si osservarono su vari punti del globo, movimenti lentissimi, ma apprez- 
zabili col tempo, e l'isola scenderà nel mare, ed i coralli che la cingono aumenteranno in altezza, per mantenersi al pelo delle acque, edificando nuovi piani sugli avanzi delle generazioni immerse. Dopo un certo altro tempo, il detto lato esterno sarà il solo visibile, lasciando fra esso e la terra ferma una depressione o fosso in cui si arresterà la produzione corallina, a cagione dei sedimenti dell'isola centrale, ed al largo apparirà il polipaiobarriera.

Ove continui il movimento di abbassamento, l' isola scomparirà del tutto, e si vedrá l'atollo col suo anello esteriore ed il suo lagone interno. ${ }^{1}$

Riguardo agli atolli taluno suppose che la loro forma dipendesse dallo avere i polipi scelto, per costrurre le loro abitazioni, gli orli di crateri sottomarini. Ragioni varie fecero rigettare questa supposizione. Generalmente i contorni degli atolli si allontanano dall' apparenza comune delle bocche ignivome e per le loro forme allungate, sghembe, e per le loro grandi proporzioni. Eppoi, nessun prodotto vulcanico fu mai trovato sugli atolli, la loro struttura essendo essenzialmente calcarea. D'altronde bisognerebbe ammettere, per la formazione di arcipelaghi estesi quanto quello di Tuamotù, ad esempio, un numero considerevole

${ }^{1}$ Per determinare l'altezza alla quale i coralli s'innalzano, in un certo periodo di tempo, nell'arcipelago della Società, il navigatore Wilkes (americano), stabilì nel 1839, sul litorale della baia Matarai (Tahiti), un punto fisso al quale riportò la sommità del banco Delfino situato a $1 / 2$ miglio nell'O. S. O. del faro di punta Venere, e ricoperto da 4 o 5 metri d'acqua, secondo lo stato della marea.

Nel 1860, l'Astrée, con la bandiera dell'ammiraglio francese Cloué, riferendosi ai lavori di Wilkes, ottenne una cifra, che avrebbe dovuto dare il cambiamento di altitudine del suddetto banco, ove non fosse scomparso uno dei segnali adoperati dal navigatore americano. Fece nuove osservazioni e determinò elementi di ricerca, che gli studiosi possono procurarsi dal Governo locale.

Tutte codeste circostanze pervennero a conoscenza della Caracciolo troppo tardi per farne suo pro. 
CAPITOLO VII.

di crateri agglomerati gli uni accanto agli altri, e giunti pressochè al medesimo livello.

Il Semper (di Wurtzbourg), fautore della teoria de' sollevamenti, riconobbe traccie evidenti di pressioni inferiori nelle vicinanze di atolli e di barriere, nei due arcipelaghi delle Filippine e di Pelew, che secondo la teoria di Darwin avrebbero dovuto deprimersi.

Il fatto citato dal Semper, agli occhi del capitano di vascello Jouan della marina francese, ${ }^{1}$ che in un interessante lavoro discute le varie ipotesi in argomento, rappresenterebbe semplici anomalie, le quali potrebbero ascriversi ad oscillazioni locali o ad alternazioni di innalzamento e di abbassamento, senza infirmare le leggi generali della teoria di Darwin, sola capace di dar ragione della formazione delle tre categorie polipaie, fasi diverse di un medesimo fenomeno.

Bisogna aggiungere, che mentre si sono osservati indizi evidenti di sollevamenti nelle isole alte, contornate di polipai-frangenti o prive di questi, come le isole Sandwich e le Marchesi, ed in alcune isole di origine madreporica, come le isole Loyalty, Tonga, Maatea, nessuno di cotali indizi fu mai scorto negli atolli e nelle isole alte, difese a polipai-barriere.

Un'obbiezione alla teoria di Darwin, che esclude l'esistenza dei polipi a grandi profonditá, la si ha nelle scoperte del Challenger, al Nord ed al Sud del Pacifico, di coralli viventi in fondi variabili tra 50 e 5220 metri, e dal Pourtalès, fra la Florida e Cuba, a 915 metri, con 100 atmosfere di pressione. Resta a sapere peró se le specie rinvenute sono proprio quelle stesse che edificano i banchi emergenti.

Darwin assegna alle zone di abbassamento del suolo, nell'Oceania, ragguardevoli estensioni in direzione dell'orientamento generale degli arcipelaghi, collocando fra tali zone, e parallelamente

1 Da quest'opera, che s'intitola Iles du Pacifique, abbiamo ricavato parechi dati che formano oggetto del presente capitolo. 
ad esse, fascie di sollevamento. La crosta terrestre sarebbe stata quindi sottoposta, nel vastissimo spazio occupato dal Grand'Oceano, ad enormi increspamenti, che avrebbero avuto luogo attorno ad assi diretti S.E. e N.O., cioè nel senso dell'orientamento suaccennato.

La immensa quantità d'isole, di cui è seminato quel mare, aveva fatto nascere l'idea, che le vette dei suoi monti piu elevati, fossero gli avanzi di un continente sprofondato, ma fu tosto compreso che, ammissibile l'idea stessa per le grandi terre, giacenti nell' $O$. e nel S. O. del Pacifico, nella struttura delle quali si osservạno le formazioni e gli elementi mineralogici dei continenti, non lo era piu per le piccole isole.

La costituzione delle isole del Pacifico centrale è delle piu semplici: rocce pirosseniche, che tutto indica, in modo chiaro, essere sôrte dal mare, per effetto di spinte energiche dal basso in alto.

Eminenti geologi opinano, che verso la fine dell'epoca terziaria, un gran continente oceanico ha dovuto sommergersi, e che le terre occidentali: Australia, Nuova Zelanda, Nuova Caledonia con le isole annesse, e Nuova Guinea, ne sieno le vestigia appariscenti. Il rimanente del Pacifico sarebbe stato un vastissimo deserto liquido, in mezzo al quale piu tardi, per azione vulcanica, emersero in direzioni parallele tutte le terre di cui si vedono oggi i polipai alterati dal denudamento. Codeste terre, dopo lunghissimo spazio di tempo, avrebbero subito gl'increspamenti ed i movimenti d'innalzamento e di abbassamento segnalati da Darwin, i quali danno all'Oceania l'attuale sua configurazione.

Torniamo un istante agli atolli per far cenno di una particolarità, che spesso richiama l'attenzione, e che consiste nei grossi blocchi calcarei deposti sul loro anello ed elevati 7 od 8 metri. Sono essi i punti estremi di un sollevamento calcare, formanti il nocciolo dell'isola, ovvero furono essi strappati dalla base del polipaio e colà gettati dalle violenti spinte del mare? Il coman- 
dante Jouan vede nella presenza di quei blocchi gli effetti di dislocazioni nella massa e di pressioni laterali cagionate da terremoti.

Faremo qui sosta, ed ammirando lo stupendo contrasto che offre la maestá del mare con la meschina natura del polipo, in questa lotta incessante dell'uno contro l'altro, ricorderemo le stupende parole del Darwin:

«... . sono miriadi di architetti che lavorano senza posa, giorno e notte, per legge di conservazione, dômando, coi loro corpi gelatinosi e languidi, la potenza meccanica delle onde, contro cui nè l'umana industria, nè la parte inanimata della natura potrebbero lottare con successo ». ${ }^{1}$

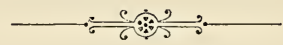

${ }^{1}$ L'andamento delle costruzioni corallifere, in virtù della simultaneità del lavoro, dalla periferia al centro, assicura all'intero sistema una perfetta uguaglianza d'innalzamento; sicchè il signor J. Dana ha potuto, nei suoi studi sui banchi coralliferi di Sandwich, giungere alla conclusione, che l'innalzamento annuo dell'edificio polipaio è di 3 millimetri. 


\section{CAPITOLO VIII.}

FAUNA F FLORA

I.

Visita all'atollo "Takumé g - Dragaggi nelle vicinanze di "Tuamotú

I' a tollo polinesiano, per chi lo guarda dal largo, crea illusioni ed ispira poesia; pare un'ampia canestra di fiori bianchi e cilestri con un gran mazzo verdeggiante nel mezzo: una corbeille degna delle nozze fra Peleo e Teti, la bella dea del mare.

Takumé, nell' arcipelago Tuamotú, fu il primo veduto dalla Caracciolo, e fu l'atollo scelto per gli escursionisti di bordo.

Difficile è lo sbarco, a cagione del mare che rompe e costringe a bagnarsi fino alle ginocchia, per poter discendere sopra un vasto piano orizzontale, largo 50 metri e ricoperto, a bassa marea, da 2 a 4 centimetri d'acqua.

Quel banco, che da lungi sembra ceruleo, apparisce sul luogo come un campo screziato di fiori variopinti con tutte le sfumature del rosso, del giallo e del violaceo. Sono coralli vivi: Astraee, Madrepore, Astrangie, Eliopore, ecc., vere colonie di polipi, fra gl' interstizi delle quali risiedono agglomerazioni immense di minori abitatori. Di questi si fece una piccola messe: quattro 
specie di Echini - una con le spicole a forma di clava; - una Asteria; delle Ofure. Vedevansi molte Tridacne sporgenti appena con valve semi aperte; due o tre specie di porcellette; ed una quantitá di Paguri a vivaci colori, che avevano stabilito la loro dimora entro a vecchi nicchi di Conus, Haliotis, ecc. Molte Morene di medie dimensioni, e grosse Aragoste (Palinurus marginatus).

Lasciata la riva, e dirigendo verso la laguna interna dell'atollo, si giunge dinanzi ad una vegetazione abbondante sì e rigogliosa, ma uniforme, e scarsa tanto di specie, che sarebbe difficile metterne insieme una cinquantina. Chiama l'attenzione una pianta frequentissima, dal portamento slanciato ed elegante: il Pandanus odoratissimus, le cui lunghe foglie servono a svariatissimi usi, come fu detto altrove. Essa vegeta a meraviglia in mezzo a sabbia e a pietre, e quando l'atollo è uscito dal seno del mare, è la prima a rivestirlo della sua verdura e ad imbalsamare l'aria coi suoi profumi.

Sui detriti e sul poco humus che deriva dal pandano vengono poi a stabilirsi altre piante: il tahenu (Tournefortia), l'Archyrautus lepidum; il Papyrus odoratus; varie felci; l'Hibiscus tiliaceus, la Thespesia populnea; il Calophyllum inophyllum; la Gardenia Tahitensis e parecchie altre che caratterizzano la vegetazione per così dire madreporica, specie tutte del litorale, facilmente trasportate dalle correnti su gli atolli. Dovunque poi si trova il Cocos Mucifera, la superba palma, che ebbe nome di Regina del Pacifico.

Colà, la povertá della flora è appena superata da quella della fauna terrestre, che solo è rappresentata da qualche uccello e da pochi insetti.

Nelle vicinanze dell' arcipelago di Tuamotí il bottino fatto, in parecchi giorni di calma, con il retino, riescì assai magro: qualche raro e piccolo Ctonoforo, una Rizofora piccola ma elegantissima, alcune Caprelle e qualche esemplare di un insettuccio marino (Halobates) comunissimo a Tahiti. S' incontrarono: uno 
squalo colossale ed un bellissimo pesce, lungo un metro e mezzo circa, dalle pinne pettorali di un colore azzurro metallico, che fu battezzato dai pescatori di bordo per Dorata della famiglia di quelle che in Sicilia chiamano Lampuca. Si prese un serpente del genere Hydrophis dai bellissimi colori, nero vellutato, nella parte dorsale del corpo, e giallo dorato, nel lato ventrale

II.

Cenni ge nerali sulla fauna terrestre, avifauna e fauna marina.

Le isole dell'Arcipelago della Società al pari di tutte le altre della Polinesia (eccettuate le grandi terre che occupano l'Ovest ed il S. O. del Pacifico), ripetono la loro origine da sollevamenti vulcanici; sono sparse lungi dai grandi continenti e peró la vita vegetale ed animale vi è necessariamente limitata ad un esiguo numero di specie. Ma è segnatamente nella fauna terrestre che si osserva questa scarsità.

Gl'indigeni di Tahiti prima della comparsa de' bianchi non conoscevano altri mammiferi che il porco, il cane ed un piccolo sorcio, e si puó affermare che i due primi e forse anche il terzo furono importati dai loro antenati quando vennero ad abitare queste isole. Il piccolo topo indigeno ora è quasi scomparso per opera del nostro grosso topo (Mus decumanus), il quale ha delle abitudini non meno cosmopolite dei bianchi che lo ospitano sui loro bastimenti.

L'avifauna è relativamente altrettanto povera, anche contando gli uccelli di mare e di spiaggia: Sterne, Fregate, Fetonti, Laridi, Aironi, ecc.; è gran cosa se si arriva a sommare ventidue o ventitre specie. Un Cuculo si trova a grandi distanze; una piccola Salangana nidifica sulle rive più scoscese, ed un gentile cantore, 
il Tatarè taitenà, fa sentire qualche volta i suoi trilli fra le macchie delle valli; la colomba Kurukuru, che si trova nella maggior parte delle isole della Polinesia, ed un grosso piccione, il Serrentius galeatus, rappresentano i colombidi. Altre volte era molto frequente un piccolo e grazioso pappagallo azzurro (Coryphilus dryas), ma i cacciatori l' hanno pressochè sterminato. Nessun uccello di rapina ha mai abitato queste isole; d'altronde vi morirebbe di fame.

A Moorea trovansi aironi ed alcune anitre (Anas superciliosa).

Gl'insetti, quantunque rappresentati da poche specie, grazie alle condizioni favorevoli del calore, dell'umidità e della potente vegetazione, si moltiplicano in grande numero d'individui e sono sempre più o meno nocivi.

Per ultimare anche con gli artropodi, aggiungeremo che una Scolopendra è l' unico animale velenoso in Tahiti, poichè non vi ha alcun serpente terrestre; l'erpetologia si trova ridotta a qualche lucertola e ad un geco.

Molto più ricca è la fauna marina, specialmente la costiera. Il pesce ha larga parte nella nutrizione de' polinesiani; la pesca si fa in generale di notte con fiocine e reti, e la rada di Papeete, quando è immersa nelle tenebre, si vede solcata da canoe con grandi fiammate di legno resinoso sulla prua, moventisi sull'acqua come fuochi fatui. I nativi usano anche stordire i pesci con mandorle di una mistacea, che cresce lungo il mare, la Barringtonia speciosa, e con legume della Tephrosia piscatoria. Abbondano i generi Serranus, Labrus, Scarus Plectropoma, Holocentrum, Sparus, gli Squammipenni; gli Scombridi, ecc. In questi mari sono pure frequenti i Tetrodon, le Murene, gli Squali, e gli Exoceti. Ad eccezione di certi Tetrodon non vi s'incontrano le specie nocive e velenose così frequenti nelle lagune interne di alcuni atolli di Tuamotú e sulle coste della Nuova Caledonia.

La maggior parte de' molluschi appartengono alla fauna indocinese. Le specie che s'incontrano nella Polinesia non sommano a più di 120 o 125, di cui alcune son notevoli per la mole che 
raggiungono; è da citarsi fra quest'ultime il Triton variegatum, che entra sovente in uso in una orchestra canaca, e la Meleagrina margaritifera piu frequente nelle isole basse di Tuamotú e sulle scogliere delle isole Gambier. La pesca di queste grandi ostriche, che formano la madreperla, e talvolta perle di notevole bellezza, fu altre volte sorgente di grossi guadagni.

Si è studiato il modo di crescere e di moltiplicarsi delle ostriche, e se ne son dedotte le regole, per rendere piu proficua quest' industria ora languente. Dopo un certo numero d'anni, da dieci a dodici, il mollusco resta pressochè stazionario nel suo sviluppo, depone ancora le uova, ma le sue valve deperiscono più o meno, perchè vengono traforate da altri animali; la bellezza e lo spessore della nacre varia secondo il fondo, dove sta deposto il mollusco; il fondo di sabbia fina è il più favorevole. Un carattere della malacologia di queste isole si è che le specie nude sono pochissimo numerose e che le conchiglie univalvi sono di gran lunga più comuni delle bivalvi.

La bizzarra ed irrequieta famiglia dei crostacei, oltre che da numerose specie de' principali generi di mole grande e media, è rappresentata da una quantità di piccoli Granchi e Palcemon, che abitano gli interstizi delle ramificazioni coralline; fra i Paguridi il Birgus latro, che può vivere molto a terra, si incontra comunemente a Tahiti come a Tuamotú.

La spiaggia sabbiosa di Papeete e delle insenate di Moorea era addirittura crivellata da innumerevoli buchi dove annidano dei grossi granchi tozzi e fulvi (genere Trapetia); essi sono la disperazione delle massaie, poichè con le loro chele formidabili mozzano sovente le zampe dei pulcini. Preferibili per la loro utilità sono i Gammarus e i Talitri, e gli eccellenti Palcemon, che si trovano in tutti i piccoli corsi d'acqua e figurano spesso nella cucina indigena ed europea.

Ma la parte piu interessante della fauna marittima di queste isole è senza dubbio quella degli animali inferiori.

Sulla scogliera, a Tahiti, si raccolsero alcune belle Oloturie 
ed altri Echinodermi, ed alcuni Anellidi. A Moorea, i dragaggi fatti, presso l'ancoraggio, alla profondità di 30 a 40 metri furono poco felici. Sarebbe stato meglio fare raccolte a bassa marea sugli scogli scoperti, o quasi, e dove il fondo, riconoscibile al colore ceruleo-latteo dell'acqua, non supera dai 7 agli 8 metri. All'interno ed all'esterno della scogliera, sopra un fondo di bianchi detriti, vegeta un mondo di Madrepore, di Gorgonidi, di Alcionidi dai colori più smaglianti.

III.

\section{Escursione botanica del Dottor Rhò.}

Il nostro dottor Rhò, fin da' primi giorni dell'arrivo a Tahiti della Caracciolo, conobbe l'egregio dottor Chassaniol, Capo del servizio sanitario, che volle aiutarlo nelle sue ricerche botaniche, piccola parte de' molti incarichi commessigli nella ripartizione generale de' nostri lavori.

Il collega gli esibì, in un suo giardinetto, alcune piante ornamentali indigene, fra cui primeggiava il tiarè (Gardenia Tahitensis), il fiore preferito dalle ragazze tahitiane, che lo coltivano presso le loro case, amando adornare e profumare con esso la chioma corvina. Pare sia stata importata, in tempi remotissimi, dalle isole madreporiche, dove si sviluppa meglio che nei terreni vulcanici. Gli indigeni, che sanno ciò, hanno cura di circondare la pianta con frammenti di corallo in modo da costituire un suolo appropriato alle sue attitudini. Nel giardino Chassaniol vi era un'altra pianta, evidentemente importata a Tahiti da' primi popoli che son venuti ad abitarla, la Broussonetia papyrifera, che si trova in molte isole della Polinesia. Questo piccolo albero, a Tahiti, non si moltiplica di per sè; non se ne incontrano che rari 
individui ed è destinato a sparire ben tosto, poichè la coltivazione ne è stata abbandonata dopochè le stoffe degli europei hanno sostituito quelle che gli indigeni confezionavano battendo la sua scorza. Anche piu interessante era una piperacea - il Piper methysticum - l'Ava degli indigeni, nota in tutta la Polinesia per la bevanda inebbriante e stupefacente che si trae dalle sue radici. Le ragazze masticano la radice, e ridottala a poltiglia, la diluiscono con l'acqua; pare che la reazione leggermente alcalina od i fermenti della saliva giovino a sviluppare le proprietà che rendono ricercata quella bevanda. ${ }^{1}$ Il dottor Rhò opina che, studiata la sua azione fisiologica, questa bevanda si troverebbe forse suscettibile di applicazioni terapeutiche, specialmente nei disturbi dipendenti da soverchia eccitazione nervosa ed abuso di forze intellettuali. Il Piper methysticum è divenuto raro a Tahiti dopo la proibizione di cui fu oggetto da parte de' missionari e dei Governi europei; non essendo più coltivato, come anticamente, ora si trova solo nelle sue stazioni naturali, in fondo alle grandi vallate, dove è rappresentato da una dozzina di varietà.

Invogliato il nostro dottore dal Chassaniol a fare qualche escursione botanica, scelse la cascata di Tantofoa, situata nella valle omonima a 4 ore da Papeete, ed accompagnato dal marinaro Zanardini e dalla guida indigena Tatamehe, partì di buon mattino il 22 luglio. Il dottor Chassaniol lo aveva, munito di una piccola monografia di un suo ex-collega, il dottor Nadeaud, intitolata: Enumération des plantes indigènes de Tahiti, libretto che quantunque fornisca solo la descrizione di poche fra le piante enumerate, pure nel dare anche il nome indigeno di ciascun vegetale, può servire a riconoscere facilmente le specie del'paese.

${ }^{1}$ La poltiglia è messa in un gran disco di legno, pronunziatamente concavo, e coperta d'acqua nella quantità determinata dalla consuetudine; indi è rimescolata con un fascio di erbe filamentose, a fine di raccogliere le parti lignee della poltiglia. Quando il liquido, in seguito all'operazione praticata col detto fascio d'erbe, che ogni tanto si attortiglia, è spoglio di tutte le parti lignee, la bevanda trovasi fatta, ed ha un colore latteo. 
I polinesiani in seguito al loro secolare isolamento, hanno dovuto necessariamente fissare la loro attenzione sulle piante, per cercare in esse di che vivere, vestirsi e guarirsi; tutte le fanerogame e quasi tutte le crittogame hanno il loro nome canaco, noto a tutti gli abitanti; e si direbbe quasi che essi possiedano vere nozioni di botanica, poichè sanno raggruppare anche le piante in famiglie abbastanza naturali.

Ma lasciamo che il Rhó stesso narri, con il suo buon umore abituale, la gita fatta a Tantofoa.

« Nei dintorni di Papeete İa zona di terra pianeggiante che circonda l'isola è alquanto larga ma è poco coltivata, ed è già gran cosa se si trova qualche piccolo spazio coperto di arbusti di cotone, che lottano infelicemente per l'esistenza col goyavier (Psidium piriferum). Quest'ultima pianta, importata cinquanta anni or sono dall'isola Norfolck (Australia), si è moltiplicata anche troppo soffocando in molti luoghi della spiaggia la vegetazione indigena, ed i suoi frutti dolci e buoni a far conserve sono magro compenso alle sue abitudini conquistatrici. Del resto, i tahitiani si danno poco pensiero dell'agricoltura; in fondo alle loro valli cresce spontaneamente quanto serve alla loro alimentazione; piantare cocchi costa poca fatica, e questi in poco tempo forniscono una buona vivanda alla loro mensa ed il pasto abituale ai loro porci. All'ombra de' cocchi, dappertutto i banani rallegrano il paesaggio con l'eleganza e la freschezza del loro fogliame vellutato. Una specie con molte varietá, la Musa paradisiaca, fu importata, senza dubbio, prima della venuta degli europei; i suoi frutti non portano i semi a completo sviluppo; si mangiano crudi e non sono certo inferiori ai famosi platanos de seda del Perú.

«La Musa fei è una specie veramente indigena e descritta, per la prima volta, da un botanico italiano, il Bertero. I suoi frutti molto più grosși si mangiano a diversi gradi di maturazione dopo essere stati cotti sotto la cenere calda; sono ricchi di fecola e molto nutritivi, ma verso il mese di dicembre racchiudono dei semi perfettamente sviluppati e suscettibili di ger- 
minare, che li rendono quasi immangiabili; a ragione gl'indigeni li designano con il nome di irii-ri, che significa piccoli ciottoli. «La mia guida Tatamehe mi diceva il nome di ogni pianta e da buon cicerone lo accompagnava di commenti e descrizioni, di cui io naturalmente non capivo gran cosa; egli camminava lesto, ma sfortunatamente perdeva molto tempo fermandosi ad informare ogni passante dell'importante sua missione e soddisfare ogni curiosità de' suoi connazionali sopra i due itariana ${ }^{\prime}$ (italiani). La relazione finiva sempre con una grassa risata a nostro riguardo, che se non fosse stata manifestamente ingenua, invece di esilararci, avrebbe finito per impermalirci. Per buona sorte, usciamo presto dalla strada maestra, che circonda l' isola, per rimontare la valle. Dopo un breve tratto di via polverosa lasciamo a sinistra una grande piantagione di cotone della famiglia Brander, d'origine scozzese e da lungo stabilita nell' isola, dove possiede le più grandi proprietà. La stradetta si perde presto nel bosco; la nostra guida ci precedeva nello stretto sentiero dondolandosi nel modo caratteristico di tutti i canachi, per l'abitudine acquistata nel camminare in angustissimi passi dov'è necessario mettere un piede innanzi all'altro sulla stessa linea, ma la sua corsa era rapida, ed assicuro che non tutti avrebbero potuto tenergli dietro, massime dove il cammino era piu ripido.

«Strada facendo Tatamehe continuava a darmi lezioni sulla flora tahitiana masticando quel po' di francese che sapeva. Non era piu la vegetazione madreporica della spiaggia; le specie comuni alle isole basse del Pacifico avevano sempre piu scarsi rappresentanti, ed egualmente erano scomparse o quasi le piante che seguono l'europeo nelle sue migrazioni e che giungono in qualche luogo a cambiare la fisionomia alla vegetazione indigena; lo stesso Goyavier, che sembra tutto aver invaso, cedeva il campo davanti all'abbondanza delle felci, le quali non gli permettono altro che stabilire piccole colonie nei punti piu visitati dagli

${ }^{1}$ Il tahitiano pronunzia $r$ I'l. 
abitanti. Eravamo in piena foresta polinesiana; peró fra quelle piante esotiche, a' nostri occhi, alcune ce n'erano che ci ricordavano le più tiepide regioni d'Italia. Gli agrumi importati da Cook si sono acclimatati a meraviglia e prosperano in tutte queste terre come in casa propria; lá fra i cespugli di felci incontravamo ad ogni passo gli aranci ed i limoni caduti e mescolati sovente col $v i$, delizioso frutto che ricorda il mango, ma possiede solo una leggera aggradevole ombra del suo sapore resinoso. Lo Spondias dulcis, che produce il vi, è uno de' più belli ed utili alberi dell' isola; de' suoi tronchi superbi erano costruite altre volte quelle doppie piroghe sulle quali si combattevano le battaglie sanguinose ricordate da' canti canachi.

«Da touriste poco esperto e frettoloso, io fermavo l'attenzione specialmente sulle piante più originali e più vistose; fra queste, frequenti erano alcune Panax, alcune Urticee, p. es. il Mali (Ficus tinctoria), il cui succo dà con le foglie della Cordia subcordata un color rosso preparato dagli indigeni fin da' tempi piu remoti; il Pua (Fragrea Berteriana), albero bellissimo, il cui nome ricorda il nostro Bertero, il Mape (Inocarpus edulis) dal tronco caratteristico diviso fino a parecchi metri dal suolo in lame profonde, e ben conosciuto pei suoi frutti e mandorle commestibili, ecc. Ma ciò che rendeva quella vegetazione affatto nuova per me era la quantità di Orchidee, e piú ancora la profusione di Felci d'ogni dimensione, che sostituivano la vegetazione erbacea e gli arbusti delle foreste, che finora avevo visto.

"C'erano de' piccoli Adianthum, Himenophyllum, delle Gleichenie, di cui sarebbero superbe le fioriere di un boudoir; e c'erano Pteris, Polypodium e Dicksonie arboree, che farebbero il vanto delle serre di un amatore. In quella rapida passeggiata ne raccolsi più di 40 specie.

«Il sentiero che noi seguivamo saliva e discendeva nella valle costeggiando la piccola riviera che scorre nel suo fondo; sulle sue sponde crescevano spontaneamente con altre Aroidee il Caladium esculentum ed il C. macrorhyza ossia il Tarò degli indi- 
geni, che dà una radice alimentare di cui si fa grande consumazione dai nativi.

"La vallata si faceva sempre più stretta e dovemmo guadare due volte il torrentello per risalire poi i fianchi del monte; anche là la nostra guida non pensó bene di rallentare il passo, e si fu con un sospiro di soddisfazione che udimmo finalmente dallo scabroso sentiero rumoreggiare la cascata vicina. Ancora un centinaio di rnetri, ed il bianco nastro d'argento ci si mostró cadente a precipizio, sopra un tappeto verdeggiante da un'altezza di quasi cento metri, in mezzo a mura basaltiche quasi nude e nere. Ci voleva proprio quel bello spettacolo per farci dimenticare che a causa di quel diavolo di Tatamehe eravamo stanchi e trafelati come cani a cui penzola la lingua fuori di bocca. Risalimmo sui fianchi della valle fino al disopra del salto; lassù c'è ancora il piccolo forte eretto dai francesi nel 1845, e dove si combattè l'ultima battaglia contro i tahitiani, che volevano scuotere il giogo del protettorato. Poco lungi, un parigino, stanco del mondo, si è fatto una casetta di legno, ed in quel romitaggio passa la sua vita. In altri tempi si sarebbe fatto frate, ma della sua tendenza alla vita claustrale questo signore deve aver conservato l'amore per le delizie gustative; infatti lassi coltiva con successo delle ottime fragole. Ci rincrebbe che il padrone fosse assente e più ancora che le fragole non fossero in grande quantità, per farcene un buon piatto. Facemmo il nostro pasto frugale all'aria aperta e fu allegro anche senz'avere il dessert onorato delle fragole: Levate le mense ebbi la soddisfazione di fumarmi una sigaretta sdraiato sulle felci e a 1000 metri sul solito livello, in mezzo all'Oceano Pacifico. Ma per mancanza di zolfanelli avrei dovuto far senza della sigaretta se la guida non ci avesse procurato in pochi minuti del fuoco confricando due pezzi di legno dolce detto Mao (Melochia ispida). Questa pianta cresce solo a piú di 800 metri, e sulla spiaggia è sempre sostituita dall'Hibiscus tiliaceus. Era la prima volta che vedevo usare questo sistema primitivo di fiammifero, e constatai, che la manovra ha bisogno di 
una certa pratica. Seguendo le spire del fumo guardavo le creste dei monti circostanti che s'innalzano a piu di 1500 metri col loro profilo bizzarro ricoperto ancora di vegetazione arborea: è la regione favorita di alcuni mirti (Metrosideros collina, ecc.) Assorto in quella beata contemplazione, già stavo per schiacciare un sonnellino quando dal cielo, coperto di fitte nubi, cominciò a cadere pioggia a catinelle. Non c'era da far altro che ritornare sui nostri passi, ma per una buona ora quella maledetta pioggia seguitò a seccarci e a bagnarci nello stesso tempo.

«In queste parti elevate dell'isola si accumulano tutto l'anno le nubi provenienti dalla condensazione dei vapori, di cui sono pregni i venti alisei. Si puó dire che non passa giorno senza pioggia; questa unitamente alle abbondanti rugiade notturne vi mantiene una costante umidità, la quale favorisce lo sviluppo prodigioso della vegetazione.

"Il lato meridionale ed orientale dell'isola, esposti maggiormente al vento di S. E., si trovano anche nelle parti più basse, nelle stesse condizioni delle parti centrali più elevate; la pioggia vi è frequente anche nella stagione secca, la temperatura più bassa che al lato N. dell' isola, ed i vegetali dell' interno discendono, per così dire, sulle sponde del mare. Queste condizioni non si trovano nel lato N. e N. O., donde l'aspetto nudo e languente delle prime colline, nei dintorni di Papeete».

IV.

Moorea - Maggiori particolari sulla flora polinesiana.

La parte dell' isola Moorea da noi visitata essendo esposta allo aliseo, presenta una vegetazione più rigogliosa che non il distretto della capitale e gli altri vicini.

Essa ha, se pure è possibile, una superficie più rotta ed acci- 
dentata di quella di Tahiti, è irta di picchi che paiono cincischiati artificialmente ed offre scene di selvaggia bellezza, in modo speciale dove le montagne cadono a picco sul mare. Infatti, una zona pianeggiante propriamente non esiste o per lo meno è ridotta a poca cosa nelle profonde insenate, a guisa di fiordi, da cui è frastagliata.

Vi si ritrovano, oltre ad altre piante già accennate, quella bella malvacea che è l'Hibiscus tiliaceus, che ombreggia con i suoi viali le case di Papeete; è il fau degli antichi, purau dei moderni indigeni, che ne utilizzano le foglie, i fiori, la scorza ed il legno. Lungo la spiaggia spiccavano, pel verde cupo delle foglie lunghe e sottili, annosi alberi di ati (Calophyllum inophyllum) che fornisce un legno ricercatissimo dagli europei per farne mobili, ed il Thespesia populnea il cui tronco dà lo stimato legno di rosa, l'albero sacro dei marae, dagl' indigeni chiamato miró; la Casuarina equisetifolia dalle fibre durissime, che danno al suo legno la consistenza e la durezza, per le quali ebbe il nome di bois de fer.

A Moorea, come a Tahiti, cresce l'albero del pane (Artocarpus incisa), l'uro o maiorè degli indigeni, per i quali forma parte importantissima dell'alimentazione. In entrambe codeste località i semi abortiscono costantemente ne' frutti e ciò con vantaggio della parte nutritiva, mentre nelle isole degli Amici lo stesso Artocarpus produce dei semi perfettamente sviluppati. ̇̀ dunque fuor di dubbio che quest'albero è straniero al suolo dell'arcipelago della Societá, dove se ne vede un gran numero, per la coltura di cui è oggetto. Si è giả accennato, parlando del banano, a quest'uso degli emigranti della Polinesia di portare con sè, partendo alla ricerca di nuove terre, le specie commestibili di prima necessità, per propagarle nelle isole dove si poteva non incontrarle. Anche ai nostri giorni si è stati testimoni di simili avventure. Non piu di vent'anni fa, una tribu intera delle isole Marchesi, vinta in guerra, per ubbidire ad un oracolo, s'imbarcò con armi e bagagli, dirigendo sopra un'isola ignota! 
A Moorea, a pochi passi dall'abitato, la vegetazione diventa subito selvaggia, e, per poco che si salga, s'incontrano, come nelle sommità più elevate di Tahiti, i tipi caratteristici che collegano la flora tahitiana a quella de' gruppi più lontani dell'Oceania.

In quella guisa che le isole Sandwich, la Nuova Zelanda, le isole degli Amici, l'orientale isola Pasqua (Rapa-Nui), ecc., poste sotto disparate latitudini, sono tutte abitate da una stessa razza, che parla dialetti di una stessa lingua, che beve il Kava, che riconosce la legge del tapiu, cosi tutte dividono con Tahiti le specie botaniche di certi generi quasi esclusivamente oceanici. Tali sono i generi: Angiopteris, Fitchia, Byronia, Cyathodes, Botryodendron, Caprosma, Astelia, Geniostoma, Santalum, Cyrtandra, Wickstroemia, Melicope, Evodia, Blackburnia, ecc.

Come si è fatta questa distribuzione di vegetali? Il dott. Hooker, così benemerito della botanica polinesiana, suppone che un gran continente si estendesse altravolta dal Chilì alla Nuova Olanda. I piccoli centri di vegetazione, che sono costituiti dai diversi gruppi di isole, e di cui i punti di riavvicinamento sono abbastanza caratteristici, troverebbero in tal guisa una spiegazione sufficiente. Ma contro l'opinione di Hooker sorgerebbe la costituzione geologica della Polinesia. Il problema non ha ancora avuto una soluzione completa; tuttavia, molti fatti che prima parevano inammissibili, come il trasporto di grani e semi per mezzo di venti e correnti, con una maggior conoscenza della meteorologia della Polinesia, ora si possono ragionevolmente spiegare.

L'Oceania tropicale ha dei vegetali che le son propri, ma una gran parte delle piante s'incontra in quasi tutta la distesa della zona intertropicale attorno al globo; ad esempio: l'Hibiscus tiliaceus, l'Urena lobata, il Calophyllum inophyllum, l'Abrus precatorius, la Guilandina bonduc, il Cocos nucifera, l'Ipomaea Pes caprae, parecchie Terminalia, Cordia, Barringtonia, ecc. Questi vegetali, che abbiamo citato, hanno dei semi che galleggiano sull'acqua e conservano per lunghissimo tempo la facolta di germinare. 
Qualche pianta dell'Oceania ha origine americana, altre provengono dall'Africa, ma esse sono in piccolo numero, comparate a quelle di origine asiatica. I viaggiatori che hanno percorso il Grande Oceano Pacifico, dall'arcipelago malese fino alle isole più vicine all'America, l'anno notato l'uniformità della vegetazione sulle terre sparse su quest'immenso spazio. Secondo alcuni, la Nuova Guinea formerebbe il centro geografico di una vegetazione particolare, alla quale fu attribuito il nome di flora litorale oceanica. Da questo centro le piante si sarebbero sparse nel resto dell'Oceania, con diversa sorte, in conformità dei luoghi e della loro posizione geografica; sicchè a Tahiti si avrebbero solo 508 piante conosciute e sull'isola Rapa-Nui a mala pena una ventina.

Restano le specie propriamente polinesiane, a riguardo delle quali le recenti esplorazioni della Nuova Guinea potranno forse illuminarci. Del resto, non ripugna ai moderni naturalisti la possibilità della formazione di nuove specie in date località e sotto speciali circostanze, e che poi possano propagarsi ad altre terre che presentino le stesse condizioni. Chè se queste specie non emigrano dalla loro patria di origine, vuol dire che anche in località vicine non hanno incontrato l'ambiente loro favorevole. Per citare un esempio locale, la Lobelia arborea, ${ }^{1}$ curiosa per la fenditura longitudinale della sua corolla, cresce nell'isola di Raiatea ed invano si cerca nella vicina Tahiti. ${ }^{2}$

${ }^{1}$ Il Comandante della goletta da guerra francese Orohena, che si occupa di botanica, favorì un esemplare di questo fiore.

2 Il dottor Nadeaud, già citato, dice che la cifra delle specie da lui riconosciute indigene di Tahiti si eleva a 508, ripartite come segue:

Piante cellulari: Epatiche, 42 - Muschi, 49.

Piante vascolari: Monocotiledonee, 196 - Dicotiledonee, 221. - Totale 508.

Le alghe ed i funghi molto numerosi; i licheni non vi sono compresi.

Una delle particolarità della flora tahitiana è l'estrema abbondanza delle felci. Se si aggiungono loro le Licopodiacee, si eleva a 12\%, contro 290 Fanerogame, cioè un po' meno della metà. Nella classe delle felci, 15 famiglie sono rappresentate: Gleicheniacee, 1 - Cyateacee, 2 - Hymenofiee, 14 - 


\section{V. \\ Il botanico Bertero.}

Esaurito il nostro tema, pagheremo un mesto tributo di ossequio alla memoria del compianto botanico italiano più sopra mentovato, accennando brevemente alla vita ed alla prematura fine di lui, che lo collocano fra' nobili martiri della scienza.

Il Bertero, membro dell'Accademia delle Scienze di Torino, nacque in quella città. Avviato nella medicina, prese gusto alla botanica. Compiuti i suoi studi, parti, giovane ancora, per le Indie occidentali, rimanendovi parecchi anni; egli ne portó in Europa ricche collezioni, che lo fecero conoscere al mondo scientifico, ed il suo nome prese posto fra quelli dei migliori scienziati viaggiatori. Dopo un breve soggiorno in Francia e nella sua città natale, volle visitare altri paesi e continuare le sue raccolte, stimolato più dalla passione che sentiva per la botanica e dal desiderio di essere utile alla scienza, che dall'amore della gloria.

Davalliee, 11 - Pteridee, 12 - Lomariee, 6 - Aspleniee, 12 - Polipodiee, 18 - Grammitidee, 8 - Acrostichee, 7 - Schizeacee, 2 - Marattiee, 3 - Ophioglossee, 3 - Licopodiacee, 11. - Totale $12 \%$.

Fra le 79 famiglie di Fanerogame, 33 sono rappresentate da una sola specie, 7 famiglie hanno almeno 10 specie; queste sono:

Graminacee, 18 - Ciperacee, 12 - Orchidee, 19 - Urticacee, 10 - Rubiacee, 22 - Euforbiacee, 16 - Papilionacee, $1 \%$.

La flora tahitiana è quasi tutta a foglie persistenti. Durante la stagione secca (maggio-settembre) qualche specie indigena si spoglia completamente delle sue foglie, per non riprenderle che alle prime pioggie dell'inverno, in ottobre. Fra queste: l'Erythrina indica, le Spondias dulcis, i Gyrocarpus asiaticus. 
Il paese che preferì dapprima per le sue ricerche fu il Chilì, dove arrivò nel 1828. Là, mettendosi tosto all'opera con lo zelo infaticabile che lo animava, esplorò una gran parte della Repubblica; nel 1830, condottovi dal Capitano P. King sull'Adventure di S. M. Britannica, visitó l'isola di Juan Fernandez e vi scoprì alcune specie nuove, fra cui una felce arborea, la Dicksonia berteroana. Di ritorno da quest'esplorazione, conobbe in Valparaiso un negoziante belga, il signor: L. Moerenhout, stabilito da poco tempo a Tahiti. Questi gli parló delle isole polinesiache, della loro ricca vegetazione, e sapendo che aveva l'intenzione di fare qualche nuovo viaggio, gli offrì passaggio per Tahiti sopra una sua goletta. Per disgrazia sua e della scienza egli accettò. Giunse a Papeete il 3 novembre dello stesso anno, invaso da ardente entusiasmo all'aspetto dei tesori che il nuovo soggiorno forniva alla sua scienza. Incantato dei luoghi, contento delle scoperte che vi faceva, benchè vi riconoscesse più ricchezza che varietà di vegetazione, cominciò le sue ricerche con grande ardore e formò collezioni immense fino al 9 aprile 1831, in cui lasciò Tahiti, tanto a cagione dei torbidi che vi regnavano allora, quanto per la notizia a lui pervenuta dello scoppio della rivoluzione francese. Moerenhout lo consigliava ad attendere qualche mese ancora l'arrivo di un bastimento più grande e più comodo, ma non riusci a dissuaderlo, e giunto il giorno fissato, essi si diedero l'ultimo addio. La goletta toccò Raiatea, donde il Bertero scrisse un'ultima volta al suo amico, parlando di piante che gli erano state portate e che egli non conosceva; esprimeva il suo rimpianto di non avere a sua disposizione libri ed oggetti, ed aggiungeva che sarebbe rimasto nell'isola se li avesse avuti. Il bastimento lasció Raiatea alla metà d'aprile e non se ne intese più parlare. Moerenhout visitò poi varie isole, le sole su cui il Bertero avrebbe potuto salvarsi, ma inutilmente. Non resta alcun dubbio pertanto che egli si sia perduto in alto mare insieme con tutte le cose sue.

Durante il soggiorno di Tahiti il Bertero non si occupava solamente di piante. Come medico vi rese de' grandi servigi ed 
era sempre pronto a dare i soccorsi della sua arte a chiunque si recasse a sollecitarli; ma s'egli era pieno di desiderio d'essere utile, non amava le molestie inutili. D'una vivacità estrema, gli capitò spesso di lasciarsi trasportare dallo sdegno, il che dava luogo à scene singolari ed eccentriche, in cui spiccava l'umorismo del nostro botanico. ${ }^{1}$

Per l'ingegno, per gli studi e per le opere, se non fosse stato rapito alla Patria ed alla scienza dall'infido elemento, egli avrebbe occupato degnamente il suo posto accanto agli Hooker, ai Forster, ai Leeman ed altri, che hanno illustrato la flora della Polinesia. Il fato non gli permise di giungere alla meta, ma non per questo il Bertero dev'essere dimenticato dagli italiani. 


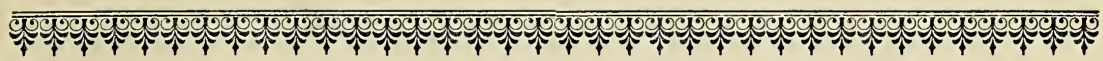

\section{CAPITOLO IX.}

I.

\section{Clima - Igiene.}

LE forze vulcaniche, che impressero un carattere cosi bizzarramente accidentato alla superficie dell' isola di Tahiti ed elevarono fino a considerevole altezza i materiali eruttati, contribuirono a rendere piu variato il clima di questo paese.

Il centro dell' isola è occupato da alte montagne, dove s'accumulano le nubi, e riceve pioggia in maggior copia che il litorale; la temperatura vi è molto al disotto di quelle del clima marino delle spiaggie, e le brusche variazioni della temperatura del giorno e della notte danno luogo a rugiade d'un'abbondanza estrema, le quali vi mantengono un'umidità costante, che favorisce lo sviluppo prodigioso della vegetazione. È a credere che i reumi di tutte le forme si manifesterebbero in un modo non meno straordinario se questa parte dell'isola fosse abitata.

La penisola di Taiarapu o Tavarao ed il lato di Hitiaa sono esposte al vento di S. E., dagl'indigeni chiamato Maraamu, vento freddo ed impetuoso, che apporta sovente la pioggia, anche nella stagione secca, allorquando Papeete e i suoi dintorni godono varî periodi di bel tempo. 
Le uniche osservazioni meteorologiche che si facciano nell' isola sono quelle dell'ospedale di Papeete. Le osservazioni del 1882, annesse al presente capitolo, ${ }^{1}$ sono estratte dalle note giornaliere del dottor Pleigneaux, addetto a quello stabilimento sanitario. La pressione barometrica varia fra millimetri 756 e millimetri 766 ; le oscillazioni diurne non superano mai i 5 o 6 millimetri e le loro indicazioni non hanno un gran valore poichè queste località non sono esposte a grandi uragani.

Quanto alla temperatura si puó asserire che Tahiti gode di una perpetua estate, quantunque i calori non vi sieno mai veramente eccessivi. La temperatura minima di $18^{\circ} \mathrm{C}^{\circ}$ venne osservata in agosto ed il maximum di $33^{\circ}$ si ebbe in alcuni giorni di febbraio e marzo. Le medie mensuali oscillano fra i 24 ed i 27 gradi.

Il clima caldo è rinfrescato dai venti alisei, che, come si sa, in queste regioni sono sempre costanti. I vapori di cui sono carichi questi venti vengono condensati dalle alte montagne dove piove abbondantemente tutto l'anno, mentre talvolta sulle rive del mare cadono solo poche goccie. Il versante meridionale dell' isola, per la stessa ragione, come già si è detto, è più piovoso del settentrionale.

Vi è, cionondimeno, una stagione umida (dicembre-marzo) caratterizzata da pioggie torrenziali, ed una stagione secca, quantunque, come si vede dai dati pluviometrici annessi, questo alternarsi non abbia limiti ben definiti.

La città di Papeete ed i suoi dintorni per la loro speciale topografia godono di un clima piu asciutto, che qualunque altro punto dell'isola, ed i venti dominanti non spirano ivi da S. E. sibbene da O., da N. E. e da E. N. E., ma specialmente da N.E. Infatti l'aliseo, sormontato il gruppo orografico più elevato, noto sotto il nome di diadema, passa al di sopra della città senza farvisi sentire e non vi giunge che riflesso dall'isola di Moorea, situata

1 Vedi tabella a pagina 118. 
di fronte alla rada. È evidente che in tutto questo giro il vento ha dovuto spogliarsi di una gran parte della sua umidità.

Riassumendo, il clima è buonissimo e sano, per quanto può esserlo un clima tropicale, e gli europei vi si trovano bene, tuttochè a lungo andare vi perdano alquanto della loro energia, sia fisica che morale.

Lo stato civile, ottimamente organizzato a Tahiti, fornisce per il 1882 il seguente specchietto:

\begin{tabular}{|c|c|c|c|c|c|c|c|c|c|c|}
\hline \multirow[b]{2}{*}{1882} & \multicolumn{5}{|c|}{ Nascite } & \multirow{2}{*}{ 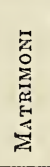 } & \multicolumn{4}{|c|}{ MoRTI } \\
\hline & 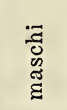 & 莺 & 谧 & 节 & 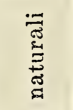 & & $\begin{array}{l}\stackrel{\Xi}{\Xi} \\
\stackrel{\Xi}{g}\end{array}$ & 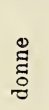 & 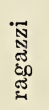 & $\begin{array}{l}\text { 我 } \\
\stackrel{4}{:} \\
\stackrel{0}{0}\end{array}$ \\
\hline $1^{\circ}$ trimestre. . & 38 & 31 & 69 & 43 & 26 & 15 & 26 & 21 & 11 & 58 \\
\hline id. & 40 & 35 & 75 & 51 & 24 & 13 & 36 & 29 & 20 & 85 \\
\hline id. & 35 & 43 & 78 & 57 & 21 & 13 & 25 & 28 & 20 & 73 \\
\hline id. & 48 & 34 & 82 & 48 & 34 & 17 & 38 & 20 & 17 & 75 \\
\hline Totali. . . & 161 & 143 & 304 & 199 & 105 & 58 & 125 & 98 & 68 & 291 \\
\hline
\end{tabular}

La mortalità non si può dire grande; essa è però stata accresciuta qualche anno addietro, da epidemie vaiolose, che fecero grandi stragi fra gl'indigeni (1854). Le altre malattie proprie dei tropici ben raramente si fanno sentire a Tahiti, ad eccezione della dissenteria che qualche volta ha decimato le popolazioni di qualche distretto, ove generalmente rimase limitata. Il colèra e la febbre gialla vi sono affatto sconosciuti.

Molti indigeni sono affetti da elefantiasi (fefe), che li deforma orribilmente; malattie pure frequenti sono il lupus (il piu sovente non exedens), la lebbra e le malattie squamose in genere.

Le malattie che riconoscono un'eziologia reumatica sono per cosi dire all'ordine del giorno; i reumatismi articolari cronici e deformanti diventano i fedeli compagni dei vecchi, i quali ne soffrono orribilmente. Ma la malattia che fa il maggior numero di vittime fra la popolazione canaca è la tisi polmonare; facili come 
sono gl'indigeni a procacciarsi ogni genere d'affezioni degli organi respiratorì e specialmente dei bronchi, per l'incuria insita alla loro indole fanciullesca, trasgrediscono anche le più semplici norme igieniche, e così niente di piu frequente che il vedere queste bronchiti degenerare in bronco-alveoliti con tutte le loro conseguenze.

I canachi sono comunemente scettici in fatto di medicina; usano solo grandi frizioni ed empiastri vegetali in quantita. Le loro medichesse fanno uso nell'emoptoe delle decozioni di frutta acerbe dello Spondias dulcis (vi degl' indigeni, pomme citerne dei francesi) che ad imperfetta maturazione sono ricche di acido tannico e di una sostanza resinosa analoga alla trementina; fino dai tempi piu antichi sono conosciute ed applicate le proprieti purgative di una convolvolacea Ipomea Turpethum, e di altre specie affini, comprese tutte col nome di Taurihau. Di ospedale non ne vogliono sapere, e quello di Papeete, aperto a tutti, non accoglie mai altri che europei. D'altronde gl'indigeni, quando sono ammalati, per fatalismo o per stoica rassegnazione o per incosciente insensibilità, accettano impassibilmente la loro sorte; sovente accade di vedere uno di questi colossi dal torso erculeo farsi irriconoscibile, in pochi mesi, ed aspettare tranquillamente l'ultim'ora.

Vi ha chi assevera che le malattie dei canachi, e specialmente la tisi, si sono rese più esiziali dacchè essi hanno adottato il nostro costume, di coprirsi tutto il corpo. Qui, dove le pioggie sono così frequenti, e la facilità con cui si espongono gli abitanti è così nota, l'asserzione parrebbe verosimile; ma si osserva lo stesso andamento nelle malattie fra gl'indigeni di certi distretti, che non hanno accettato i nostri vestiti, o che li portano solo nei dì di festa.

Le epidemie sono probabilmente la causa che ha contribuito maggiormente a spopolare queste belle isole, ma esse si ridussero sempre a qualche invasione limitata di dissenteria e, più raramente ancora, di vaiolo.

Dalla sifilide gl'indigeni guariscono con grande facilità; e 
per assicurazione dei medici del luogo è più rara che in Europa od America, nè mai vi si hanno ad osservare i terribili fenomeni terziari di questa malattia.

Quantunque tutti i polinesiani abbiano una marcata tendenza ad abusare delle bevande spiritose, l'ava (kava degli altri arcipelaghi), d'uso universale prima della venuta degli europei, non è da credere fosse meno dannosa delle nostre bevande alcooliche. Su queśto punto si è molto esagerato riguardo agl'indigeni. Noi che ci siamo trovati in giorni di festa a Papeete, non abbiamo mai veduto un canaco scandalosamente ubbriaco; ed il confronto che si poteva fare, era tutto a svantaggio degli europei.

Tutti i tahitiani in buono stato di salute si presentano vigorosi e ben nudriti, e le loro condizioni materiali e per certi punti anco le morali sono' superiori a quelle delle classi povere nostre.

Le valli fecondissime producono quasi spontaneamente anche più del bisogno i loro alimenti vegetali; il consumo della carne è anche proporzionatamente più grande che presso di noi. Mercè questo benessere materiale, l'istruzione si è sparsa facilmente; tutti sanno leggere e scrivere, e la mancanza dell'imperiosa necessità del lavoro per vivere, ha fatto colá, ciò che non farà mai in Italia la legge sulla istruzione obbligatoria.

I delitti d'ogni specie e segnatamente di sangue, che nel giardino d'Europa, das land wo die citronen bluen, prosperano talune volte non meno degli aranci e dei limoni, a Tahiti sono quasi sconosciuti. In un paese così felice si direbbe che le leggi economiche di Malthus, non dovrebbero mai trovare un'applicazione. Qual'è dunque la ragione per cui questa razza in breve volgere d'anni si trovò ridotta al disotto alla decima parte? 
II.

\section{Conclusione.}

Abbiamo cercato fin qui di ritrarre alcuni de'principali profili dell'economia di questa regione oceanica, che ha per cardine Tahiti, mostrando nudamente un mondo conosciuto, in generale, per il suo manto di aneddoti piccanti e di racconti favolosi; ora dobbiamo riassumerci e conchiudere.

La popolazione indigena dei possedimenti francesi nell'Oceania - circa 40,000 anime - tende ogni giorno più a sparire, mentre i bianchi che verranno a sostituirla nel paese disertato, salubre e ricco, saranno troppo pochi, attesa la distanza dal vivaio, per isvolgerne tutte le risorse e fruirne opportunamente.

È dunque un vuoto che si presenterà, tosto o tardi, e che non si potrá colmare!

Questo vuoto la Francia non lo previde, quando si eresse arbitra dei destini delle terre oceaniche, l'intimo di lei pensiero non essendo quello di beneficare il buon popolo polinesiano, nè l'altro di fondare una vera colonia agricolo-commerciale europea.

Lo spirito di rivalità non ancora assopito delle conquiste britanniche sui mari, ispirò, ad avviso nostro, gli atti compiuti dall'ammiraglio Dupetit Thouars a Tahiti ed alle Marchesi nel 1842; e quindi l'obbiettivo - eminentemente francese - del possesso oceanico dovè essere politico-militare. Ma sorsero le perplessità, chi sa? anche i timori, per cui Tahiti, anzichè divenire un baluardo di guerra, una base di operazioni bellicose, in difetto di criteri ponderati e d'indirizzo prestabilito, si trasformó in una semplice tappa di sollazzo per i marinai delle flotte francesi del Pacifico.

Codesta trasformazione nel giovare all'erario nazionale, che 
sarebbe stato costretto a spese ingenti per fortificare Tahiti, non indebolì la potenza militare navale francese, e tolse alla Francia la grave preoccupazione di dovere, in un momento dato, difendere seriamente possedimenti, che, lontanissimi dalla madre patria, sono per essa di un interesse economico molto problematico.

Tagliato che sia l' istmo di Panamá i detti possedimenti acquisteranno indubbiamente importanza maggiore uscendo dall'attuale languidezza, collocati come si troveranno sul corso della massima arteria commerciale, che rannoderà direttamente l'Europa e gli Stati Uniti all'Australia. Però, nel contempo, emergerà un inevitabile antagonismo tra Tahiti e le Marchesi. L'arco di circolo massimo taglia Tai-hae - eccellente porto delle Marchesi e di facile accesso, - ed abbrevia le distanze; Papeete, porto di Tahiti, fuori del circolo, allunga invece il cammino, obbliga le navi ad attraversare l' insidioso arcipelago di Tuamotì e non è sempre in ottime condizioni di sicurezza nautica. La sede del Governo coloniale verrà necessariamente trasferita a Nuka-hiva - capitale delle Marchesi - ove si risolveranno le vertenze che il movimento della navigazione farà nascere, e Tahiti dovrà cederle il primato.

Siffatto avvenimento, il quale renderà meno oppressivo il contatto europeo con gl'indigeni tahitiani, sarà egli una ragione di conservazione, e pertanto di salvezza dell'elemento indigeno? Vorremmo che ciò fosse.

Qualunque abbia potuto essere il motivo che determinò la Francia a porre piede sovrano nell' Oceano Pacifico, gli amanti imparziali del progresso umano debbono esserle grati di avere, con la presenza della sua bandiera, impedito che territori isolati e lontani dalle grandi comunitá civili potessero impunemente prestarsi a diventare il covo di avventurieri indegni e di pirati. 
Tabella delle Osservazioni meteorologiche

fatte all'ospedale di Papeete nel 1882.

\begin{tabular}{|c|c|c|c|c|c|c|c|c|}
\hline \multirow{2}{*}{ DATA } & \multicolumn{2}{|c|}{$\begin{array}{c}\text { PRESSIONE } \\
\text { BAROMETRICA }\end{array}$} & \multicolumn{5}{|c|}{ Temperatura } & \multirow{2}{*}{$\begin{array}{c}\text { VentI } \\
\text { D O MINANT I }\end{array}$} \\
\hline & $\begin{array}{l}\text { mas- } \\
\text { sima }\end{array}$ & $\underset{\text { nimi- }}{\mathrm{mi}}$ & mattino & sera & $\begin{array}{c}\text { media } \\
\text { mas- } \\
\text { sima }\end{array}$ & $\begin{array}{c}\text { media } \\
\text { mi- } \\
\text { nima }\end{array}$ & Pioggia & \\
\hline 1882 & & & $\mathrm{C}^{\mathrm{O}}$ & $\mathrm{C}^{\circ}$ & $\mathrm{C}^{\circ}$ & $\mathrm{C}^{\mathrm{o}}$ & & \\
\hline 29 dicembre, 4 genn. & 764.2 & 760 & 23.6 & 32.0 & 27 & 24 & 0.00195 & E. N. E. N. \\
\hline 5 all'll detto .... & 764.2 & 756 & 24 & 31 & 29 & 27 & 0.00104 & N. N.E. N. O. \\
\hline 12 al 18 detto..... & 764.2 & 759 & 24 & 31 & 27.4 & 26.8 & 000053 & N. N.E. N.O. \\
\hline 19 al 25 detto. & 761.2 & 757 & 24 & 31 & 27.6 & 27 & 0.00058 & N. E. E. \\
\hline 26 detto al $1^{\circ}$ febbr.. & 761.0 & 759 & 24 & 31.2 & 28 & 26 & 0.00115 & N. E. E. \\
\hline 2 all's febbraio ... & 762.0 & 759.2 & 24 & 32 & 27.4 & 25 & 0.00165 & N.E. E. \\
\hline 9 al 15 detto. . & 763.1 & 760.1 & 24 & 32 & 23 & 27.2 & 0.00065 & N.E. E. \\
\hline 16 al 22 detto. & 765.2 & 760.1 & 24 & 33 & 27.6 & 27.2 & 0.00010 & N. O. E. \\
\hline 23 detto al $1^{\circ}$ marzo. & 766.1 & 759.2 & 24 & 32 & 27.6 & 27.2 & - & E. N.E. \\
\hline 2 all's marzo . . . & 763.0 & 761.0 & 24 & 32 & 27.6 & 27.2 & - & N.E. E. \\
\hline 9 al 15 detto. . & 765.0 & 762.1 & 24 & 33 & 27.4 & 2.5 .4 & 0.00059 & E. N. E. \\
\hline 16 al 22 detto. & 765.0 & 762 & 24 & 32 & 27.4 & 27.2 & 0.00035 & E. N. E. \\
\hline 23 al 29 detto. & 765.1 & 759.1 & 21 & 32.4 & 28.1 & 27.2 & 0.00030 & N.E. E. \\
\hline 30 detto al 5 aprile & 765.0 & 760.1 & 23.4 & 32 & 27.4 & 27.1 & 0.00010 & O. N.E. \\
\hline 6 al 12 aprile... & 765.1 & 759.1 & 24 & 32 & 23 & 27 & 0.00010 & O. N.E. \\
\hline 13 al 19 detto. . . . . & 765 & 759 & 23 & 32.1 & 27.6 & 27.2 & 0.00085 & E. N. E. \\
\hline 20 al 26 detto... & 763 & 760 & 23 & 32 & 27.6 & 27.1 & 0.00010 & E. N.E. \\
\hline 27 detto al 3 maggio. & 763.1 & 762.1 & 24 & 30.2 & 27.4 & 27.1 & 0.00040 & E. N.E. \\
\hline 4 al 10 maggio.. & 7612 & 760.1 & 23 & 30.1 & 20.5 & $26 . \bar{i}$ & 0.00055 & N. N. E. \\
\hline 11 al 17 detto.... : & 764.1 & 760.1 & 22.1 & $30 \mathrm{l}$ & 27.1 & 26.2 & 0.00005 & S. E. O. \\
\hline 18 al 24 detto. . & 763.2 & 762 & 23 & 29.2 & 26.2 & 25.1 & 0.00005 & N. O. O. S.E. \\
\hline 25 al 31 detto. . . . & 763.1 & 761.1 & 23 & 30 & 25.2 & 25.1 & - & E. N.O. O. \\
\hline $1^{\circ}$ al 7 giugno. . . & 763.1 & 762.1 & 20 & 29.2 & 24.4 & 24 & - & O. S. E. \\
\hline 8 al 14 detto. . . & 763.1 & 760 & 23 & 28.1 & 24.6 & 24.1 & 0.00023 & E. N. \\
\hline 15 al 21 detto. . & 763.1 & 760 & 24 & 28 & 25.2 & 25 & 0.00095 & N. O. N.E. \\
\hline 22 al 28 detto. . & 761 & 762.1 & 24 & 28.2 & $2 \% 1$ & 25.1 & 0.00005 & E. N. E. \\
\hline 29 detto al 5 luglio & 762.1 & 760.1 & 24. & 28.1 & 27.2 & 27 & 0.00002 & N. E. E. \\
\hline 6 al 12 detto..... & 763.1 & 760.1 & 23 & 28.1 & 26.2 & 25.4 & 0.00015 & E. N. E. \\
\hline 13 al 19 detto. . . . & 762.1 & 760 & 24 & 28.1 & 27.2 & 27 & - & N. E. E. \\
\hline 20 al 26 detto. . . . & 763.1 & 760.1 & 24 & 28.2 & 252 & 25 & - & E. N. E. \\
\hline 27 detto al 2 agosto. & 762.5 & 760 & 18 & 29 & 25 & 25 & - & O. N.E. \\
\hline 3 al 9 detto $\ldots$. & 762.1 & 760.1 & 24.1 & 29 & 26.2 & 25.1 & - & E. N.E. \\
\hline 10 al 16 detto. . . . & 7631 & 760.1 & 22 & 29 & 25.2 & 25 & - & E. N.E. \\
\hline 17 al 23 detto. . & 763.1 & 760.1 & 23 & 28.2 & 25.2 & 25 & - & E. O. N.E. \\
\hline 24 al 30 detto. . & 762.1 & 760.1 & 24 & 29 & 25.2 & 25 & - & E. O. N. F. \\
\hline 31 detto al 6 settemb. & 7630 & 761 & 21 & 29 & 25.2 & 25.1 & 0.00024 & E. N.E. \\
\hline 7 al 13 detto. .... & 763.2 & 760.5 & 24.1 & 28.2 & 27.2 & 26.4 & - & N. E. O. N. O. E. \\
\hline 14 al 20 detto & 7631 & 760.1 & 24 & 30 & 27.2 & 27 & - & O. N.E. \\
\hline 21 al $2 \%$ detto. . & 763.1 & 760.1 & 24 & 30 & 27.4 & 27.1 & - & E. N. E. O. \\
\hline 28 detto al 4 ottobre. & 763.2 & 760.1 & 24.2 & 29.2 & 27.4 & 27 & - & E. N.E. \\
\hline 5 all'll detto .... & 763.1 & 761.2 & 24 & 29.2 & 27.4 & 27.2 & -- & E. N. E. \\
\hline 12 al 18 detto... & 761.2 & 762.1 & 25 & 30 & 27.4 & 27 & - & N. E. \\
\hline 19 al 25 detto. . . . & 765.1 & 764.1 & 24 & 30 & 27.4 & 27.1 & $0.0006 j$ & E. N.E. \\
\hline 26 detto al $1^{\circ}$ novemb. & 764.5 & 760.1 & 24 & 29 & 27.2 & 27 & - & E. N. E. \\
\hline 2 all's detto . . . . & 7641 & 760.1 & 24 & 30 & 27.4 & 27.1 & $0.000: 9$ & E. N. E. \\
\hline 9 al 15 detto. & 764.1 & 763.2 & 24 & 30 & 27.4 & 27 & - & E. N.E. \\
\hline 16 al 22 detto. . & $764 . \mathrm{i}$ & 762.4 & 24 & 30 & 27.4 & 27 & 0.00027 & E. N. E. \\
\hline 23 al 29 detto & 764.2 & 762.1 & 24 & 30 & 27.4 & 27.2 & 0.00297 & E. N.E. \\
\hline 30 detto al 6 dicemb. & 765.1 & 760.1 & 24.1 & 30 & 27.2 & 27.1 & 0.00080 & E. N. E. \\
\hline 7 al 13 detto. . . . & 764.1 & 762.1 & 24 & 30 & 27.2 & 25.6 & 0.00164 & E. N. E. \\
\hline 14 al 20 detto & 7641 & 762 & 241 & 30 & 27.2 & 26.7 & 0.00206 & O. N. O. \\
\hline 21 al 27 detto. & 765.1 & 761.2 & 24.4 & 30.4 & 27.4 & 26.4 & 0.00135 & E. N.O. \\
\hline
\end{tabular}


FIGI 



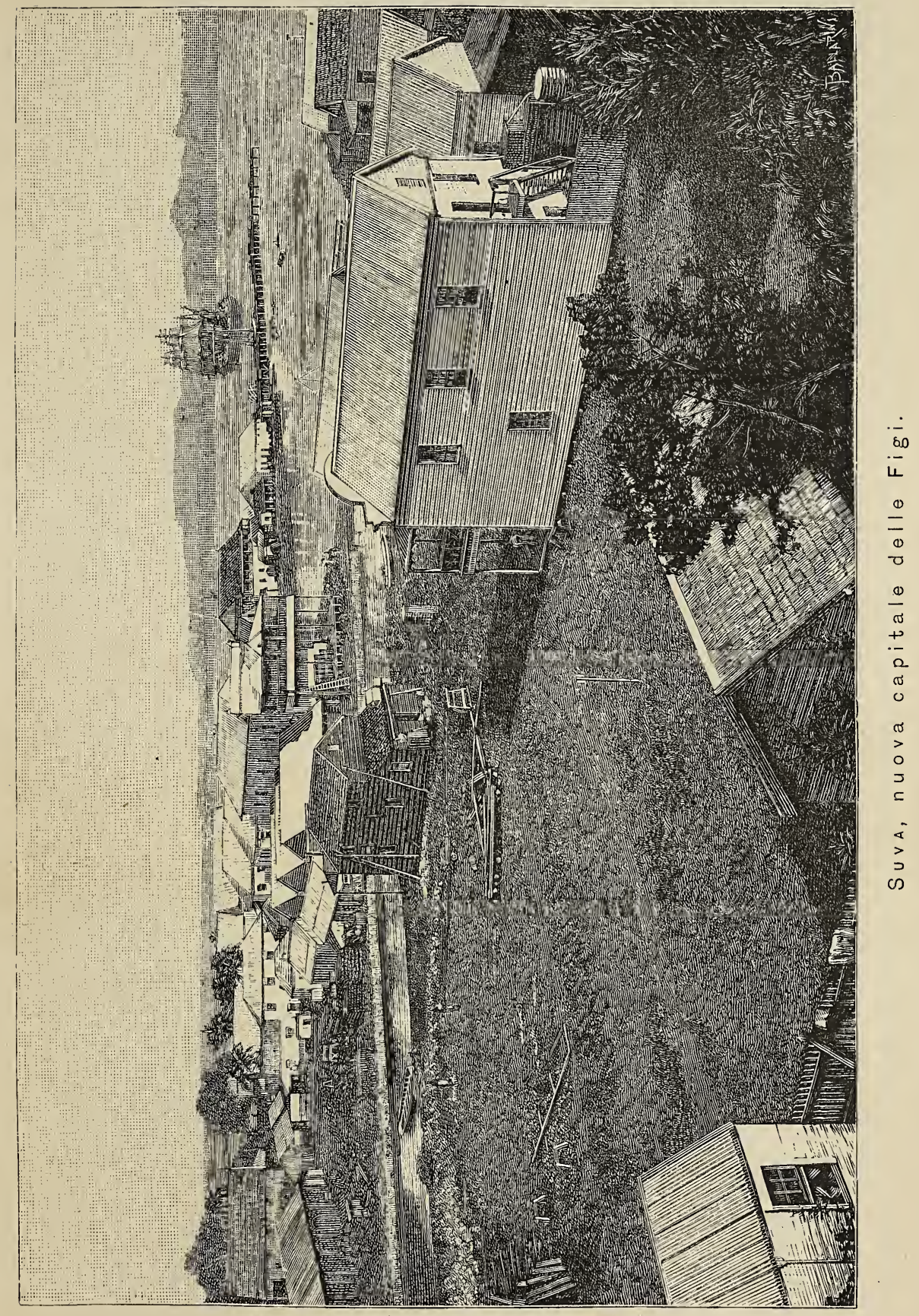





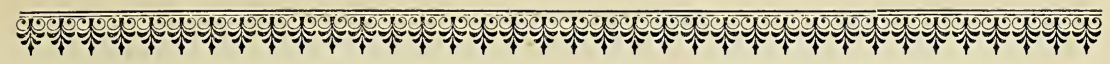

\section{CAPITOLO I.}

R E M I I I S C E N Z F

I.

\section{Approdo alle Figi - Il Capitano Ferrea.}

Le istruzioni del Ministero della Marina lasciavano alla Caracciolo la scelta di approdare, proseguendo per Sydney, in uno degli arcipelaghi di Samoa, di Tonga o delle Figi. La scelta cadde su quest'ultimo che, per l'importanza acquistata con l'occupazione inglese, sembrava il più degno di essere visitato. A questo motivo aggiungevasi inoltre il desiderio di attingere, sui luoghi, precise informazioni intorno a' particolari dell' infelice sorte toccata, nei paraggi figiani, sul principio del 1853, ad un nostro concittadino, il Capitano Giacomo Ferrea.

Il Ferrea, era nato a Nervi di Liguria. Di verde età, ardito quanto intraprendente marino, comandava il brigantino Rosa, di cui era pure armatore. Avea salpato dal Callao nell'aprile del 1852, in zavorra di sale, veleggiando alla volta di Australia, alla ventura, ossia in cerca di noli. Giunto a Sydney nel periodo febbrile della scoperta dell'oro, non fu che dopo aver superato infinite difficoltà ch'egli potè noleggiarsi, nel novembre đello stesso anno, per 
una delle isole Figi, con un carico di materiali destinati alla costruzione di una chiesa della Missione cattolica francese. Il Ferrea portava seco una vistosa paccotiglia di ferramenta, vecchie armi, tabacco, ecc.... che si riprometteva barattare con i prodotti indigeni. Al suo arrivo nelle Figi, messo a terra il suo carico, egli riesciva tosto ad incettare una buona quantità di olio di cocco. Sventuratamente, la vivacità del suo carattere insieme con la perfidia degli indigeni, suscitarono, sulla coperta della sua nave, animatissimo diverbio, che trascese in una lotta a corpo a corpo fra l'equipaggio ed i figiani, e finalmente in un'orrenda carneficina. Il povero Ferrea e tutto il suo equipaggio, tranne il nipote del capitano che fu coperto di ferite, e tre marinai rimasti nella stiva, armati di fucile, furono massacrati. Gl' indigeni, perduti molti de' loro, abbandonarono, gettandosi al nuoto, il brigantino Rosa, che i superstiti si affrettarono di mettere alla vela.

Dopo una traversata di quaranta giorni, in balia del vento, quella nave era sorpresa, nelle vicinanze di Sydney, da un forte temporale e naufragava sulla costa; peró i suoi ardimentosi nocchieri riuscirono a salvarsi.

Del Capitano Ferrea, della sua nave, del terribile dramma di Figi poco o nulla si parlò. Egli, che primo avea fatto sventolare i colori nazionali in Australia e nelle Figi, in tempi in cui la navigazione oceanica presentava innumerevoli pericoli, specie per navi a vela, meritava di essere da noi ricordato, e la premura nostra d'indagare le vicende degli ultimi istanti della sua esistenza, per far onorare la memoria di lui, era pienamente giustificata. 
II.

Sura - Lami.

Scelte le Figi quale approdo successivo a quello di Tahiti, si decise di prendere porto a Suva, recentemente divenuta capitale dell'arcipelago invece di Levuka, esautorata per influsso di cause complesse, fra le quali, ostacoli topografici ad uno sviluppo conveniente, esigenze pecuniarie eccessive de'missionari wesleyani, per cedere al Governo coloniale i terreni richiesti con fini d'interesse pubblico.

Gli oppositori al trasferimento della capitale facevano osservare che il clima di Levuka è delizioso per ventilazione e per serenità di cielo, e che, posta al centro d'immense piantagioni di cocco, Levuka, rappresenta il naturale emporio del commercio dell'olio e del copra, mentre Suva va soggetta a pioggie incessanti e non ha piante di cocco.

Noi non anticiperemo giudizi, e tacendo delle risorse zuccherine di Suva e dintorni, accenneremo soltanto che questa localitá puó sembrare, meglio di Levuka, collocata sul corso delle grandi arterie del movimento marittimo mondiale.

All'alba del 18 agosto eravamo in vista dell'ancoraggio di Rewa, le cui apparenze ci fecero, a prima vista, credere di trovarci a Suva. L'inganno fu presto chiarito, e, percorso un certo tratto, parallelamente alla barriera corallina, che cinge l'isola, si entrò nel magnifico porto naturale della vera Suva.

La nuova capitale delle Figi è ancora in embrione, appena abbozzata. I caseggiati, già numerosi ed allineati secondo un piano regolatore, attestano la fretta con la quale furono costruiti: sono di legname, e, comunemente, smontabili. Lungo la riva i magazzini, le botteghe, gli uffici, le banche; a monte, sopra verdeggiante 
collina, le chiese, l'ospedale, le carceri, le abitazioni de' coloni, la pittoresca casa del Governatore, che isolata domina il mare. Non rassomiglia a Papeete, la capitale di Tahiti, ove la convivenza promiscua del bianco con le razze di colore determina una fisonomia locale bizzarra e gaia. A Suva, come in ogni colonia britannica, il bianco vive completamente separato dall'indigeno, ha la sua propria città, e la capitale figiana porta l'impronta inglese: è compassata, seria, forse monotona.

Il contrasto tra le due capitali dell'Oceania dá la nota del temperamento diverso de' due popoli colonizzatori: l'uno, il francese, conquista e poi si getta spensierato, senza ritegno, in mezzo alla gente soggiogata, solleticandone la vanità, trastullandosi delle sue debolezze, e stringendole i polsi con il regime militare ed il protezionismo economico; l'altro, l'inglese, s'insedia da padrone laddove il suo interesse ed il sentimento della sua superioritá lo chiamano, evitando gelosamente il contatto immediato degli abitanti del suolo, ma educandoli, coltivandone le buone attitudini e tentando fare di essi de' produttori e de' consumatori valevoli per i suoi mercati, con il puntello di una larga libertá economica.

La colonia inglese di Suva si compone, in gran parte, di bianchi australiani del rito ebraico.

Il Governatore sir Des Vœux non poteva darci le feste tahitiane di luglio; nonostante, fece del suo meglio per renderci gradito il soggiorno delle Figi, e nello insistere perchè si accettasse l'ospitalità in casa sua, diede chiara prova della sua simpatia per la Caracciolo. Una sera egli e l'egregia famiglia sua ci fecero assistere ad un ballo figiano, attori i soldati della sua guardia di onore, che è altresì la guarnigione di Suva ed il nerbo principale dell'armata figiana: 'sessanta bellissimi indigeni sui centoventi che l'armata medesima conta. Il ballo era la rappresentazione mimicocoreografica di scene della natura: le onde che irrompono sui çoralli; gli uccelli che amoreggiano. L'esecuzione fu splendida per la varietá delle figure, la precisione delle mosse, il ritmo e 
la cadenza del canto, e venne quindi ripetutamente applaudita dalle nostre grida di venaka! venaka! - buono! buono! Chiuse il ballo la preparazione di una tinozza di kava, che i danzatori guerrieri consumarono con infinito contento. Le donne degli ufficiali indigeni, per singolare cortesia, si erano degnate di masticare loro stesse le radici, da cui escì la narcotica bevanda.

La domenica seguente rivedemmo, ne' loro abiti di festa, que' baldi soldati sotto le armi, passati in rivista dal proprio Comandante, che è M.r Marriotte, garbato giovinotto inglese. Sono veramente magnifici, cinti di sciabola e giberna, con il parau rosso, la giacca turchina scollata, a maniche corte e fregiata di rosso. Nei giorni feriali essi hanno il busto nudo, con il solo parau rosso. La carabina Schneider, di cui vanno superbi - bisogna confessarlo la maneggiano con la disinvoltura e la maestria di veterani, e le evoluzioni militari le compiono con l'agilità, la grazia e l'ordine che li distinguono nelle danze. Finita la rivista, li seguimmo al loro tempio protestante, ove li aspettava un vecchio pastore indigeno per ispiegare con enfasi versetti di circostanza della Bibbia. Sdraiati sopra stuoie, lo ascoltavano silenziosi, assai pii penetrati, apparentemente almeno, della sua fluida ed animata eloquenza, che delle profonde verità bibliche.

Le forze militari figiane che, come si disse, sono rappresentate da 120 uomini, formano due compagnie, comandata ciascuna da un ufficiale inglese; le mezze compagnie sono comandate da ufficiali indigeni. Il comando generale ne è affidato all'Aiutante di campo del Governatore, che è pure inglese. Al Governatore è devoluto il diritto di nomina di tali ufficiali. Attualmente la seconda compagnia trovasi nell'interno dell'isola Viti-Levu, per sorvegliare gl'irrequieti montanari.

Il villaggio indigeno più vicino a Suva chiamasi Lami: è in fondo della baia, ma non visibile dal mare, stante la folta vegetazione ed il colore oscuro delle capanne.

Gli ufficiali della Caracciolo, che spinti dalla curiosità vi si recarono, al loro arrivo nel villaggio, s'imbatterono in un gruppo 
di ninfe dai contorni statuari, bagnantisi in costume preadamitico nelle acque stagnanti di un gran fosso. I sorrisi di esse, dovuti alla sorpresa ed anche alla civetteria selvaggia, furono interrotti dall'apparizione improvvisa di un bel figiano, alto e tarchiato, che propose di servir di guida agli ufficiali. Questi abbandonarono la scena piccante e andarono a vedere il principale edificio di Lami: la chiesa-scuola, il fabbricato che alternativamente serve al doppio ufficio di chiesa e di scuola. La costruzione ne è semplice e solida: forma rettangolare, pareti basse con ossatura di legname intrecciato di bambú e rivestito di foglie; tetto mono-cuspidale, molto inclinato ed impermeabile mercè foglie di pandanus, finamente ripiegate in quattro. Ad ogni lato dell'edifizio un'apertura molto bassa, onde conviene chinarsi per entrare. Le altre case di Lami sono foggiate sullo stile della chiesa-scuola, ma non hanno che una sola porta. I nostri ufficiali penetrarono in alcune e vi furono ricevuti con segni manifesti di compiacenza. Pochi mobili in quelle case: grandi stuoie, ben lavorate, stese al suolo sopra spesso strato di foglie secche; in un angolo il focolare; appesi alle pareti ed al letto, gli utensili, le stuoie nuove, le armi, molte lance da pesca. La popolazione vestiva con molta semplicita; gli uomini erano in maglia con il parau, le donne con il parau ed una camiciuola scollata e con maniche corte. Tutti a capo scoperto, ma con i capelli lunghi e ritti stranamente acconciati. Narrasi che i missionari, per amore di nettezza, consigliassero un tempo a' loro neofiti figiani di portare i capelli corti, ed il consiglio ebbe gravi conseguenze; non piu protetti contro l'ardore de'raggi solari, i figiani si-ammalarono. 
II.

\section{Escursione a Nausori ed a Rewa - L'agente consolare italiano Partenza della "Caracciolo,}

Sulla sponda sinistra del fiume di Rewa, nel sito detto di Nausori, a sedici miglia da Suva, il solerte ingegnere scozzese $\mathrm{M}^{\mathrm{r}}$ Fairgreive impiantava, l'anno scorso, in nome di una Societá di capitalisti australiani, uno stabilimento per la fabbricazione dello zucchero greggio, stabilimento che, funzionando da soli sette mesi, oggi può considerarsi come il più vasto de' suoi congeneri nel Pacifico e forse in ogni altra parte del globo.

Il prendere notizia da vicino della grandiosa impresa, solcando il Rewa, uno de' fiumi maggiori dell'arcipelago, e visitare la città indigena omonima, teatro in addietro di tanti eccidî, erano allettamenti sufficienti per suggerire una gita a Nausori. Infatti, la mattina del 24, la barca a vapore della Caracciolo con gli ufficiali dilettanti di escursioni, dirigeva, senza uscire dalla baia di Suva, verso una delle quattro bocche del delta di Rewa.

Rive basse, ma ricchissime di vegetazione. Il fiume piega rapidamente all'Est e mostra una pittoresca isoletta, che deve costeggiarsi a Sud per poi volgere a N. E. e continuare a risalire il fiume. Scoperta del tutto l'isoletta, presentasi a destra la Missione cattolica con la chiesa e i caseggiati attigui. Da questo punto il fiume corre a N. O. fino a Nausori.

É poco più in su della Missione che s'incominciano a vedere estesi campi di canne di zucchero, le quali, perchè coltivate e nutrite da profondi terreni alluvionali, rimpiccioliscono, nel confronto, le esili canne di zucchero selvaggie, crescenti sui margini della costa. Giova il dire che codesta conquista dell'arte sulla natura appartiene esclusivamente a' figiani, ottimi agricoltori. 
Sul passaggio della barca a vapore, che aveva la sua bandiera spiegata, or qua or là, accorrevano in frotte alla riva gli indigeni salutando con grida di gioia. Alcune piroghe si spingevano a monte puntando i remi sul fondo. In una di queste emigrava un'intera famiglia: sulla piattaforma erano le suppellettili di casa; un vecchio dirigeva la manovra; maneggiava con vigoria e destrezza il remo una bella giovanetta, e nel fondo della piroga un adulto aggottava.

$\mathrm{Fu}$ dopo quattro ore di viaggio che i nostri ufficiali arrivarono a Nausori, quivi cordialmente ricevuti dall'ingegnere Fairgreive, per il quale avevano commendatizie del gentile signor Amherst, segretario particolare di S. E. il Governatore.

Prima di esaminare lo stabilimento fecero una piccola sosta all'albergo, che è sulla sponda opposta e precisamente al confluente del Rewa con il suo tributario, il Waimann, navigabile per un traito di 10 miglia. Dall'alto dell'albergo si ammira la ridente campagna bagnata dai due fiumi, e lo sguardo si ferma con particolare predilezione sulla pianura ondulata, che, coperta quasi tutta da canne di zucchero, sposa armoniosamente le sue tinte verdi chiare con quelle piu cupe delle lontane colline.

Il signor Fairgreive volle in persona fare gli onori di casa, assistito da uno de' suoi dipendenti, un giovane belga. L'esame dello stabilimento fu lungo, minuzioso ed oltremodo interessante, destando' meraviglia l'assetto dato e la produzione ottenuta in così poco tempo. Gl' indigeni, amanti di lavorare sull' acqua, erano intenti a togliere dai pontoni galleggianti la canna di zucchero che una tela metallica continua portava agli stritolatoi; li dirigeva uno de' loro Capi, dacchè i figiani sono restii ad ubbidire agli ordini immediati de' bianchi. Erano montanari di colore piủ scuro di quello dei figiani di Suva, con il parau taluni, altri ridotti a rimpiazzarlo con semplici foglie; avevano il viso tinto di nero o di bianco, per abbellimento; i denti bianchi come l'avorio.

Il villaggio di Nausori non presenta alcune particolarità di 


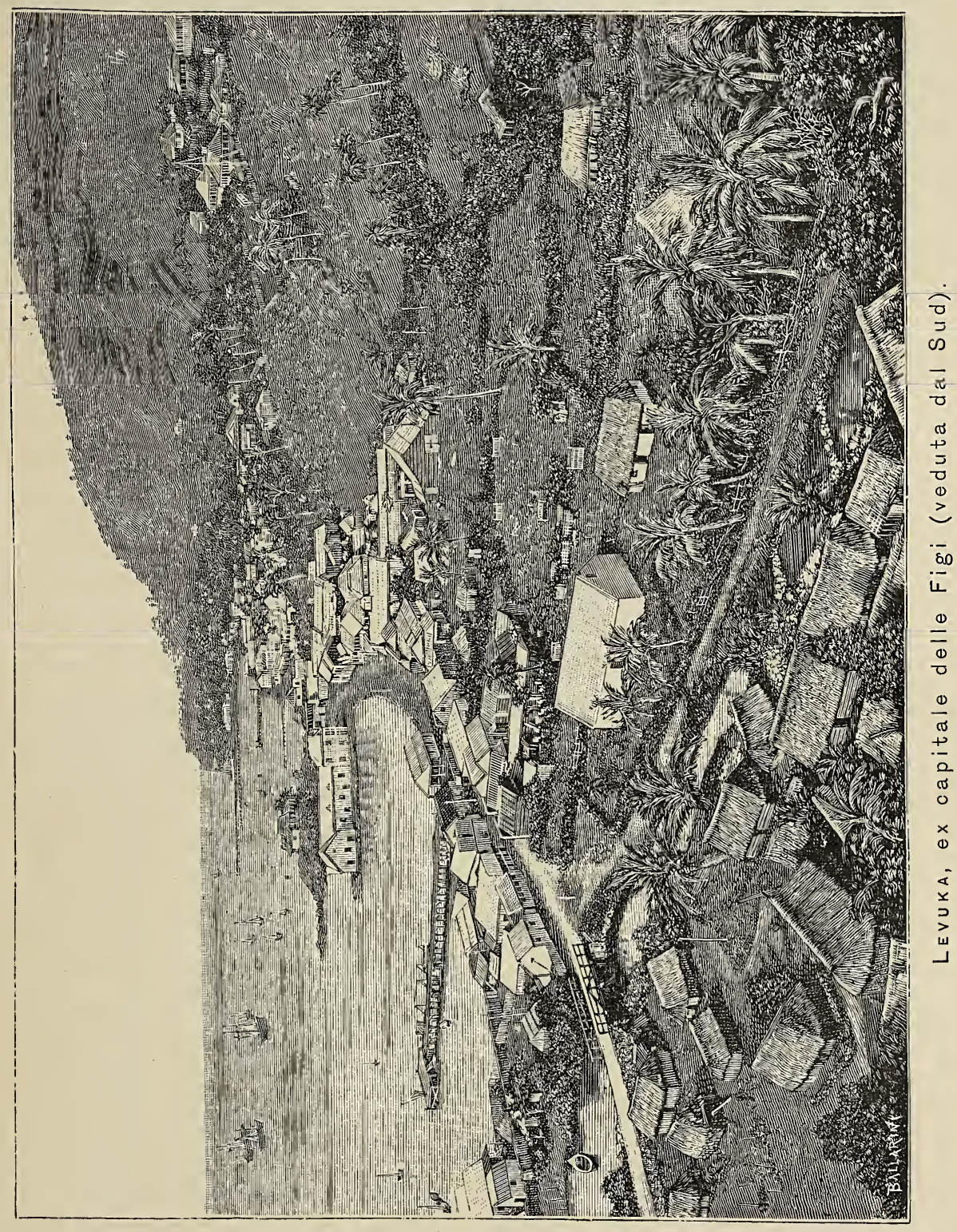



rilıevo; è un aggregato di capanne intorno ad una più grande e di una certa eleganza: quella appartenente al Capo.

Il domani i nostri ufficiali ridiscesero il fiume con la barca a vapore per visitare Rewa, accompagnati dal signor Fairgreive e da uno de' figli del Capo di quella città.

Rewa giace sopra un isolotto, nel punto in cui il fiume si biforca, per formare la terza bocca del delta Wai-ni-ki. Un canale di due miglia di lunghezza e largo 20 metri, opera indigena, mette Rewa in comunicazione con Mbau, altra città storica figiana.

Sul principio del secolo, Rewa s'innalzo a grande fortuna fra delitti di sangue atroci, per gelosia di donne ed ambizione dei Capi. Fu la rivale di Mbau, finchè Thakombau, Signore di questa, non l'ebbe posta in servitu, vendicandosi del Capo Vasu-Rawalisu, che aveva ordito contro di lui micidiale congiura.

Un nugolo di fanciulli diede il benvenuto, sulla spiaggia di Rewa, ai passeggeri della nostra barca, che, scesi a terra, andarono immantinenti in cerca del Capo del paese. Lo trovarono nella. sua abitazione lieto di stringere loro la mano. Egli è un ercole figiano, uso al comando; ha la fronte ampia dinotante non comune intelligenza; la moglie, terza compagna sua, di belle fattezze, giovane e simpatica, sedeva poco discosta da lui; due indigeni stendevano sulla carta gli ordini ch'egli dettava. La casa era spaziosa e comoda.

Il Capo di Rewa condusse i suoi ospiti a fare un giro nel paese, precedendoli, maestoso nel portamento, ed annunziando di tratto in tratto ad alta voce la loro qualità: veduto ed ascoltato dall' interno delle capanne, nessuno si affacciava a guardare; e se qualcuno passava cedeva immediatamente il passo, deponendo il suo fardello, in segno di rispetto.

Meno riverenti furono alcune megere, che circondarono improvvisamente la comitiva europea per offrirle, con istrepito, l'acquisto di graziosi esemplari della ceramica indigena.

Il paese era bello con le sue capanne erette in mezzo ad un suolo ferace e molto ben coltivato. Dopo averlo percorso in tutti 
i sensi, il Capo di Rewa ed i suoi ospiti si recarono alla Missione. cattolica di Rewa, ove trovarono vecchi sacerdoti francesi che da. 40 anni esercitano, nell'arcipelago figiano, il loro nobile ministero, senza rammarichi e rassegnati a non riveder più mai la loro terra nativa. Quanta fede, quanta rassegnazione in quei venerandi missionari!

L'ora della partenza essendo giunta, i nostri ufficiali dovettero accomiatarsi dai missionari, dal Capo di Rewa e dal signor Fairgreive, che ritornava al suo stabilimento di Nausori, ed imbarcarsi per rientrare prima di notte a Suva.

Ricevemmo il giovedi, 25 agosto, una visita inaspettata e cara. Il nostro Agente consolare nelle Figi, l'ingegnere Martelli, dall' isola Koro, nella quale egli possiede terreni ed abita da 10 anni, venne a Suva, appositamente per vederci. Bell'uomo sulla sessantina, ex militare, ex deputato subalpino, ex intendente di Cagliari, fregiato della medaglia d'oro al valore civile, salì sulla Caracciolo visibilmente commosso. Dal 1869 egli non aveva piu veduto la bandiera del suo paese sventolare sopra una nave da guerra!

Il Martelli ama codeste isole, elogia parcamente i figiani e vanta le ricchezze agricole dell'arcipelago. È convinto che alcune famiglie di agricoltori italiani troverebbero il loro tornaconto a dedicare le fatiche del braccio nell' arcipelago di Figi. Avrà ragione, ma come consigliare della brava gente a trasportare, con sacrifizi gravi di danaro, i suoi penati in una località lontanissima dalla patria, per un avvenire molto ignoto, nello svolgimento delle sue fasi? Quanti altri siti più vicini o più ricchi non si presentano mai all'attività dei nostri laboriosi emigranti?

Il signor Martelli ci fece dono di vari oggetti indigeni, che unimmo alle nostre collezioni di viaggio.

Con dispiacere dobbiamo dire che riuscirono infruttuose le nostre indagini riguardo all'evento Ferrea; il Governo, le Missioni religiose, gli anziani residenti europei, interpellati in proposito, non ebbero che esclamazioni di sorpresa! Il prelodato signor 


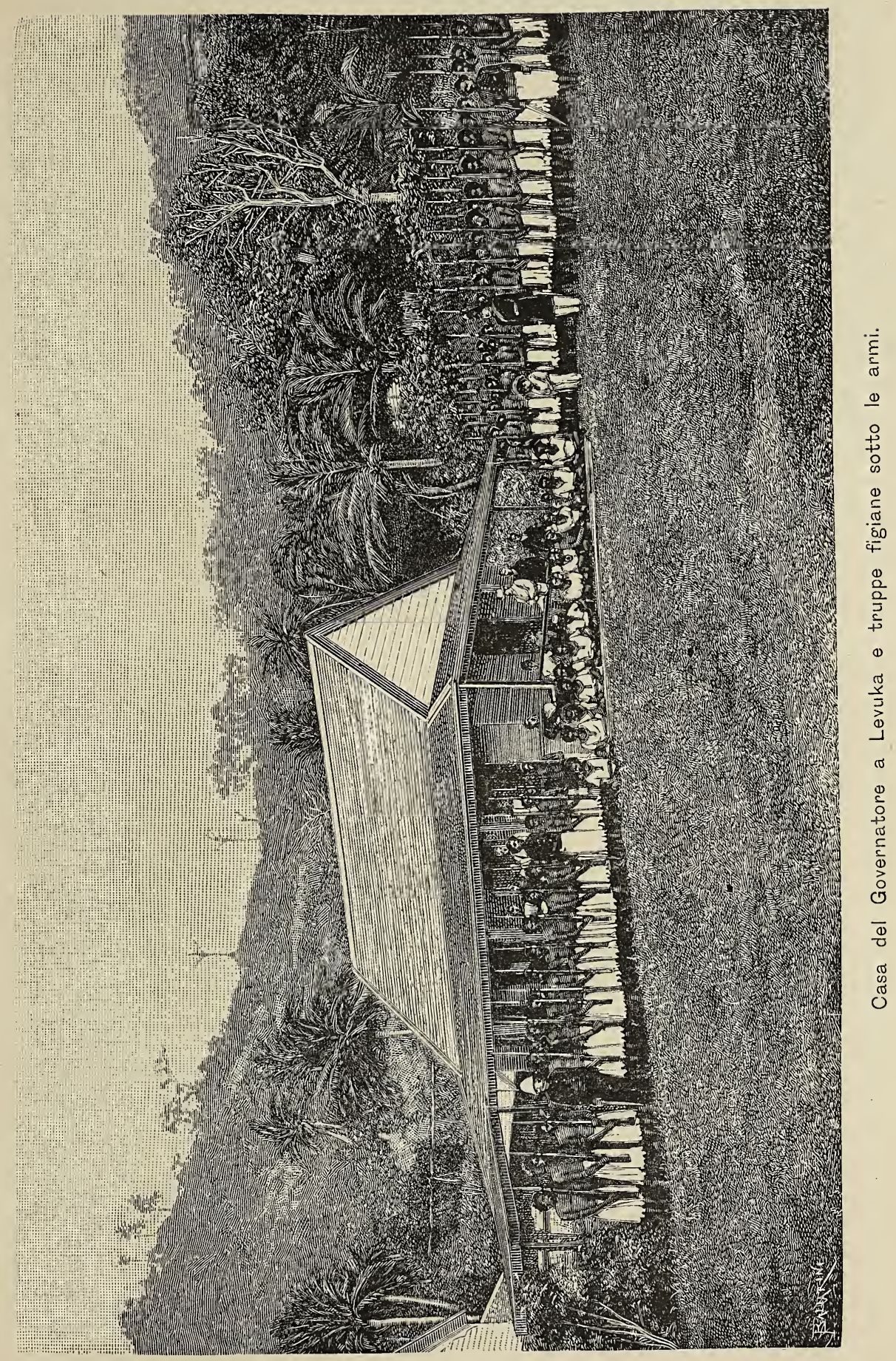



Martelli prenderà informazioni e ci terrà al corrente del risultato delle sue ricerche. ${ }^{1}$

Il dì 27 agosto pioveva a catinelle, ed aveva piovuto in tal guisa sino dal giorno del nostro arrivo! Non potevamo aspettare che il bel tempo ci facesse godere il panorama di Suva, illuminato dal sole; Sydney ci aspettava, e noi partimmo salutando con l'inno inglese la bandiera del Governatore.

III.

\section{Stabilimento di Nausori della "Colonial Sugar Refining Company" di New South Wales e Figi.}

Il superbo stabilimento di Nausori, situato a 50 metri dal fiume, consta, principalmente, di un vasto recinto chiuso e coperto di tettoie, di varie motrici e di numerose macchine utensili, come stritolatoi, purificatoi, filtri, evaporizzatori, ecc.

Delle macchine motrici quattro di 40 cavalli di forza ciascuna animano gli stritolatoi, e tre di minor potenza fanno agire le grandi trombe pneumatiche e di prosciugamento.

Quattordici barche della portata di 70 tonnellate ognuna, e sette rimorchiatori, uno dei quali ad elica, sono adibiti al trasporto delle canne e dello zucchero, per la via di mare, mentre i treni percorrenti un tronco di ferrovia dell'estensione di 15 miglia con varie diramazioni di binarî, provvedono al trasporto delle canne dalle diverse piantagioni, per la via di terra.

Quando tutti gli apparati funzionano, la produzione della fab-

1 Nelle sue più recenti lettere il Martelli ci scriveva che le sue ricerche non hanno avuto alcun risultato. 
brica ascende, nelle 24 ore, a 60 tonnellate di zucchero, corrispondenti a 900 tonnellate di canne, ma la media giornaliera non supera le 45 tonnellate di zucchero.

$L \in$ macchine sono in azione giorno e notte, tranne la domenica.

Il taglio delle canne, effettuandosi semestralmente, le macchine non agiscono che durante sei mesi; nel rimanente dell'anno si dà corso alle necessarie riparazioni e si prepara l'occorrente per ricevere il nuovo raccolto.

I terreni dello stabilimento sarebbero insufficienti ad alimentare la fabbrica; quindi la Società fa contratti con i Capi delle tribú indigene per ottenere vistose quantità di canne.

Lo stabilimento annovera un migliaio di lavoranti tra figiani, indiani ed europei. Questi ultimi, impiegati esclusivamente alle scritturazioni ed al servizio delle macchine, sono in piccolo numero, 200 circa.

Bianchi ed uomini di colore ricevono dalla Società alloggio e vitto, essendo, in caso di bisogno, ricoverati in un ospedale della Società stessa.

Ecco ora il processo di fabbricazione dello zucchero seguito dallo stabilimento: ${ }^{1}$

Cariche di canne, le barche vengono collocate ai lati di due larghe cinghie continue protendentisi dall'interno delle officine circa 20 metri sul fiume. Le canne sono allora gettate sulle cinghie, e trascinate dal moto continuo ne' rispettivi stritolatoi, da cui cade il sugo in vasche praticate nel suolo.

La parte lignea uscita dal primo stritolatoio, previo annaffiamento con acqua calda, passa in un secondo, e si arresta sopra una piattaforma, che, forata, la distribuisce come combustibile ad otto generatori.

Il sugo è fatto salire in un gran serbatoio, e successivamente in altri, onde subire, per sedimento, una prima e naturale separazione.

1 Vedi tavola. 


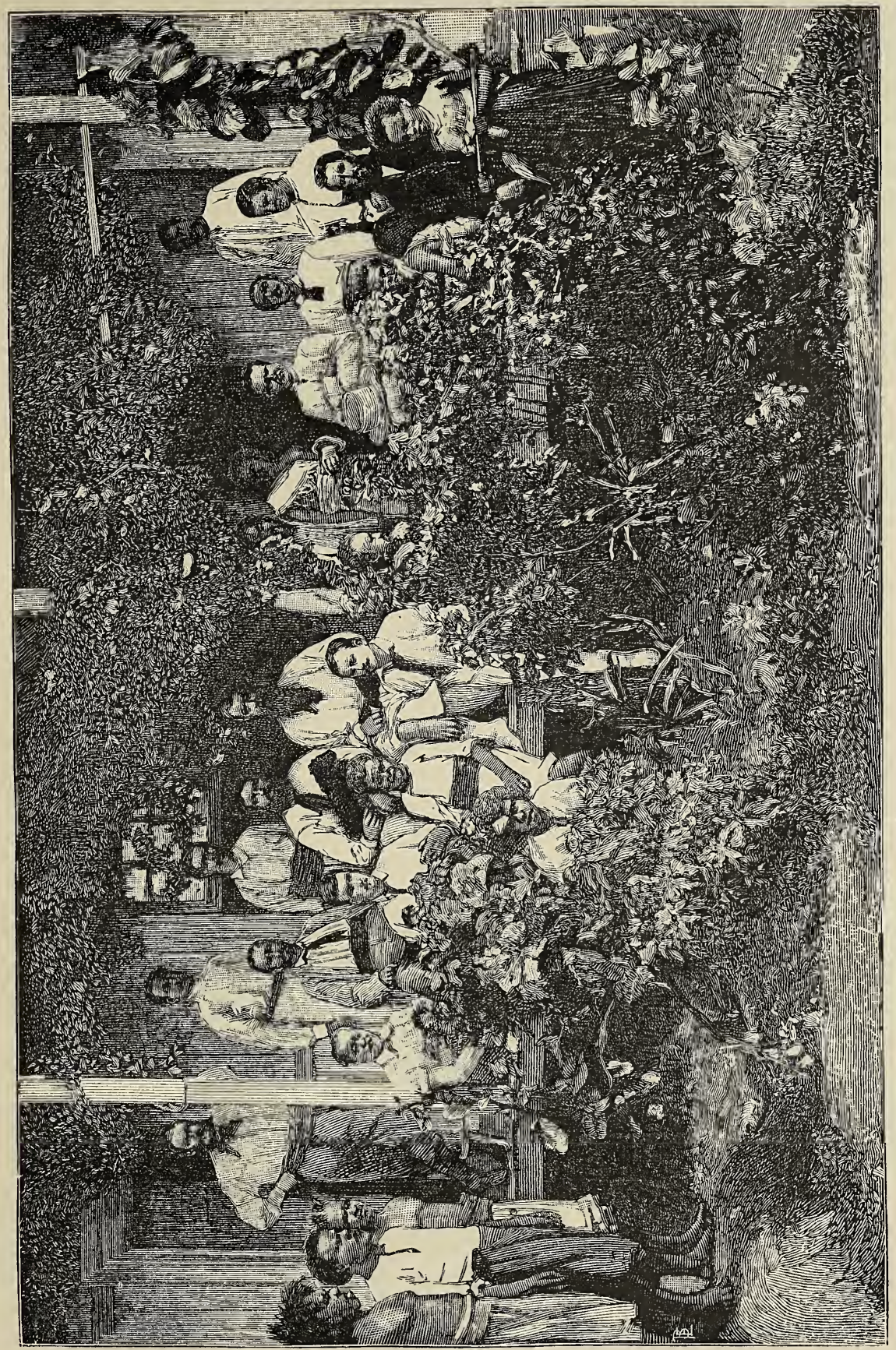

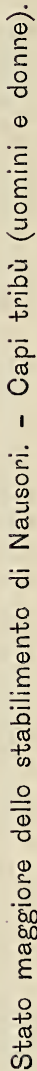



Da questi serbatoi lo s'immette, aggiuntovi una lieve dose di calce, ${ }^{1}$ in una caldaia, per essere riscaldato e travasato sotto la pressione del vapore in recipienti circolari, a doppio fondo, entro cui una corrente continua di vapore stabilisce una costante ed elevata temperatura. Intanto le materie albuminose si coagulano, arricchendosi di altre materie eterogenee, e vengono a galla sotto forma di schiuma, che si toglie, a mano a mano, con grossi cucchiaioni.

Trascorso un certo tempo, quando il liquido non presenta pii impurità alla superficie, lo si travasa facendolo passare, da un foro praticato nel doppio fondo, in nuovi recipienti sottostanti, per farlo depositare. In questi ultimi, un tubo, che è articolato ad una estremitá sul fondo, e porta all'altra un galleggiante, mette al momento voluto in comunicazione gli strati superiori del liquido con i feltri. Quando il liquido è abbastanza chiarificato, si abbassa quel tubo lasciando scendere in esso la parte priva di depositi e facendole traversare un filtro semplice, formato, cioè, di un panno di lana e di uno strato di carbone animale, per poterlo sottoporre alla evaporizzazione. La parte densa del liquido corre invece in ispeciali filtri, in cui depone ogni materia calcarea.

Il liquido zuccherino durante il suo passaggio ne' differenti serbatoi è mantenuto ad una temperatura di $82^{\circ} 5 \mathrm{C}^{\circ}$ circa.

Tutto il sugo, in guise diverse filtrato, è riunito in un medesimo recipiente, e poi aspirato dal primo degli evaporizzatori. ${ }^{2}$ Questi sono in numero di sette, ma per ogni operazione, salvo casi di straordinaria abbondanza di liquido, se ne adoperano solamente quattro.

${ }^{1}$ La calce si combina con l'acido carbonico che contiene il liquido e dà origine ad un carbonato di calce, il quale precipitandosi trascina seco altre impurità, e specialmente le sostanze coloranti.

2 Sono recipienti cilindrici, ne' quali una corrente di vapore traversa lo esterno di un fascio di tubi entro cui si trova il sugo. 
Gli evaporizzatori si trovano in comunicazione tra loro ed altresì con le trombe pneumatiche, ${ }^{1}$ con il generatore del vapore circolante e con il tubo d'invio della sostanza zuccherina. Per tal modo, mentre vapore e sugo entrano nel primo evaporizzatore, l'aspirazione delle trombe si fa nel quarto, ${ }^{2}$ e le due correnti gassose seguono una medesima direzione con decrescente intensità. La temperatura circolante scende gradatamente nel quarto evaporizzatore da $82^{\circ} 4 \mathrm{C}^{\circ}$ a $65^{\circ} 7 \mathrm{C}^{\circ}$, ed il vuoto da 29 centimetri scende anche a poco a poco a 5 centimetri nel primo.

Tosto che il sugo ha acquistato una densità di $35^{\circ}$ dell'areometro Baumé, lo si fa scendere allo stato di miele dal quarto evaporizzatore in un cilindro chiuso. Da questo, per mezzo di pressione di vapore, il miele è distribuito in una serie di casse collocate in alto, dalle quali è aspirato dal vuoto esistente in un unico e grande serbatoio, detto caldaia a vuoto, che ha forma di pera ed un doppio fondo per la circolazione del vapore. Il prodotto così ottenuto è portato agli asciugatoi a forza centrifuga, che separano lo sciroppo dalla parte cristallina, ossia dallo zucchero. Lo sciroppo cola in un pozzo e ritorna nella caldaia a vuoto per un'ultima estrazione zuccherina, e passa finalmente in casse, che si tengono esposte all'aria, fino a che la parte acquosa si sia evaporizzata e non rimanga che un denso strato di melassa.

La melassa, chiusa in botti, è spedita negli Stati Uniti, ove si fa con essa del rhum ed altri liquori. Lo zucchero è insaccato e mandata in Australia alle raffinerie della Società.

Può dirsi conchiudendo che lo stabilimento di Nausori è uno de' piu importanti, se non il piu importante di tutti i suoi con-

${ }^{1}$ Le trombe pneumatiche aspirano il vapore generato dalla parte acquosa del sugo.

${ }^{2}$ Ad un certo punto del tubo di comunicazione delle trombe pneumatiche vi ha un separatore, che impedisce al sugo di arrivare sino alle trombe, se per avventura si verificassero ebollizioni tumultuose negli apparati evaporatori. 
generi, sia per l'abbondanza della produzione come per il perfezionamento del macchinario. È superiore a quello di S. Clara sulla linea Lima-Orosa (Perü), giustamente reputato come uno de' piu produttivi che si conoscano. Nausori, a differenza di S. Clara, possiede un secondo stritolatoio per viemmeglio estrarre dalle canne la parte zuccherina, impiega filtri a pressione per ottenere maggiore quantità di zucchero da' residui della parte sugosa, e adopera un' unica caldaia a vuoto, anzichè varie con tubi a serpentina contenenti il vapore. 



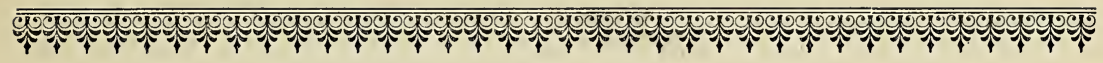

\title{
CAPITOLO II.
}

\author{
C E N I I STORICI
}

I.

Confini dell'arcipelago di Figi - Sua scoperta - I primi bianchi.

L'arcipelago delle Figi abbraccia tutte le terre, isole, scogli, banchi, compresi tra il $15^{\circ}$ ed il $20^{\circ}$ grado di latitudine Sud, e fra il $177^{\circ}$ grado di longitudine Ovest ed il $175^{\circ}$ di longitudine Est di Greenwich, occupando quindi un'area di circa 198,025 miglia geografiche quadrate. Dista da Sydney 1,900 miglia, 300 dall'arcipelago di Tonga e 500 dall'arcipelago di Samoa. Annovera pressochè 259 isole, 80 delle quali abitate; le maggiori essendo: Viti-Levu, Vanua-Levu, Kandavu e Taviuni.

Fu scoperto il 5 marzo 1643 dall'olandese Abel Jansen Tasman, la cui romantica passione per la figlia di Van Diemen, Governatore di Giava, promosse quelle arditissime imprese nautiche, che lo collocarono tra i navigatori più celebri dei suoi tempi, e resero tanto famoso il suo nome. Si crede che Tasman penetrasse nell'arcipelago dal lato di Levante fra il $16^{\circ}$ ed il $17^{\circ}$ grado di latitudine Sud, e non trovandovi alcun ancoraggio, risalisse al Nord, in mezzo alle isole ed ai banchi circostanti alle coste di 
Taviuni e Vanua-Levu. Minacciato ad ogni istante di naufragio, egli si apri infine una via libera dirigendo sopra Undu e Tikambia. Chiamò l'arcipelago delle Figi Prins Willhem Eylandese. Poco più di un secolo dopo, il Capitano Cook passava, di notte, nelle vicinanze dell'isola Vatoa e delle Tartarughe. Il Capitano Bligh del Bounty, nel suo memorabile viaggio in lancia, in seguito all'ammutinamento del suo equipaggio, scorse, nel 1789, una parte dell'arcipelago, e lo rivide, con miglior agio, nel 1792, al comando della Providence. Si dice che il Capitano Wilson, della nave Duff della Missione inglese, nel 1797, avesse voluto seguire la traccia di Tasman, ma meno di lui fortunato, investì sulla scogliera di Nukibasanga.

La storia tace dei primitivi contatti fra europei e figiani; non è peró improbabile, che alcuni dei navigatori del xvII secolo, veleggiando dalle coste Ovest dell'America, abbiano visitato l'arcipelago. Notizie certe e ben precise sulle Figi ed i suoi abitanti si ebbero soltanto al principio di questo secolo. Esse concernono fatti singolarissimi che il reverendo Thomas William, nella sua pregevole opera Figi and Figians, descrive così:

«L'anno 1804 alcuni condannati, evasi dalla colonia penitenziaria di New South Wales, approdarono nelle isole Figi e vi fissarono la loro dimora. Vivevano quasi tutti a Mbau od a Rewa, città i cui Capi concedevano loro grandi favori, ricevendone come compenso il loro aiuto personale, in guerra. Apparvero, in breve, terribili agli occhi degli indigeni, attoniti degli effetti micidiali delle loro armi da fuoco.

«I Capi ostili, vedendo cadere in battaglia i loro migliori guerrieri, senza alcuna causa apparente, credevano che i nemicí bianchi fossero esseri sovrumani, contro i quali fosse inutile lottare, essendo essi vittoriosi sempre e spargendo il terrore e la distruzione ovunque. Codesti malvagi, che non avevano pensato mai a trarre partito ragionevole della posizione acquistata presso i figiani, nutrirono un giorno il desiderio di diventare i padroni assoluti di tutto l'arcipelago; ma inetti per le grandi imprese, 


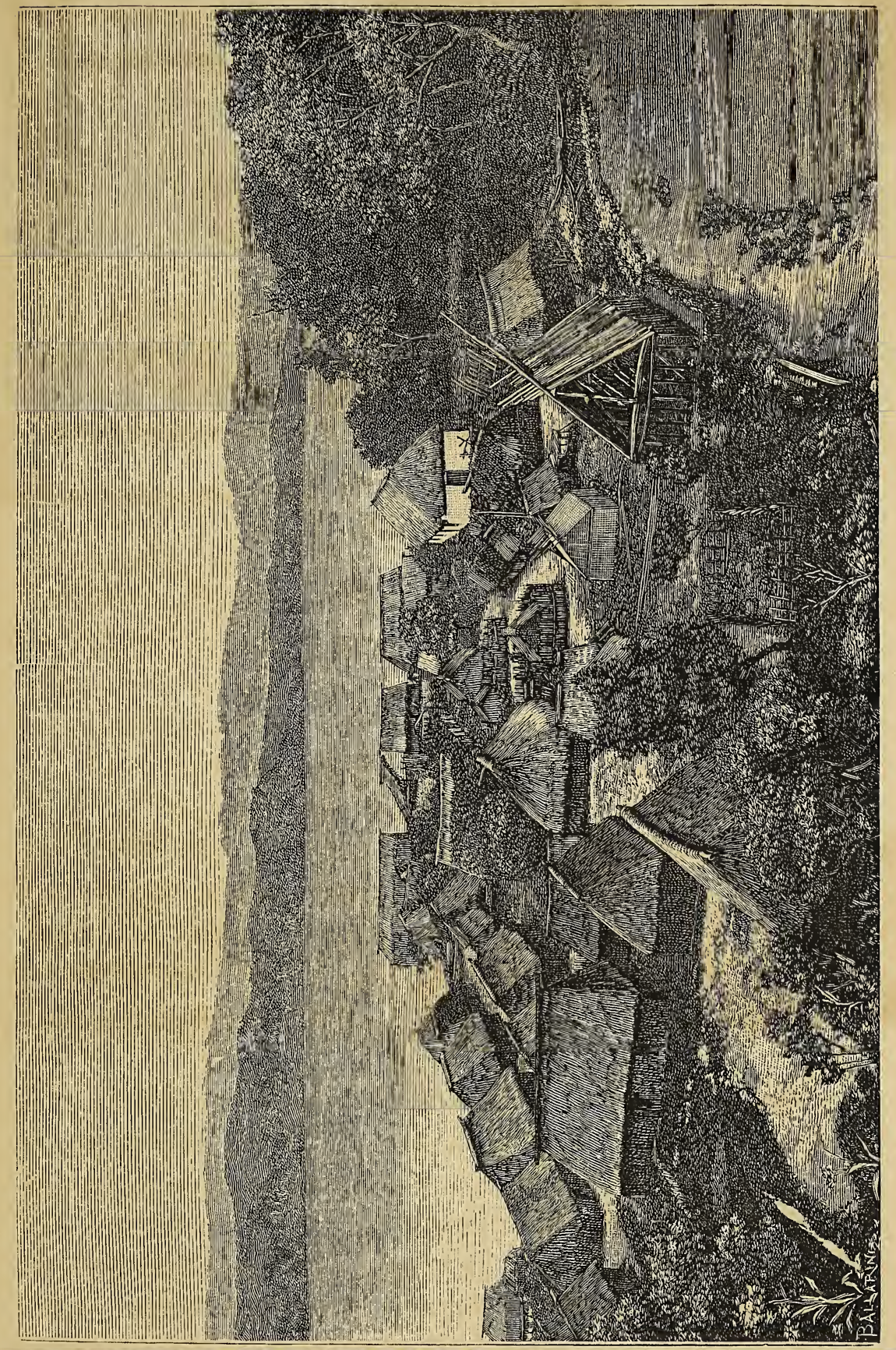

ב 

non seppero sollevarsi mai al disopra di un'esistenza meschina, limitata ai piaceri della maggiore depravazione. Alcuni di essi erano uomini d' inaudita perversità, considerati come mostri financo dai feroci cannibali a' quali si erano associati. Arrivati nelle Figi in numero di 27, la loro schiera non tardò ad assottigliarsi, per effetto delle guerre indigene ed in conseguenza di mortali risse casalinghe. Uno di loro, d'origine svedese, chiamato Savage, il cui carattere manifestava ogni tanto tratti meno abietti, e che era riconosciuto quale Capo della masnada, fu nel 1813 strangolato e mangiato dagli indigeni di Weilea. Due soli erano i superstiti nel 1824, ed uno solo nel 1840. Quest'ultimo, un irlandese di nome Connor, rimase intimo del Re di Rewa, come il Savage lo era stato del Re di Mbau. L'ascendente di Connor sugli indigeni era cosi potente, che ogni sua brama, fosse pure la piu feroce, veniva subito soddisfatta, il Re di Rewa nulla sapendo negare alla sua volontá. S' egli chiedeva la morte di un indigeno, il Re si affrettava ad inviarglielo con un carnefice, affinchè la vittima fosse, in sua presenza, messa nel forno rovente, e poi da lui mangiata. Morto il Re di Rewa, lo sciagurato abbandonó Rewa. Egli era diventato un vero cannibale, e depravato tanto, che i residenti bianchi dell' isola ne avevano gran paura e se ne tenevano discosti. Sul fine della sua vita si dedicó esclusivamente ad allevare maiali e pollame, in mezzo a numerosa figliolanza: cinquanta figli!!»

Mercè l'influenza di Connor, Rewa e poi Mbau assunsero una incontestabile superiorità su tutte le altre isole dell'Arcipelago.

Verso quell'epoca, le voci vaghe ma allettanti, che correvano, in Australia, sulle prodigiose ricchezze dell' arcipelago figiano, consistenti in generi ricercatissimi sui mercati chinesi, legno sandalo, bîche de mer (tripang, oloturia), tartaruga, spronarono alcuni speculatori a recarsi nell' arcipelago stesso. Si armarono apposite navi, e queste trovarono largamente rimuneratore il commercio intrapreso. Certo, i pericoli erano frequenti, e richiedevasi audacia ed intrepidezza per affrontarli; bisognava scendere 
a terra in molti e sempre armati; si naufragava facilmente, ed alcune volte gli equipaggi erano massacrati. Oggi ancora si vedono in alcune città figiane ancore e cannoni antiquati, silenziosi testimoni di passati eventi selvaggi. Ma quante sanguinose rappresaglie di bianchi su quelle coste! Gli episodi di esse, narrati da vecchi trafficanti, trasportano la mente a' tempi più nefasti delle scorrerie di Dampier e di Drake!

La popolazione bianca aumentó lentamente, e nel 1835, grazie alle benevoli disposizioni d'animo dei Capi indigeni, potè fondarsi a Levuka una piccola colonia, i membri della quale esercitavano i mestieri di falegname, di fabbro ferraio, di maestro d'ascia, e nello stesso tempo il commercio.

La scoperta dell'oro in California, nel 1851, diede ragguardevole impulso al traffico figiano con Sydney, Melbourne e California, e vari minatori, che avevano veduto le Figi traversando il Pacifico, vi tornarono per istabilirvisi, quando non era più così facile il fare rapida fortuna alle miniere aurifere. Dieci anni dopo, cioè nel 1861, la popolazione bianca ascendeva a 166 adulti, la maggior parte dei quali altamente rispettabili.

L' ultimo censimento, eseguito nel 1881, faceva ascendere il totale dei coloni bianchi a 2293.

II.

Il figiano e la sua presunta origine.

Il figiano la cui storia non è meno oscura di quella degli altri abitatori dell'Oceania, segnerebbe nell'ordine antropologico la linea di separazione fra le due famiglie papuasica e polinesiana, alle quali tali abitatori appartengono.

Lo si considera comunemente come un ramo dello stipite negro-orientale (negritos), attesa la stretta affinità che sembra 


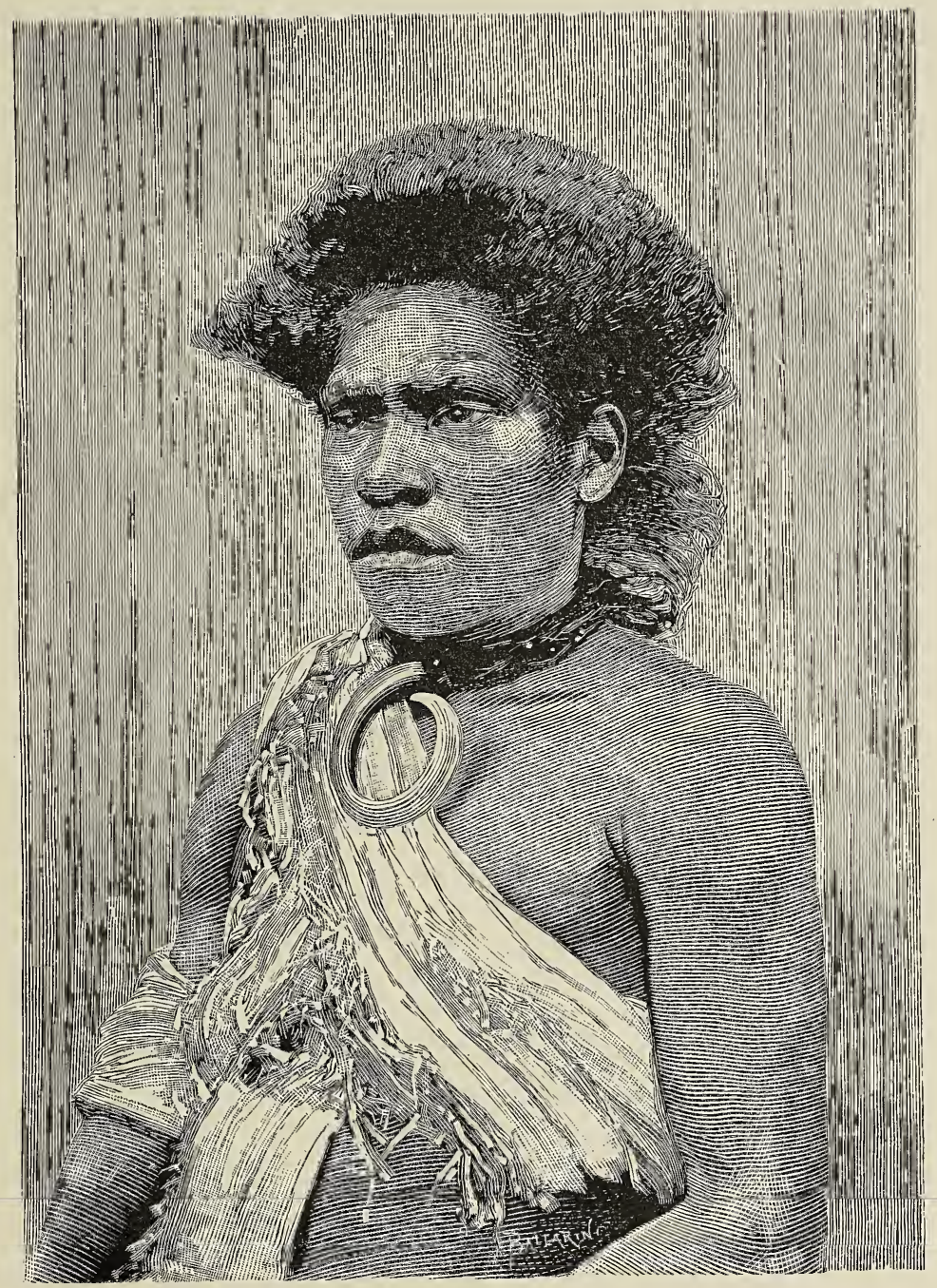

Uomo del litorale. 

avere con gli aborigeni dell'Ultra-India, della Malaia, delle isole di Luzon, di Sumatra e delle altre, che si stendono sino al Giappone.

Si è supposto che codesto stipite, in tempi remotissimi, popolasse un vasto continente, il Limuriano, che avrebbe compreso tutte le coste dell'Oceano Indiano, dal Madagascar sino alle terre piủ orientali del Pacifico, e si sarebbe infranto sotto l'azione di graduali convulsioni geologiche.

I Macedoni, narrano gli storici, furono sorpresi d'incontrare nel corso della loro marcia verso Idaspe, ${ }^{1}$ neri privi di capelli increspati, e Tolomeo, nella sua mappa dell'Aureo-Chersoneso di Malaia (la penisola malese), accennò ad una contrada di neri mangiatori di pesci.

La vecchia razza indo-africana scomparve, per cedere il posto alla razza asiatico-orientale che, a sua volta, subi gl'influssi dell'emigrazione ariana, ed originò il malese-polinesiano. I negri da' capelli ricciuti erano stati scacciati dall'India assai prima che i Macedoni vi apparissero, ma nella fuga alcuni probabilmente riuscirono a rifugiarsi in reconditi siti, sopravvivendo così alle vicende varie del grande impero indiano.

Tuttodi si rintracciano, nel Madagascar ed in alcune isole dell'arcipelago di Tuamotú, le loro orme, e qualche avanzo di loro si trova anche a Sumatra, Celebes, Ceram, Burò ed altre isole malesi.

Il padre Favre dice ch'essi hanno la pelle nera, con i capelli ricciuti, senza l'increspamento, che caratterizza il cafro. L' uomo del suolo: l'Orang Binua; l'uomo dei monti, l' Orang Bulik, furono veduti dal bianco moderno; ed il padre Bourien nel farne menzione, afferma che mangiano di ogni cosa, hanno capelli ricciuti, emettono un forte odore, ed asseverano la verità.

Il negro forse occupó un tempo anche la Nuova Zelanda, e quando il vecchio continente australe, sua patria, sprofondando negli abissi del mare, seppellì la sua culla, egli insieme con gli

${ }^{1}$ Idaspe, ossia Hydaspes degli antichi, Djelem dei moderni: fiume dell'India N. O., proveniente dai monti Imaus. 
Epicornis, Dinormis, Casuarius Bennettii e simili corridori, rimase disperso ed isolato sulle cime degli alti monti.

Ipotesi diverse si fecero intorno alle prime migrazioni di questa antichissima razza; peró, ove si pensi ai molti dubbî che annebbiano il passato di uno dei suoi piu recenti rami: il malese-polinesiano, il mistero che avvolge le sue origini non sorprende piu. Ma, se vi furono migrazioni malesiane, esse precedettero certamente la scomparsa del gran continente australe, e se non è fallace l'asserzione che questo continente medesimo fioriva mentre la maggior parte dell'Europa, dell'Asia e dell'Africa settentrionale trovavasi ancora inondata dalle acque, conviene attribuire alla razza nero-orientale una sterminata antichità.

Il figiano deriva incontestabilmente da un ramo direrso dell'indigeno dell' isola di Tonga, il quale gli sta pur vicino, e di tutti quelli che parlano il polinesiano, imperocchè pochi vocaboli del suo idioma sono affini con la lingua polinesiana: Kalou (Dio), Leva (donna), Siego (Sole), Tolatola (omero), tala (gamba), ecc. non hanno riscontro nel pretto polinesiano, mentre lo hanno in qualche dialetto malese. Bulanò o Bulau, parola usata dal figiano per nominare la luna, appartiene alla lingua malese. A Figi e nel Madagascar sono comuni i nomi dell'albero del cocco, dell'igname, della banana, dell'arum esculento e di altri tuberi e frutti. La $\mathrm{Ca}$ suarina, nell'una e nell'altra località, vien chiamata filau o velau.

III.

\section{Mitologia - Religione.}

Nelle Figi il primo concetto astratto fu il culto degli antenati; ogni isola, ogni localitá ebbe i suoi dei, le sue superstizioni; ogni individuo le sue particolari preferenze, per gli uni e le altre, ed in tanta confusione rimase vaga, incerta la mitologia figiana. 


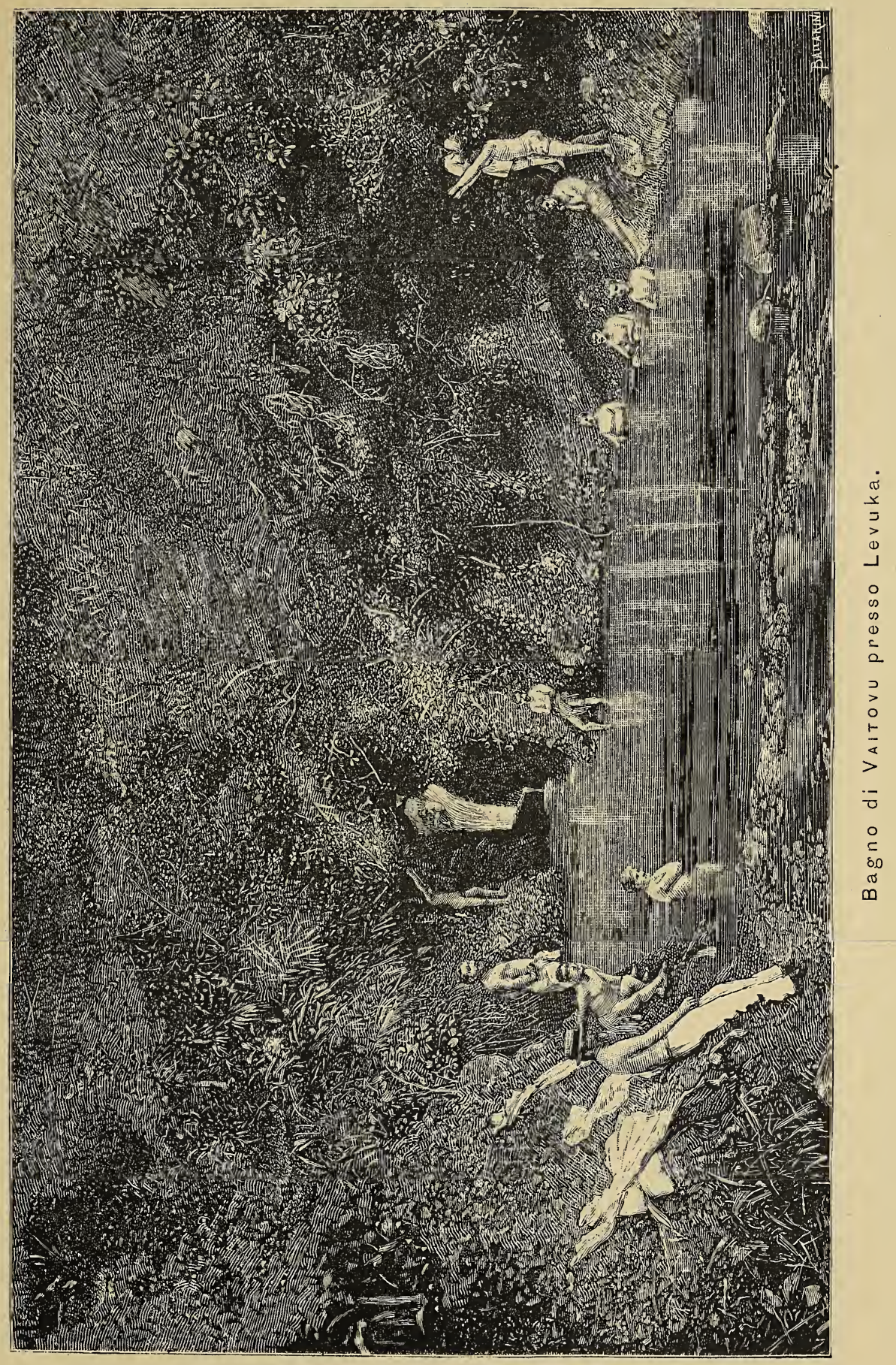



Ndnengei era l' ente impersonale, l'idea mistica dell'esistenza infinita, e nel contempo il dio dell'ira.

Kalou-vu fu il nome delle divinità immortali, Kalou-yalo degli uomini deificati, e pertanto delle divinita soggette a tutte le passioni umane. Simbolo dell'eternita, il serpente.

La mitologia registrava un dio ricercatore di vittime, un dio assassino, un dio destro, un dio prudente, un dio rapitore, ecc., deità tutte ghiottissime di carne umana.

La tradizione lascia sospettare un'origine ebraica facendoci sapere come il mare inondasse improvvisamente la terra (l'isola Viti-Levu) e facesse perire tutti gli uomini, ad eccezione di alcuni che si salvarono in una grande canoa, ed altri, che tessevano una rete da pesca yaka, sulla vetta di un monte rimasto emerso. La canoa era stata costrutta assai prima dell'inondazione, ed aveva schiacciato nel varo, con il proprio peso, molta gente.

A tessere la rete eranvi soltanto donne e fanciulli, i quali in breve furono stretti dalla sete, l'acqua essendo tutta salata; ma venne una persona, che comandò a tutti di seguirlo; ubbidirono, e, giunti presso una roccia, quella persona toccó con una bacchetta la roccia stessa e ne scaturì acqua dolce. Quando le acque si ritirarono, i superstiti erano otto, ed in piccolissimo numero gli animali rimasti vivi.

L'isola Mbenga, al sud di Viti, fu ritenuta come il luogo di approdo della grande canoa, e luogo inoltre in cui, per la prima volta, il dio principale apparve.

Grazie a quest'ultima circostanza, i Capi di Mbenga godevano di una certa preminenza di onori sugli altri Capi dell'arcipelago.

Alberi e piante sacre, come nel culto druidico, ed alcune volte monoliti sacri, segnavano la dimora degli dei. Pretendevasi che nei pressi di Mbau, abitazione di una dea, uno di questi monoliti dasse alla luce una piccola pietra, allorchè una principessa del sangue era relegata nella capitale figiana.

Nessun villagggio senza tempio, e nessuna comunità organizzata priva del suo sacerdote. 
Gli dei avevano speciali ministri, e speciali tempî bene costrutti e riccamente addobbati, con stoffe di color rosso o nero. I tempî erano sollevati dal suolo vari metri, riposando sopra semplici o doppie piattaforme di pietre; vi si accedeva mediante solide tavole di legno. Il principio e la fine della costruzione di un tempio festeggiavansi con sacrifizî e pasti di carne umana.

Il prete ambati, personaggio di molta importanza, divinatore, ispirato, comunicava ai Capi ed al popolo riuniti i responsi degli oracoli evocati, in mezzo ad eccitamenti nervosi, ch' egli aveva avuto l'arte di provocare. Preti ed anziani consumavano le offerte, in vivande, fatte agli dei, offerte indispensabili per ottenerne i favori.

Il figiano credeva fermamente ad un'esistenza futura, durante la quale doveva ricrearsi con canoe ed armi, e cibarsi di frutti d'ogni specie. Non fu mai idolatra, nel vero senso della parola, sebbene immaginasse, che taluni uccelli o pesci fossero il ricettacolo dei suoi dei e per quegli animali avesse particolare venerazione.

Immaginavano che, morti, l'anima si sarebbe separata dal corpo, per andare a Mbenga, e sottostarvi a giudizio divino: ma essa doveva prima combattere con un gigante ed uscire illesa dalla tenzone, chè altrimenti era condannata a vagare eternamente nella montagna.

L'anima doveva occupare nell'altra vita un posto, per dignità, corrispondente alla violenza della morte, onde i vecchi si suicidavano o i figli si facevano i carnefici dei padri.

Secondo la leggenda, l'uomo nacque dall'incubazione di due uova di falchetto, operata dal dio Ndnengei, della cui bella apparenza si era invaghito. Aveva Ndnengei deposte le due creature, frutto dell' incubazione, ai piedi di un grande albero, da lati opposti, ove rimasero immobili fino all'età di sei anni, nella quale il maschio mossosi, scoprì la sua compagna e le disse: Ndnengei ci ha creati perchè popoliamo la terra.

Un'unica origine aveva adunque per i figiani il genere umano: primo a nascere sarebbe stato il figiano che, perverso, divenne nero 


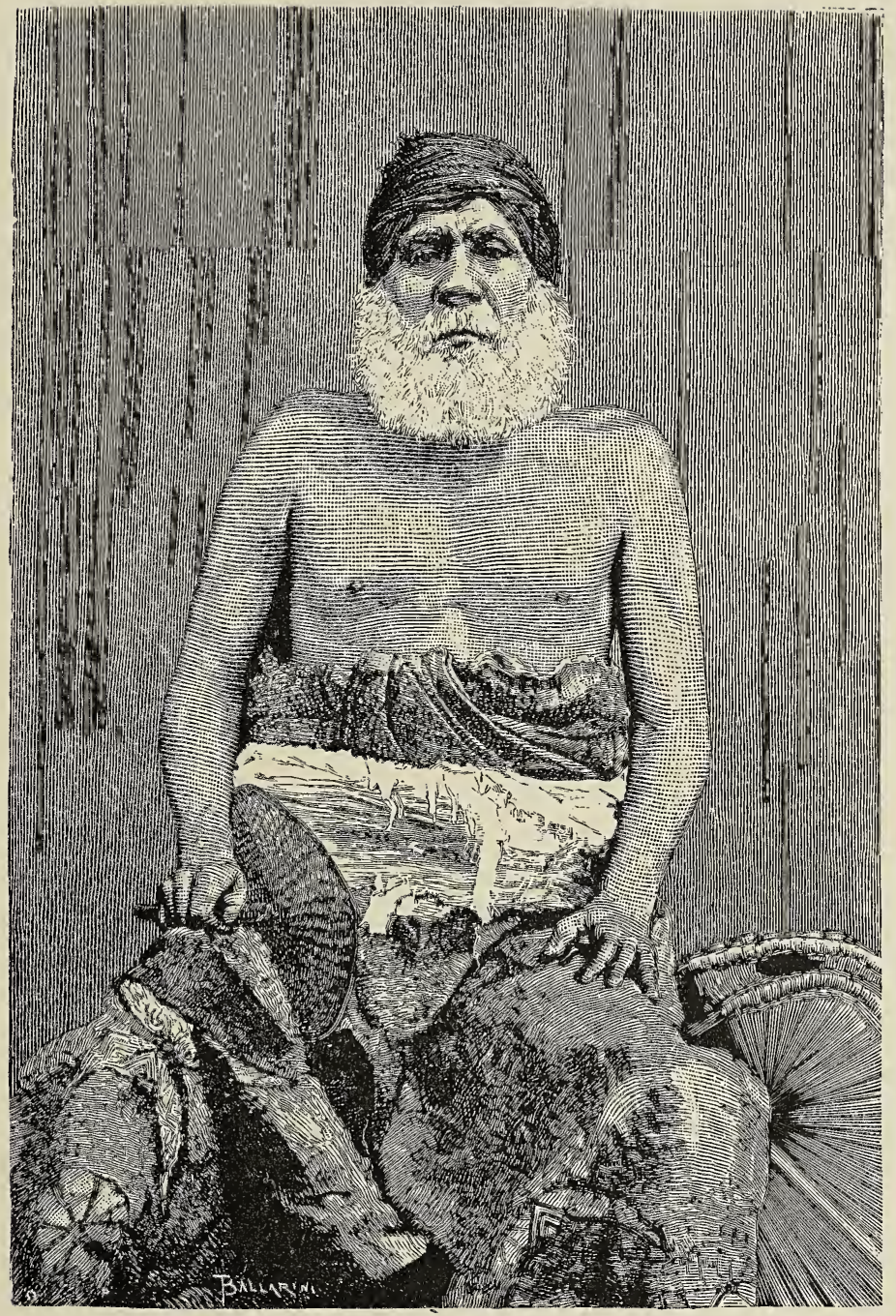

Thokarnbau, re dei Cannibali. 

e ricevè scarsi indumenti; nacque dopo il tonga, meno cattivo, meno nero e meglio vestito; il Papalagis - l'uomo bianco - ultimo a nascere, non avrebbe peccato ed avrebbe avuto molti indumenti.

L'istituzione del tapir o tabir o tambir, propria della Polinesia esisteva anche presso i figiani. La persona dei Capi, gli oggetti da loro adoperati erano tapú, ossia sacri. Al pari degli ebrei e dei polinesiani osservavano il rito della circoncisione, praticandola sopra i ragazzi dell'etá di sette od otto anni. Un pezzo di bambú affilato serviva d'istrumento da taglio, e la guarigione si effettuava rapidamente. Compievasi la circoncisione sopra dieci o venti maschi alla volta, e questi, per parecchi giorni, abitavano insieme in un fabbricato pubblico, ricevendo giornalmente il vitto - legumi cotti - da donne, che nel portarlo intuonavano canzoni di circostanza. La morte di un Capo era occasione prescelta per celebrare la circoncisione.

Le donne - non mai gli uomini - dovevano, per comando di Ndnengei, tatuarsi alcune parti del corpo, coperte dal likir; ${ }^{1}$ l'opposto di quanto usasi nelle isole Tonga, ove gli uomini solamente si tatuano.

Fra i riti più radicati, nella società figiana, annoveravasi quello del soró: espiazione, perdono, offerta di pace, che mirava a raffermare le relazioni di amicizia, ovvero ad impietosire il nemico. Quando offerivasi un dente di balena od una clava, il sorò si riferiva alle appropriazioni indebite, dall' igname di nessun valore al ratto della moglie di un Capo; con la presentazione di una canna, il sorò riguardava colpe maggiori; ed il sorò implicava umiliazione profonda se consistente nel dono di una lancia. Il soró piu solenne, il sorò per i delitti di sangue, richiedeva che il supplicante si cuoprisse il petto e le braccia di ceneri, imitando così, in qualche guisa, l'antica consuetudine dei penitenti cattolici, simboleggiata oggi dalla cerimonia del primo giorno di quaresima.

${ }^{1}$ Il tikù figiano corrisponde al paraù tahitiano, che è l'oggetto con cui gli indigeni si cingono i fianchi. 
IV.

Indole del figiano; suoi antichi costumi e leggi.

Il figiano ha piacevole aspetto; è robusto, proporzionato e di bella statura. Il colore della pelle è cioccolatte con sfumature chiare, s'egli vive sul litorale, ed oscure s'egli è invece montanaro; ne' due casi le tinte sono sempre piu sbiadite per quanto più egli è elevato nella gerarchia della propria tribù. Naso schiacciato alquanto, bocca alquanto larga, con i denti bianchissimi, capelli ricciuti e fini di color nero ed anche naturalmente fulvo. Incalcina la sua foltissima capigliatura, che prende allora questa ultima tinta. Si dipinge di rosso o di nero cupissimo, ed acconcia i. suoi capelli a ventaglio, in mille modi diversi, per vezzo o per incutere timore.

Sulla sua indole molti e disparati giudizi furono portati. Taluno lo descrisse pieno di contraddizioni: or buono e trattabile, or crudele ed ostinato; di poco o nessun coraggio, nemico implacabile, ingrato, pigro e traditore, e pur tuttavia malleabile con tatto e fermezza. Altri sostennero ch'era esagerata ed anche ingiusta questa descrizione, opponendo il fatto che il figiano, nel seguire i suoi capi, in guerra, ovvero i bianchi condottieri delle sue tribù, diede, in ogni occasione, prove irrefragabili di lealtà, fedeltà e valore. Infine, parecchi europei, che vissero lungamente in mezzo ai figiani, affermarono, che per amabilità e bontà d'animo nessuna gente di colore poteva superarli, e che in nessun altro paese, posto nelle stesse condizioni d' isolamento delle Figi, rispetto ai paesi civili e forti, i bianchi avrebbero potuto trovare cotanta ospitalità.

Come, in generale, tutti i popoli di colore, i figiani non hanno personalità; fa mestieri dirigerli e sorreggerli in ogni cosa; senza 
un Capo, che li guidi costantemente, sono incapaci di agire. Grandi fanciulli e nulla più

Sono, nel loro genere, industriosi, ma non amano il lavoro sostenuto e faticoso, del quale peraltro la provida natura non fa sentir loro il bisogno, e si mostrano insensibili agli stimoli maggiori, per cui il bianco sa fare prodigi di attivita e di energia: l'amore e l'ambizione.

Manifestarono sempre un certo qual gusto artistico nella fabbricazione delle armi - di legno tutte -: lance, mazze o clave, ascie, ecc., e nella ceramica, che producevano senza ricorrere a forme, adoperando argilla, cuocendola al sole ed inverniciandola con resine.

Ebbero fama di eccelsi costruttori di canoe, alcune delle quali potevano trasportare, sulla propria piattaforma, quattrocento e più guerrieri.

La musica e la danza figurarono ognora fra i loro passatempi prediletti; la prima, meno melodiosa della tahitiana, si distingueva essa pure per la perfetta cadenza; e la seconda, piu corretta e pudica dell'upaupà, ricordava leggende guerriere ed avvenimenti storici nativi, mercè una mimica espressiva di gesti, contorsioni, inchini, giri e battimani.

Principali istrumenti musicali erano i corni - conchiglie di mare -, i grandi e piccoli tamburi - bambi spaccato o tronco di albero di cocco scavato -, i flauti nasali, le zampogne.

Ricercavano i giuochi atletici, nuoto, lancio di pietre, salti, lotte corpo a corpo, ecc. Ancora adesso sono gelosi osservatori dell'etichetta, massime verso i loro Capi: ubbidiscono ossequenti a' loro ordini, applaudiscono ad ogni loro parola con battimani o scoppiettio di lingua, entrano prostrati nelle loro abitazioni, e non si fanno mai lecito di passare alle loro spalle quando si incontrano con essi. Il saluto figiano è lo starnuto.

Molto sobrî, base del loro nutrimento furono e sono i vegetabili : tarò, igname; i frutti : cocco, esculenta arum, banana; ed i pesci. La cucina figiana non difettó mai di ricercatezza, constando 
di una dozzina di varietá di pani, di diverse salse e zuppe e di molti dolci. Fecero sempre cuocere le vivande, mangiando peró crudi alcuni pesci e molluschi freschissimi. L'uso di bibite calde - infusioni di piante aromatiche e foglie - è vecchissimo e perdura. Bevanda comune, l'acqua del cocco o meglio l'acqua pura. Prendevano il kava, chiamato yagona, e lo prendono con solennità; si asserisce da anziani indigeni, che un tempo grattugiavano, per prepararlo, la radice del piper methysticum, anzichè masticarla come fanno presentemente ad imitazione de'polinesiani. ${ }^{1}$

I bambini de' due sessi andavano nudi oltre l'età di 10 anni, e se figli di Capi, durante maggior tempo. Gli uomini avviluppavano le gambe ed i fianchi con il masi, specie di fascia filamentosa, lavorata in paese, di lunghezza variabile fra i due e gli ottanta metri, in ragione dell'importanza dell'individuo. Le estremità della lunghissima fascia pendevano a forma di strascico. I dignitari portavano un gran turbante. Le donne cingevano il liki, fascia a frange, allacciata dal lato destro e munita di strascico ne' dì festivi.

Cannibali, per ubbidienza religiosa i più, ed i meno per appetito della gola, cessarono recentemente di esserlo.

Codesta singolare tendenza all'antropofagia, che spicca tanto in mezzo della gente oceanica, e che è così contraria alle naturali leggi della propagazione della specie, dovrebbe, a nostro credere, rivelare un'antica imperiosa necessita, che, mutatasi in ragione di Stato, degenerò in ghiottoneria, per i potenti, in forza dell'abitudine.

Forse un'improvvisa quanto smisurata sproporzione fra gli abitatori e le risorse alimentari del suolo, rispondente all'epoca de' grandi mutamenti geologici australasiani, determinó gli orrori della fame in quelle masse umane, e spezzando ogni legame sociale, armò disordinatamente il più forte contro il piu debole, finchè non si ristabilì l'equilibrio fra consumatori e produzione. Da ciò

1 Vedi Tahiti, cap. VIII, pag. 99, per la preparazione del kava. 


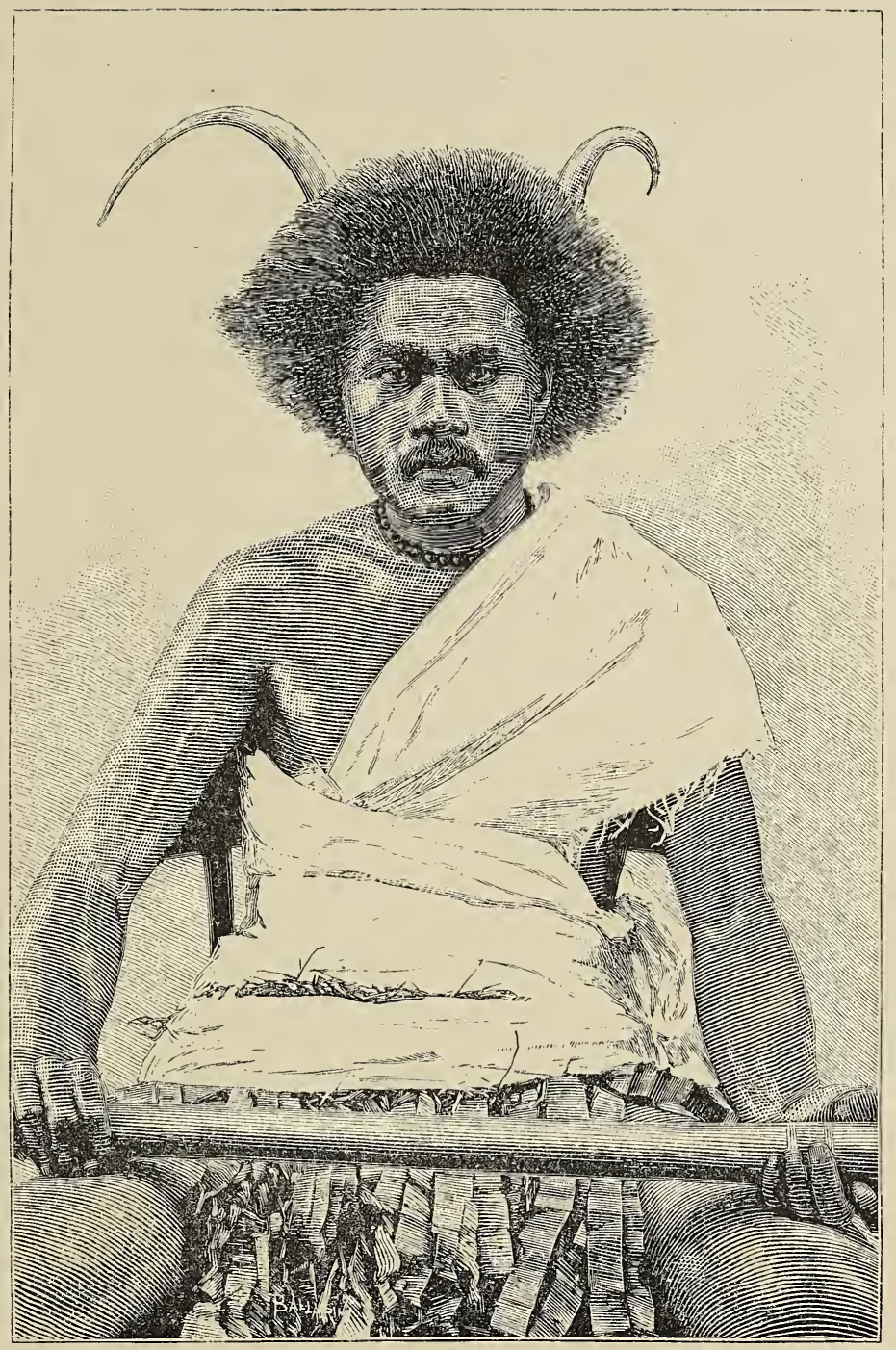

Figlio del Foko-Tui Kadavu. 

il cibo umano! In seguito, il conflitto divenne meno tenace, la violenza della lotta si attutì, e riapparve la società. Rimasero però le influenze dell'eredità, che mantennero il costume antropofagico sopra scala minore, ma con crudeltà vieppiu raffinata. ${ }^{1}$

Negli ultimi anni, naufraghi e prigionieri di guerra fornivano il maggior contingente a codesti pasti nefandi, in cui la vittima, unta d'olio ed avvolta in foglie, veniva posta viva in un forno, sopra incandescenti ceneri, e ricoperta di pietre infuocate.

Strana incoerenza! la generalitá de'figiani provava estrema ripugnanza a mangiare carne umana, al punto di respingere dalla mensa quella di tartaruga che, filamentosa, aveva tutte le apparenze di quella. ${ }^{2}$ Non si osava toccare la carne umana con le mani, sicchè usavansi, per mangiarla, apposite forchette, dopo averla deposta in deschi appositi, le une e gli altri ritenuti tabu, ossia esclusi da qualsiasi altro uso.

I figiani furono reputati da' loro vicini, i tonga, arditi navigatori. Possedevano alcune nozioni di astronomia; dividevano l'anno in lunazioni, e per correggere la differenza tra l'anno lunare e l'anno solare, pare assegnassero un lungo periodo ad uno degli 11 mesi del proprio anno, al valu $i$ werewere (mese schiarente). Diversamente da'melanesiani delle Nuove Ebridi, i figiani costrussero sempre abitazioni comode, case con la base staccata dal suolo. Le loro città dell' interno erano quasi tutte circondate da parapetti e fossi colmi d'acqua; in guerra, per maggior precauzione, gli abitanti formavano nuove difese, e durante la notte si ritiravano, dalla cittá, rimanendo a portata delle prime linee di attacco.

Le leggi figiane erano in parte patriarcali ed in parte feudali; ma la piu importante di tutte, quella che in certo modo strin-

${ }^{1}$ Poco prima delloccupazione inglese, alcuni Capi, per arricchire la propria mensa, solevano ordinare l'uccisione di quel loro suddito, che più solleticava il loro palato.

${ }^{2}$ La tartaruga era però cibo regale, e la pesca di essa si faceva con grande solennità. 
geva in un fascio le sociali e politiche istituzioni, era la legge lala, che equivale al diritto di esazione rispetto alle persone ed alle cose. Siffatta legge, in vigore da tempo immemorabile, fu il baluardo della mitologia figiana, la base di ogni relazione fra Capi e popolo, la espressione dell'autorità e del prestigio di quelli, de'privilegi e della sottomissione di questo.

Quando un Capo, con fini personali, ovvero nell' interesse pubblico, esigeva l'esecuzione di un lavoro qualsiasi, come la costruzione di case o canoe, la coltivazione di campi, la pesca, ecc., egli invocava la lala, per reclutare le braccia necessarie.

Allora, la gente designata si recava immediatamente sui luoghi, per compiere, sotto la di lui direzione, il lavoro ordinato. In tale occasione, egli organizzava feste in onore de' suoi ospiti, i lavoratori, e li colmava di doni, ch'essi ricambiavano con altri doni.

Le colpe commesse a danno dell'autorità o del pubblico implicavano, in forza della lala, la solidarietà, a seconda de' casi, di tutto un villaggio, città o tribù, e quindi l'estensione a quello o a queste del castigo.

La lala regolatrice, in pace, dell'ordine e del funzionamento economico del paese, presiedeva, in guerra, all'organizzazione militare 'de' combattenti.

Sventuratamente, questa legge, in armonia con le inclinazioni ed i bisogni del popolo, spesso si trasformó in istrumento di tirannide e di oppressione, nelle mani di capi brutali o deboli.

L'unità sociale era il mataqali - la famiglia -; la riunione di varie famiglie formava il quali o qali - la gente -; varî qali costituivano finalmente il matanitic - la tribù.

Ognuna di queste agglomerazioni aveva un Capo eletto dai componenti l'agglomerazione medesima; Capi e popolo erano i padroni ereditari (aitaukei) delle terre da loro occupate ed il diritto di proprietà del suolo si estendeva cumulativamente al Capo, alla famiglia ed all' individuo. Tranne i Capi di vasti territori, nessun singolo individuo poteva vendere terreni.

Le guerre, sia per il conseguente bisogno di accrescere i 
mezzi di attacco e di difesa, sia per forza di conquista, imposero a varie tribí un Capo comune, un gran Capo o sovrano - Tui che in più d'una occasione assunse il governo di numerosa gente.

I Capi delle piccole isole perdettero, l'uno dopo l'altro, la propria autonomia; rimasero, ne' tempi piủ recenti, a contendersi il predominio dell'isola maggiore, Viti-Levu, e con esso quello dell'intero arcipelago, due soli grandi Capi Thakombau di Mbau e Vasu Raivalita di Rewa.

La societa figiana si divise in sei caste: $1^{a}$ Re e Regine; $2^{a}$ Capi d'isole estese; $3^{\text {a }}$ Capi di cittá, preti, oratori, uomini influenti; $4^{\text {a }}$ guerrieri di bassa estrazione, Capi de' falegnami, Capi de' pescatori di tartarughe; $5^{a}$ plebe; $6^{a}$ schiavi di guerra.

Riguardo a gerarchia politica essa annoverava: il Tui-Re o Capo principale -; il Roko-Tui - personaggio piu elevato in grado dopo il Re -; il Vu-Ni-Valu - membro della famiglia reale, ovvero Re -; il Buli - l'eletto a cariche elevate -; il Matanivanua - proprietario, uomo influente, oratore -; il Taukei - piccolo proprietario -; il Bati - guerriero -; il Talatala - uomo di fiducia dei Capi -; il Talaki - domestico de' Capi -; ed il Mata-messaggiero politico.

Erano poligami i figiani, e comprendevano fra le istituzioni politico-sociali il matrimonio, che celebravasi con cerimonie speciali. La donna non poteva contrarlo senza l'assenso del fratello, tuttochè avesse ottenuto quello de' genitori. Destinata a diventare la schiava del marito, i parenti incaricati di presentarla allo sposo, sulla soglia della sua casa dicevangli: «Prendila, è tua; vendila, uccidila, mangiala, se non ne sei contento ».

Punivasi l'adulterio della moglie con la morte. Al decesso del marito era strangolata, per procurarle la felicità eterna! Incinta, doveva ritirarsi presso i parenti, ed avvenuto il parto, interrompere qualsiasi relazione con il marito, per dedicarsi esclusivamente all'educazione del figliuolo, la quale durava alcuni anni. In questo lasso di tempo la nascita di un nuovo figlio sarebbe stata considerata come gravissima offesa verso i parenti. 
Il numero delle mogli segnava il grado di dignità de' Capi, numero sovente considerevole, che variava fra le 50 e le 100 . Ereditario, per discendenza femminile, il rango sociale, la moglie di piu alto lignaggio aveva preminenza sulle altre, ed i suoi figli soltanto acquistavano diritto alla successione.

Al defunto Capo succedeva il fratello maggiore; a questi il figlio primogenito del defunto, ed in sua vece il figlio primogenito del fratello maggiore.

I nipoti - vasu, se maschi e di famiglia cospicua, godevano di sconfinati privilegi, e tali da porre spesso a repentaglio l'autorità ed il prestigio degli zii.

Codesti costumi, che aizzavano fortemente le passioni della gelosia e della cupidigia, furono, come è facile idearlo, fra le domestiche pareti, fonti perenni di disordini, che si risolvevano spesso in delitti di sangue, tra cui erano comuni gl'infanticidî. 


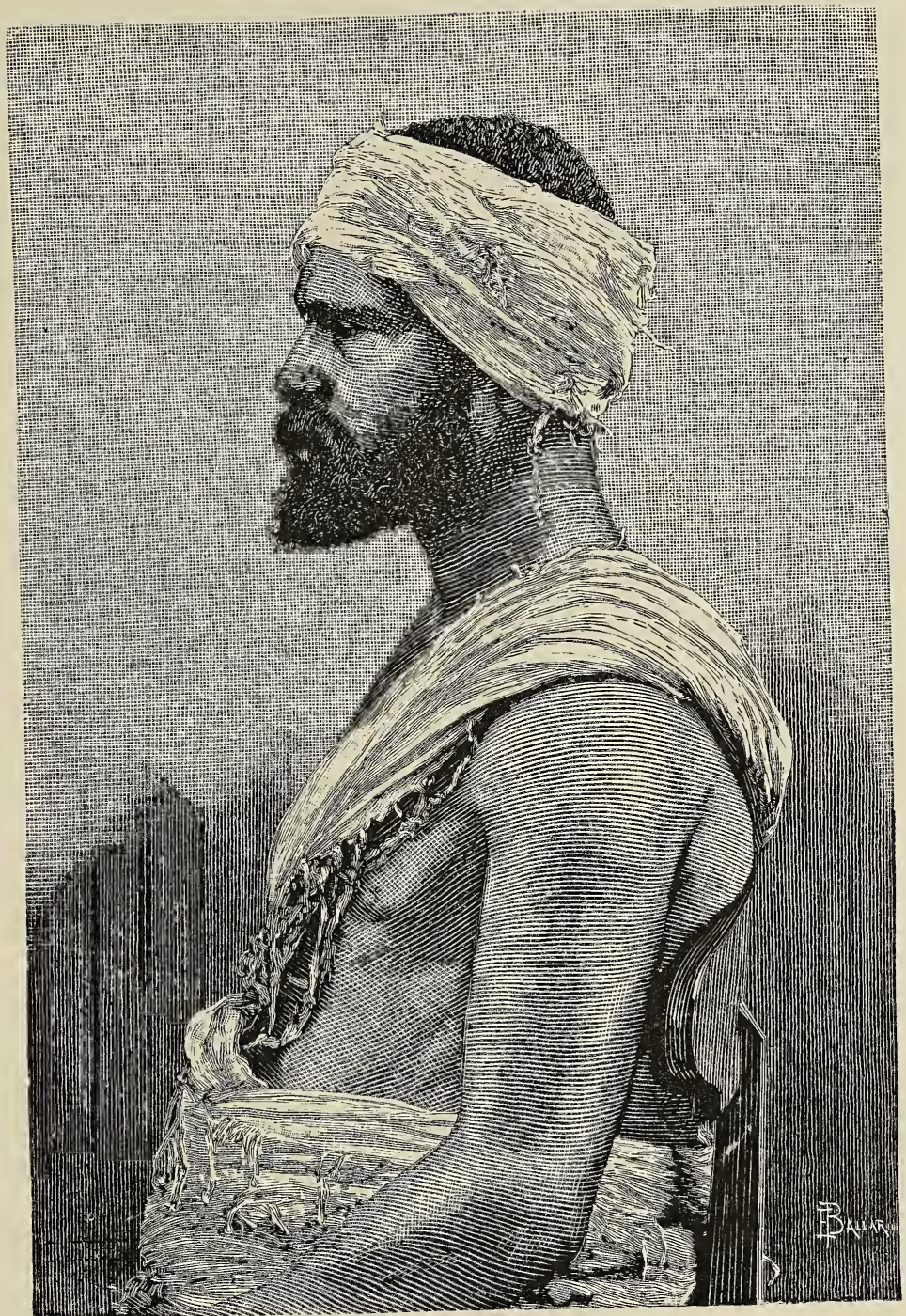

Buli-Tai-Ievu. 



\section{CAPITOLO III.}

\section{ORDINA M E T TOPOLITICO}

\section{I.}

Annessione delle Figi ai dominî britannici.

L'arcipelago delle Figi è oggi possedimento della Corona inglese, la presa di possesso avendo avuto luogo il 24 giugno 1875.

I documenti ufficiali registrano ne' seguenti termini l'atto solenne di adesione del Re e de' Capi figiani, che chiarisce la natura delle prerogative indigene nel governo del paese:

"A mezzodì del 5 dicembre 1877 il Governatore Sir Arthur Hamilton Gordon G. C. M. G. sbarcava dalla sua lancia sul molo di Rewa, attesovi dal Vu-Ni-Valu (Re) e da' Capi indigeni.

«Seguendo una vecchia usanza, gli è stato presentato il veiqalori - cerimonia del benvenuto; in seguito si sono tutti incamminati verso la gran casa del Consiglio, costrutta per l'avvenimento.

«Il Governatore ed il Vu-Ni-Valu entrarono nella cașa del Consiglio per i primi, e, sedutisi, venne preparata la yaqona (kava).

«In seguito, S. E. trasmise a' Capi presenti la risposta di S. M. la Regina alla lettera indirizzatale da' Capi figiani l'anno 
innanzi, invitandoli a pronunziarsi sui quesiti che andava ad enumerare, ed avvertendoli che la loro opinione sarebbe poi stata presa in considerazione dall'assemblea de' Capi:

« $1^{\circ}$ Sul reparto delle terre di una stessa tribú fra i suoi membri;

« $2^{\circ}$ Sui provvedimenti migliori per evitare l'incendio dei boschi;

« $3^{\circ}$ Sull'appianamento de' varì dissensi relativi a confini;

" $4^{\circ}$ Sull'adozione di un regolamento indigeno uniforme, per la custodia, il trattamento e la disciplina de' prigionieri delle diverse provincie.

«Il reverendo A. I. Webb recitò una preghiera, ed il Governatore con il $\mathrm{Vu}-\mathrm{Ni}-\mathrm{Valu}$ si ritirarono lasciando che l'Assemblea svolgesse l'ordine del giorno concernente l'epoca e la durata delle sedute ».

Nel 1852, il Re di Mbao - Thakombau - detronizzato dai ribelli, con i soccorsi del Re di Rewa, suo rivale, chiese, per suggerimento de' missionari wesleyani, l'aiuto di Re Giorgio di Tonga. Questi, alla testa di tremila uomini, che aveva fatto trasportare su grandi canoe, sconfisse il Re di Rewa e ripristinò sul trono Re Thaliombau, il quale, grato a' missionari, abiurò il paganesimo e si fece cristiano. Da quel giorno il Re figiano fece getto degli espedienti di Governo pagani, efficaci tra selvaggi e barbari per dare lustro e podestà ai Capi, abdicando cosi implicitamente la sua indipendenza, e diventando l' istrumento insciente de' missionari. Uomini scaltri, gli wessleyani si mostrarono in quest'occasione degni della loro fama di gente pratica, che posando saldamente i piedi in terra, per poter volgere meglio lo sguardo al Cielo, non disprezza i beni mondani.

$\mathrm{E}$ sia detto per incidente, il missionario protestante inglese possiede l' intuito delle imprese terrestri utili. La famiglia, stimolo al lavoro ed al guadagno, rende intimamente unita, per lui, la coscienza delle necessità della vita all'impero della Bibbia; ascetico, coglie l'opportunitá di dedicarsi alle speculazioni industriali e 


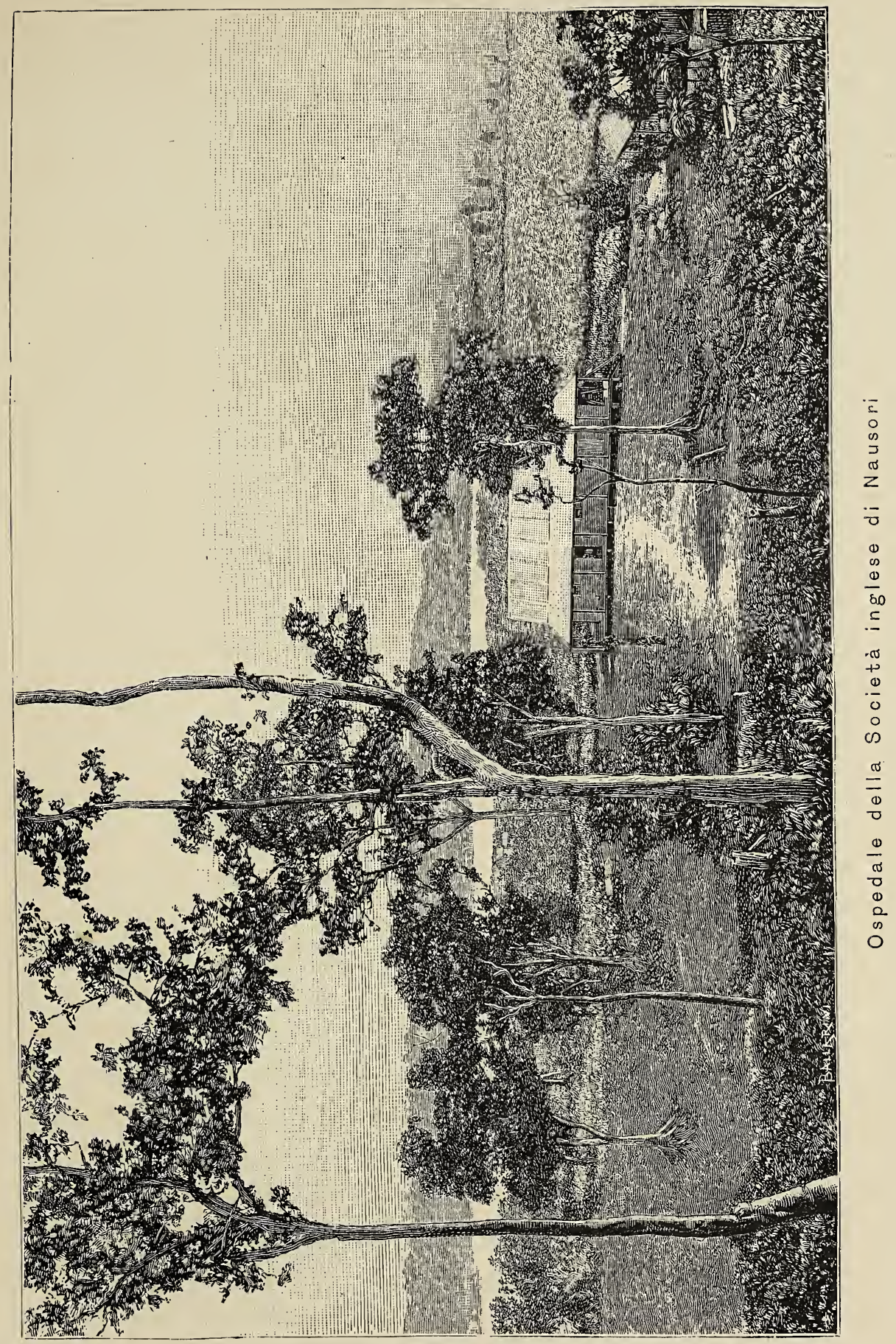



commerciali, pure meritandosi sempre il titolo di pioniere della civiltá, per le vittorie morali e materiali, che, con le virtì dell'abnegazione, del coraggio e della filantropia, sa riportare sui popoli selvaggi o barbari. Il pensiero della famiglia lo mantiene nella cerchia del mondo reale, ove guarda alla patria, a cui la famiglia stessa è legata da tanti vincoli. Egli non dimentica mai di essere un cittadino della Gran Brettagna, che ha il dovere di contribuire, in ogni occasione, alla grandezza e alla prosperitá del proprio paese.

I missionari wesleyani erano dominati dall'idea di costringere Thakombau a rimettere la signoria delle sue terre all' Inghilterra, idea, che, da loro creduta matura nel 1856, manifestarono con replicate ed insistenti istanze al Governo inglese, perchè intervenisse negli affari figiani.

Dietro quelle istanze il Comandante Denhame dell'Herald di S. M. la Regina, intento, nell'Arcipelago, a lavori idrografici richiesti dai frequenti naufragi di navi inglesi, ebbe incarico di riconoscere lo stato vero delle cose. Diede fondo nelle acque di Mbau ed invitò a riunirsi sulla sua nave Re Thakombau, Re Giorgio che non aveva ancora sgombrato, con le sue truppe, il paese ed i missionari wesleyani e cattolici. In questo ritrovo egli mosse a Thakombau varie domande sulla cessione del suo regno all'Inghilterra, le quali non sortirono l'effetto desiderato dagli wesleyani: il Re di Mbau accettava sì il protettorato inglese, non già la sovranitá, e però negava recisamente di voler cedere i suoi dominî. Dinanzi a siffatta negativa, che dissipava ogni dubbio sulle intenzioni del Re delle Figi, il Comandante dell' Herald sciolse la riunione e salpò per ritornare a' suoi lavori scientifici.

Salvata la sua dinastia, Thakombau rimaneva, nonostante, avvinghiato strettamente dagl' interessi varî de' bianchi.

A fianco degli sforzi wesleyani, per accrescere le risorse economiche dell'Inghilterra, serpeggiava con identico scopo, a benefizio però degli Stati Uniti, una corrente di maneggi politici, di cui era anima il fu signor I. B. Williams, Console americano. Fin 
dal 1849 il signor Williams, in seguito all' incendio della sua casa, avvenuto mentre egli festeggiava l'anniversario della Repubblica, per effetto dello sparo d'armi e di razzi, aveva avanzato a Thakombau una domanda d'indennizzazione attribuendo agli indigeni le perdite sofferte. Altri reclami e più incalzanti egli porse successivamente al Sovrano figiaro, per danni arrecati ad una Societá americana, nel saccheggio di due de' suoi magazzini e di una goletta, e per la mancata promessa degl'indigeni di consegnare alla detta Società una certa quantità, pattuita, di olio di cocco, in compenso di somme di denaro anticipate. In ultimo, fece capo al suo Governo, che stimó bene intromettersi nella faccenda, ordinando nel 1855, dopo preliminari investigazioni, al Comandante Boutell della nave John Adam di fare un'inchiesta. Gl' indigeni furono riconosciuti colpevoli e chiamati a pagare, a titolo di risarcimento di danni, la somma di 45,000 dollari, con la minaccia di un'occupazione militare americana, qualora il pagamento non fosse stato eseguito sollecitamente.

Thakombau si trovava nell' impossibilità di soddisfare siffatte esigenze, ma ridotto a dover parare, ad ogni costo, le minaccie fattegli, si risolvè a proporre al Governo inglese di saldare, in sua vece, il debito impostogli dagli Stati Uniti, in cambio della cessione dell'Arcipelago, riservandosi di conservare per sè rango e titolo di Tui-Viti (Re delle Figi).

A tale intento fece redigere uno schema di convenzione, ch'egli ed i Capi dell'arcipelago firmarono, e che il signor Pritchard portò in Inghilterra nel 1859.

La colonia europea delle Figi accolse con favore la risoluzione presa dal $\mathrm{Re}$, e la caldeggiò con tutti i mezzi possibili facendo segnatamente risaltare, fra i vantaggi che il commercio inglese avrebbe tratto dall'accoglienza della proposta figiana, la possibilità di coltivare su larga scala il cotone, scarso in quei tempi, sui mercati inglesi.

Trascorsi alcuni mesi, il Colonnello Smythe, dell'artiglieria reale inglese, giungeva alle Figi in qualità di Commissario della 
Regina, per procedere a più recenti e complete indagini, ed esprimere avviso sulla convenienza o meno di accogliere l'ultima proposta del Re e de' Capi.

Quest'arviso, che non si fece aspettare, fu contrario alla proposta di cessione; e poichè, in quell'epoca, difficoltà d'indole militare, sôrte nella Nuova Zelanda, per opera degli indigeni, destavano gravi preoccupazioni, le autorità imperiali erano poco propense a porsi sulle braccia un altro popolo irrequieto, e l'avviso del Colonnello Smythe trovò subito eco presso di esse, che è dire presso il Gabinetto britannico.

Fallito il tentativo di ottenere dal Governo inglese il denaro reclamato dagli americani, Re Thakombau lo accettò da' privati, da una Societá, cioè, di Melbourne, a cui egli cedè, in premio, le isole di Mbenga e Goro, la baia Natera, nell'isola Venua-Levu, ed altre estensioni di terreno, nel fertilissimo distretto di Suva.

Il Re delle Figi si era in tal guisa liberato dal ginepraio americano, ma ciò poco importava a' coloni bianchi: essi avevano d'uopo di sicurezza, per dedicarsi al lavoro proficuo, e non potendo ottenerla dalle leggi e dai costumi figiani, continuarono ad agitarsi, in cerca di un'organizzazione politica conforme ai loro bisogni. I dissensi, le ostilità de' Capi paralizzarono per lunga pezza i loro sforzi, e non fu che sui primordi del 1871 che Thakombau, assistito dagli europei signori Woods, Sager, Bult, Hennings, riusci a convocare un certo numero di delegati delle due razze, per formulare una Costituzione.

Nell'agosto dello stesso anno que' delegati elaborarono uno Statuto per il Regno delle Figi, insieme con i regolamenti e le condizioni secondo cui il Governo doveva funzionare; indi si separarono, e fu proceduto alle elezioni generali, dalle quali emerse il primo Parlamento figiano.

La forma del Governo sancita comprendeva tre poteri: esecutivo, legislativo e giudiziario; il primo rappresentato dal Re e da' suoi Ministri, il secondo da un Consiglio privato della Corona e da una Camera di Deputati, il terzo da una Corte suprema. Il 
Consiglio privato annoverava tutti i Governatori delle provincie; la Camera de' Deputati era composta dagli eletti da' distretti elettorali; la Corte suprema ${ }^{1}$ dal Capo della Magistratura e da due Giudici, di cui uno indigeno.

Le leggi votate dalla Camera dovevano sottoporsi all'esame del Consiglio privato, che senza avere il diritto di respingerle, non approvandole, doveva rinviarle alla Camera per ulteriori studi.

Il numero de' Deputati era fissato a 40, ed in nessun caso doveva essere inferiore a 20.

Ogni cittadino maschio e maggiorenne acquistava la qualità di elettore, mediante il pagamento di tasse e sei mesi di residenza nell'arcipelago.

Codesta forma di Governo, promossa da' coloni bianchi, non rispondeva in nessuna guisa alle condizioni generali del Paese. Complicata e costosa, lasciava nelle mani di una piccola minoranza ogni influenza politica, concedendo agl'indigeni, che costituivano il 99 per cento della popolazione, una larva di potere. Dalla sua applicazione nacque pertanto, in breve, un tale scompiglio, da costringere il Re ed il suo Governo a riaprire con l'Inghilterra trattative, per l'annessione delle Figi all'impero britannico.

Il Commodor Goodenough - lo stesso che gl'iśolani di Santa Cruz dell'arcipelago di Salomone uccisero tre anni or sono - con il Console signor Layard, sullo scorcio del 1873, ricevettero istruzioni di riesaminare la vecchia questione della cessione figiana. I loro rapporti fecero decidere il Governo inglese ad accettare l'offertogli dominio incondizionatamente. Ma gl' indigeni non erano contenti di questo, e le antiche resistenze de' Capi ricomparvero. Ad appianarle arrivò a Levuka, nel 1874, Sir Hercules Robinson, Governatore di Melbourne, uomo politico di molto tatto ed acume.

Il sentimento della propria debolezza dinanzi alle agitazioni dei

1 Aveva facoltà di decidere in ultimo appello. 


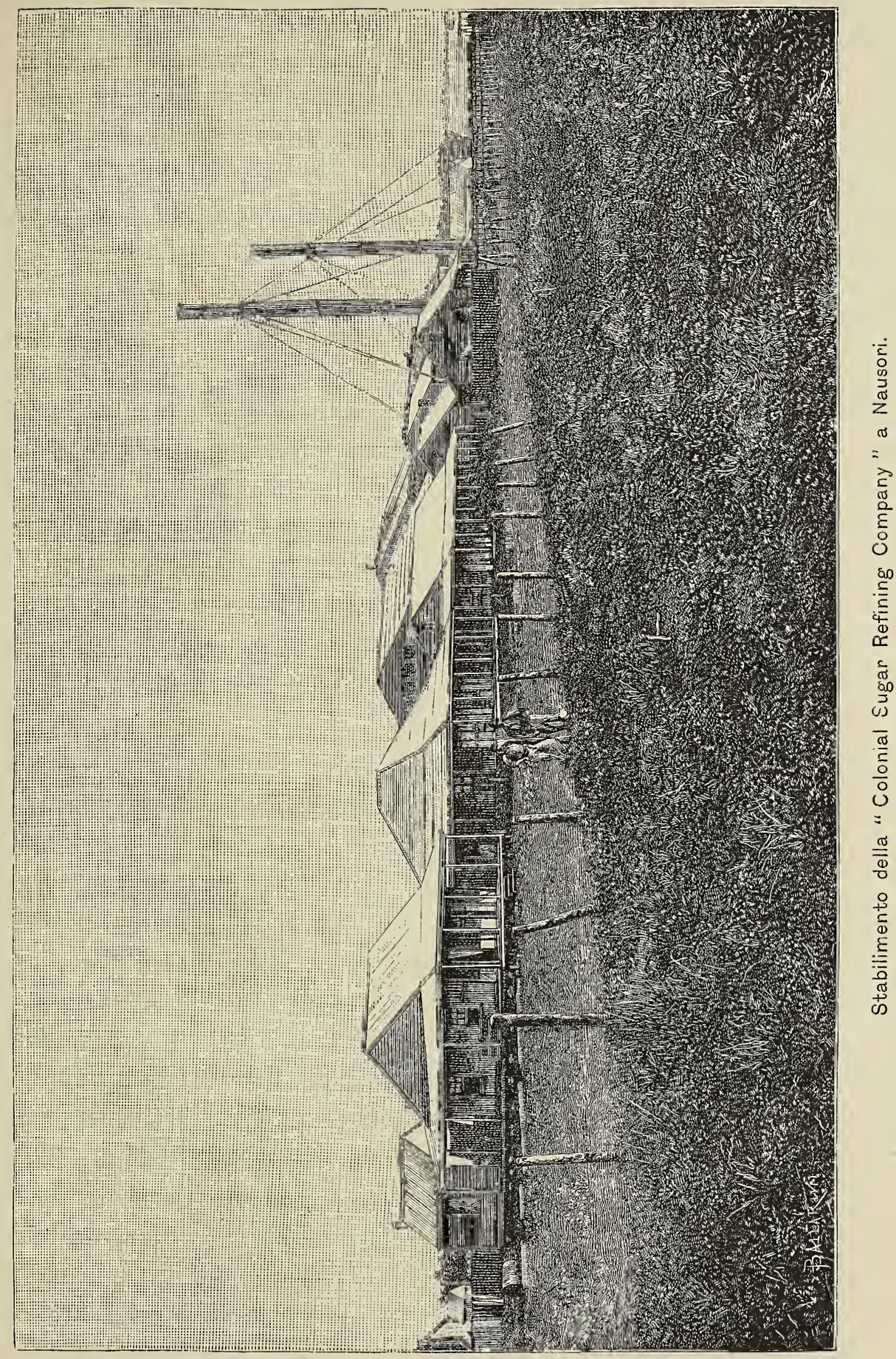



bianchi, decisi ad imporsi, aveva già fatto breccia nell'animo degli indigeni: Re e Capi erano ormai convinti che l'opporsi ancora alla volontà di gente così potente e tenace non avrebbe che peggiorata la loro sorte e tolta ogni tranquillità al Paese; perció, rassegnati, subirono il giogo, e profittando della missione di sir Hercules Robinson, trasferirono tutti i loro poteri alla Corona d' Inghilterra, che nominò al Governo dell'arcipelago sir Arthur Gordon.

Il $1^{\circ}$ settembre 1875 la nuova Amministrazione coloniale entrava in pieno esercizio.

La lunga serie di peripezie, che caratterizzano l'andamento dell'annessione figiana, peripezie da noi rapidamente delineate, mette in viva luce quanta circospezione ed avvedutezza i governanti inglesi sanno spiegare nel fondare colonie.

Naufragano sulle coste figiane, popolate da selvaggi, navi inglesi, i cui equipaggi vengono massacrati, ed il Governo invia i suoi marini militari a riconoscerle, a farne l'idrografia, per evitare altri disastri. Missionari, artigiani, commercianti inglesi prendono stanza nell'arcipelago, attrattivi dalle sue ricchezze, ma vorrebbero un appoggio immediato contro la barbarie degli abitanti, e l'Inghilterra manda sui luoghi funzionari intelligenti e coscienziosi ad investigare, studiare e riferire.

Dal 1856 al 1875, cioè durante 19 anni, il Governo, costantemente alle vedette, segue il corso degli avvenimenti, rifuggendo da ogni intempestiva ingerenza, e determina d'intervenire solo quando egli è ben certo che la sua azione diretta, reclamata da ragguardevoli interessi inglesi, avrà efficacia; però, nel frattempo l'opinione pubblica in Inghilterra ha potuto rendersi conto dei vantaggi della creazione di una colonia in quelle regioni, e servire di faro al Governo.

Ammirabile procedimento! Non è un Ammiraglio che, in nome della dignità nazionale offesa da orde selvaggie, pianta alle Figi la bandiera del suo Paese, protetta da' cannoni della sua squadra, in attesa della sanzione sovrana, che la vanità de' sud- 
diti vorrà che si accordi; la bandiera inglese, nell'arcipelago figiano, la pianta il senno di un popolo, che, guidato raramente dal sentimento, sempre dalla ragione, intende aprire un nuovo campo al suo spirito d'intrapresa ed alla sua tradizionale attività.

Aggiungiamo che nel marzo del 1881 l'isola Rotumah, situata a 400 miglia da Levuka, al N. N. O., fuori quindi dell'arcipelago, fu formalmente incorporata alla colonia figiana. La sua larghezza è di circa 5 miglia, e la sua lunghezza di 16. S'innalza dal mare verso il centro, formandovi una piccola catena di monti alti 214 metri.

La popolazione indigena, divisa in due grandi famiglie, ascende a 2150 anime.

Un residente Commissario, delegato dalla capitale delle Figi, regge l'amministrazione dell'isola.

II.

\section{Governo coloniale inglese moderno - Ordinamenti governativi per gli europei e gl' indigeni.}

Perno del sistema coloniale inglese moderno è il self-Government, ossia l'Amministrazione autonoma.

La metropoli abbandona ogni colonia a' propri bisogni ed alle proprie risorse, affinchè con criteri propri armonizzi gli uni e le altre, esigendo solamente ch'essa si presti e cooperi allo svolgimento degl'interessi politici ed economici dell'Inghilterra. A garanzia della precisa osservanza di tali principî fondamentali, la metropoli delega, con il titolo di Vice Re o di Governatore, a norma dell'entità della colonia, un rappresentante della Corona, che invigila e regola l'Amministrazione coloniale, della quale egli è mallevadore presso il Governo centrale. 
Il self-Government coloniale si foggia, possibilmente, sul Governo della madrepatria, rispetto a' coloni bianchi, includendo per gl'indigeni modalità svariate, che mirano a rispettare i loro usi e costumi, dentro opportuni confini, ed a mantenerli nel loro naturale cerchio di evoluzione.

In alcune colonie, come ad esempio in quelle australiane, ove è scomparsa la gente di colore, la forma del Governo è perfettamente costituzionale, rassomigliando in presso che tutti i suoi particolari a quella dell' Inghilterra. Nelle isole Figi, il piccolo numero di europei e l'importanza numerica e storica degl' indigeni, richiesero invece un ordinamento governativo piu ristrettivo per quelli, ed uno specialissimo per questi.

Egli è dell'ordinamento relativo a' bianchi che incomincieremo ad intrattenere il lettore.

Un Governatore - Comandante in capo ed assistente dell'Alto Commissario per il Pacifico Occidentale ${ }^{1}$ - ed un Consiglio esecutivo amministrano la colonia.

Questo Consiglio si compone:

Del Capo della Giustizia, del Segretario coloniale, dell'Attorney generale - Procuratore della Corona -, del Ricevitore generale e del Commissario per le terre.

Ha per uffici dipendenti: i Dipartimenti della Segreteria coloniale, della Ricevitoria generale, delle Terre e dell' Immigrazione, e della Giustizia.

Le leggi sono elaborate da un Consiglio legislativo, presieduto dal Governatore e composto dei membri del Consiglio esecutivo - official members -, e da un certo numero di privati unofficial members - nominati dal Governo, con sanzione sovrana; in tutto, tredici membri. Per avere effetto, debbono essere state previamente discusse ed approvate dall'ora detto Consiglio, ma

1 Sir Arthur Gordon, nello assumere il Governo della Nuova Zelanda dopo quello delle Figi, conservò il titolo di Alto Commissario e di Console generale per il Pacifico occidentale. 
il Governatore può, sentito il Consiglio esecutivo, emanare disposizioni transitorie o regolamentari, sempre che tale procedura sia stata autorizzata da atti legislativi.

Le ordinanze del Governo hanno forza legale con la data della loro promulgazione, riservato essendo alla Corona il diritto di respingerle o di chiedere emendamenti, che vengono sottoposti alle decisioni del Consiglio legislativo. Una Corte suprema giudica degli affari civili e criminali, e l'appello alla Camera di Consiglio - Privy Council - è concesso per somme, in disputa, del valore di lire sterline 500 e al disopra.

Nelle cause in cui bianchi e indigeni sono promiscuamente implicati, i Giudici sono assistiti da speciali Assessori. In ogni aitra causa criminale giudicano le Assisie.

Il Corpo della Magistratura, inclusovi il Capo, Presidente, annovera 13 membri.

Fra le leggi organiche di maggior importanza che il Governo coloniale ha promulgate voglionsi citare: quella dei Titles to Land - Titoli per le terre - e l'altra dell' Impiego manuale della gente di colore, nelle imprese dirette da'bianchi - Labors Law.

La prima, il cui scopo fu di equamente definire il diritto di possesso degli acquirenti di beni immobili, si era affacciata alla mente del legislatore gravida di ostacoli, attesa la difficoltá di risalire alla sorgente del possesso, spessissimo fondato sopra semplici affermazioni, a loro volta combattute dal diniego del venditore. Lo stato di guerra, perenne in passato, faceva sì che terre occupate in un dato momento da una tribì cadessero poco dopo in potere di un'altra, sicchè sovente accadeva che la stessa proprietá era stata donata o venduta a diversi bianchi da' Capi di diverse tribù.

Il Governo coloniale dovè accertarsi della legalitá de' titoli di possesso, e ricevere infiniti reclami, respingendone molti, rettificandone alcuni, sanzionandone pochi. Ma il periodo delle controversie è chiuso, ed i provvedimenti legislativi presi resero ormai intangibili e negoziabili i titoli europei di possesso nell'arcipelago. 
Attualmente il Governo vende, a Suva, terreni al prezzo di lire sterline 300 per un'acre, una metá pagabile subito, l'altra a tre mesi scadenza; cede terreni a doppio prezzo di quello di vendita, in esperimento, per due anni, se il valore de' terreni ceduti è inferiore alle lire sterline 300 , e per cinque, se è superiore, restituendo i $2 / 3$ della somma pagata quando non sia riuscito l'esperimento. Le perizie degli agrimensori della Corona, in caso di contestazione, sono regolate da arbitri.

La legge sull'impiego manuàle della gente di colore - figiani, polinesiani e indiani - ebbe per oggetto di allettare anzitutto gl'indigeni al lavoro, con un onesto compenso, e contemporaneamente di offrire braccia per l'agricoltura e le industrie al bianco, che altrimenti non avrebbe potuto ottenerle, il clima non consentendo immigrazioni europee. Essa però, nell'opinione de' bianchi, presenta lacune ed esorbitanze: vincola troppo la loro azione, arresta l'emulazione fra le varie tribù, ed obbliga a soverchie formalità. Il Governo, per contro, la reputa indispensabile, per frenare gli abusi derivanti da smodati desiderî di guadagno, a danno degl'indigeni, 'per abituare questi a considerare il loro benessere inseparabile da quello de' nuovi padroni. Comunque, se eccessi esistono nella sua applicazione, il senso pratico sassone non tarderà ad eliminarli.

In forza di detta legge qualsiasi contratto relativo al servizio personale degli indigeni, per più di un mese, dev'essere fatto in presenza del Magistrato indigeno distrettuale, stipendiato dal Governo, e indicare la ferma, il salario, la quantitá e qualità del vitto, del vestiario, il genere di alloggio, i mezzi di trasporto sul luogo del lavoro e quelli di rimpatrio.

Se gl'indigeni si allontanano dalla propria provincia o isola, allora l'appaltatore ha l'obbligo di chiedere l'autorizzazione dell'Agente generale dell'immigrazione, che nel caso di assenso, fa egli stesso formolare il contratto dal Magistrato stipendiato.

Sono esclusi dal lavoro i ragazzi al disotto di 14 anni, non accompagnati da' parenti o da appositi sorveglianti. 
Il costo annuo di un lavorante figiano varia dalle lire sterline 8 alle 16.

I lavoratori polinesiani - provenienti, in maggioranza, dalle Nuove Ebridi - si trovano sotto l'immediata tutela del Governo, che li recluta, li introduce nel Paese, per distribuirli ai richiedenti, per una ferma di tre anni, dietro congruo versamento di danaro, e li rimpatria. Malgrado ciò il loro numero si assottiglia ogni anno, preferendo essi recarsi nel Queensland (Australia) o nella Nuova Caledonia. Sono eccellenti lavoranti, inferiori forse ai figiani per intelligenza, ma molto piu docili, costanti ed affezionati al padrone. Costano annualmente da lire sterline 11 a lire sterline 17, 10. I figiani ed i polinesiani sono retribuiti con manufatti di loro scelta.

Provvedimenti poco dissimili sono presi circa gl'immigranti indiani, il divario consistendo: nella durata della ferma, che è di cinque anni; nell'obbligo di permanere dieci anni nella colonia, per avere il diritto al rimpatrio a spese del Governo; e nel pagamento mensile, in argento, dopo il primo anno di soggiorno. Il loro costo annuo varia dalle lire sterline 13,14 scellini a lire sterline 10, 11 scellini e 6 pence.

Codesta organizzazione del lavoro della gente di colore, con il rapido incremento della colonia europea, in una contrada eminentemente agricola, non risponde già più alle richieste di braccia dei possessori delle grandi piantagioni ed officine; e recentemente questi hanno dovuto dornandare al Governo di poter introdurre, per proprio conto, immigranti cinesi.

Passiamo all'ordinamento politico indigeno.

Il territorio figiano si divide in tredici provincie e tre distretti chiamati yasanas; e le provincie si suddividono in centotrentanove distretti, chiamati takina.

I distretti comprendono varie città o villaggi, ossia una o più qali - tribù -, e le città una o più mataqali - famiglia.

Delle dette provincie quattro oltrepassano i diecimila abitanti, sette i cinque, e due il migliaio. 
Gli yasanas contano meno di duemila anime.

La provincia è governata da un Roko-Tui - Gran Capo -, il distretto da un Buli - Capo -, la città o villaggio da un Matanivanua - persona influente -. Queste cariche, che sono ereditarie, rimangono sempre subordinate al beneplacito del Governatore. I Buli dei distretti della montagna - Colo - sono esclusivamente scelti dal Governo.

Dipendono dai Roko-Tui, oltre i cento trentanove Buli, dodici Magistrati indigeni, tredici Commessi provinciali, e molti Commessi assistenti, funzionari tutti, che ricevono emolumenti dal Governo coloniale.

Ogni provincia ha un Constabile di polizia, pagato dalla Direzione di polizia e da essa dipendente.

I Capi della cittá ricevono dalla popolazione il trattamento dovuto alla loro posizione, in conformità agli usi indigeni.

I Buli dispongono di scritturali per lo stato civile e di sorveglianti per gli orti e giardini, pagati sugli introiti di apposita tassa provinciale.

Il buon ordine è mantenuto nelle città e villaggi dai poliziotti indigeni, che sono pagati sui proventi della citata tassa.

Villaggi, distretti e provincie hanno un Consiglio, che tratta ed assetta gli affari locali nella rispettiva giurisdizione.

I Consigli dei villaggi, composti dal Capo della città e dagli anziani dei mataqali, si riuniscono mensilmente, o più sovente se è necessario, e discutono intorno alle piantagioni, alla costruzione delle case, alla nettezza della cittá, alla costruzione e manutenzione delle strade, in una parola, intorno a tutte quelle materie, che dovrà poi esaminare il Consiglio provinciale. Risolvono le piccole vertenze interne.

I Consigli provinciali si compongono dei Buli, dei Matanivanua, dei magistrati indigeni stipendiati e degli indigeni più influenti della provincia, sotto la presidenza del Roko-Tui. La convocazione del Consiglio è semestrale, cioè ha luogo nei mesi di gennaio o febbraio, e di giugno o luglio. In questi Consigli ogni 
Buli è tenuto a riferire dettagliatamente sull'andamento del proprio distretto e sulle migliorie da introdursi nei vari rami dell'amministrazione distrettuale. I Roko-'Tui, presidenti, riassumono le discussioni, e sottopongono le deliberazioni prese, con circonstanziato rapporto, al Governatore, per l'ulteriore sanzione.

Vi ha finalmente un gran Consiglio annuale di tutti i RokoTui, di due Buli, per provincia, eletti dal Consiglio provinciale, dei Magistrati indigeni stipendiati e dei Commessi provinciali. Questo gran Consiglio, convocato nel mese di novembre, nella città disegnata dal Governatore, discute intorno ai bisogni delle varie provincie sulla base dei rapporti annuali, che i Roko-Tui debbono presentare all'uopo. Le discussioni vanno, sotto forma di risoluzioni, sottomesse all'approvazione del Governatore, alla chiusura del Consiglio, e successivamente all'approvazione del Comitato dei regolamenti figiani, composto di bianchi e d'indigeni, e del Consiglio legislativo, che le trasforma in leggi. Apre e chiude il gran Consiglio il Governatore in persona.

La giustizia è amministrata da una Corte distrettuale e da una Corte provinciale; l'una, presieduta dal Magistrato indigeno stipendiato con l'assistenza, se occorre, del Buli, siede ogni due mesi, e giudica delle infrazioni di carattere correzionale; l'altra, composta di un europeo almeno, e di un Magistrato indigeno stipendiato, siede ogni tre mesi e giudica dei reati passivi di lire sterline 20 di multa o di tre anni di carcere. La Corte provinciale è Corte di appello, in materia correzionale.

Esiste un bilancio pubblico indigeno delle entrate e delle uscite, che il Governo coloniale controlla, assumendosi l' incarico della vendita degli introiti, in natura, e versando nelle Casse provinciali il denaro superante le spese. Nel 1883 il bilancio figiano presentó un civanzo di piu che duemila lire sterline.

Il Governo inglese intese adottare alcunchè di simile all'originaria organizzazione politica e sociale del Paese interessando direttamente gl'indigeni nel maneggio della cosa pubblica, e facendo progredire questa invigilandone esso stesso il corso. Vero si è 
che in parecchi casi i funzionari figiani errano, nell'esercizio delle loro funzioni, ma poichè si tratta di una razza intelligente, gli errori medesimi, messi in evidenza dal Governo coloniale, diventano infine ammonimenti salutari. D'altronde, sarebbe stato difficile, se non impossibile, il sostituire funzionari bianchi agl'indigeni, e sempre sarebbe stato costosissimo; inoltre, i Capi figiani, oggi coadiuvatori leali del Governo coloniale, si sarebbero opposti a tale sostituzione, gelosissimi come sono delle loro tradizionali prerogative e della loro influenza tra i conterranei, e sarebbero divenuti diffidenti, sospettosi e nemici delle autorità europee.

Il popolo, per servirci di una sua espressione, si sente: avere voce nelle sue terre, chè sebbene rimanga ancora molto da fare a suo pro, non si può negare, che l' indirizzo prescelto dal Governo non sia dei più corretti.

Meglio di qualsivoglia ragionamento varranno a dimostrare le aspirazioni di quel popolo i brani seguenti, eloquentissimi nella loro semplicità, di una lettera diretta nel 1878 dal gran Consiglio dei Capi alla Regina d'Inghilterra:

« Abbiamo goduto pace e contentezza nell'anno or ora spirato. Nessun male o molestia ha contristato le nostre provincie.

"Un affare per noi urgente e che ci tiene in ansie è l'educazione dei nostri figli. Desideriamo che s' istruiscano in ciò che puó giovar loro individualmente, e rendere benedetta la nostra terra.

« Noi apprezziamo il lavoro, perchè sappiamo che l'ozio è colpa. L'impiego del tempo è ottima cosa, per ogni individuo, dacchè gli procura i mezzi di sovvenire ai suoi bisogni, ma desideriamo che la nostra gente lavori, per conto proprio, nelle sue proprie terre; ch'essa pianti molto, costruisca buone case, per provvedere alle necessità della famiglia, che i suoi villaggi sieno tenuti puliti, le sue case ben riparate. Noi chiamiamo tutto ció la pace e la prosperità. 
«Il nostro popolo, per vie diverse, si trova spinto e indotto a lavorare lontano dal suo tetto, lasciando mogli, figli, parenti ed ogni cosa, in casa, nella più amara e miserabile condizione. Quindi egli cade in povertá e desolazione.

«V. M. ignora probabilmente i nostri usi e costumi. Vogliamo richiamare l'attenzione di V. M. sui compensi offerti ai nostri lavoranti dagli europei. Il pagamento ricevuto, in moneta o natura, è presto dissipato, mentre se i lavoranti stessi rimanessero a casa, o vicino, invece di andare lontani, il guadagno non sarebbe mai tutto perduto, e vi sarebbe sempre benefizio.

« Siamo molto riconoscenti a V. M. per quella parte del messaggio che c'informa come V. M. non ha intenzione di sottoporci alle leggi britanniche, nei loro particolari. Questa notizia ha dato pace al nostro spirito, e l'ha pur dato l'annunzio che V. M. vuole conservarci i costumi e le tradizioni delia nostra terra, fin dove non sono nocivi, e permetterci quelle leggi, che meglio possono convenirci ed essere da noi comprese ».

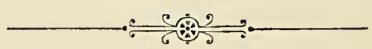




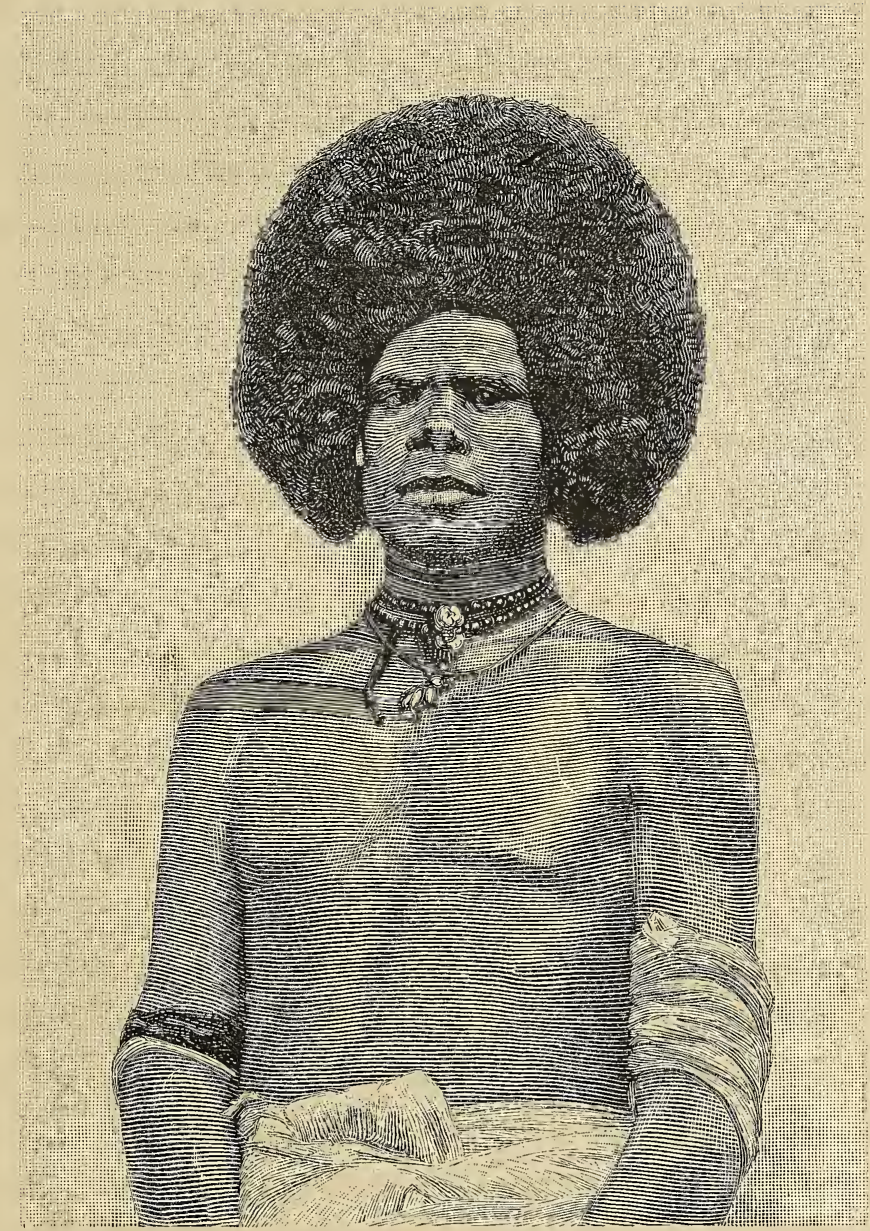

Montanaro figiano. 



\title{
CAPITOLO IV.
}

\author{
C I N N I ET TOIOGIOI
}

I.

Popolazione indigena - La rosolia - Educazione e religioni moderne.

VarI scrittori riferendosi a quei passi delle leggendarie tradizioni del popolo, che possono avere fondamento di verità, affermarono che due secoli fa la popolazione dell'arcipelago ascendeva a trecentomila abitanti.

Nel 1840 il Commodoro Wilkes degli Stati Uniti, in occasione del suo viaggio di esplorazione oceanica, la valutó invece a 133,500 anime.

Cosi rimarchevole diminuzione sarebbe comunemente attribuita alle guerre intestine incessanti ed a morbi importati da navi straniere. Due delle più gravi epidemie, la lila - deperimento - e la dissenteria sono ricordate nei canti indigeni con patetiche frasi :

"Porte chiuse e silenzio nei vuoti villaggi ed estinte tribú, in cui nessuno è rimasto per coltivare la terra, costrurre il forte, portare la clava ».

Possono forse sorgere dubbi od incertezze sulla smisurata diminuzione di abitanti rispetto al passato remoto, ma non ne 
esistono affatto riguardo agli ultimi tempi, in cui l'accordo è unanime, tra i bianchi residenti e gl' indigeni, nel sostenere che, in questa seconda metà del secolo, il decrescimento della popolazione è stato marcatissimo, raggiungendo la sua massima intensità durante la rosolia, che infierì nel 1875.

Le circostanze che originarono il terribile flagello e lo accompagnarono, meritano qui di essere mentovate, per la loro eccezionalità e gli effetti disastrosi che produssero.

Re Thakombau e due dei suoi figli, Timoteo e Giuseppe, ritornavano a Levuka, da Sydney, il 12 gennaio 1875, sulla nave da guerra inglese Dido. I Principi, che avevano lasciato l'Australia, affetti da rosolia, parevano perfettamente guariti all'arrivo, per cui le autoritá sanitarie di Levuka ristettero dal prendere a loro riguardo la benchè menoma precauzione; e parecchi europei e moltissimi indigeni poterono salire a bordo, per complimentare i reali passeggieri. Il germe del morbo non tardò a propagarsi in tutto l'arcipelago, dopo lo sbarco a terra di quella gente, chè quando i primi sintomi apparvero era troppo tardi per impedirne lo sviluppo. I Principi ricaduti ammalati, si trovavano continuamente attorniati da una moltitudine di persone, le quali in nessuna guisa volevano separarsi da loro, e subivano naturalmente gl'influssi deleterî del malore. Gl' indigeni si rifiutavano di seguire le prescrizioni mediche dettate a salvaguardia della loro salute o per la loro guarigione; ammalati, uscivano di casa e andavano a bagnarsi in riva al mare o ai fiumi, ove la dissenteria e le congestioni acceleravano la loro fine.

In talune provincie, uomini e donne, vecchi e giovani, tutti, senza distinzione, erano mietuti dalla morte, e le canoe erano insufficienti per il trasporto delle salme sulle opposte isole, ed il tamburo funebre, ${ }^{1}$ non cessava mai dal far udire i suoi lugubri suoni. Perirono approssimativamente quarantamila figiani!!

L'annessione dell'arcipelago all' Inghilterra datava da poco,

1 Il tamburo sostituisce fra i figiani la campana. 
quindi l'immaginazione popolare volle rintracciare in essa la cagione dei mali, che affliggevano il Paese. I bianchi furono creduti, moderni untori, gli autori del flagello, credenza che agli occhi figiani acquistava conferma dal fatto, che più di rado cadevano ammalati, e quasi sempre guarivano. Il malcontento si estese presto, trovando riscontro fra le tribù pagane, non domate, dell'interno di Viti-Levu, che lo colsero a pretesto delle loro aggressioni, sulla costa, contro pacifici ed inermi indigeni cristiani.

Scesero i pagani, nell'aprile 1876, alla marina, piombando improvvisamente su quattro o cinque villaggi indifesi, ai confini delle provincie di Nadi e Nadroga, sui villaggi situati in riva al fiume Siga Toka, e sul villaggio Jatabiu, per metterli tutti a ferro e fuoco.

Codesti attacchi non erano che i precursori di altri piu violenti, giacchè prima dello spirare del mese un formidabile nerbo di pagani montanari trovavasi giá pronto sulle alture dell' isola, per nuove scorrerie.

Sir Arthur Gordon, il Governatore, determinò intanto di recarsi in persona nella città di Nasaucolio, quartiere generale degl' insorti, a fine di conferire con i Capi, persuaso di potere indurli a miti consigli.

L'esito della conferenza non corrispose alle sue aspettative, e le truppe indigene fedeli, che lo avevano preceduto, dovettero, sotto il comando del suo aiutante di campo Capitano Knollys, dare l'assalto a Nasaucoko.

I montanari ebbero la peggio, rimanendo per sempre sconfitti.

È da quel tempo che il Governo mantiene sui monti una stazione militare permanente, che ha per base il forte Carnavon, e che fa amministrare i distretti delle montagne da Commissari governativi.

Nel corso degli enunciati eventi la violenza del morbo si era sensibilmente attutita, ma la popolazione continuó a decrescere per altri tre anni, cioè sino a tutto il 1878 .

Le statistiche governative incominciano a segnalare un leggero aumento di popolazione nel 1879, dando sopra 108,924 abi- 
tanti, il 279 per mille di aumento. L'ultimo censimento, che è del 1881, fa ascendere la popolazione figiana a 115,635 abitanti, dei quali 61,836 maschi e 53,799 femmine.

Tornando un istante alla leggenda figiana, dobbiamo dire che essa ripetutamente accenna a terre popolate un giorno, e poi deserte, e ripopolate in appresso, grazie alla forza di vitalitá della razza. Le condizioni dei tempi sono peró mutate, e perchè il figiano possa, nel suo nuovo ambito, ricuperare l'antica energica espansione, fa mestieri ch'egli ricorra a nuovi espedienti, segnatamente all'abbandono dei suoi villaggi e delle sue città, malsani per la vicinanza di paludi. Non sará cosa piana il convincerlo di questa e di altre imperiose necessità igieniche, conservatore tenace quale si è sempre mostrato dei costumi ed usi degli antenati. Ogni più stringente argomento si spezza oggi ancora dinanzi alla sua imperturbabile logica.

«Perchè cambiare? egli domanda. Non sono forse nato qui? Non è qui forse che i miei padri nacquero? Non hanno essi forse vissuto qui e non sono essi qui morti?

« Essi bevevano della stessa acqua che noi beviamo, passeggiavano negli stessi viali, abitavano le stesse case, mangiavano dei medesimi frutti. Perchè non potremo noi vivere e morire a modo nostro? »

L'educazione correggerà i pregiudizi popolari, e le future generazioni vorranno, probabilmente, comprendere ció che la presente si rifiuta d'imparare.

Questo popolo è infatti capace di coltura e pertanto di progresso: i due terzi della popolazione sanno già leggere, ed un quinto sa scrivere. Nelle scuole elementari in cui s'insegna a leggere, scrivere, conteggiare, ecc., si ottennero risultati non al disotto di quelli, che si ottengono nei paesi piu inciviliti.

L'istruzione impartita sui primordi dai missionari wesleyani venne poi maestrevolmente condotta da insegnanti indigeni, la qual cosa prova come il figiano non soltanto sia atto ad imparare ma altresì ad insegnare. 
L'educazione morale ed intellettuale presso i popoli nell' infanzia, com'è il figiano, impartita dai bianchi, non puó andare disgiunta dall'educazione religiosa cristiana, ed il vanto di essere stati i primi ad ammannirla, nell'arcipelago, spetta ai missionari wesleyani, che provenienti dall'arcipelago di Tonga, vi si stabilirono nel 1835, in momenti critici e pericolosi.

Le isole subirono, a grado a grado, l'influenza cristiana, e talvolta come per incanto, sul litorale; ma nelle montagne di Viti-Levu il paganesimo resistè lungamente.

Però continuarono a sussistere varie superstizioni e varie costumanze barbare, di cui citiamo le più strane:

Gli ululati notturni del cane sono creduti annunzio precursore di morte; e cosi pure il gironzare del gatto fra i piedi di un uomo; il raspare dei topi, ne' pressi della tomba di una donna, dinota che la poveretta non fu abbastanza casta in tempo di sua vita.

L'indigeno senza prole è oggetto di pietà e di disprezzo fra le tribù, ed è completamente perduto agli occhi de' suoi, il giorno in cui la moglie lo abbandona per darsi ad un altro. Egli si dispera e si reputa al livello dell'animale più immondo; la sua casa è negletta, nessuno lo aiuta più a preparare le sue vivande, a coltivare il suo giardino; gli anziani e le tribi del vicinato cessano di fargli visita e di consultarlo: egli non è più che un miserabile, un guca.

Così è inibito ai parenti prossimi di parlare fra di loro e di mangiare alla stessa mensa; e quest'ultima è inibizione che colpisce anche i coniugi. I mariti si allontanano sovente dalla moglie, ritenendosi cosa sconveniente, per un uomo ammogliato, il pernottare regolarmente nella propria casa.

Le donne, segnatamente quelle delle montagne, si tatuano, estendendo il tatuaggio, oltre a quelle parti che il likic copre, anche alle mani, per eccitare l'ammirazione de' Capi, allorquando preparano i cibi ad essi destinati. Quelle di media età o vecchie tratteggiano di azzurro gli angoli della bocca, per indicare, secondo 
alcuni, che sono madri, e secondo altri, per nascondere le traccie lasciate dagli anni. ${ }^{1}$

Oggi l'intero gruppo delle Figi è nominalmente cristiano; quasi ogni villaggio ha il suo ministro indigeno, che è pure maestro di scuola, possiede la sua chiesa, la sua scuola, entrambe erette, spesso spontaneamente, dalla popolazione e sempre mantenute da essa.

Anche i cattolici fondarono una missione nelle Figi a Levuka, nel 1844, missione dipendente dalla Società di Maria che ha sede a Lione in Francia. Conta dodici sacerdoti europei e sei indigeni, ed educa un migliaio di bambini.

Il Governo coloniale decretava nel 1880 l'impianto di due scuole professionali per gl'indigeni.

\section{II.}

\section{Cerimonie funebri del passato e del presente.}

Se v'ha metro che possa dare, con evidenza, i mutamenti avvenuti nelle credenze e nei costumi di un popolo, è certamente quello che emerge dalla differenza esistente fra due forme consecutive ed immediate di rito, relative ad un medesimo culto.

${ }^{1}$ L'operazione del tatuaggio richiede parecchi mesi prima di essere compiuta: è assai dolorosa ed arreca infinita noia.

I figiani narrano che gl'indigeni dell'arcipelago di Tonga inviarono alle Figi uno de'loro, per prendere notizie intorno alluso del tatuaggio. Costui, nel fare ritorno, ripeteva, lungo la via, per non dimenticarla, la formula: Tatuate le donne, non tatuate gli uomini, ma, sul punto di riunirsi ai suoi conterranei, preso da forte distrazione, inverti quella formula, e indusse quindi gli uomini delle Tonga a tatuarsi invece delle donne. Per tal modo verrebbe spiegata la differenza, che si riscontra attualmente, nell'uso di tatuarsi, fra i due arcipelaghi. 
Nascite, matrimonî e morti sono i maggiori avvenimenti dell'esistenza, su cui il pensiero ed il sentimento umano si posa piu insistentemente, per domandare manifestazioni ed atti pubblici, che esprimano la potenza di questo e l'intensità di quello. Le cerimonie concernenti tali avvenimenti si debbono quindi reputare come l'espressione della coscienza storica di un popolo, ed è mestieri invocarle ogniqualvolta si voglia farsi un concetto esatto del momento di evoluzione di una data razza. Ció detto, per il lettore, che ami sapere quale trasformazione si sia operata nella vita figiana, in un lasso di tempo relativamente breve trentaquattr' anni - riportiamo dall'opera interessantissima del reverendo William, Figi and Figians, la descrizione dei funerali di un Re, che visse e morì pagano, e quella di un gran Capo, che negli ultimi tempi si era convertito al cristianesimo:

«Era la mattina del 24 agosto 1845 quando mi si annunziò che il Re era morto, e che si facevano preparativi per sotterrarlo; ma ebbi a dubitare della cosa. La spaventevole parola preparativi mi spinse ad affrettarmi; peró non potei arrivare in tempo alla casa del $\mathrm{Re}$, ed a riguardo della sorte di due donne era giá troppo tardi. L'effetto della scena era opprimente. Una masnada di assassini pronti al delitto mi circondava; tuttavia non esisteva confusione alcuna, nè si menava rumore; regnava invece orrida e penosa calma. La natura sembrava essere venuta in aiuto, per dare maggior risalto alla tetra scena; non il menomo soffio di vento; la luce penetrante nel vestibolo mortuario permetteva di distinguere ogni oggetto con insolita chiarezza. Si sarebbe detto che tutto fosse pietrificato, ed uno strano sentimento mi invase, al punto di credere ch'io stesso mi fossi trasformato in una statua. Non potevo parlare: agivo incorisciamente, e mio malgrado mi accovacciai al suolo nella positura di coloro che non erano determinati ad assassinare. Il mio arrivo ebbe luogo in un momento di tregua apparente, durante cioè la crisi della morte; ed a quello strano silenzio debbo attribuire la mia emozione, giacchè ero ormai famigliarizzato con assassinî di simil genere. 
«Due gruppi di gente occupavano, sedute al suolo, il centro di una grande stanza; ed in mezzo a ciascun gruppo vi era una persona sostenuta da varie altre, coperta da un largo velo. A lato di ciascuna persona velata stavano otto o dieci uomini robusti, i quali tenevano in mano l'estremità di una corda passata due volte attorno al collo di quegl'infelici, e si disponevano a strangolarli. Ritornato in me stesso, vidi il gruppo piu lontano muoversi, gli uomini allentare la corda, e le donne togliere il largo velo e farne un cuscino per la vittima. Tolto il velo, alcuni fra coloro che avevano cooperato a consumare il delitto, sorrisero di compiacenza contemplando il viso contraffatto di una madre, come se il suo cadavere fosse un oggetto decorativo. Gli sforzi convulsivi della povera creatura che si trovava vicino a me dinotavano ch'essa viveva ancora. Era una donna robusta, e qualche esecutore giocosamente invitava chi le stava vicino ad avere pietá di lei ed a porgerle aiuto. Finalmente le donne dissero: « $\mathrm{E}$ morta ». Cadde la corda fatale; e, quando il velo fu alzato, riconobbi morta l'ubbidiente moglie e l'instancabile domestica del vecchio Re!

«Lasciai le donne aggiustare i capelli della vittima, ungere d'olio il suo corpo, dipingere il suo viso di rosso, e adornarla di fiori, e mi recai a vedere le spoglie del fu Tui-Cakau. Con mia sorpresa estrema lo trovai in vita. Era ammalato, ma completamente in sè, e preso dalla tosse, portava la mano al petto. Intanto la moglie Regina ed un domestico erano intenti a cuoprirlo di un fitto strato di polvere nera, ed a legargli alle braccia ed alle gambe un certo numero di nastri bianchi, fatti a rosette e pendenti a lunghe strisce. La sua testa era avviluppata in un turbante rosso, assicurato da un filare di piccole e bianche conchiglie. Al collo portava una collana di avorio. Per completare il suo costume, secondo l'usanza figiana, egli aveva un lungo e nuovo masi, il cui strascico si perdeva in numerose pieghe ai suoi piedi. Nessuno sembrava compreso di dolore, e si sarebbe detto che l'intera tragedia fosse soltanto una crudele parodia.... 


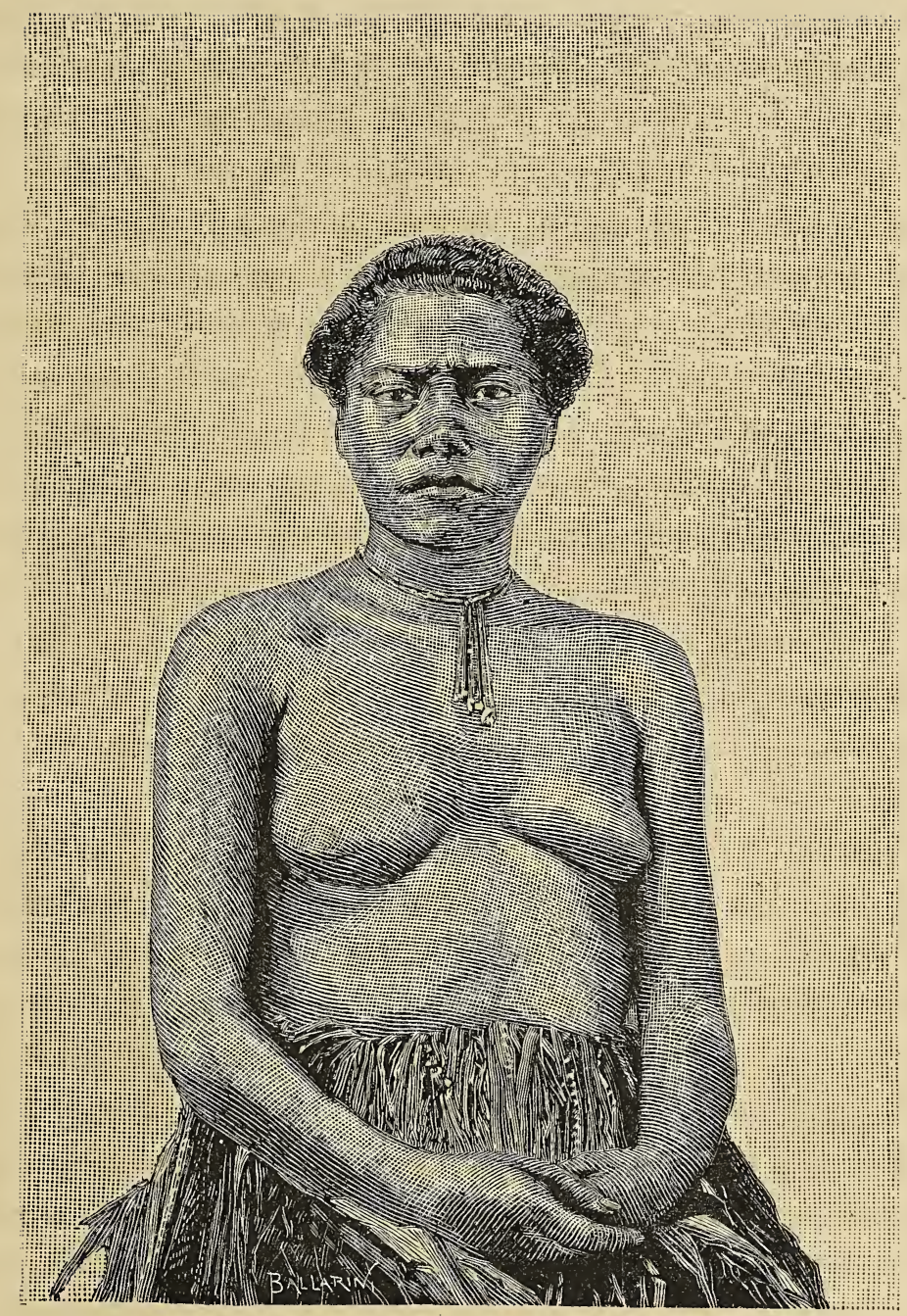

Donna figiana. 

«Ero venuto per chiedere al giovine Re la vita delle donne, ma mi parve poi dover mio il chiedergli quella di suo padre. Però, riuscendo in ciò, potevo essere causa di altri assassinî in avvenire. Con la mente perplessa, il sangue agitato ed i capelli ritti, mi avvicinai al giovine $\mathrm{Re}$, che non potevo guardare senza inorridire. Egli mosse graziosamente incontro a me, mi abbracciò e prima ancora che avessi aperto bocca, mi disse: "Guardate! il nostro padre è morto ». - « Morto! esclamai in tuono di sorpresa; morto no!» - «Si, rispose egli, il suo spirito è partito. Voi vedete il corpo muoversi; ma fa ció senza coscienza di sè ». Sapendo che sarebbe stato inutile discutere su questo soggetto, gli dissi che il motivo principale della mia visita si era di pregarlo insieme coi miei colleghi «di amarci e d'impedire che altre donne fossero state strangolate $r$. Replicò: «Là ve ne sono solo due, ma debbono bastare. Senza la presenza di voi missionari, noi la finiremmo con tutte le donne che voi vedete sedute in giro». La Regina, che si fingeva addolorata, esclamò: «Perchè non mi strangolate?» ed il Re le rispose che fra gli astanti nessuno era tanto elevato in grado da poterla soffocare.

«Fu dato ordine di ritirare i cadaveri, e noi ci allontanammo. Incontrammo una leggiadra femmina, unta d'olio e vestita con un nuovo likic, che portava una lunga canna, all'estremità della quale vi era un recipiente contenente un litro circa d'acqua, con cui, a misura che i cadaveri erano portati via, annaffiava la soglia. I corpi delle donne furono deposti a' due fianchi di una canoa, e quello del vecchio Re, a poppa, sotto una zanzariera. Vicino al Re prese posto la Regina, munita di un ventaglio per scacciare gl' insetti; tolsero al vecchio i suoi ornamenti e lo ricuoprirono di stoffe e di stuoie, ed ammucchiarono terra sulla sua persona. Dopo che una considerevole quantitá di terra era stata gettata sul suo corpo, lo si udi tossire.

«Una famiglia residente sull' opposta riva, Vanua-Levu, godeva del privilegio di dare un uomo vigoroso per essere sotterrato con il Re, a fine di recarsi prima di lui presso il cerbero 
figiano ed impedire a costui di nuocere al Re. Nel caso, di cui io fui testimonio, non fu possibile procurarsi l'uomo adatto, ed il vecchio Capo dovè affrontare il guardiano delle tenebre privo della difesa di un compagno.

«Il giorno seguente incominciò il Kana-bogi, ossia il digiuno fino alla sera. Questo digiuno è osservato durante dieci o venti giorni. Alcuni, in segno di lutto, si rasero la testa soltanto, altri si tagliarono la barba. Le donne si scottarono il corpo, e l'ordine fu dato che cento delle loro dita fossero tagliate, ma solamente sessanta furono amputate. Una donna morì in seguito a questa amputazione. Ogni dito fu messo fra le due parti di una canna spaccata, e portato nella casa del Re. Non si è mai usato strappare denti. Alcuni esprimono il loro dolore radendosi a corona la testa.

«Durante i giorni di lutto per un Capo, gli adulti sparano armi, danzano ed in generale fanno chiasso. Giovani bendati tentano di portare a nuoto una corda sopra una nave; quelli che vi riescono sono reputati futuri guerrieri di grido. Le donne della plebe non possono mangiare nè carne nè pesci, ed alle mogli dei Capi è fatto divieto, per tre mesi, di toccare con le mani il proprio cibo. Il mare che bagna la costiera, per un'estensione al largo di quattro miglia, è dichiarato tapì, ossia non si puó pescare dentro tale zona; e le noci di cocco in un raggio di sei miglia dentro terra, a partire dalla costa, sono pure sacre.

"Dopo i funerali, e ad un intervallo di tempo stabilito, vengono osservati i seguenti usi: nel quarto giorno si fa il vakaviduilo - lamento amaro -, che consiste nel fare l'uno all'altro il racconto della corruzione a cui va soggetto il corpo del defunto; il quinto giorno ha luogo il vakadrede - cagione di allegria -, che consiste in giuochi buffoneschi, ai quali prendono parte gli amici del defunto, con lo scopo di dimenticare il loro dolore; verso il decimo giorno le donne si muniscono di corde, di granate, di scudisci, e con questi oggetti battono liberamente chicchessia, ad eccezione degli alti Capi; gli uomini debbono cercare allora di fuggire il piu presto possibile, ma non mai reagire. 
«I funerali sono festeggiati anche dagli amici lontani, purchè la notizia del decesso giunga loro entro l'anno.

«Ogni piroga che arriva sui luoghi, per la prima volta, dopo la morte di un gran Capo, deve portare all'alberatura il loloku - insegna di lutto -, che consiste nel fissare un lungo masi all'albero maggiore o all'antenna, ovvero nel legare, in alto, una funicella munita all'estremità di un dente di balena, che tocchi l'acqua. Prima di ancorare, la vela e il loloku debbono essere tuffati in mare.

« La cerimonia finale, in onore del defunto Capo, è il lawa-nimate, cioè l'esecuzione di qualche lavoro eccezionalmente grande od importante, come la costruzione di una piroga, la tessitura di una balla di stoffa o di un rotolo di stuoie, una pallottola di treccia, il tutto in omaggio alla memoria del Capo, il cui nome è dato al lavoro ultimato.

«In alcuni siti un lungo seguito di donne cammina in processione portando una cesta verde colma di sabbia bianca per spargerla sulla tomba; una parte della comitiva canta in tuono lamentevole: E-ui-e, mentre l'altra risponde: E-yara; l'effetto di questa cantilena è solenne ed aggradevole. Nel caso che il Capo si sia annegato in mare, ovvero sia stato ucciso e mangiato dai nemici, il Coloka - cerimoniale - è strettamente usato come se il Capo medesimo fosse morto di morte naturale. Codesto fu il caso quando Ra-Bithi, l' orgoglio di Somo-Somo, perì in mare; settanta delle sue mogli furono sacrificate. Cosi pure dopo la notizia del massacro del popolo di Namena nel 1839, ottanta donne furono strangolate per tenere compagnia agli spiriti de' loro mariti assassinati ».

Vediamo ora il mutamento di costumi avvenuto in poco piu di un quarto di secolo. È sempre il reverendo William che narra:

"Il giorno 19 aprile del 1879, Tui Thakow, uno de' più potenti Capi del gruppo, morì. Al ritorno di una sua visita ad un'isola vicina, egli aveva accusato un certo malessere, e messosi a letto nella sua grande abitazione, non ne uscì più vivo.... 
«... Quando un gran Capo muore è necessario che il Re sia presente a' suoi funerali. In questa occasione, a fine di prender parte alla mesta cerimonia, re Thakombau e due dei suoi figli s'imbarcarono sopra una nave da guerra, alla volta di Taviuni dov'era il defunto. Partiti da Levuka nel mattino si giunse la sera a Taviuni, che è situato a breve distanza, nell'interno e di fronte al villaggio o città di Somo-Somo. Era notte al nostro arrivo colá, e la prima cosa che udimmo fu una successione di singolari suoni provenienti da terra, che riconobbi essere opera delle mogli del fu Capo, che soffiavano dentro corni di conchiglia. Codeste manifestazioni di rimpianto non erano del gusto del principe Giuseppe, secondogenito del re Thakombau, educato a Sydney.

«Il domani mi recai a perlustrare la cittá, e la prima casa in cui m'imbattei fu quella del defunto Capo. Era un gran fienile con quattro porte molto bene ideate. I pilastri di sostegno erano tronchi d'alberi di cocco e l'ossatura bambù; stuoie rivestivano la parte inferiore delle pareti; una lunga pezza di tappa, stoffa indigena, divideva il fabbricato in due parti, in una delle quali trovavasi il Capo deposto nella sua cassa ed avvolto in una quantitá innumerevole di stuoie; nell'altra, sua moglie (la figlia del re Thakombau) era seduta in terra facendosi fresco con un ventaglio.

«Bellissima figiana ella era, chè, quantunque avesse molto pianto, pure non cessava di essere veramente avvenente. Intesi dire che pareva molto piu bella prima di tagliarsi i capelli. ${ }^{1} \mathrm{Li}$ aveva tagliati in segno di lutto. Tutti, maschi e femmine, in ogni villaggio, avuto notizia della morte di Thakow, si erano tagliati i capelli per la stessa ragione. Dopo essermi allontanato dalla casa del Capo, mi misi a passeggiare nel villaggio, ove si facevano i

${ }^{1}$ Ai tempi del cannibalismo le zitelle soltanto portavano i capelli lunghi ed allo stato naturale, adornandoli con fiori ne' giorni di festa; per le maritate sarebbe stato futile ed imprudente il conservare una lunga capigliatura, e però esse portavano i capelli corti. 
preparativi per la festa del dimani. Gruppi d'indigeni grattugiavano mandorle di cocco e canne di zucchero, uccidevano maiali, tagliuzzavano tartarughe ed apparecchiavano ignami, tarò, banane, ecc. Altri indigeni portavano alla casa del defunto vivande, che l'isola di Taviuni e le isole vicine mandavano in dono alli vedova; si fermavano dinanzi alla casa, si sedevano sul suolo, formando circolo, e dopo aver delegato uno de' loro per parlamentare con la vedova, facevano consegna dell'offerta e si ritiravano. Il delegato, nell'atto in cui la vedova lo ringraziava, applaudiva con battimani.

"Oltrepassata la casa del Re e di suo figlio, casa isolata da tutte le altre, vidi pile enormi d'ignami ed un numero sterminato di tartarughe. A mezzogiorno i marinai ed i soldati della nave da guerra britannica scesero a terra nel sito ove erano seduti tutti i Capi di tribú, venuti per associarsi al funerale, e si schierarono lungo la via che dalla casa del defunto conduceva alla fossa. La processione incominciò allora a muoversi lentamente. Il prete cattolico, accompagnato da' suoi accoliti, con ceri accesi, aprì la marcia; poi comparve la cassa mortuaria portata da dieci vigorosi indigeni; indi la vedova, sola; il rappresentante di S. M. Britannica, il Luogotenente Governatore delle Figi; seguivano infine il Re Thakombau con la sua Corte, e tutti i Capi per ordine di rango. Giunta la processione presso la fossa, che era stata fatta a breve distanza dall'abitazione del defunto, fu letto il servizio divino, e la cassa, fasciata di stuoie, si fece scendere nella fossa. I militari sbarcati fecero in quel punto tre scariche, in bianco, di moschetteria, e la processione mosse verso la casa del defunto seguita dalla truppa di sbarco, che fece ritorno al proprio bordo. Dopo ciò incominciarono i tocchi funebri con i corni di conchiglia, e gl' indigeni si adunarono per bere il kava....» 
III.

\section{Industrie indigene.}

I figiani sono essenzialmente agricoltori, quantunque alcune tribù abbiano nome di pescatori, falegnami e costruttori. Tutti indistintamente lavorano la terra, ed è rarissimo il caso di trovare maschi, dall'età tenera alla più avanzata, che non possiedano il loro campicello e non lo coltivino da sè; in certi luoghi anche le donne hanno il proprio giardino, a cui attendono personalmente.

Le derrate coltivate sopra maggiore scala sono: gl'ignami, il dalò (Calucasia esculenta), il masi, con la cui corteccia fanno stoffe, il kawai, la banana, il tabacco e la yaqona o yanqona o kava.

Il dalo, base del loro nutrimento, si coltiva in due maniere distinte: all'asciutto, ovvero nell'acqua. In questo secondo caso il dalò richiede un vasto sistema d'irrigazione regolato e mantenuto da tutte le mataqali aventi diritto di possesso sui terreni della piantagione.

Fra le piante esotiche, che, introdotte nel Paese da pochi anni, il figiano coltiva, ma con minor passione delle precedenti, figurano il kumala, la cassava, il granturco, goli aranci, il caffè. La coltivazione della canna da zucchero diventata assai retributiva, dopo che funzionano stabilimenti per la sua fabbricazione sul fiume Rewa, non ha mancato di richiamare l'attenzione degl' indigeni, ed alcuni villaggi vi si dedicano già per conto proprio.

I figiani seguono, nel coltivare la terra, le antichissime consuetudini paterne, le quali, difettose sotto il rapporto economico, costringono a molta accuratezza, e danno raccolti di buona qualità. Gli utensili agrari sono semplicissimi : un'ascia ed un coltello 
per sgombrare la terra, e il dokó (bastoncino puntuto) per rimuoverla. Gli europei dicono che il dokò è preferibile all'aratro.

Ogni indigeno è tenuto a coltivare il proprio campo, ciò che non lo esime dal lavoro collettivo, in primavera, che è la stagione delle seminagioni e piantagioni.

In quell'epoca, tutto un villaggio o cittá lavora la terra di un solo individuo, incominciando da una mataqali e passando successivamente per tutte quelle che comprendono il villaggio o la città. Questa mutua assistenza si estende talune volte ad altri lavori agricoli, che non sieno la seminagione o la piantagione, esigendosi dal proprietario della terra un'abbondante distribuzione giornaliera di alimenti e nulla piit.

Capi di tribù e di famiglia hanno sempre insistito sulla bontà e convenienza del lavoro collettivo, che, a parere loro, mantiene, da un lato, vivi gli usi industriali del popolo, procaccia comodità ed agiatezza alle masse, e, dall'altro, mette alla portata di ogni indigeno, qualunque sia la sua posizione sociale, il cibo necessario ed un buon alloggio.

La manifattura del masi - stoffa indigena per abiti -, delle stuoie, delle stoviglie e di altri articoli domestici, è particolarmente affidata alle donne, rappresentando in certi distretti una vera specialití locale. Ogni anno si fabbricano migliaia e migliaia di stuoie, e quantità enormi di masi e di altri generi difficili a classificarsi per numero e qualitá. Queste industrie, un tempo rilevantissime, declinarono con la frequenza dei contatti con gli europei, ma accennano attualmente a risorgere alquanto. ${ }^{1}$

${ }^{1}$ Il processo in uso presso i figiani, nella manifattura del masi o malo, presenta speciale interesse, tanto più che in alcune parti dell'Oceania, nella Nuova Zelanda, ad esempio, ove in altri tempi tale manifattura fioriva, attualmente è del tutto perduta. Eccone la succinta descrizione:

Si stacca, dapprima, la corteccia dell'albero del malo (Broussonetia), a strisce della maggiore lunghezza possibile, e poi queste si mettono nell'acqua per facilitare la separazione dell'epidermide, mercè una larga spatola di conchiglia. L'epidermide rimane immersa nel liquido alcun tempo, indi vien 
Le donne di Lakemba sono insuperabili nella lavorazione del masi; quelle di Malaku fanno zanzariere della stessa stoffa assai ricercate. Kandau produce solidi masi, detti liti, che, eccezionalmente vengono fabbricati dagli uomini.

Il likir dalle donne si fa con la corteccia del vau (specie d'hibiscus) o con filamenta di radiche selvatiche, od anche con alcune erbe.

Per ordine d'importanza, la manifattura delle stuoie vien dopo quella del masi. Esistono infinite varietá di stuoie, ed un indigeno intelligente puó, a primo colpo d'occhio, riconoscerne la provenienza, ogni isola mostrando certe particolaritá nel materiale adoperato o nel modo di prepararlo. Si lavorano stuoie rozze e se ne

battuta sopra una specie di ceppo levigato e piano, con una piccola mazza di legno. Volendo dare maggior consistenza al masi, si battono insieme due striscie di detta epidermide sovrapposte, che il glutine contenuto nelle fibre rende aderenti. Battuto il masi, in tal guisa, si ottengono pezzetti di stoffa, e questi, uniti fra di loro con amido di taró o con arrowroot bollito, danno nastri di parecchi metri di lunghezza; varî nastri congiunti longitudinalmente, seguendo lo stesso metodo di unione, determinano stoffe di 12 a 25 metri quadrati di superficie, su cui le donne possono, con disegni a colori, esercitare a piacimento tutta la loro abilità artistica. Questo genere di lavoro, che merita menzione, è eseguito nel seguente modo: Su di una tavola convessa e lunga alcuni metri, si collocano parallelamente e distanti l'una dall'altra 3 centimetri circa, liste sottili di bambù, della larghezza di 1 centimetro; e, negli interstizi si depongono alcuni pezzi ricurvi, formati da costole di foglie dell'albero del cocco. Su quest'ordegno si stende la stoffa, e confricandola con il colore che dà il lauci (Aleurites triloba), essa riceve naturalmente l'impronta del colore medesimo nelle varie parti in cui subisce la pressione sostenuta dalle liste, e dai pezzi ricurvi sottostanti. Quando si debba dipingere il tappeto con quadrelli, con grandi tondi o con linee punteggiate ed irradiantisi dal centro, allora fa mestieri che il colore sia più denso; e si ricorre ad una spazzola per stenderlo. Il colore, chiamato dagli indigeni kasa, se fu ben preparato, prende, una volta asciutto, un bel lucido.

I tappeti cosi dipinti servono comunemente di zanzaricra, oggetto che i figiani apprezzano moltissimo. Con i masi non raddoppiati, nella battitura, si fanno leggerissimi turbanti, che hanno tutta l'apparenza del velo. 
lavorano delle finissime per pavimento, vele, letti, culle. Le grandi misurano 8 metri sopra 5, variando la larghezza della treccia da 3 a 6 centimetri. Le stuoie per vele variano in larghezza da centimetri 50 a metri 1.30, ed in lunghezza da 100 a 300 metri; la lunghezza usuale è di 5 a 7 metri. Le peggiori treccie sono quelle di Rewa e le migliori quelle di Moala. Si adopera, per fare stuoie rozze, la foglia dell'albero del cocco, ma, per tutte le altre, si usa invece la foglia del pandano nano o del Pandanus odoratissimus, ovvero si usano giunchi côlti nelle paludi.

L' industria de' panieri è pure rimarchevole nell'arcipelago, poichè colá se ne producono dei bellissimi e svariati per disegno e forma.

Un altro ramo de' lavori d'intrecciatura, nei quali i figiani sono assai abili, è la manifattura de' ventagli. Essi riescono parimenti nell'industria delle reti. I pescatori di tartarughe fanno le proprie con le fibre della corteccia della noce-cocco o del pandano. Le maglie delle reti sono di foggia europea, ma i pesi del fondo, invece di essere di piombo, sono di conchiglia.

I figiani godono poi di una riputazione di meritata superiorità, in confronto degli altri isolani oceanici, nella fabbricazione della ceramica, che comprende numerosi oggetti: tazze, scodelle, piatti, pentole, brocche, ecc. Si servono, per cosiffatta fabbricazione, di una argilla rossa o azzurra, a cui mischiano sabbia. Ricorrono ad istrumenti molto semplici: una specie di sostegno bucato in mezzo, quattro spatole (tata) ed una pietra rotonda e levigata. I fregi li eseguiscono mentre l'oggetto è ancora umido. Dopo avere esposto, durante alcun tempo, al sole il vasellame, lo ricoprono di uno strato di leggiero combustibile: foglie, paglia, ecc., al quale appiccano il fuoco; ${ }^{1}$ quando il combustibile è consumato, passano sulla superficie esterna di ogni oggetto una certa resina di pino, ed il lavoro si considera ultimato. La fabbricazione della ceramica appartiene esclusivamente alle donne, ed in ispecie alle mogli de' pescatori.

${ }^{1}$ È questa che abbiamo chiamata cottura al sole, al capitolo II, pag. 147. 
Nell'isola Vanua-Levu si fa del buon sale, ma alquanto scuro di colore, col sistema dell'evaporazione naturale; lo si conserva in cestine presso il fuoco. Vi si affumica il pesce.

Molti indigeni si dedicano alla costruzione di canoe. Pare che un tempo fosse concesso soltanto a talune tribù di eseguire quel genere di lavoro, ma adesso il privilegio è cessato. I falegnami costituivano, non è molto, una casta col nome di falegnami del $R e$, avente un Capo proprio. ${ }^{1}$ Gl' indigeni delle isole degli Amici sono costruttori più abili e marinai più arditi de' figiani; tuttavia, gli isolani di Tonga hanno adottato le canoe di questi ultimi ed imitato la loro attrezzatura.

La lavorazione delle loro armi è pure degna di attenzione: clave o mazze e lance, d'infinite varietà con impugnature spesso artisticamente intagliate od intarsiate di avorio o madreperla; archi della İunghezza di 2 metri, ricavati da' rami pendenti della rizofora. I fabbricanti d'armi producono anche eleganti suppellettili in legno ed avorio di balena, come vassoi, piatti, cerchi, recipienti per kava, forchette da cannibali, ecc. Gli utensili adoperati, finchè rimase ignoto l'uso del ferro, consistevano in ascie di selce legate saldamente ad un manico di legno, ed in coltelli di conchiglie.

L' industria della pesca è largamente esercitata nelle Figi, e quella che concerne la tartaruga, ricercatissima, ha poi presso gli indigeni un'importanza capitale. La pesca di questo anfibio si fa generalmente sotto gli ordini di un Capo, che stabilisce la parte di guadagno spettante a ciascuno. I pescatori di tartarughe spesso ricevono, al ritorno da una felice escursione, doni di alimenti e di terreni; ma questa pesca non è priva di pericoli a cagione delle insidie de' pesci-cani e degli scogli. Talune volte si catturano le femmine mentre vanno a deporre a terra le uova.

1 Vi sono nelle Figi quattro classi di canoe: il velovelo, il camakau, il tabilai ed il drua. Ciascuna implica modificazioni nel contrappeso e si distingue nei particolari dello scafo. 
In un giorno si possono prendere da tre a quattro tartarughe; nonostante, accade sovente di non prenderne alcuna durante parecchi giorni. Sono le donne che fanno il commercio del guscio di tartaruga.

Gli indigeni, come abbiamo veduto, non disdegnano il lavoro, perchè ogni idea di abbondanza è da loro vivamente accarezzata.

Nessun figiano vorrá mai considerare la sua casa come ben governata, se in essa non vi sará abbondante provvista di suppellettili e di cibi per la famiglia, i dipendenti, i visitatori. Egli per essere soddisfatto vuole che il suo granaio sia colmo, il suo campo ferace, abbondino in casa le stuoie stese al suolo o rotolate, le masserizie, le stoviglie, ecc., per ogni contingenza, e vi sia anche il suo lenzuolo mortuario. Molto da mangiare, molto da regalare, è l'ideale sublime del figiano, abbia egli qualità di Capo o d'infimo suddito.

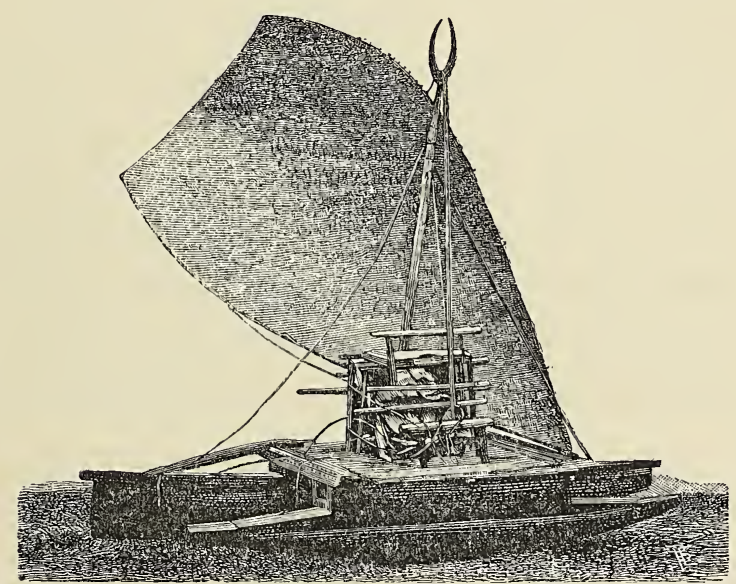

Piroga figiana. 



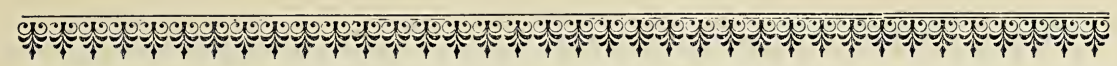

\title{
CAPITOLO $\mathrm{V}$
}

\author{
I.
}

\section{Agricoltura moderna.}

L'Arcipelago, con una grande varietà di suolo, copiosi fiumi e speciali condizioni climatologiche, è soprammodo appropriato alla coltivazione di disparatissimi prodotti; e ragguardevole, per distesa di terreni coltivabili, presenta al capitalista, semprechè non difettino le braccia, una sorgente di straordinari benefizi.

È difficile il dire con precisione a quanto ammonti la superficie agricola del paese, non essendosi peranco potuto misurarla in tutto l'interno, molto boscoso, ma si presume che l'area utile complessiva delle isole sia approssimativamente di 20 milioni di ettari.

La superficie coltivata, nel 1880, era di 12,140 ettari, ed il Governo coloniale, in quell'anno, aveva alienato, in concessioni e vendite, 11,040 ettari di terreno coltivabile. A tutto il 1880, l'insieme delle alienazioni governative calcolavasi di 56,407 ettari.

Viti-Levu ha una superficie di 4110 miglia quadrate; il suo suolo non è de' migliori per coltivare i cocchi, ma è ottimo per il riso e la canna da zucchero. 
Vanua-Levu misura in superficie miglia quadrate 2420, e possiede molte foreste ricche di alberi pregiatissimi per la qualitá del legname. È la sola isola dell'arcipelago in cui si trovi il legno sandalo; il suolo produce abbondanti cocchi.

Le isole secondarie di Ovalau, Kaudavu, Govo o Kovo Taviuni ed altre, hanno feracissimo suolo, adatto, segnatamente, alla coltivazione del cocco.

Nell'arcipelago, senza dire de' legumi, tuberi e frutti de' paesi tropicali o semi-tropicali, che vi allignano ottimamente, la terra può produrre in abbondanza riso, granturco, canna da zucchero, cotone, caffe, zenzero, china, cocchi, arrowroot.

La coltivazione del riso non è stata ancora esperimentata, ma quella del granturco e della canna da zucchero, iniziate da pochi anni, diedero eccellenti risultati.

Il cotone, ${ }^{1}$ articolo che richiamò prima d'ogni altro l'attenzione del pubblico commerciante e industriale sulle isole Figi, fu introdotto, nel 1863, dal Dr. Brown, console per gli Stati Uniti di

${ }^{1}$ Per darsi alla coltura del cotone occorre, anzitutto, preparare il terreno, e perciò abbattere gli alberi, sradicare i cespugli e lasciar quelli e questi disseccare sul suolo, appiccandovi il fuoco quando soffia vento. Indi conviene marcare il terreno in ordine di qualità. Nel terreno migliore si debbono separare gli alberi cotoniferi, l'uno dall'altro, alla distanza di 4 piedi, e disporli in filari distanti fra loro 8 piedi; dove la terra è più povera la distanza fra albero ed albero dovrà essere da 6 a 7 piedi, e quella fra filari da 3 piedi a $3 \frac{1}{2}$. Si scaveranno fossatelli di 9 pollici di larghezza con un piede di profondità, e, portando i semi in sacchi, se ne deporranno 5 o 6 in ogni fossatello; $\operatorname{così}: \circ$, circa un pollice sotto il suolo, ricuoprendoli leggermente, con la mano, di terra fino all'orlo. Con tempi umidi, dopo tre o quattro giorni, i semi incomincieranno a germogliare, e dopo altri quindici giorni sarà necessario difendere gli steli dalla violenza del vento.

Cresce il cotone rapidamente per modo che in due mesi e mezzo può raggiungere 5 piedi di altezza. Occorre però cimarlo di un pollice tosto che sia pervenuto a piedi $4 \frac{1}{2}$ di altezza; allora l'arbusto spiegherà i suoi rami. Il fiore sbuccia tre mesi dopo la seminagione; è di color giallognolo dapprima, e poi diventa rosso. Il frutto si forma e si sviluppa completamente 
America. Nel 1867, il cotone delle isole era venduto, in grana, a navi mercantili, al costo di lire italiane 040 la libbra inglese, equivalente a lire 3 08, sgranato. Le migliori località per coltivarlo rintracciansi nelle fertili pianure sabbiose, che circondano le diverse isole, e nelle quali esistono strati di argilla nera e di terra bruna. Alcuni fra i primi piantatori commisero l'errore di servirsi di terreni coperti d'erba, e le spese del dissodamento assorbirono ogni profitto.

Sono appena trascorsi sette anni dacchè il caffè venne importato da Ceylan e coltivato con metodo, sebbene se ne incontrassero prima qua e là alcuni arbusti, che si supponevano importati dal Sud-America. Oggi la coltura del caffè è assai generalizzata fra i coloni europei, e gl' indigeni, dal canto loro, sotto il savio impulso del Governo, hanno incominciato in varî distretti a seguire il buon esempio. Per questo genere di coltura i piantatori scelgono siti ben riparati dai venti alisei del S. E. evitando l'ombra degli alberi, che è dannosa.

Il caffè ${ }^{1}$ non ha bisogno nelle Figi di occupare terreni ele-

nel lasso di circa sei settimane; con il caldo, il guscio si apre presto, ed in due o tre giorni il fine cotone setoso può essere raccolto.

Per il primo anno, il rendimento sarà piuttosto scarso, ma aumenterà sensibilmente il secondo, essendo scemate di molto le spese di preparazione del suolo. L'albero del cotone può vivere tre anni, producendo nel terzo quanto aveva prodotto nel secondo. In seguito, converrà sradicare gli alberi e ricominciare la seminagione fra gl'interstizî dei filari esausti. La esperienza ha dimostrato che il cotone cresce vantaggiosamente nel medesimo suolo, senz'alcuno speciale artifizio, durante venti anni. La seminagione vuole essere fatta sul finire di novembre e in dicembre.

${ }^{1}$ La coltivazione del caffè nelle Figi varia pochissimo da quella che è in uso negli altri paesi produttori di tale coloniale, ma è necessario porre mente, nella scelta del terreno, che il caffè sia difeso dall'aliseo di Sud-Est, e non ombreggiato da alberi. Il seme, disposto con le consuete cure, germoglierà presto e potrà dare frutti sette mesi dopo la seminagione. Una piantagione su grande scala sarà sempre vantaggiosa, purchè la distanza fra i semi venga regolata a seconda della ricchezza del suolo, avvertendo che.in un buon terreno la produzione, nel terzo anno, sarebbe esuberante, ove i semi fossero disposti 
vati quanto altrove: bastano 300 metri dal livello del mare, e non 1000 come a Ceylan. Coltivato sulla costa, dà grani piccolissimi.

Lo zenzero ${ }^{1}$ è indigeno delle Figi, ove cresce rigoglioso, nei boschi, allo stato naturale; però, quello che i coloni bianchi coltivano fu importato da Ceylan. E un'industria agricola alla portata delle piccole borse.

La china, ${ }^{2}$ il cui valore commerciale è dovuto agli alcali che secerne la corteccia, rappresenta uno de' prodotti più proficui dell'agricoltura figiana, sia per le lievi cure che richiede, sia per lo spazio relativamente esiguo che la coltura stessa esige. Al contrario dello zenzero, i capitalisti soltanto possono trarne buon partito, giungendo a lunga scadenza la rimunerazione del lavoro, cioè fra i quattro e gli otto anni. Il capitalista, che investe nelle piantagioni di china (chinchona), somme non inferiori alle 2500 o 3000 lire sterline, può ottenere il 300 ed il $400 \%$ di benefizio.

Uno de' rami agricoli più rilevanti dell'arcipelago è indubitabilmente la coltivazione metodica dell'albero del cocco, ${ }^{3}$ colti-

a 6 piedi di distanza l'uno dall'altro. La cimatura dell'arbusto vorrà essere fatta con molto riguardo: 5 piedi di altezza non sono molti, in un terreno bene esposto, specie se è di natura vulcanica.

${ }^{1}$ Si piantano i piccoli bottoni 2 piedi distanti l'uno dall'altro, in un terreno ricco e sgombro da radici e da pietre. Il frutto arriva a maturità in nove mesi, e, raccolto che sia, vuol essere immerso nell'acqua bollente ed esposto al sole per disseccare. Lo zenzero in conserva - poichè esistono nelle Figi fabbriche di zucchero - potrebbe diventare un'industria importante.

${ }^{2}$ La china non richiede alcuna particolare coltivazione, ma si ritiene che le condizioni di clima e di suolo, necessarie al suo maggiore sviluppo, si trovino nelle Figi. $\dot{E}$ un prodotto che offre a'grossi capitalisti, soltanto ad essi, grandi attrattive, perchè sono rilevanti le spese d'impianto, ed è lungo il tempo che precede un qualche utile rendimento.

${ }^{3}$ Lubicazione migliore per il suo intero sviluppo è in prossimità del mare; le isole basse, ovvero la costiera delle isole grandi esposte alla brezza marina, sono i luoghi da preferirsi per coltivarlo. Scelto il terreno, che deve essere boscoso dacchè è il più ricco, bisogna bruciare compiutamente gli alberi ed i cespugli che lo ricuoprono, e fare una scelta di buone noci; queste deb- 
vazione che ha preso uno straordinario sviluppo in questi ultimi anni, ne' quali si sottoposero 2400 ettari al trattamento di quell'albero.

Le località che meglio convengono alla sua coltura si trovano in vicinanza del mare, o almeno vicino all'influenza dell'aria salina. Le piccole isole e le coste delle maggiori sono molto favorevoli alla coltura dell'albero, perchè esposte tutt'attorno, ed in permanenza, alle brezze di mare. Sulle spiagge piane di sabbia, e soprattutto se a livello delle acque, esso acquista il suo massimo vigore. Anche le terre elevate gli sono propizie, se vicine al mare e se di formazione geologica corallina o vulcanica. Fu detto che non cresce, nè dà frutti a grandi elevazioni dal mare; ciò sarà, trattandosi di grandi isole o continenti, ma nelle Figi si è verificato l'opposto, poichè nell' isola di Rambi, della circonferenza di $30 \mathrm{mi}-$ glia, ad un'altezza di 300 metri, ed a 3 miglia dal mare, il cocco prospera a meraviglia.

Chiuderemo queste brevi note sull'albero del cocco ripro-

bono essere piantate separatamente ad una distanza l'una dall'altra da 24 a 30 piedi, e così disposte ${ }_{\circ}^{\circ} \circ$, che è il modo più vantaggioso. Per ogni noce si fa un fossatello di 2 piedi di diametro e di 2 di profondità, attorno a cui si mette il terriccio estratto. Converrà piantare le noci in una giornata umida e piovosa, quando le noci stesse abbiano già un germoglio da 9 a 12 pollici di lunghezza. Per piantare la noce, la si terrà, con una mano, in mezzo del fossatello, il germoglio in alto, e, con l'altra, valendosi di un bastoncino puntuto le si farà cadere tutto attorno e al disopra, fino a cuoprirla, il terriccio raccolto sull'orlo, e lo si premerà, in seguito, fortemente con i piedi. In tal maniera la pioggia, penetrando gradatamente attraverso il suolo, mentre crescerà la pianta, farà sì che al termine di 12 o 18 mesi il fossatello si colmerà. Sarà provvido il piantare altri prodotti negli interstizî lasciati dalle giovani piante di cocco, nei primi 2 o 3 anni, finchè sono piccole.

Il cotone delle Figi è quello che più comunemente si usa con tale intento, poichè non impoverisce il suolo, e dà presto un congruo rendimento. Si possono seminare tre filari di cotone fra ogni filare di cocco, e, pertanto, provvedere con un'unica spesa ad un solo lavoro, alla coltivazione cioè simultanea di due prodotti. Il cotone compensa sempre la spesa. 
ducendo la descrizione che fa il signor H. Stonehewer Cooper, nella pregiata opera The Coral Lands of the Pacific, dello strano processo di riproduzione dell'albero medesimo:

"Lungo tempo dopo che la noce del cocco è venuta a completa maturità, una sostanza bianca, dolce di sapore, spugnosa, si forma nell' interno, ed origina il germe dal lato opposto ad uno dei fori dell' estremità puntuta del guscio, ossia dal lato stesso ove il frutto ha il gambo.

"La fibrosa spugna assorbe l'acqua del cocco, riempie la cavità e scioglie la dura mandorla, per combinarsi chimicamente con essa e determinare una pastosa sostanza cellulare, che prende il posto della mandorla e del latte. Spunta nel frattempo una gemma di color bianco, che si fa passaggio attraverso il mentovato foro, penetra nella corteccia fibrosa del cocco e s'innalza di alcuni pollici, esponendo alla luce ed all'aria le sue verdi foglie. Due consistenti filamenti bianchi, che partono dallo stesso punto del germe, sturano gli altri due buchi del guscio, forano la corteccia in direzione opposta e si affondano nel terreno. Guscio e corteccia deperiscono a poco a poco formando un leggero concime e facilitando in tal modo lo sviluppo della giovine pianta, che affonda sempre piu le sue radici, spinge in alto il suo fusto e spiega le sue palme ».

Il clima delle Figi è altresì appropriato alla coltura dell'arrowroot, tubero le cui seguenti varietà possono essere vantaggiosamente coltivate nelle Figi stesse : la Tacca pinnatifida (indigena delle isole Sud del Pacifico), la Maranta arundinacea (indigena delle isole Bermude), la canna coccinea, la Jatropha manihor (tapioca).

Queste varietà che crescono rigogliose in tutto il paese, sono abbondantissime nel gruppo di Yasawa e sulla costa Nord-Ovest di Viti-Levu. Gl'indigeni coltivavano un tempo una gran quantitá di arrowroot, che dicevasi, in commercio, preparato dai missionari delle isole Sud del Pacifico; presentemente lo usano, in molti distretti, come alimento. L' arrowroot è affatto trascurato dai coloni bianchi. 
Le foreste abbondano di alberi stupendi, che possono dare ottimo legname da costruzione di molte varietà.

Fra questi vi ha il vesi, adoperato dagli indigeni per costruire canoe, e la cui fibra compatta e resinosa rassomiglia a quella del mogano, anche per il colore. Vi ha pure il bau, rosso cupo, con grana chiusa e forte quanto quella dell' ebano, che riesce bellissimo quando è verniciato. È comunissimo, nelle Figi, il dilo o tamanu di Tahiti, che raggiunge spesso grandi dimensioni. Il nokonoko, legno di ferro (casuarina), vi si trova in non scarsa quantità; gl'indigeni lo usavano per la confezione delle clave. Vengono dopo: il cerua, o sandalo bastardo, giallo, di grana rosata e ricco di olio aromatico; il dakua, il dakua salusalu, varietà del Damaria australis, utilissimo pino quando è secco; il vaivai simile al tamarindo; il viriviri, molto leggiero; il vura, leggiero quanto il sughero, e molti altri alberi che sarebbe lungo enumerare.

II.

\section{Armenti - Pesca.}

Gli estesi campi delle isole maggiori sono adatti per ogni rispetto all'allevamento degli animali domestici, abbondandovi le acque correnti, durante tutto l'anno, ed i pascoli essendovi perennemente verdi.

L'arcipelago, privo d'animali indigeni, deve esclusivamente all'importazione quelli che oggi possiede. Si racconta che quando, nel 1851, si sbarcarono i primi cavalli, gl' indigeni rimasero attoniti e spaventati.

I coloni non rivolsero mai seriamente la loro attenzione su 
quel ramo d'industria agricola, e tuttavia gli armenti si propagarono notevolmente.

I bovini di razze incrociate riescono meglio degli altri; cavalli e muli prosperano, e finora nessuna malattia endemica od epidemica è venuta a molestarli. Sui montoni, puro sangue od incrociati, i quali attualmente sono immuni da malattie, non si potrebbe dire altrettanto, giacchè nei primi tempi della loro introduzione furono travagliati dal tetano e dalla carie ai piedi. Nel 1875 s'introdussero capre di Angora con felice successo. Maiali ve ne sono ovunque; molti, allo stato selvatico, vivono nei boschi. I gallinacei importati si moltiplicarono rapidamente.

Al dì d'oggi, nella colonia, la richiesta di buona carne di bue o di montone si equilibra con la produzione, a prezzi moderati.

Nel 1880, la provvista di bestiame (Live stock) era di 500 cavalli e muli, di 4000 bovini, di 3000 montoni, di 1500 capre di Angora, di 100,000 maiali e di 100,000 gallinacei.

Le Figi sono estremamente ricche di pesci di mare e di acqua dolce. Numerosissime le sogliole, le triglie, i pesci volanti, le razze, ecc., i gamberi, le tartarughe. Una specie di squalo di acqua dolce assai pericoloso infesta il fiume Rewa, ed i veri squali, in numero di nove varietá, pullulano sulle coste.

Le oloturie (bîches de mer dei francesi, o tripang dei chinesi), ricercatissime sui mercati della China, si pescano sulle coste nord di Viti e Vanua-Levu e nel gruppo di S. E., specialmente a Fulanga. Piccole barche, equipaggiate da chinesi, esercitano cotale pesca, che il Governo locale ha dovuto con recenti ordinanze disciplinare, per impedirne il prematuro esaurimento.

Di quattro specie, distinte per colore, sono le oloturie figiane: bigie, nere, rosse e variegate. La specie piu ricercata, e quella quindi di maggior valore, è la bigia, che si trova soltanto a levante del $180^{\circ}$ meridiano; raggiunge comunemente, al suo completo sviluppo, 50 centimetri di lunghezza ed un po' meno in circonferenza; vive sui banchi piani e sui fondi sabbiosi dei lagoni. 
L'oloturia nera si trova esclusivamente sui fondi di sabbia, scevri di scogli ed a profondità variabili fra 50 centimetri a 18 metri; alcune volte è lunga 70 centimetri e grossa quanto la gamba di un uomo.

Le oloturie rosse sono le piú piccole ed anche le meno ricercate; raramente raggiungono la lunghezza di 30 centimetri; vivono sui banchi di corallo e sui loro lembi foranei dove il mare frange costantemente.

Le oloturie variegate hanno colore olivastro macchiato qua e là ; assumono proporzioni pari a quelle delle maggiori oloturie nere.

I mari figiani danno anche ricetto alla madreperla, ma la pesca della preziosa conchiglia è in piena decadenza.

III.

\section{Industrie moderne.}

La fabbricazione dello zucchero, scarsa non ha guari e disseminata, va concentrandosi tutta nel grandioso stabilimento di Nausori, ${ }^{1}$ il quale, in breve, ne diverrà il monopolizzatore. Codesta industria, nello svolgersi in un paese nuovo per gli europei, e barbaro ancora, con l'impronta dei ritrovati meccanici più perfetti dell'epoca, esalta lo "spirito d'intrapresa dei suoi fondatori, e prende posto, fra tutte, in prima linea.

Seguono le industrie del cocco: copra ${ }^{2}$ e borraccia ${ }^{3}$ (fibre della corteccia o pericarpio), e del cotone.

1 Vedere per maggiori particolari il capitolo I, paragrafo III.

2 Vedere il capitolo V, TAHITI, paragrafo III, Industrie.

3 Borra. 
Anteriormente al 1870 il copra era quasi sconosciuto, nelle Figi, come articolo di esportazione, gli esperimenti fatti nel 1860, per prepararlo, essendosi risolti in un insuccesso.

La scelta della sede del lavoro era caduta sopra una località soggetta a continue pioggie, l'isola di Moturiki, ove la mandorla ammuffiva. Per ben dieci anni un prodotto, che attualmente rappresenta un valore di lire sterl. 64,000 annue, era stato negletto. L'unico uso che si facesse della mandorla del cocco, si riduceva all'estrazione dell'olio, valutata annualmente a lire sterline 8000 .

Il copra della migliore qualità è quello che si ottiene facendo disseccare le mandorle nel proprio guscio; la qual cosa richiede una casa o tettoia assai spaziosa. Bisogna evitare che le noci sieno aderenti al suolo, perchè altrimenti germoglierebbero; e bisogna inoltre lasciare intatta la corteccia, affinchè non penetri aria nell' interno del frutto da' fori del guscio. Se le noci furono convenientemente collocate si può essere certi che dopo tre mesi l'acqua sarà scomparsa, e la mandorla avrà presa la consistenza del cuoio, che conserverá indefinitamente senza subire più gli effetti del clima, dell'umiditá, ecc. Il migliore copra dà l'olio più chiaro e più dolce, e quello, che meno diminuisce di peso per evaporazione.

Per ottenere la borraccia, che non é altro che la corteccia del cocco, occorre eseguire un'operazione, che non danneggi la noce, e che un abile manuale esegue, servendosi di un bastoncino conficcato nel suolo ed appuntato in cima, con il quale scava il pericarpio e ne spoglia la noce. Le corteccie, in tal maniera preparate, sono messe in grandi serbatoi, in cui rimangono da 12 a 24 ore sotto l'azione del vapore; indi si passano al laminatoio, per ammorbidire la parte legnosa, e s'introducono in cilindri muniti di denti di acciaio che le tritano e le riducono a borraccia (coilfibre degli inglesi).

La società inglese di Rambi (Rambi Plantation Company), la più importante dell'arcipelago, relativamente a questa industria, ha macchine potenti e perfezionate, con le quali ottiene varie 
qualità di borraccia, ed in conseguenza borraccie per usi vari: scope, spazzole, cavi, ecc.

I mercati principali di quell'articolo sono l'Australia e la Nuova Zelanda. Settemila noci di cocco danno circa una tonnellata di fibra comune valutata, sul luogo, da lire sterline 7 a 15. La fibra per scope è valutata invece da lire sterline 15 a 30.

Il costo relativo, escluso quello del macchinario, varia tra lire sterline 5 e lire sterline 6 .

Un altro articolo; ma di secondaria importanza, che trova smercio sui mercati inglesi e di Germania è la mandorla della. noce candela (candle nut), così chiamata perchè, presso i polinesiani, infilzata in uno stecchetto di bambú, fa ufficio di candela. Questa noce, contiene molto olio, valutato in Europa da lire sterline 30 a 35 la tonnellata. Vive allo stato selvatico in tutte le isole dell'arcipelago, e non costa altra fatica, agl'indigeni, che quella di raccoglierla sul suolo.

Per la guerra di secessione d'America la coltivazione del cotone prese un incremento straordinario, ed anche oggidì i suoi prodotti sono fra i più stimati del mercato di Londra.

Giova dire che gl'interessi agricoli della colonia sono invigilati, con gelosa premura, dalla Planters' Association (Società dei piantatori), la cui sede è tuttora a Levuka. Annovera già piu che un centinaio di soci.

IV.

\section{Commercio.}

La posizione geografica dell'arcipelago delle Figi, rispetto agli altri gruppi d'isole del Pacifico, è così centrale, i porti figiani sono così sicuri e bene adatti, da lasciar travedere che, in un próssimo avvenire, esso diverrà l'emporio commerciale 
della zona racchiudente tutti quei gruppi. Sventuratamente, il Governo ha dovuto, di fronte alle rilevanti e indispensabili spese che l'impianto di una nuova colonia esige, sottoporre a regime doganale gli articoli d'importazione, con qualche danno per il commercio.

L'adozione di un sistema d'imposte dirette, ottimo, in teoria, e realizzabile in altre circostanze di tempo e di luogo, nella pratica, sarebbe stato nocivo, perchè avrebbe colpito coloni che, esordendo appena nelle imprese agricole e industriali, sentono vivo il bisogno di essere sciolti da ogni vincolo fiscale. D'altro lato, il Governo non poteva tentennare, ma doveva prontamente decidere e procacciarsi introiti, per acquistare credito ed ispirare fiducia. Adesso che le condizioni dell'erario si sono sensibilmente migliorate, e che il tesoro pubblico si ripromette laute entrate, con le tasse indigene, con quelle sui bolli, le successioni, gl'impieghi, ecc., verranno semplificati i diritti doganali, ed il Governo potrà far pesare meno la sua mano sul commercio.

Il bilancio della colonia, entrate e uscite, dal 1875 al 1880 inclusivo, fu il seguente:

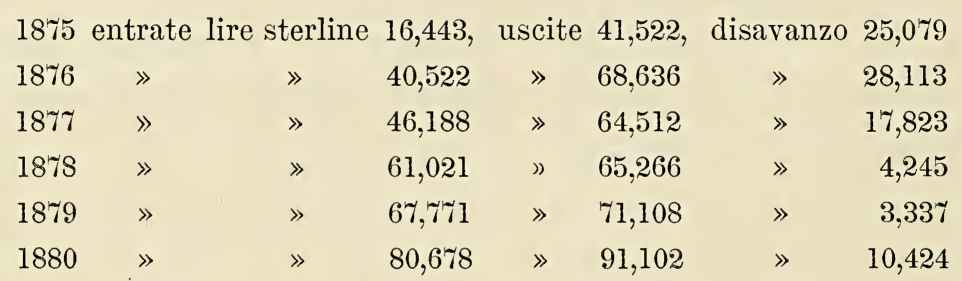

Durante undici mesi del 1881 si ebbe un civanzo, cioè lire sterline 72,486 di entrate e 72,073 di spese.

Il 30 dicembre 1880 il debito pubblico ascendeva a lire sterline 210,000 .

Le statistiche ufficiali dànno un'idea adeguata del grado di importanza commerciale che le Figi seppero già conseguire, mercè l'acume degli amministratori e la costanza degli amministrati. Esse dicono, ad esempio, che le importazioni e le esportazioni crebbero 
da lire sterline 198,264 nel 1876, a 311,252 nel 1879, e da questa cifra a quella di 493,846, nel 1882.

Le relazioni commerciali dell' arcipelago con la madrepatria e le colonie consorelle dinotano che in esso come in tutti i possedimenti britannici produzione e consumo locali rimangono sotto l'esclusiva, o quasi esclusiva, influenza inglese. Ed infatti, si ha nel 1881 un'importazione nazionale di lire sterline 240,472 contro una importazione straniera di 28,791. La quale differenza enorme dipende dai legami d'affari esistenti fra i coloni bianchi delle Figi e gli australiani inglesi, ma anche dal non essere ancora conosciute all'estero le risorse economiche figiane. Tuttavia, a Levuka alcune delle case commerciali piu intraprendenti e che lavorano su larga scala, in copra e cotone, sono tedesche; però la merce loro, imbarcata sopra navi del proprio paese, è lasciata, nella generalità dei casi, in Inghilterra.

L'importazione annovera: sacchi, calzature, biscotti, burro, cordami, tessuti, droghe, metalli lavorati, porcellane, cristalli, oreficerie, macchine, sapone, vini, liquori, thè, ecc., articoli tutti, o per la maggior parte, di consumo europeo. Gl'indigeni ricercano soprattutto i tessuti a colori gai: fasce, camicie, giacche, fazzoletti da donne, ecc.

La colonia figiana, mirabilmente situata, trova nei mercati di New South Wales, Vittoria e Nuova Zelanda un esito immediato al suo granturco, alle sue frutta fresche, al suo zucchero, alle sue borracce di cocco, mentre gli articoli di maggiore esportazione, il copra ed il cotone, seguono la via d'Inghilterra, con semplice transito in Australia.

Il commercio del copra, che fa capo a Levuka, è quasi esclusivamente nelle mani delle ditie Bush e C., Hernay e C., Hoeron e C., le quali incettano il cocco ne' dintorni, lo raccolgono in ampi magazzini, lo preparano e lo esportano col mezzo di navi a vela.

Questo commercio decadde nel 1879, per cause accidentali, e risorse nel 1880, decadendo ancora nel 1882. Una prova evidente 
della facilità con cui il commercio figiano puó trovare opportuni sbocchi, a brevi distanze, la si ha nell'incremento dell'esportazione dei frutti freschi, sul mercato di Sydney, dopo che fu stabilito fra questa cittá e Levuka un servizio mensile di trasporti a vapore. I frutti freschi esportati a Sydney: banane, ananas, granadillas (frutto della passione), rappresentavano: nel 1876 un valore di lire sterline 10, nel 1877 di 180, nel 1878 di 1642, nel 1879 di 3146, e nel 1882 di 904412.

Coordinando ora, per quantità e valore, le esportazioni degli articoli piu cospicui, si hanno:

\begin{tabular}{|c|c|c|c|c|c|c|}
\hline Per il copra & tonnellate & $4,089.15$ & Lst. & $61,353.10$ & nel & 1879 \\
\hline$»$ & $\gg$ & $7,265.12$ & $\gg$ & $108,985.10$ & » & 1880 \\
\hline$\gg$ & $»$ & $7,422.12$ & $\gg$ & $85,831.0 .6$ & $»$ & 1881 \\
\hline$\gg$ & $\gg$ & $6,883.11$ & $\gg$ & $64,787.15 .9$ & $»$ & 1882 \\
\hline Per lo zucchero & $\gg$ & 785.00 & $\gg$ & $26,687.9$ & $\gg$ & 1879 \\
\hline$\gg$ & $»$ & 593.00 & $\gg$ & $23,920.14$ & $»$ & 1880 \\
\hline$\gg$ & $\gg$ & 683.19 & $\gg$ & $23,254.1$ & $»$ & 1881 \\
\hline$»$ & $\gg$ & $1,731.00$ & $\gg$ & $58,857.8$ & $\gg$ & 1882 \\
\hline Per il cotone & $\gg$ & 373.8 & $\gg$ & 44,020 & $\gg$ & 1879 \\
\hline$\gg$ & $\gg$ & 386.4 & $\gg$ & 45,530 & $»$ & 1880 \\
\hline$\gg$ & $\gg$ & 297.14 & $\gg$ & 35,100 & $\gg$ & 1881 \\
\hline$»$ & $\gg$ & 270.15 & $\gg$ & 31,920 & $»$ & 1882 \\
\hline Per le banane & $\gg$ & - & $»$ & $5,189.19$ & $\gg$ & 1880 \\
\hline$\gg$ & $\gg$ & - & $\gg$ & $5,229.3$ & $\gg$ & 1881 \\
\hline$»$ & $»$ & - & $\gg$ & $9,044.12$ & » & 1882 \\
\hline Per la melassa & galloni & 26,534 & $\gg$ & 2,050 & $\gg$ & 1879 \\
\hline$\gg$ & $\gg$ & 19,944 & $\gg$ & 1,691 & $\gg$ & 1880 \\
\hline$\gg$ & $\gg$ & 11,286 & $\gg$ & $1,682.8$ & $\gg$ & 1881 \\
\hline$\gg$ & $\gg$ & 36,218 & $\gg$ & $5,432.14$ & $\gg$ & 1882 \\
\hline Per il granturco & staia & 71,008 & $\gg$ & $10,651.4$ & $»$ & 1879 \\
\hline$»$ & $\gg$ & $64,0 \% 2$ & $\gg$ & $9,610.16$ & $\gg$ & 1880 \\
\hline$\gg$ & $\gg$ & 34,248 & $»$ & $5,137.4$ & $\gg$ & 1881 \\
\hline$\gg$ & $\gg$ & 19,320 & $\gg$ & 3,864 & $\gg$ & 1882 \\
\hline
\end{tabular}

Il valore complessivo delle esportazioni ascendente nel 1876 a lire sterline 103,459 , raggiunse nel 1882 la somma di 190,517 . 
Gli scambi commerciali fra le Figi, l'Australia e la Nuova Zelanda si effettuano mensilmente, a mezzo di tre Societả di navigazione a vapore, di cui una è sussidiata dal Governo. Questa, che fa altresi il servizio postale, dai porti figiani abilitati alle Tonga, impiega due vapori, per mantenere facili e non interrotte le comunicazioni fra quei porti medesimi, e le isole dell' arcipelago.

I vapori mensili di Sydney sono in coincidenza con gli arrivi e partenze via Suez; quelli che muovono da Aukland (Nuova Zelanda) si estendono sino a San Francisco di California. Buoni velieri di altura trasportano in Europa parte de' prodotti indigeni e numerose flotte di velieri di cabotaggio e di barche provvedono alle necessitá del commercio marittimo locale.

Durante l'anno 1879 entrarono nei tre porti abilitati: Suva, Levuka, Loma-Loma, 131 navi di 28,967 tonnellate, e ne uscirono 125 di tonnellate 28,085. Nel 1882 si verificó un aumento nel movimento marittimo commerciale di 30 navi, corrispondente a 15,230 tonnellate.

É caso curioso, che dal 1878 in poi il movimento delle navi a vapore sia andato sempre aumentando, nella colonia, in misura uguale o quasi al movimento delle navi a vela. Infatti nel 1878 detto movimento era rappresentato da 28 piroscafi di 9557 tonnellate, e da 100 velieri di 13,623 tonnellate; nel 1881 da 45 piroscafi di tonnellate 16,356 e da 119 velieri di 19,186 tonnellate.

In sussidio al commercio, il Governo coloniale ha istituito un Ufficio di marina (Marine board), ed una Camera di commercio, composta de' piu influenti ed autorevoli mercanti ed uomini di affari della colonia. A complemento, esistono finalmente due Banche, cioè la Banck of. New Zeland e la Union Banck of Australia (limited), cinque Società di assicurazioni, un'agenzia del Lloyd ed una delle ipoteche.

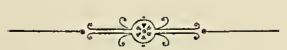





\section{CAPITOLO VI}

I.

Geologia - Orografia - Topografia.

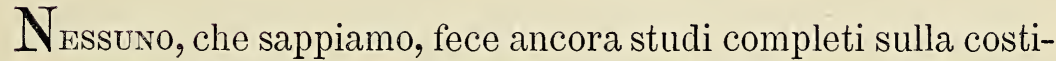
tuzione fisica dell' arcipelago figiano. Le nozioni che si hanno di geologia, orografia, topografia del paese emergono dal racconto de' vari navigatori, che in tempi diversi lo visitarono, o dalla descrizione di escursioni fattevi da viaggiatori dilettanti, senza alcun intento scientifico.

Il signor Thurston, ${ }^{1}$ attuale Segretario coloniale a Suva, rilevó nel 1865, con misure al sestante ed orizzonte artificiale, una carta dell' isola Viti; ed il signor Macdonald, in un'esplorazione lungo il fiume Rewa, studiò alquanto, in modo geologico, l' isola medesima.

L'autorità di queste due distinte persone sará quella che invocheremo nelle seguenti linee.

${ }_{1}$ Dobbiamo agli studi dell'onorevole Thurston ed ai documenti ufficiali da lui gentilmente fornitici, molti degli elementi con cui abbiamo compilato il nostro lavoro sulle Figi. 
Le isole maggiori, al pari di tutte le isole alte dell'Oceano Pacifico, sono di formazione vulcanica e coronate dalla consueta scogliera madreporica, che le ha dotate di numerosi e sicuri rifugi per le navi.

La superficie del loro suolo ondulato offre detriti vulcanici, e fra le varie rocce: basalti, trachiti, brecce e conglomerati; offre altresì terreni alluvionali o sedimentari racchiudenti fossili, animali e vegetali. Fu riconosciuta in alcune località la presenza del rame e della piombaggine.

I monti si elevano più o meno ripidamente dalla costa sino a 1200 e 1400 metri.

Codesti sono i contorni generali; quanto alla struttura intima, per farne cenno, dobbiamo riferirci ad una piccola zona dell'isola Viti, unica di tutto l' arcipelago, che sia stata esplorata.

Dal punto in cui l'Waimanu, tributario del Rewa, cessa di essere navigabile, recandosi sulle alte terre piu prossime alla città di Koroi, si domina il paese circostante, imponente e sval'iato di aspetto. Queste alte terre si compongono di rocce sedimentarie, in cui abbondano le foraminifere, ed in ogni dove s'incontrano impressioni fossili o meglio avanzi di animali e vegetali induriti da uno strato superficiale di ossido di ferro. Tali organismi sono stati completamente metamorfosati, i loro elementi cioè vennero sostituiti da quelli che costituiscono le rocce attigue. In molti luoghi si osservano grandi masse di brecce, simili a quelle dell'isola Ovalao, sparse fra le rocce stratificate nella più strana maniera. La regione intera presenta sommo interesse, potendosi notare gli strati di un antico letto marino, elevato ora circa 120 metri sul livello del mare, letto che confina con montuose masse di brecce e conglomerati di frammenti di compatte lave primitive, cementati insieme da minuti detriti della stessa materia.

A Navuso, sul Rewa, trovasi alla riva una sabbia riccamente ferruginosa sorretta da uno strato alluvionale della profondità da 1 metro ad 1 metro e 50. Da Navuso, rimontando il fiume e lasciando 
a N. E. la cittá di Mbau, s'incontra il distretto di Naitasari, nel quale vi ha un sito chiamato Waini-Kumi, ove si vedono rocce di formazione sedimentaria con la superficie quasi verticale. Su di esse scorre un filetto d'acqua, che secondo la tradizione indigena avrebbe la virtu di far crescere la barba agli adulti. ${ }^{1}$ Waini-Kumi significa appunto acqua della barba.

Lasciato Naitasari, il suolo si eleva gradatamente, ed alla biforcazione del Rewa, nei due rami Muna-Ndomi e Wai-Ndina, le sponde sinistre si mostrano scoscese e formate da rocce stratificate.

Per rintracciare le sorgenti del Wai-Ndina, bisogna traversare il distretto di Soloira e trasportarsi sulle alte montagne che sono al di là della vallata di Namosi. L'Wai-Ndina si ripiega due volte al Sud nella sua corsa, ed è in questo secondo serpeggiamento che si trovano le sponde composte di uno strato di piccole pietre rotonde, e selci, che, frammischiate con arene e sabbie, seguirono le vicende di terreni alluvionali recenti. Nel distretto di Soloira, in prossimità della città capoluogo Wani-Mbau, vi è una collina sulla cima della quale si abbraccia di un solo tratto tutta questa regione di un incantevole aspetto. L'Wai-Ndina lambe la città di Naseirau, famosa per le sue sorgenti termali, di cui alcune scaturiscono da una roccia, specie di breccia metamorfica, che presenta una bellissima superficie screziata. Da Naseirau le acque vanno a Namosi, che giace sulla riva destra, nella lussureggiante vallata Ono-Mbaleanga.

In questo punto ha termine l'accennata regione esplorata. Nel S. E., ossia al vento, le isole dell' arcipelago sono coperte da dense foreste di robusti e pregiati alberi, appartenenti a numerose varietà, fra i quali si osservano i coniferi Damara, Dacrydium e Podocarpus. Altri alberi, come il Vairai (Serianthes vitientis), il Damanu (Calophyllum Burmanni), il Vesi (Afzelia bijuga)

\footnotetext{
${ }^{1}$ Gli indigeni tengono in gran conto la barba (Vedi Darwin, Origine de l’homme).
} 
risaltano per bellezza ed utilità. Una vegetazione più leggera veste le terre basse che, apparentemente almeno, hanno dovuto essere coltivate in tempi da noi non molto lontani, quando la popolazione indigena era più fitta. Il suolo è pressochè dappertutto profondo, facile a lavorarsi, e da recenti analisi risultò ricco di humus.

Nel N. e N.O. delle isole maggiori, ossia sottovento, le terre sono comparativamente prive di foreste. I monti e le pianure hanno manti di lunghe canne ed erbe, e macchie di Casuarina e di Pandanus. Sul margine del fiume e dei torrenti, e nelle valli liparate, che raccolgono i detriti de' monti circostanti, gli alberi forestali ed i frutti comuni crescono bene.

Le Figi sono largamente provvedute di acque, frequentissime pioggie alimentando perennemente, le fonti di migliaia di piccoli torrenti, che si riversano nei fiumi. Fra quest' ultimi, per ampiezza e navigabilità, il Rewa occupa il primo posto; può essere rimontato con barche, pontoni e vapori piatti, per oltre 40 a 50 miglia dalla sua foce. Vengono dopo il Wai-Manu, il Wai-Ndina, il Wai-Mala, le cui sorgenti si rintracciano sugli alti monti dell'interno a 900 e 1200 metri dal pelo del mare. Il Sigatoka e il Ba sono corsi d'acqua, che, insieme con molti altri, vanno a gittarsi nel Thalveg di Viti-Levu.

Nell' isola Vanua-Levu i fiumi e i corsi d'acqua, in genere, sono in minor numero e meno ampî. Il Dreketi, il Lambasa, il Wai-Levu ed il Wai-Nunu figurano fra i più rimarchevoli.

Quasi ogni vallata dell'arcipelago ha i suoi ruscelli, con i quali gl' indigeni irrigano le piantagioni di dalo. 


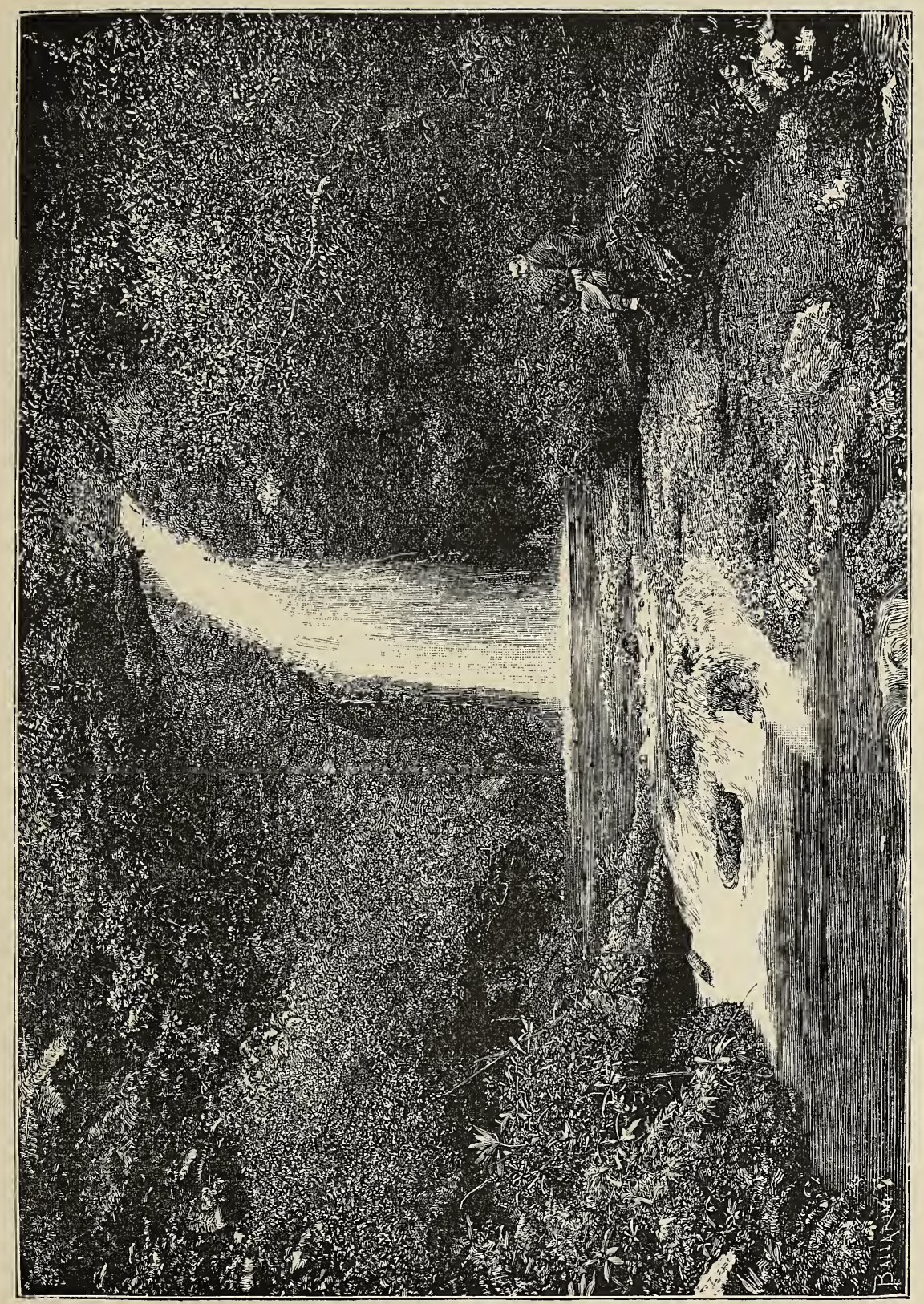



II.

\section{Clima - Neteorologia.}

Quantunque situato fra il $15^{\circ}$ ed il $20^{\circ}$ di latitudine australe, e quindi in una zona di temperature elevate, il gruppo delle isole figiane può considerarsi, riguardo a clima, confinato in una regione semi-temperata, a cagione della sua distesa e delle sue alte montagne.

La siccità regna sulle isole basse, e dal lato di sottorento delle più grandi; mentre l'umiditá, le pioggie sono proprie del lato del vento di queste ultime, stabilendo un contrasto così spiccato da determinare due climi interamente diversi.

In tesi generale si distinguono due stagioni: l'invernale o secca, da maggio a settembre; e l'estiva o delle pioggie, da ottobre all'aprile. Nella stagione estiva $i$ venti soffiano con maggiore 0 minore forza dal S. E.; nell'inverno, essi sono variabili, prevalendo alcune volte i venti del Nord, che apportano un caldo eccessivo, e promuovono ne' coloni bianchi uno stato di languore e di depressione.

Il caldo, tranne quei casi, si mantiene quasi uguale in tutto l'anno, essendo meno opprimente di quello che si esperimenta in parecchie colonie australiane.

Sulla costa, la temperatura media è di $26^{\circ} 7 \mathrm{C}^{\circ}$. Il mattino e la sera sono deliziosi. Sulle alte montagne dell' interno essa è molto piu bassa, a segno che qualche notte il freddo costringe a fare uso del fuoco.

La relativa siccità dell'atmosfera e le brezze fresche preservano il paese dalle maligne infermitá tropicali e, poichè le paludi sono ristrette per numero e superficie, non si hanno febbri miasmatiche eccessivamente deleterie. 
La stagione reputata dagli europei come la più piacevole e salubre è l'invernale.

Qui cade in acconcio il menzionare una circostanza curiosa, che prova come le stesse cause operanti nelle stessissime condizioni, sopra due razze diverse, che vivono l'una a fianco dell'altra, possono produrre effetti diametralmente opposti. L'ora detta stagione invernale, ritenuta da' bianchi più confacente alla loro salute dell'estiva, segna per gl'indigeni l'epoca della maggiore mortalità. L'estate, particolarmente nei mesi di gennaio, febbraio e marzo, è la stagione più favorevole alla salute de' figiani.

Evidentemente il caldo e l'umido conferiscono di più alla loro costituzione del freddo portato dai venti di S. E., prevalenti nell'inverno freddo, che li predispone a raffreddori ed a febbri, sovente di carattere epidemico, e fatali nelle loro conseguenze.

I mesi estivi poc'anzi citati chiamansi anche i mesi degli uragani, temuti assai dai marini e dagli agricoltori, sebbene molti ann ${ }^{\mathrm{i}}$ passino senza che le nubi mandino altra cosa che forti colpi di vento.

I più notevoli uragani di questa seconda metà di secolo furono quelli del 1866 e del 1871, che soffiarono intensamente, in tutto l' arcipelago, devastando ovunque le proprietá. Uragani di minor forza e parziali si fecero inoltre sentire fra quelle date e dopo, accompagnati da torrenziali pioggie e da un innalzamento delle acque, che causó gravissimi danni. Tuttavia, si esagrererebbe considerando l' arcipelago delle Figi come una contrada normalmente soggetta a tali pericolose meteore.

Un'idea della importanza degli uragani parziali la si puó avere nella descrizione seguente, che i signori Holmes e Shares, in alcune loro note meteorologiche, fanno delle tempeste, che visitarono il gruppo figiano nel 1879:

«L'11 e il 12 gennaio, a Mbau, si ebbero piovaschi con forti venti dal N. al N. O. e pioggia.

« L'11 febbraio, tempesta ed umiditá; dal 9 dello stesso mese al mattino del 12 non fu visibile il sole. Pioggia durante una settimana. 
«L'll dicembre, piovasco, con leggera pioggia e forte vento, che avendo incominciato dal S. E. con lampi e tuoni, girò al N. E., per indi soffiare con violenza fino a notte fatta. Il barometro corretto segnava alle 3 pomeridiane $747^{\mathrm{mm}}$. Nella notte, il vento passò all'Ovest, e divenne moderato. Il cielo era sereno il mattino del 12. Sulla costa di Mbau i danni furono lievi, sebbene quest' ultima tempesta fosse stata la più intensa dopo l'uragano del 1871.

«Il movimento orizzontale dell'aria, a Rewa, l' 11 dicembre tra le 330 e le 430 pomeridiane, fu di 40 miglia; tra le 430 e le 530 , di 50 miglia; tra le 530 e le 630 , di miglia 56.5 ; e tra le 630 e le 730 , di 56 miglia ».

Sono ancora in troppo piccolo numero le osservazioni meteorologiche, che si possiedono sulle Figi, per dedurne alcune norme, ma quelle che, illustrate da tabelle, i prelodati signori Holmes e Share insieme con il signor Nerval fecero nel 1879 in localitá diverse e di ben distinto clima: Mbau, ${ }^{1}$ Rewa, ${ }^{2}$ Tavuni, ${ }^{3}$ presentano un vero interesse speculativo e pratico.

Dai vari dati che quelle tabelle forniscono, si desume: $1^{\circ}$ che a Mbau caddero in quell'anno pollici 105.16 di pioggia, a Rewa pollici 116.04, ed a Tavuni pollici 101.4; $2^{\circ}$ che la maggior quantitá di pioggia cadde a Mbau in gennaio, a Rewa in aprile, ed a Tavuni in febbraio, e la minore a Mbau in agosto, a Rewa in settembre, ed a Tavuni in gennaio; $3^{\circ}$ che la massima temperatura a Mbau fu di $36^{\circ} 3 \mathrm{C}^{\circ}$ in marzo, ed a Rewa $29^{\circ} 3 \mathrm{C}^{\circ}$ in novembre, e la minima di $18 \mathrm{C}^{\circ}$ a Mbau in luglio, ed a Rewa di $20^{\circ} 2 \mathrm{C}^{\circ}$ in giugno.

Il barometro oscillò durante il 1879 tra $762 \mathrm{~mm}$ e $756 \mathrm{~mm}$.

1 Osservatorio a metri 1856 dalla spiaggia ed elevato metri 23.50 dal mare.

$\begin{array}{llllllll}2 & \text { id. } & \text { id. } & \gg & \text { id. } & \text { id. } & 45 & \text { id. } \\ 3 & \text { id. } & \text { id. } & 91 & \text { id. } & \text { id. } & 9.14 & \text { id. }\end{array}$


III.

\section{Flora e Fauna.}

Quando nel 1859 il Colonnello Smythe venne inviato alle Figi, per esaminare la questione della spontanea cessione che Re Thalombau voleva fare del suo reame all' Inghilterra, lo accompagnò l'egregio botanico tedesco B. Seeman, con l' incarico di studiare e riferire sui prodotti naturali dell' arcipelago.

Lo scienziato ne ritornò con un libro descrittivo e narrativo, in cui aveva raccolto quanto fin' ora si sa di quel popolo, che prima di lui era quasi sconosciuto, e con un ricchissimo erbario, che gli servì a scrivere la sua Flora Vitiensis, opera che lo ha reso illustre, e nella quale sono magistralmente descritte e disegnate le nuove piante da lui rinvenute e introdotte nel patrimonio della scienza.

Egli che aveva espresso un parere contrario a quello del Colonnello Smythe, e precisamente per tale ragione, non potè pubblicare i suoi lavori che dopo qualche tempo e con gravi suoi sacrifizi pecuniari.

Oggi questi lavori sono interamente nel dominio della pubblicitá, e noi prendiamo da essi ad imprestito alcune considerazioni sulla flora figiana. ${ }^{1}$

Le isole sopravento Lakemba, ecc., abbondano specialmente di pandanee ( $P$. odoratissimus e caricosus) e di casuarina (C. equi-

1 Viti or Figian Island di B. SeEman. - Il fondo di un altro libro sulle Figi, di un ufficiale della marina inglese, che porta un nome italiano, RICCI, One New Colony of Fiji, è tolto completamente dal libro del Seeman, diventato raro. Anche la signora Smythe pubblicò un volume sull' istesso argomento. 
setifolia, legrno ferro); la vegetazione minore è costituita da felci dalle foglie dure, che preferiscono le contrade aperte. Questa vegetazione indica un suolo piuttosto povero. Sarebbe però erroneo applicare la stessa regola al lato di sottovento delle isole maggiori, dove esse sono pure abbastanza abbondanti, non perchè il suolo sia troppo povero per sopportare una densa vegetazione erbacea o forestale, ma perchè l'aria è priva di quell' eccessiva umidità lasciata dai venti costanti sulla costa S. E., ed il paese è quindi meno visitato dalle pioggie.

La generale fisionomia della Flora presso la costa è decisamente tropicale: felci arboree, erbe ramose, sei o sette specie di scitaminae, orchidee, epifitte, felci, ecc., pienamente comprovano questo fatto.

Intieri distretti però posseggono un aspetto strettamente sudaustraliano dovuto alla presenza di due acacie e fillodii (Acacia laurifolia e Richei), due Casuarine e parecchie specie di Metrosideros con fiori gialli o scarlatti, un Rubus rampicante, degli Smilax, dei Geitonoplesium, delle Flagellaria, come pure per il particolare abito di varie altre specie.

Le rizofore sono limitate alle bocche dei fiumi, donde la totale mancanza di febbri palustri maligne.

Vi è un piccolo cambiamento di vegetazione quando si giunge a circa 2000 piedi di elevazione, dove le piante peculiari alla costa sono sostituite da forme piu montanine, Mirtacee, Melostomacee, Lauracee, Epacridacee e Vacciniacee, che aggruppate in macchie formano il fondo; orchidee scarlatte, astelie, felci delicate, muschi e licheni, crescono ovunque sui rami.

Nessuno dei picchi finora esplorati ha ancora mostrato una vegetazione veramente alpina; erbe perenni formano delle masse cespitose e dei bassi cespugli generalmente adorni di grandi fiori gaiamente colorati.

La natura è stata invero molto prodiga nella distribuzione de' suoi tesori vegetali in queste isole; ed il Governo della giovane colonia, ben conoscendo che l'avvenire del paese è tutto 
agricolo, ha rivolto speciale cura agli sperimenti e alla diffusione di quelle industrie agricole dei paesi tropicali, che si possono introdurre nell' arcipelago. Con questo scopo molto pratico è sorto un giardino botanico sotto la direzione del già mentovato onorevole I. B. Thurston, cotanto benemerito del progresso che le Figi hanno fatto in cosi breve tempo.

Il nostro dottore Rho, in una breve visita che vi fece accompagnato da una guida indigena, favoritagli dallo stesso signor Thurston, e da un marinaio del bordo, ne riportò vive impressioni che, tolte dal suo taccuino, mettiamo in luce con tutti i loro colori:

«.... Esso occupa una grande estensione ad un miglio e mezzo dall'abitato e ci si va per una bella strada che costeggia la marina. Le coltivazioni cominciano da mare e si stendono sul colle lasciando qua e là alcuni tratti incolti, in cui la foresta vergine è appena tagliata da uno stretto sentiero. Vidi lá coltivate varie specie di caffè, di chinchona, di cotone, di thè, di cacao, molti frutti dell'America Centrale e delle Indie, la noce moscata, il garofano, la salsapariglia, il guaiaco, la vainiglia, ecc. e alcune delle palme che sono piu utili all'uomo. Un Ficus gigantesco, molto rassomigliante al Ficus indica, segnava a monte il termine del giardino, e di lá si entrava in una valletta che la guida mi assicurava esser ricca di felci. Per invogliarmi ad entrare, un magnifico esemplare di Platicerum alcicorne spiegava sul tronco di quell'albero annoso le curve eleganti delle sue fronde vagamente frastagliate come le corna di un alce o di un daino. Dopo esserci arrampicati un po' pel ripido sentiero, arrivammo nel mezzo del bosco al promesso ferns gully, in cui gli alberi di alto fusto scemavano alquanto per lasciar posto ad una macchia di felci arboree, che albergavano alla loro ombra un popolo di minori abitatori della stessa famiglia.

«Quei tronchi svelti e sottili disegnati a figure geometriche e sormontati da un ombrello dal colore smeraldo, quelle felci minori, di un verde smagliante, e finamente frastagliate come un mer- 
letto, mi rappresentavano in piccole proporzioni un paesaggio delle passate epoche geologiche, quando le piante crittogame regnavano sovrane sulla terra specchiandosi nell'onde in cui

$$
\begin{aligned}
& \text { Vagavano i nautili, } \\
& \text { I murici a schiera, } \\
& \text { E l'uomo non era. }
\end{aligned}
$$

«Poco lungi, nella pace romantica e inconturbata di quel paesaggio silvano, un piccolo cimitero accoglie le spoglie dei primi coloni del distretto.

«Io mi diedi a raccogliere felci fra i cippi anneriti di quelle umili tombe e ne radunai in breve ora una discreta quantitá. Noterò fra le più belle l'Hymenophyllum demissum, parecchi Asplenium, fra cui i'A. Richardi e l'A. Hookerianum, che vidi coltivati e meritamente in parechi cottages, un Leptopteris molto facile a scambiare con un Hymenophyllum ed infine un bellissimo Trichomanes, T. reniformis con foglie molto larghe a foggia di rene, come indica il nome.

«E la raccolta sarebbe stata più abbondante se certi nuvoloni accompagnati da un sordo brontolio non ci avessero ricordato l'ora tarda e la probabilità di un acquazzone. Nel ritorno non si prese piu la via della marina, ma la strada che segue la cresta dei colli. Di lassú si godeva una vista stupenda della baia con la sua linea di frangenti su la barriera corallina, la Caracciolo e i pochi altri bastimenti; a destra la punta boscosa che nasconde il villaggio indigeno di Lami; in basso una conca graziosa formata dal giardino; e a sinistra la china dolce, su cui sono sparse, come un branco di pecore fra gli alberi, le bianche casette di Suva. La pioggia, che cominciava a cadere, formava un telone scuro, in un angolo della scena, che aggiungeva alla sua bellezza, ed un pittore ne avrebbe cavato dei begli effetti di luce e di colore. Ma l'artista non mancava, poichè su l'orlo della strada trovammo, seduto nel fango e mal riparato dall'ombrello, un amante delle bellezze campestri, che, con flemma tutta inglese, si sforzava a riprodurre sulla carta la scena che avevamo davanti agli occhi. 
«La pioggia non ci lascio fino alla banchina, ma non per questo la passeggiata fu disaggradevole e sarebbe bastato a ripagarci della fatica l'aver visto una delle piu belle palme che crescono nelle isole del mare del Sud, la Pritchardia pacifica, descritta per la prima volta dal Seeman ».

Dall'attraente e nobile aspetto della palma, che il dottore Rho aveva ammirata, furono colpiti i figiani come gli altri popoli della Polinesia, e tanto, che per preservarla da mani profane, la misero ovunque sotto l'egida della legge del tapir. Le sue grandi foglie, a guisa di ventaglio, privilegio dell'aristocrazia, vengono adoperate solamente a coprire le capanne e le tombe de' Capi. Un'altra palma scoperta dal Seeman nelle Figi è il Sagus Vitiensis, del quale i nativi ignoravano le proprietà nutritive, eccezione notevole alla perspicacia dimostrata da questi popoli nell'osservare e saper trarre ogni utile, specialmente, dal regno vegetale.

Conoscevano dessi, da lungo tempo, anche le virtí medici. nali di alcune piante e le proprietá venefiche di altre, che poche famiglie monopolizzavano, con ufficio di guarire, adoprando le prime, e di uccidere, valendosi delle seconde, se così piacèva ai Capi.

Il talento osservativo e pratico degl'indigeni trova riscontro nel loro calendario, riferito dal Seeman, e che, riproducendolo, non esitiamo a dichiarare, mutatis mutandis, molto piu logico di quello inventato dai novatori della rivoluzione francese a sostituire la nomenclatura dell'anno romano:

1 -2. Vulai nerenere: giugno, luglio, mesi rischiarati, quando la terra è risplendente di piante e di alberi.

3. Vulai cukicuki: agosto, quando i campi d'igname sono in corso di coltivazione.

4. Vulai vavakadi: settembre, quando si piantano le canne per l'igname acció esso vi si arrampichi.

5. Vulai balolo lai lai: ottobre, quando i baloli (Palolo viridis, Gray), un rimarchevole anellide, fanno la loro prima apparizione in piccolo numero. 


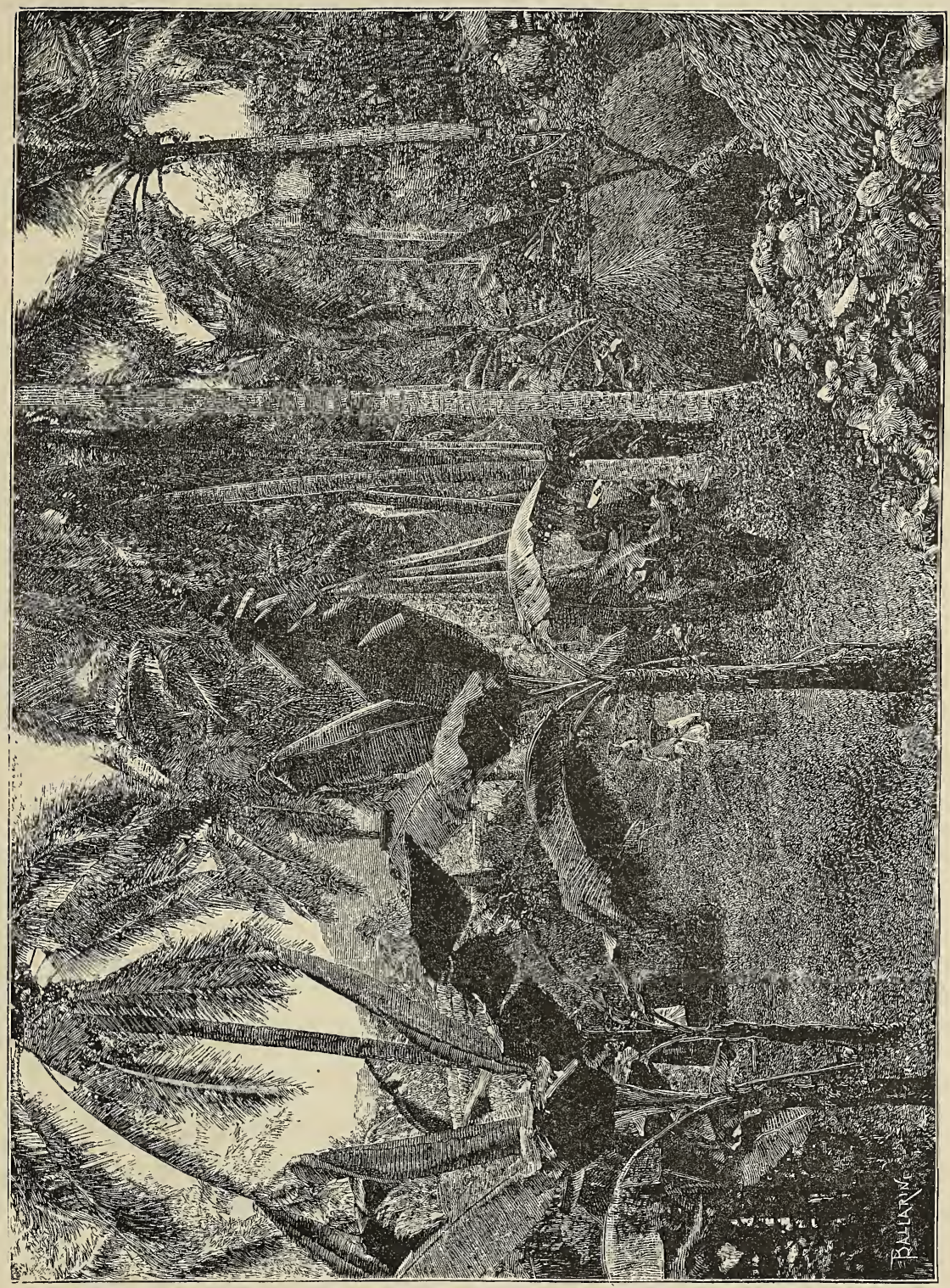



6. Vulai bal̀olo levu: novembre, quando i baloli si vedono in gran numero.

7. Vulai nuqa lai lai: dicembre, quando un pesce chiamato nuqa compare in frotte isolate.

8. Vulai nuqa levu: gennaio, quando i nuqa arrivano in gran numero.

9. Vulai sevu: febbraio, quando si offrono le primizie d'igname ai sevu (ai preti).

10. Vulai kelikeli: marzo, quando si scavano gl' ignami e se ne conservano provviste nelle capanne.

11. Vulai gasau: aprile, quando le canne cominciano a rigermogliare.

12. Vula $i$ doi: maggio, quando fiorisce il Doi (Alphitonia sizyphoides), albero molto abbondante nell'arcipelago.

Gl'inglesi, da colonizzatori pratici, nell'occupare un paese nuovo, prima d'ogni altra cosa ne fanno studiare la mineralogia e la flora, le quali possono essere di un' utilità immediata. La fauna vien sempre per ultima, e quasi sempre è raccolta ed investigata da pazienti e colti dilettanti. La colonia figiana è troppo giovane per essere bene studiata sotto questo punto di vista, sicchè molte lacune restano a colmarsi.

Quanto ai mammiferi indigeni, l'arcipelago non è più ricco che le isole della Società. Tutti o quasi gli esistenti furono recentemente importati, come di leggieri si scorge dalla nomenclatura indigena. Asa, ose, sipi, ecc., sono l'Ass, l'Horse, lo Sheep, ecc. degl' importatori inglesi.

Si conoscono finora 92 specie di uccelli; ritroviamo qui l'Anas boschas ed alcune congeneri; dei piccioni, fra i quali il rimarchevole Diduneulus strigirostris dal becco ad uncino come un uccello di preda, che sembra segnare un passaggio fra i piccioni e il Dronte delle isole Mascaregne, ove questo animale si è estinto nel secolo scorso. Non mancano uccelli di preda e pappagalli, anzi di essi vi sono parecchie specie, la più notevole delle quali è il Coriphilus solitarius, un tempo ricercatissimo per la gaiezza ed armonia 
de' colori ed ora diventato molto raro. Qualche volta compaiono de' pappagalli albini, cioè di un colore giallognolo uniforme.

Le Figi posseggono un solo ofidio non velenoso, ed un batracio; fra i rettili si deve mentovare ancora il Chloroscostes fasciatus, e alcune specie di ofidi marini, del resto comuni a tutto il mare del Sud, quali sono: il Pelanius bicolor, l'Hemydocephalus e la Vermicella annulatus, che esistono pure nelle collezioni di bordo.

IV.

\section{Conclusione.}

Dalle premesse la colonia figiana si presenta con tutti i caratteri di una gemma non indegna delle molte altre, che adornano la Corona imperiale britannica, gemma che attesta la fortuna dei sassoni nella scelta delle loro colonie. Sono fortunati, perchè hanno agio di fare lunghi e ripetuti esperimenti avanti di vincolarsi, ed opportunità quindi di vagliare, senza precipitazione, ogni vantaggio ed ogni inconveniente; il loro colpo d'occhio inoltre è reso ormai sicuro dal lungo tirocinio.

Le flotte da guerra e mercantili britanniche solcano tutti i mari ed in tutti i sensi, perlustrano, con lodevole costanza, ogni costa, ogni piu nascosto rifugio nautico; e se in tanto operare, un palmo di terra vergine di contatto europeo ferma la loro attenzione, perchè Manchester vi potrà trovare un'occasione propizia per esitare sia pure un unico collo dei suoi manufatti, ovvero ottenerne una manata di materia prima, ecco accorrere sul luogo da tutti i punti dell'impero una schiera di bravi missionari e di viaggiatori, per verificare se vi ha utilità nello stabilirvisi.

Nessuno oserà contrastare codesta occupazione privata, che forse si trasformerà, in seguito, in un'occupazione governativa 
formale; la Gran Bretagna ne ha già fatte tante delle occupazioni, che una più, una meno, non monta, e la diplomazia la guarderá con indifferenza, sapendo bene che l'equilibrio politico mondiale non ne sarà perciò turbato. D'altronde, i dominî dell' impero sono cosi vasti, così sparsi e numerosi, da non lasciare un sol punto del globo - disoccupato dalla razza bianca - che per ragioni di vicinato, se non d' influenze o d'interessi, non dia all' Inghilterra sulle altre nazioni un qualche diritto di preminenza. E poi, laddove sventola la sua bandiera, ivi regnano libertà individuale, libertà economica, tutela de' diritti acquisiti dalla gente civile.

Ma pure la Gran Bretagna è eminentemente conservatrice, il che vuol dire restia ad innovare, dilatando i confini del suo impero; però non sono conservatori i suoi numerosi figli, che in balia esclusiva delle risorse del braccio e della mente, costituiscono quella massa imponente di emigranti, la quale si sparge nel mondo, con la febbre e le necessità del guadagno. Codesti britanni sono quelli che scuotono la madrepatria dalla vita rigida e metodica, e la costringono a guardare fuori di casa, ed a provvedere alla protezione dei loro interessi. D'altronde essi chiedono cosi poco all' Inghilterra: un Governo; e le offrono cotanto in cambio: la loro straordinaria attività; che le resistenze dell'inerzia non tardano ad essere vinte dalla volontà dell'opinione pubblica, a cui sempre ubbidisce la saviezza degli uomini di Stato.

L'Inghilterra riluttante ad ogni ingrandimento territoriale non imposto dalle pressioni dei suoi banchieri, industriali, mercanti, pretende tenacemente che altre nazioni non tocchino mai a ció ch'essa reputó conveniente di non appropriarsi: Io o nessuno, tale è la sua massima in tal caso; ed è massima fondata. Essa non ama avere vicini che potrebbero tosto o tardi, per legge di espansione, diventare rivali o concorrenti temibili, e li avrebbe di certo quando non prevenisse le invasioni straniere in terre suscettibili di rapporti con le sue colonie.

- Con codesto intento di evitare molestie da' vicini, gl' inglesi hanno operato sagacemente incorporando all' Impero l'arcipe- 
lago delle Figi, poichè in siffatto modo spezzarono, per sempre, la catena politica ed economica destinata ad allacciare Tahiti alla Nuova Caledonia.

La colonia figiana è un cuneo che separa, lacerandoli, quei due possedimenti francesi, ed ostruisce a tutto benefizio inglese l'arteria commerciale che li avrebbe rinvigoriti, dopo il taglio di Panamà.

Opiniamo, pertanto, che la vera importanza della fondazione della colonia figiana stia in questo, che l'Australia, la beniamina dell' Impero, non corre piu il rischio di essere stretta, a Levante, da un cerchio di ferro, ma che anzi l'ipotetico cerchio nemico è diventato per lei una formidabile opera avanzata di offesa e di difesa.

Il valore intrinseco commerciale ed industriale del gruppo sarebbe, conseguentemente, a' nostri occhi, del tutto secondario, malgrado il quadro ridente che abbiamo fatto de' suoi tesori economici, quadro rispondente alla realtà.

Il gruppo delle Figi è troppo prossimo all'Australia, e questa colonia è troppo ricca ancora, ha troppo spazio disponibile, troppi allettamenti, perchè l'attività del bianco voglia posporla all'altra, in cui l'attività stessa viene presto meno, sotto gl'influssi del clima, del contatto indigeno, del genere di vita.

Nelle Figi, come in tutti i paesi intertropicali od anche semitemperati, il lavoro del braccio appartiene, per ragione di organismo fisico umano, esclusivamente alla razza locale, alla gente di colore, l'europeo non potendo apportarvi, proficuamente, se non il lavoro dell'intelligenza, diretta ad usufruire il primo. Ora, questo fine non è raggiunto che con la leva del capitale; per cui le grandi intraprese, in numero subordinato alla ricchezza del suolo, sembrano dover essere le sole in grado di divenire rimuneratrici, mentre le piccole coltivazioni, le piccole industrie vivranno sempre tisiche, non offerendo compenso alcuno a chi le esercita di fronte alle infinite privazioni, a cui la località specialissima le assoggetta. ${ }^{1}$

1 Sappiamo di un intelligente e laborioso colono, che recatosi nelle Figi in tempi molto propizi per acquistare terreni e coltivarli, dopo 28 anni di 
Per successione d'idee ci domandiamo in questo punto se la bilancia economica, messo in un piattello il capitale europeo, e nell'altro la produzione del lavoro indigeno, si manterrà sempre in un giusto equilibrio? A priori, fermandoci unicamente sull'apparente reciprocità d'interessi, troviamo una risposta affermativa, ma quando vogliamo considerare un istante i circoli evolutivi delle varie razze, dai quali scaturiscono interessi e bisogni cotanto disparati, constatiamo che la risposta potrebb'essere contraria, che cioè un'azione economica parallela fra il bianco e i'indigeno non puó durare a lungo.

Viene giornalmente asserito che la gente di colore scompare al contatto del bianco, del quale essa non sa assimilarsi che i vizi. Egli è indubitato che codesta scomparsa si verifica, e come esempio modernissimo e convincente, informi l'Australia; ${ }^{1}$ ma che avvenga per causa dell' inoculazione de' nostri vizi, eggli è ciò che a noi pare meno esatto. Crediamo piuttosto che le nostre abitudini, che intendiamo imporre agli indigeni, ed alludiamo alle migliori : lavoro, istruzione, religione, sieno esse la vera cagione del deperimento precoce di quelle razze. Noi le togliamo al loro naturale ambito, obbligandole a respirare un'aria, non confacente ai loro polmoni. Vogliamo incivilirle, e le uccidiamo con il nostro progresso.

Le grandi agglomerazioni d' indigeni, conquistate da' sassoni, ed alle quali, con logico intendimento, fu concesso di conservare i propri usi e costumi, i propri ordinamenti politici e religiosi, come nella penisola indostanica, possono riescire istrumenti efficaci e duraturi di produzione e di consumo nelle mani de' bianchi, ove il giogo non ecceda; ma le collettivitá più ristrette, dominate da' bianchi e soggette incessantemente alla dura sferza

non interrotta residenza in paese e d' indefesso lavoro agricolo e commerciale, riuscì ad accumulare appena un peculio, rappresentato da immobili del valore di lire 50,000. Due mila lire circa annue di economia! magro compenso invero per tanti anni di sacrificio.

1 Non si vedono più indigeni nelle regioni meridionali, ove un secolo fa erano assai numerosi. 
del lucro, a lungo andare si logorano, cadono nell'accasciamento, e deboli, per reagire, si struggono di dolore e di desiderî, e spariscono.

Quest' ultimo potrebb'essere il destino riserbato alla razza figiana, sebbene il Governo non abbia tralasciato mai di usare i temperamenti più razionali ed umanitari per conservarla.

La scarsitá delle braccia indigene si manifestò già da qualche tempo, e si dovè ricercare l'aiuto di lavoranti polinesiani e indiani; oggi non basta piú, e si pensa già agl'immigranti dell' Impero Celeste.

Ma in una guisa qualsiasi, per il momento, le braccia si avranno, e le rilevanti imprese, iniziate dagl' inglesi, fioriranno a conforto de' contemporanei, i quali abbandoneranno ai tardi nipoti le preoccupazioni dell'avvenire.

Esaurito ormai, per noi, l'argomento relativo alle colonie di Tahiti e di Figi, argomento in cui ci siamo studiati di porre a confronto due sistemi diversi di colonizzazione, seguiti da due nazioni maestre nell' arte di colonizzare, non crediamo fuori di proposito lo accennare di volo, come sintesi quasi del concetto coloniale, ai varî caratteri che ci sembra abbiano oggigiorno le lontane colonie soggette al dominio della madrepatria.

Con ciò forse riusciremo a dissipare illusioni, a temperare arrischiati giudizi, e, in ogni modo, avremo soddisfatto il nostro vivo desiderio di concorrere, nella miglior maniera che per noi si possa, a dare un avviamento corretto all'opinione pubblica intorno alla grave quistione coloniale, che impensierisce tutti in questo momento.

Le suddette colonie, le quali hanno per obbiettivo supremo la prosperità economica del paese da cui traggono origine, si distinguono, per impronta o carattere speciale, in colonie militari o politiche, in colonie agricole ed in colonie commerciali. 
Le colonie militari o politiche sono fondate per opera esclusiva del Governo, che a seconda dei fini che si prefigge, deve provvederle de' necessari elementi di funzionamento. Possono essere di natura difensiva, come Aden, che guarda le porte di Bab-el-Mandeb nel Mar Rosso, e serve di prima linea di difesa, ad Occidente, in previsione di attacchi contro i possedimenti britannici delle Indie e dell'Australia. Possono invece rappresentare un semplice centro di futura espansione territoriale, come Assab. Comunque, il loro valore intrinseco non deve desumersi dalla feracità del suolo, bensì dalla loro posizione geografica, dalle loro condizioni di sicurezza nautica e militare e dalla maggiore o minore estensione del loro raggio di azione.

Spesso diventano zone di consumo assai rilevanti, attraendo a sè agglomerazioni dense di ğente, la cui attività è attinta dai bisogni insiti all'impianto ed allo svolgimento delle colonie stesse. E qualora si trovino in vicinanza di territori produttivi, diventano inoltre emporî di commercio. Aden, ad esempio, avente a monte lo Yemen, ricco di caffè, e di fronte il paese dei Somali e l'Harrar, ricchi di bestiame, di caffè, di penne di struzzo, di avorio e di gomme, provenienti questi ultimi generi dai paesi Gallas, ha potuto unire al carattere di colonia militare quello di colonia commerciale. Assab, meno favorito perchè il deserto Dankali lo divide dagli altipiani abissini, e lo divide dall' Harrar la vallata inospitale dell'Haussa, potrà pur tuttavia, con lo Yemen che gli sta dirimpetio, e mercè opportune stazioni commerciali nello interno, acquistare, col tempo, alcuni dei pregi della colonia di commercio, conservando massimamente la caratteristica di colonia politica.

Ove le colonie in parola sieno situate sulle grandi vie marittime, diventano sempre, se dotate di un ricovero nautico, utilissimi scali navali di sosta, di approvvigionamento e di raddobbo. Assab, con la sua vasta e sicura rada, potrà assumere questo secondo carattere. Conviene notare, che appunto per i vantaggi nautici che presenta la rada medesima e per la sua ubicazione geografica, l'espansione territoriale, a cui si alluse in principio, favorirà 
vieppiu l'importanza di Assab quale approdo eventuale, imperocchè, mentre crescerà il movimento delle navi per effetto di maggiore operosità economica, nessun altro punto del Mar Rosso e del golfo di Aden, circostanti ad Assab, offrirà mai requisiti maggiori e piu completi di buono ed ampio ancoraggio. Ma non vi ha dubbio, che se quell'espansione dovesse comprendere mercati marittimi già avviati, lo sviluppo commerciale di Assab procederebbe con passo assai lento.

L'amministrazione delle colonie militari o politiche non puó essere altrimenti che d'indole esecutiva, cioè deve informarsi costantemente a criteri emananti dalla ragione di Stato; e per conseguenza è un'amministrazione obbligata a subordinare le sue vedute, i suoi disegni, le sue decisioni all'impero dei rapporti internazionali, ed a preoccuparsi meno dell'azione economica che si svolge attorno ad essa, chiedendole protezione.

La colonia militare o politica forse di tutte è la più difficile a fondarsi: risveglia gelosie e suscettivitì straniere, può perturbare l'equilibrio politico del momento, e ciò che monta altrettanto, non trova aiuto alcuno nel pubblico interessato a possederla, perchè questi non ne afferra sempre l'utilità, ed il Governo nè può nè deve illuminarlo, per non creare ostacoli insuperabili con rivelazioni premature ed inconsulte.

Ad infinite gradazioni d'importanza si presta la colonia agricola, ossia, essa puó abbracciare un piccolo lembo di terra al pari di un continente, ma richiede, essenzialmente, condizioni di habitat in armonia con quelle del luogo originario della razza colonizzatrice, affinchè questa sia in grado di esercitare, dove ha immigrato, le proprie facoltá fisiche e morali in tutta la loro pienezza.

Le localitá in cui l'eccessivo calore spossa il bianco e gli vieta, sotto pena di danno letale, ogni lavoro manuale, non vogliono essere scelte per l'impianto di colonie agricole. Sono quindi escluse le regioni intertropicali, tranne in quei punti ove l'altitudine determina una climatologia simile a quella delle zone temperate. 
Sarebbe pertanto errore grave il credere la Nuova Guinea, l'isola di Borneo ed altre terre tropicali dell'Oceano indiano propizie alla fondazione di colonie agricole. E non è solamente il clima, che non consentirebbe all'agricoltore bianco di prendervi stanza, è altresì la natura del suolo, sovente ribelle, nei punti meglio esposti, ad una coltivazione metodica e rimuneratrice. Le foltissime foreste, la rigogliosa vegetazione, che ammantano pittoresche colline, non di rado hanno presa quasi superficiale; pochi decimetri di humus bastano ad alimentare le intrecciate radici; al disotto, si rivela subito la roccia nuda e aridissima.

La colonia agricola, allorchè si è estesa su di un vasto territorio, acquista, con l'andare del tempo, i caratteri della colonia commerciale ed industriale, e puó comprendere un giorno in sè tutti gli elementi costitutivi di una nazione. Da varie e molteplici cause può avere ripetuta la sua origine. L'Oceania deve la sua alla deportazione; però l' idea moderna sembra voler respingere l'antico sistema di colonizzazione penitenziaria, il quale aveva per base la colonia agricola, ed ama ascrivere il delinquente ad una categoria speciale di maniaci, che conviene curare in casa propria. Invero, il marchio d'infamia lasciato dal colono colpevole pesava ingiustamente, durante generazioni e generazioni, sugli abitanti della localitá colonizzata, attutendo in essi quel sentimento di rispettabilitá collettiva che la razza bianca ricerca cosi gelosamente, perchè imprime potente vigoria alla di lei azione civilizzatrice.

La colonia agricola ha sempre d'uopo, prima di poter funzionare opportunamente, di un periodo di preparazione alquanto lungo, che deve svolgersi sotto gli impulsi del Governo, a cui incombe l'obbligo di mettere in comunicazione facile il luogo prescelto con la madrepatria.

L'amministrazione avrà impronta economica; e scevra da cure politiche o militari, dovrà far convergere tutti i suoi atti all'incremento graduale de' prodotti indigeni, ed alla migliore e più proficua utilizzazione delle risorse della colonia.

La colonia commerciale sorge dall'iniziativa privata, riposando 
sull'impiego del capitale, che ha per istrumenti di speculazione la produzione ed il consumo. Predomina, nel di lei svolgimento, il lavoro dell' intelletto, per cui il bianco trova il luogo piu appropriato all'impianto di essa nelle terre feraci, popolate da razze inferiori, cioè nella zona torrida precipuamente: il contrario quindi di quanto accadrebbe se si trattasse della fondazione di una colonia agricola.

I popoli industriali, presso cui abbonda il capitale, sono quelli che meglio riescono nell' intento, per attitudini e mezzi maggiori. La colonia commerciale nasce e vive per influsso dello spirito d'intrapresa e di associazione, nessun giovamento ottenendo dall'azione individuale isolata, che a sua volta illanguidisce e si consuma infruttuosamente. Ecco perchè il piccolo industriale, il piccolo commerciante, l'artigiano, costretti, se isolati, a livellarsi con gli indigeni, per supplire al proprio sostentamento, si veggono presto ridotti a disertare la colonia commerciale; ed ecco ancora perchè un piccolo numero di coloni bianchi, stretti in associazioni, giunge invece a padroneggiare grandi mercati, nei paesi tropicali.

Tutti i possedimenti europei nelle Indie e nell'Africa furono in principio colonie commerciali, e ne conservano tuttodì i caratteri salienti. In altri tempi la conquista a mano armata, resultato ancora dell'iniziativa privata, ${ }^{1}$ ergeva ed assicurava l'edificio commerciale-coloniale, ma attualmente, mutato il modo, si comperano o si fanno cedere i diritti di sovranità, e si offrono o s'impongono diplomaticamente i protettorati, che sono, in fondo, velate annessioni.

A criteri economici vuolsi ispirare l'amministrazione della colonia commerciale, a fine di spingere sulla via del progresso ragionevole le razze sottomesse, coltivandone i buoni istinti, favorendone la propagazione, e trasformandole in grandi consumatrici e produttrici copiose.

1 Non v'ha chi ignori che le antiche Compagnie delle Indie, britanniche, olandesi, portoghesi, ecc., conquistatrici del suolo in cui dovevano operare, emanavano dal concetto commerciale, sotto l'impulso dell' iniziativa privata. 
Nei territorî d'oltremare scelti, per un possesso permanente, in un paese adatto per clima a soddisfare le necessità fisiche e morali della razza europea, l'obiettivo delle nazioni colonizzatrici fu, in massima, sempre quello di creare nuove provincie, in cui gli immigranti o coloni, col diventare padroni del suolo, potessero accrescere l'energia produttrice della madrepatria, compenetrando, in certo qual modo, l'esistenza sociale di questa con quella della colonia. E fu massima accettata da tutta Europa, sebbene seguita secondo norme diverse in dipendenza dell'indole varia del popolo colonizzatore.

Ma i concetti coloniali direttivi seguirono indirizzi divergenti allorchè si trattò di fondare colonie ne' paesi compresi nella zona torrida, ove era indispensabile avere una massa indigena, in difetto di una massa europea.

L'Inghilterra, riflessiva, nemica di qualsiasi trasporto di sentimentalismo non subordinato alla stretta ragione, e sussidiata dalla scienza, che assegna ad ogni razza un circolo di evoluzione proprio, od in altri termini, una civiltà speciale, volle che la classe dirigente fosse rappresentata esclusivamente dalla razza bianca. Per mantenerne poi il prestigio ripudiò ogni idea di promiscuità fra sassoni e indigeni, i quali ultimi considerò soggetti all'impero assoluto della sua azione regolatrice.

La Francia, invece, ispirandosi a ciò ch'ella si compiacque, in ogni tempo, chiamare la sua generositá, si prefisse spingere gl' indigeni ad un'assimilazione continua con i bisogni europei, modificando così il corso della curva del progresso assegnato dalla natura alla razza conquistata, e favorendo, per facilitare l'intento della colonizzazione, l'incrociamento del sangue francese con quello indigeno.

Senza dubbio alcuno il regime inglese ha dato i migliori risultati, poichè le colonie britanniche sono salite incessantemente per numero ed importanza, mentre le colonie francesi sono andate ogni dì più perdendo di entità, tostochè, indebolita la forza delle armi conquistatrici, le colonie stesse hanno dovuto fare assegnamento sulla energia della loro organizzazione. 
Tuttavia, occorre aggiungere che la Francia non mancò, in passato, di rivelare senso pratico in parecchi de'provvedimenti presi nello impianto delle sue colonie, come appare dalla seguente nota.

Nel secolo xvin, sul principio dell'impianto di una colonia francese, grandi Compagnie anonime, dette di colonizzazione, ricevevano mandato dal Re di esercitare la sovranità sulla terra occupata, e di far funzionare i servizî pubblici; indi, quando la colonia, stante la densità della popolazione francese, possedeva sufficiente vitalità economica, l'amministrazione governativa si sostituiva all'intervento politico della Compagnia. Al Governatore, coadiuvato da un Consiglio superiore della colonia, era conceduta una grande latitudine di azione, anche in materia legislativa, per formulare regolamenti di ogni genere rispondenti ai bisogni locali. Gli impiegati francesi, nel recarsi nella colonia, avevano dinanzi a loro la prospettiva di diventare presto proprietari di beni immobili, e, in breve, acquistavano una grande competenza circa il modo di provvedere agli interessi de' coloni, sentendo di doverli curare gelosamente, dacchè facevano parte essi medesimi della famiglia coloniale.

Un saggio di colonizzazione commerciale modernissima lo si ha nell'atto promulgato dalla Corona britannica nel 1881, con cui viene riconosciuta la costituzione della North Borneo Company.

Questa Compagnia, che nacque per iniziativa di sei sudditi britannici: mercanti, membri del Parlamento, ammiragli, fece acquisto, mercè pagamento in danaro, nel 1877, dai Sultani di Brunei e di Soloo, nell'isola Borneo, di un tratto di territorio situato al N.O. dell'isola stessa, ed ottenne da que' Sultani la cessione di ogni loro diritto di sovranità sul suolo da loro venduto.

Essa, per potere svolgere, in tutta la sua pienezza, l'intento economico, chiese alla Corona talune facoltà di azione amministrativa e politica, che le furono concesse sotto speciali condizioni. Le principali, quelle cioè, che danno alla Compagnia la sua caratteristica impronta, sono: che la Compagnia debba sempre essere e rimanere britannica d'indole e di domicilio; che tutti i 
membri del suo Consiglio direttivo ed i suoi rappresentanti più autorevoli sieno nati nell' impero o naturalizzati inglesi; che non possa trasferire, in altre mani alcuna delle concessioni ottenute senza il consenso del Governo. inglese; che in caso di dissensi tra la Compagnia ed i Sultani di Brunei o di Soloo, il Governo della Regina sia l'unico giudice ed arbitro; che la Compagnia debba, con ogni più valido mezzo, far cessare la schiavitu sul territorio da lei amministrato, e vietare qualsiasi ingerenza nella religione professata dagl' indigeni od altri; che, nell'amministrazione della giustizia, la Compagnia tenga il massimo conto degli usi, costumi e leggi del paese; che la nomina del primo rappresentante, in Borneo, della Compagnia sia sempre sottoposta all'approvazione di S. M. la Regina; che la Compagnia non monopolizzi mai il commercio, tuttochè possa imporre tasse doganali ed altre, per provvedere alle spese di utilità pubblica.

Fuori della giurisdizione della madrepatria ed assoggettate a leggi straniere, esistono parimente le tre categorie di colonie suenunciate, e l'Italia ne conta parecchie nelle Americhe; ma non è qui il luogo opportuno per discorrerne.

Piuttosto ci faremo a chiederci, prima di ultimare, se sia veramente un bisogno odierno ed assoluto, per gli Stati europei, quello di possedere colonie.

Per noi l'affermativa emerge dallacostante legge dei compensi: gli Stati esuberanti di vitalitá, afflitti, gli uni, dalla pletora della produzione, gli altri, dall'eccesso del consumo, sono naturalmente indotti a ricercare condizioni diverse di svolgimento economico, e le trovano occupando nuovi territori e colonizzandoli. In questa ricerca, l'esito migliore appartiene al piü previdente, o al piu forte, od anche al più audace.

Forse non mai, come oggi, si ripresenterà un'occasione così propizia per soddisfare necessità di espansione territoriale, poichè forse non mai si verificherà nuovamente il caso che queste necessità sieno sentite, con prepotenza, e per così dire, all'unisono, da molti Stati, ed in modo che le esigenze di ciascuno e le conces- 
sioni reciproche possano pareggiarsi senza gravi perturbazioni politiche.

Qualora eventi inaspettati non cambino i destini politici delle terre indiane, l' Italia dovrà rimanere inerte in quelle parti del mondo, rispetto alla sua azione coloniale; e cosi pure in Oceania, ove ogni isola, ogni arcipelago conta già un padrone, più o meno dichiarato; però potrebbe, chi sa? avvicinandosi alle coste americane del grande Oceano, ottenere ancora, mercè accordi preventivi, il possesso di qualche isola dell' arcipelago di Galapagos, ${ }^{1}$ abbandonato o quasi dal suo sovrano: la repubblica dell' Equatore.

Ma l'Italia ha dinanzi a sè e presso di sè un continente vastissimo: l' Africa, che le mostra la via piu immediata per espandersi, per affermare la sua esistenza di grande nazione, e che aspetta anche da lei il soffio rigeneratore della civiltá.

1 Vedere nella parte intitolata Isole tropicali del mar Pacifico. 
ISOLE TROPICALI DEL MAR PACIFICO 



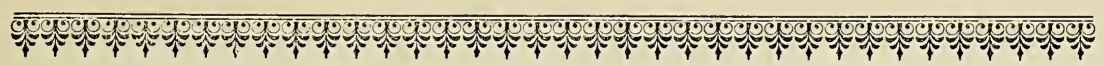

ISOLE TROPICALI DEL MAR PACIFICO

Tahitr e le Figi, mercè la loro posizione geografica e le loro speciali condizioni di governo, possono considerarsi quali centri di attrazione economica politica de' numerosi gruppi di isole, che il mar Pacifico, lungo la fascia tropicale, racchiude fra l'America, l'Australia e la Nuova Guinea. Quindi, l'importanza di tali gruppi deve necessariamente entrare in linea di calcolo con quella che appartiene a Tahiti ed alle Figi; ed il non farne menzione, dopo aver parlato di questi due centri, equivarrebbe a nascondere una parte interessante di un tutto, che vuol essere mostrato sotto i suoi varî aspetti.

Noi che ci siamo prefissi portare ovunque la maggior luce, dentro i confini, s'intende, assegnati a questo nostro piccolo studio, non ristaremo dal dare contezza in forma di appendice ed in modo sommario, de' gruppi succitati, attingendo i particolari ad essi relativi a' documenti più recenti ed attendibili. ${ }^{1}$

1 Molti di questi documenti, che sono tuttora inediti, figurano nel Report of the proceedings, of the intercolonial Convention - 1883, New South Wales (Australia), favoritoci dall' egregio Premier dell'ora detta colonia, M. Stuart. 
ISOLE TONGA O DEgLI AMICI.

L'arcipelago delle Tonga conta almeno un centinaio d'isole e d'isolotti. Le isole Tonga-Tabù e Vavao hanno ciascuna, in lunghezza, una estensione, che varia fra le 15 e le 20 miglia. Le isole Lette, Tofua, Kao, Nanauka, Lefuga, Eoa si estendono, in lunghezza, da 5 a 7 miglia; le rimanenti sono assai più piccole, e parecchie non sono che banchi di sabbia o di corallo coperti di cespugli. Tofua, Kao, Lette e i due scogli Hunga-Hapai e HungaTonga, per la loro elevazione, possono essere riconosciuti, dal largo, a 15 o 20 leghe di distanza. Eoa, Nanauka e Vavao sono di moderata altezza; TongaTabù e le altre isole sono molto basse.

Il gruppo è diviso in tre stazioni di missionari protestanti, che hanno per sede Tonga-Tabù, Hapai e Vavao.

La popolazione ascende a circa 10,000 anime, convertite tutte al cristianesimo.

Un Re indigeno governa l'arcipelago delle isole Tonga, con l'assisténza di un'Assemblea composta da' Capi tribù.

Gl' indigeni coltivano l' igname, la patata dolce, la banana, il cocco, l'albero del pane, la canna da zucchero, il ti (Spondias dulcis). Il pandano abbonda nel gruppo. I missionari v'introdussero l'arancio ed una specie di Aunona.

Sotto l'aspetto botanico l'arcipelago delle Tonga rassomiglia perfettamente a quello delle Samoa.

\section{IsOle SAMOA O DE' Navigatori.}

Samoa è il nome indigeno del gruppo de' Navigatori, che per aspetto, varietà e bellezza può dirsi incantevole. Le isole sono di origine vulcanica, mostrando varî crateri; dieci di esse sono abitate.

L'isola maggiore si chiama Savaii; la sua lunghezza è di 48 miglia; ne ha 22 in larghezza e 150 in circonferenza. Comprende una catena di monti, il più elevato de' quali s'innalza a 1220 metri dal pelo dell'acqua; in quel punto si trova un gran cratere.

Tutuila è di tutte le isole la più bella; è lunga 17 miglia, larga 5 , con una circonferenza di 60 miglia.

Savaii ed Opolu contengono la maggior estensione di terreno piano; due terzi del quale - 200,000 acri - sono molto adatti per la coltivazione.

Tutuila è più montuosa di Savaii ed Opolu, e probabilmente un terzo sol- 
tanto della sua superficie - circa 20,000 acri - è coltivabile; essa però ha sulle dette isole il gran vantaggio di possedere un eccellente porto.

Il clima delle Samoa è dolce ed aggradevole; la temperatura colà varia tra $22^{\circ}$ e $2 \gamma^{\circ} \mathrm{C}^{\circ}$; il caldo vi è mitigato dalla brezza, che non manca mai di soffiare. Gli alisei vi si mantengono stabili da aprile ad ottobre, e soffiano con 'maggior forza in gennaio e luglio. Da novembre a marzo i venti spirano con frequenza dall'O., ma sono di breve durata.

Alcune volte forti colpi di vento si fanno sentire in gennaio. In febbraio il tempo è bello; i venti sono variabili. Marzo è il peggiore dei mesi, perchè il più incostante; si hanno allora forti raffiche dal N. e dal N. O. Da dicembre a marzo pioggie abbondanti. I mesi di giugno e luglio sono i più freschi, quelli di settembre ed ottobre i più caldi.

Raramente l'arcipelago è visitato da uragani. Se ne ebbe uno nel 1840, un altro nel 1850 ed un terzo vent'anni dopo, nel 18\%0. Essi sono, per lo più, localizzati; infatti, l'uragano del 1870 si scatenò soltanto sull' isola di Tutuila, e non si estese al di là.

I principali prodotti dell'arcipelago consistono in noci di cocco, cotone, noci-candele, banane, aranci, limoni, patate dolci, tamarindi, albero del pane, canne da zucchero, indaco, arrowroot, zenzero, caffè, tarò, ignami, ecc.

Il genere principale di esportazione è il copra.

Nel 186\%, il valore complessivo delle importazioni ed esportazioni ascendeva a lire sterline 77,198 , e nel 1871 a 70,000 . Dal 1867 al $18 \% 1$, il movimento delle navi fu di 14,282 tonnellate. I generi importati provennero dall'Australia e da Amburgo, sebbene in maggior parte di manifattura inglese.

Nel 1858, gli affari commerciali dell'arcipelago erano nelle mani di due mercanti inglesi, di una ditta americana degli Stati Uniti e di una ditta tedesca. Nel 18\%, sei mercanti inglesi erano già stabiliti in Apia, oltre un certo numero di piccole agenzie; una ditta tedesca, con parecchie stazioni ed agenzie; e tre ditte americane, anch'esse con agenzie.

Ragguardevoli estensioni di terreno furono comperate dai residenti stranieri; più di 28,000 ettari, al prezzo. variabile da 1 a 4 lire sterline l'acre (ettari 0.40).

Come negli altri arcipelaghi tropicali, in quello delle Samoe prevale l'elefantiasi, da cui non vanno esenti i bianchi. Numerosissime vi sono le mosche e le zanzare.

Gl' indigeni samoesi sono una bella razza: alti, ben proporzionati, e di color bruno chiaro. Hanno ottimo carattere: docili, fidi, ospitalieri, pieni di vitalità, vivaci. Conversando tra di loro o con bianchi sono assai cortesi e bene educati. Usano varie espressioni di rispetto a seconda del rango sociale del loro interlocutore; ad esempio, nello indirizzarsi ad un gran Capo o ad 
un europeo di qualità dicono: Lautểo, ossia vostra maestà; parlando con un capo di lignaggio inferiore: Lan-Susu, vostra signoria; con Capi del più basso lignaggio: Ala-Ala. Il saluto consueto fra persone dello stesso rango è Omai o Sau, che significa: Siete arrivato? Siete qui?

Solamente gli uomini si tatuano, e non sul viso, a somiglianza dei nuovi zelandesi, ma soltanto sul corpo, dalla cintola al ginocchio, e di nero. Ad una certa distanza, il tatuaggio fa credere ch'essi abbiano indossato calzoni corti.

Gl' indumenti indigeni si riducono ad un pezzo di masi o di calicò fermato attorno ai fianchi. Alcune donne si coprono il seno con due fazzoletti disposti in forma di pianeta. Nelle foreste, oppure al lavoro del campo o a quello della pesca, semplificano il loro abbigliamento limitandolo ad una foglia.

Fabbricano una certa qualità di stuoia finissima con la fibra di una pianta chiamata lau-ie, che ha grandissimo valore, ai loro occhi, e che preferiscono a qualunque stoffa europea per ricca che sia. La usano nelle occasioni solenni, e più è vecchia, più è apprezzata; alcune hanno una vera celebrità. La più antica, chiamata moe-e-fui-fui, ossia la stuoia del sonno fra i rampicanti, conta oltre due secoli. Le migliori stuoie si fanno a Manuia.

Uomini e donne dedicano gran parte del tempo a pettinarsi; incalcinano sovente i capelli, i quali diventano poi di colore fulvo; amano decorarli con fiori.

La natura provvidissima non impone agl'indigeni alcun faticoso lavoro per sostentarsi.

Le loro case sono pulite, di solida. struttura, generalmente circolari, con il tetto conico elevatissimo e sostenuto al centro da forti puntelli.

I samoesi sono molto abili nella manovra delle loro piroghe, di cui hanno cinque tipi diversi: a-lia, doppia canoa, che può portare fino a 200 nomini; tau-mua-lua, di 10 a 15 metri di lunghezza: va-alo, piroga da pesca con contrappeso; e paopao, piccolissima piroga per una sola persona.

Gl' indigeni sono ormai convertiti tutti al cristianesimo. Il primo missionario che introdusse la religione cristiana alle Samoa fu il reverendo wesleyano J. Williams, nell'agosto 1830. Attualmente vi è ad Apia un vescovo cattolico; ed un certo numero di preti si trova sparso fra parecchie isole dell'arcipelago. La popolazione indigena ascende a circa 34,000 abitanti.

Nessun samoano ha mai voluto emigrare, per fare l'agricoltore.

In passato vi era un Tui-Somoa, o Re, di tutto l'arcipelago, simile al Tui-Tonga ed al Tui-Viti (Re di Tonga o delle Figi), ma da cento anni circa non esiste più un vero Re, ogni città o villaggio godendo della propria autonomia sotto il governo di un Capo proprio. Non di rado alcuni villaggi si costituiscono in confederazione, ed allora il Capo più influente esercita 
il potere supremo. I Capi di villaggi o città sono chiamati alii, dopo i quali vengono i capi delle famiglie più influenti di ciascuna città detti tu-la-fale. Ogni affare che interessa il villaggio è discusso dal Consiglio dei tu-la-fale, presieduto dall'alii. Le decisioni del Consiglio diventano leggi per l'intero villaggio. Il sistema di governo varia in molti distretti; in alcuni i matuas, o patriarchi, esercitano considerevole autorità, mentre in altri l'autorità maggiore appartiene ai $t u-l a$-fale, ma però collettivamente e non individualmente.

Non ha guari si annoveravano tre Capi principali, che prendevano il titolo pomposo di Tui (Re), - Tui-manua, Tui-ana, Tui-atua -, sebbene la loro autorità non oltrepassasse quella di Capo di città, essendo soggetti al Consiglio degli anziani del paese.

Durante alcuni anni, la Germania e gli Stati Uniti, per mezzo de' loro rispettivi consoli, partitamente o di concerto, esercitarono molta influenza sui pubblici affari, ossia una specie di controllo. In quest'ultimo tempo l'Inghilterra e la Germania riconobbero formalmente, in seguito ad un trattato conchiuso col Re ed il Governo di Samoa, la completa indipendenza dello Stato samoano.

Apia, nell'isola Upolu, è la residenza della maggior parte de' coloni europei e quindi de' consoli.

\section{ISOLE COOK.}

È un gruppo di nove o dieci isole assai lontane l'una dallaltra, che, in complesso, è poco importante. Mangaia e Rarotonga, di origine vulcanica, sono le più notevoli per altezza, estensione e popolazione. Atui, Mitiero e Mauki o isola di Parry sono banchi di corallo alti 3 o 4 metri e coperti di vegetazione.

\section{Nuova Caledonia.}

La Nuova Caledonia è possedimento francese destinato a deportazione. In lunghezza è 200 miglia, in larghezza 30 . La sua popolazione si fa ascendere a circa 60,000 anime. Di origine vulcanica, è traversata, nel senso della sua lunghezza, da N.O. a S. E., da una catena di monti, i quali, in alcuni punti, raggiungono l'altezza di 2438 metri; ed è circondata da una barriera di banchi di sabbia e di corallo. Nel N. E. vi ha il porto di Balade, sicurissimo, e nel S. O. dell'isola il porto di Saint-Vincent, ugualmente sicuro.

Gli indigeni sembrano di razza papuasiaca; sono divisi in tribù, alcune delle quali antropofage. 
Le colonie australiane fecero recentemente vive istanze al Governo della madrepatria affinchè, con l'adozione di opportuni provvedimenti, paralizzasse le serie conseguenze, che esse temono della legge francese circa la deportazione di criminali nella Nuova Caledonia e sue dipendenze.

La colonia di Queensland, in particolare, si lagnava che il Governo della Nuova Caledonia oggi non domandi più l'estradizione dei deportati evasi da Numea; domanda senza la quale le autorità coloniali non possono procedere contro i deportati stessi.

Siccome la deportazione nella Nuova Caledonia tende, da parte della Francia, a sbarazzarsi della peggior classe dei suoi delinquenti, che, messo piede a terra, diventano liberi, così quell' isola diventa per le colonie australiane una sorgente costante di grave pericolo.

ISOLE DELLA LEALTÀ.

Queste isole possono considerarsi come parte integrante del gruppo della Nuova Caledonia, poichè corrono parallelamente ad essa ad una distanza di 50 a 60 miglia. Si chiamano Marè, Lifu, Nea; fra le due prime vi sono cinque isolotti.

NuOve EBRIDI.

È un gruppo che annovera le seguenti isole: Aneiteuma, Tana, Erromango, Vate o isola di Sandwich, Api, Ambryna, Aurora, Lepers, Mallicollo e Spirito Santo. Quest'ultima, la maggiore, ha la lunghezza di 22 leghe e la larghezza di 11. Mallicollo, che vien dopo per estensione, iè lunga 18 leghe e larga 8. Erromango acquistò, in passato, una certa notorietà per il massacro del colto e benemerito missionario protestante Williams.

Aneiteuma ha una popolazione di 2200 anime, che tende a diminuire. Gli indigeni sono tutti cristiani, ed ogni individuo al disopra de' cinque anni legge più o meno e frequenta le scuole. Non v'ha traccia alcuna, nell' isola, di cannibalismo; la sua costituzione fisica è vulcanica; il clima è umido ed alquanto malsano; gli uragani sono frequenti e violenti. L' isola è circondata da banchi di corallo. Vivono in essa alcuni bianchi.

Tana conta una popolazione di 10 a 20 mila abitanti, il cui livello morale, in passato assai basso, va decrescendo sempre più dopo che i bianchi v'importarono armi e malattie. Il cannibalismo regna ancora in tutta l'isola.

Vate, con circa 11 mila abitanti, non ha che un solo villaggio conver- 
tito al cristianesimo, quello di Erakor; gli altri sono pagani e dediti al cannibalismo. 亡̀ soggetta a forti scosse telluriche.

Nelle Nuove Ebridi non vi sono Capi riconosciuti, e poichè gli indigeni si distinguono per lo spirito diffidente e battagliero, cosi non esiste colà sicurezza alcuna per la vita e la proprietà. I bianchi, ne' loro rapporti con gli indigeni, debbono essere molto circospetti e tenersi sempre in guardia, nonostante le apparenze di amicizia, contro inaspettate insidie. Gl'indigeni sono di colore oscuro e di moderata statura; in talune località, come ad esempio nelle isole Spirito Santo e Mallicollo, hanno robustissimo aspetto ed i capelli lanuti. Adoperano, per armi, clave, lance, archi e frecce avvelenate. Le loro piroghe, grossolane di forma e di fattura, sono munite di contrappeso.

I prodotti del gruppo consistono nel frutto dell'albero del pane, cocchi, banane, arrowroot, canne da zucchero, patate dolci, ignami e tarò. In alcune isole si trovano maiali, specialmente a Mai, la cui popolazione è reputata pessima.

\section{Arcipelago di Salomone.}

Si estende da N.O. a S. E. per uno spazio di 200 leghe. È composto di 8 o 10 isole principali e di molte altre più piccole. Le maggiori sono: Bougainville, Choiseul, Isabella, Gaudalcanar, Malayta e San Christoval. Quest'ultima ha 73 miglia di lunghezza e 23 di larghezza nella sua parte più ampia. Malayta è lunga 70 miglia; Isabella ha 120 miglia di lunghezza e 25 di massima larghezza. Non fu accertata ancora l'estensione di Bougainville e di Choiseul, ma si ritiene che la loro superficie superi quella delle isole sunnominate. La struttura generale è dappertutto la stessa: una lunga catena di montagne, spesso bassissime, il cui asse segue la direzione, su per giù, del gruppo. Da un lato, il pendio dei monti inclina dolcemente verso il mare; le terre sembrano generalmente basse e spesso delineate da una cintura di rizofore, bagnata dal mare. Un'attiva e rigogliosa vegetazione ammanta tutto il suolo, e non è che qua e là, a rari intervalli, che lo si può scorgere, sia coperto soltanto da felci ovvero denudato dal fuoco. Le isole principali possiedono tutti i vantaggi delle grandi terre: estese pianure, copiosi fiumi scendenti fra i monti; e, a giudicare dalla robustezza del fusto e dall'abbondanza degli alberi, suolo grandemente ferace.

Gli abitanti di queste isole, comunemente più bassi di statura degli indigeni già citati, sembrano dotati di maggiore energia ed attività di quella che si riscontra generalmente fra le popolazioni della regione oceanica. Le loro piroghe sono graziosissime, leggiere e senza contrappesi; ne possiedono alcune 
grandi, per la guerra, che possono portare da trenta a sessanta guerrieri, e con le quali si spingono talune volte a grandi distanze, fuori della vista di terra. Gli indigeni sono alquanto abili negli intagli, per eseguire i quali adoperano istrumenti fatti con la madreperla.

\section{ISOLE BANKS.}

È un gruppo giacente al Nord delle Nuove Ebridi, tra la latitudine di $13^{\circ} 16^{\prime}$ e $14^{\circ} 10^{\prime}$ Sud, e $167^{\circ} 17^{\prime}$ e $158^{\circ} 14^{\prime}$ Est, Green.

Vanua-Lava, la maggiore isola, ha 15 miglia di lunghezza da Nord a Sud, ed è rimarchevole per i suoi monti arrotondati, il più elevato de' quali, situato nel N. O., misura 854 metri sul livello del mare. Ha due cascate e parecchie sorgenti sulfuree. La popolazione ascende a circa 1500 anime; gli indigeni sono pacifici e socievoli.

Santa Maria, ovvero l'isola Gana, che viene dopo per grandezza ed importanza, ha monti elevati dal pelo delle acque 610 metri, foltamente boscosi, ed è coltivata. Qúando soffia l'aliseo la costa di mezzogiorno e quella di levante sono inaccessibili. Gli abitanti sono litigiosi e cattivi; non di rado attaccano con le loro freccie gli armamenti delle imbarcazioni europee.

Mota o Pane di Zucchero ha circa da 8 a 10 miglia di circonferenza. Conta circa quaranta villaggi, con una popolazione complessiva di circa 2000 anime, senza alcun Capo riconosciuto. Gli indigeni sono buoni, e varî fra essi capiscono un po' d'inglese. Produce frutta, canne da zucchero, tarò, patate ed ignami.

Araa è di tutto il gruppo l'isola più piccola; il numero de' suoi abitanti è fra i 2 ed i 3 mila, d'indole buona.

\section{Isole Santa Crux.}

Questo gruppo è composto di sette grandi isole: Vanikoro, Santa Cruz, Guerta, Volcano, Edgecombe, Ourry e Lord How, oltre varie piccole al Nord ed al N. E. dell'isola Volcanó. Vanikoro è la più meridionale. Agli occhi degli europei appare come la più rimarchevole, non già per la sua estensione o ricchezza, ma per essere stata il teatro della disastrosa perdita delle due navi di La Pérouse, nel 1\%88, erento che non potè essere constatato, con certezza, prima del maggio del 1826, ossia trentotto anni dopo.

Il piccolo gruppo di Vanikoro consta di due isole d'inuguale estensione, di cui la prima non ha meno di 30 miglia in circonferenza, e la seconda non 
più di 9 miglia. Sono entrambe alte e coperte d'alberi fino alla riva. La popolazione è scarsa; il litorale soltanto è abitato, essendo l'interno una densa e selvaggia foresta, quasi impenetrabile.

Santa Cruz è circa 15 o 16 miglia in lunghezza, con una barriera di scogli tutt'attorno, apparentemente innocua; essa isola è foltamente coperta di alberi e vi abbonda l'acqua, che traversando, sotto forma di torrente, i villaggi, si riversa in mare.

Gl'indigeni sono di atletica statura, e si recano sulle navi europee senza esitazione, per vendervi maiali, frutti dell’albero del pane ed ignami, ed anche stuoie confezionate con un certo gusto. La costruzione delle canoe e case è del tutto primitiva. Le canoe sono dipinte di calce e portano un contrappeso; alcune sono doppie e grandi. I villaggi sono estesi e le case circondate da steccati. Dal lato Nord, i villaggi sono prossimi al mare, e contano da 300 a 400 abitanti ciascuno.

Non bisogna fidarsi troppo delle apparenze di bonarietà degli indigeni, giacchè altra volta aggredirono, senz'alcuna ragione; l'imbarcazione di un missionario, ferendo con freccie tre marinai, due de'quali morirono poco dopo. I loro archi sembrano armi formidabili: sono lunghi sette piedi, e le freccie in proporzione.

\section{ArCipelago Delle Luisiadi.}

É situato vicino alla parte S. E. della Nuova Guinea, abbracciando le isole Adele, Roussel, Renard, S. Aignan, De Boyne, Bonvouloir, d'Entrecasteaux e Trobian. Roussel e S. Aignan sono le maggiori del gruppo; quest'ultima ha 27 miglia circa di lunghezza. Tutte le altre sono piccole isole corallifere. L'arcipelago è finora poco conosciuto, ma lo si crede abitato da numerosa gente.

\section{Nuova Brettagna e Nuova Irlanda.}

Due isole collocate fra la parte orientale della Nuova Guinea e l'Equatore. Dumont d'Urville, che le visitò nel 1827, si esprime in questo modo a loro riguardo: «Raramente la natura ha impresso un aspetto cosi delizioso ad un paese vergine della mano dell'uomo, con una varietà di contorni così aggradevole e con effetti di prospettiva così belli. La costa è dappertutto completamente sana, accessibile e bagnata da acque tranquille; la terra si innalza gradatamente ad anfiteatro in varî punti, qua e là ombreggiata da nere foreste, o da meno fitta vegetazione.... I due picchi del monte Glowcester 
coronano questa ridente scena con le loro imponenti masse; le loro maestose vette spesso penetrano attraverso le nuvole. In tutto il lato occidentale, a 12 miglia di distanza, il nostro orizzonte abbracciava le linee ondulate delle isole Rook, le quali formano, con la Nuova Brettagna, lo stretto di Dampier ».

ISOLE DELL'AMMIRAGLIATO.

La magggiore di questo gruppo è l'isola dell'Ammiragliato, il cui centro trovasi a $2^{\circ} 18^{\prime} \mathrm{S}$. lat. e $146^{\circ} 41^{\prime} \mathrm{E}$. Green. Alcune isole del gruppo sono ritenute come fittamente popolate da gente apparentemente contenta e felice. Vi abbondano gli alberi del cocco.

\section{TOKELAN O GRUPPo DELL' UNione.}

Tokelan od Unione è un gruppo che consta di tre agglomerazioni d'isolotti nominate Takaofo, Nukunono ed Atafu. Ogni agglomerazione è collegatà ad un banco corallifero, che forma uno de' numerosi atolli del Pacifico.

Il lagone di Takaofo, che ha più di venti isolotti, misura 8 miglia di circonferenza e 5 di larghezza. La popolazione ascende colà a 223 persone, di cui più di un terzo degli adulti è di sesso femminile.

L'atollo di Atafu rassomiglia a quello di Takaofo, ma è più piccolo. La sua popolazione era un tempo di 136 abitanti.

\section{Gruppo delle lagune (Hellix Islands degl'inglesi).}

Nukulaelae è una riunione di parecchi isolotti coronanti lagoni. Possiede un villaggio oggi abitato da pochi indigeni, che è la località, ove gli schiavisti peruviani fecero, nel 1863, una delle loro maggiori razzie.

L'isola Tunafuti ha un lagone di 12 miglia nel suo maggiore diametro, e 5 o 6 nel suo minore. Gli abitanti coltivano il tarò, la banana, l'albero del pane, ecc., secondo i processi medesimi, che seguono gl'indigeni di Tuamotù, cioè facendo trincee, nel corallo, di due o tre metri di profondità e colmandole con il terriccio, che si forma alla superficie del suolo. Vaiputu è un atollo completamente chiuso. Ha una popolazione di circa 376 abitanti, reputati di indole ottima.

Le altre isole Nukufetau, Nui, Nuitao, Nunomaga, Nanomea non diffe- 
riscono granchè dalle precedenti sia nella loro costituzione fisica, sia nelle loro condizioni idrografiche, sia infine riguardo alla popolazione ed ai prodotti del suolo.

Gli abitanti del gruppo sono una eletta razza d'uomini, veri giganti.

Il $10 \%$ misura od oltrepassa in istatura metri 1.82 , con il torace ben proporzionato. Si debbono considerare tranquilli e pacifici. Di rado nascono dissensi fra loro, e questi, in ogni caso, cessano presto, mercè l'autorità del Re o dei Capi. In alcune isole s'ignora ciò che sia la guerra.

\section{BANCO SOFIA.}

A metà distanza fra l'ultima isola del gruppo delle Lagune (Hellix Islands) e l'isola Rotumath della colonia figiana, nella latitudine $10^{\circ} 50^{\prime} \mathrm{S}$. e longitudine $179^{\circ} 30^{\prime} \mathrm{E}$. Green., le carte inglesi segnano un banco con l'appellativo di Sophia.

Or questo banco ha una storia, a cui si associa il nome italiano.

Era nel 1853; uno schooner a vela, sardo, di 120 tonnellate, comandante il Capitano Agostino Tortello, armatore il Capitano Pietro de Amezaga, avendo posto in salvo - a porto Jackson-Sydney - un equipaggio inglese, in procinto di perire con la propria nave, che piena d'acqua colava a picco in vicinanza della costa, si recava in Cina, passando a levante della Nuova Caledonia e delle Caroline. Pochi giorni dopo la sua partenza da quel porto, lo schooner, favorito dal tempo, era giunto nei pressi di Rotumath, ove navigava con insolita circospezione e cautela.

In quei tempi l'idrografia della Polinesia difettava assolutamente di esattezza: le carte non fornivano che scarse ed imperfette nozioni intorno all'esistenza dei numerosissimi pericoli del mare australasiano, e l'unica guida, ossia la meno incerta, che potesse procurarsi il marino, consisteva nella traccia dei viaggi di Captain Cook, da quelle carte riportata.

Lo schooner camminava adunque dietro i solchi del celebre Capitano, quando verso il tramonto, splendido il cielo e chiarissimo l'orizzonte, la vedetta, collocata nell'alto, gridó: «Vela di 
prua ». Il vento soffiava con una certa forza; la nave era ottima veliera, sicchè, in breve, s' intese un secondo avviso della vedetta stessa: "Frangenti di prua in direzione della vela ». La velatura venne immediatamente ridotta, a fine di poter con più facilità manovrare, e poichè era ancora giorno, la corsa fu proseguita, volendosi presto riconoscere e l'entità e la natura dell' ostacolo sorto così inaspettatamente.

Infatti, non tardó lo schooner ad essere dinanzi ad un atollo, su cui posavano le vestigia antiquate di una grossa nave. La notte era vicina e prudenza consigliò di rimandare al domani ogni ulteriore e piu minuta indagine. Mise intanto sui bordi, prese cioè un'andatura di temporeggiamento. La mattina susseguente un'improvvisa e violenta burrasca lo costrinse a correre con le vele chiuse; ma poche ore di poi l'atmosfera si rischiarò e comparvero nuovamente ali'orizzonte l'atollo e la sua preda. A mezzogiorno lo schooner era a 500 metri dall'uno e dall'altra senza che il maggior calumo di scandaglio accusasse alcun fondo. Alzó la bandiera, sparò vari colpi di cannone, però nulla potè scorgere che indicasse la presenza di gente sull'atollo.

L'equipaggio dello schooner constava di sole nove persone; pur tuttavia, il Capitano aveva già ordinato di mettere una lancia in mare, per assicurarsi viemmeglio che nessun essere umano si trovava a terra; e la spedizione avrebbe avuto luogo, se un'altra burrasca improvvisa ed assai più violenta di quella del mattino non avesse obbligato di riprendere celeremente il largo.

Il temporale persistendo dovè lo schooner rinunziare al suo proposito di far eseguire un'escursione sull'atollo, e contentarsi di essere riuscito a stabilirne la precisa posizione, grazie a rigorose osservazioni e calcoli.

L'atollo aveva circa 2 miglia di lunghezza e mezzo miglio di larghezza: era composto di un banco di sabbia corallifera, di forma triangolare, e di una corona di scogli determinanti il lagone. Sull'estremitá del banco, un centinaio di metri dalla riva, ergevasi la prua, quasi intatta, della nave naufragata con alcune 
parti dell'ossatura dello scafo; due gomene distese verso il mare uscivano fuori dagli occhi della prua medesima dinotando, palesemente, che la nave si era perduta molti anni addietro, nell'epoca cioè in cui non si usavano ancora catene di ormeggio, e che nel trovarsi vicino all'atollo aveva dovuto tentare di dare fondo, per non investire. Una piccola casetta di legno dinotava altresì che l'equipaggio aveva soggiornato alcun tempo sull'atollo.

Queste vestigia erano state una vera provvidenza per lo schooner, chè, chi sa? procedendo nella direzione iniziale, l'oscurità della notte, la persuasione di battere la buona via, e la difficoltà di padroneggiare a piacimento i movimenti della nave, avrebbero, molto probabilmente, prodotto una catastrofe: l'investimento dello schooner.

La piccola nave, di cui qui si parla, aveva nome Sofia, quello della figlia primogenita dell'Armatore, e che fu poi imposto dal Capitano all'atollo. Essa nave contava allora un anno di campagna, durante il quale aveva visitato le Indie, l'Australia e l'America meridionale. Partita dall' Inghilterra, nel 1852, approdó a Genova nel 1857, reduce da un viaggio di circumnavigazione, il primo stato compiuto da una nave coperta dalla bandiera, che fu poi quella d'Italia.

\section{Gruppo di Gilbert o di Kingsmill.}

L'isola Aroroe ha una estensione di 3 a 4 miglia con un piccolo lagone interno. È abitata da pochi indig'eni, che vivono ancora sotto l'impressione delle rapine degli schiavisti.

Tamana, ove crescono in abbondanza il cocco ed il pandano, non ha che 2 o 3 miglia di lunghezza. Dà buonissima acqua facendo pozzi nel suo centro.

Onatoa è un atollo abitato.

L'isola Peru è lunga parechie miglia, ma è molto stretta poichè varia in larghezza da $1 / 2$ miglio soltanto ad un miglio e poco più. Vien formata da una successione di collinette di sabbia, di corallo in frantumi e di conchiglie. Queste elevazioni sarebbero dovute, secondo alcuni viaggiatori degni di fede, alla violenza delle onde durante lo imperversare degli uragani. 
I prodotti dell' ora detta isola sono gli stessi delle altre del gruppo. Gli indigeni sembrano quivi dare maggior valore al pandano che all'albero del cocco. Si consuma nell' isola una grande quantità di frutti, con alcuni dei quali le donne preparano una certa qualità di pasta dolce che, disseccata, si rotola e si conserva.

\section{Gruppo Fenice.}

Comprende alcune piccole e basse isole corallifere - sette od otto - conosciute coi nomi di Swalow, Enderbury, Birney, Gardner o Kemin, Mc Kean, Hull e Sydney.

Il Commodoro Wilkes, che visitò il gruppo, dà i seguenti particolari intorno ad alcune:

L'isola Kemin o Gardner è molto bassa con un lagone poco profondo al centro, che non è navigabile.

L'isola Mc Kean è formata di coralli, sabbia e blocchi; è lunga $3 / 4$ di miglio e larga $1 / 2$ miglio.

L'isola Enderbury ha 3 miglia di lunghezza e 2 e $1 / 2$ di larghezza; è coperta parzialmente da meschina vegetazione.

L'isola Hull fornisce un po' d'acqua dolce ed ha alcuni alberi di cocco.

Queste che abbiamo descritte, tranne la Nuova Caledonia, sono le isole della Polinesia, che si trovano nell'orbita di attrazione di Tahiti e delle Figi.

La Nuova Caledonia potrebbe, per la sua estensione, per le condizioni del suolo e per la sua ubicazione esercitare essa pure ragguardevole influenza, ma il suo carattere di colonia penitenziaria togliendole ogni energia morale e politica, ne ha ristretto troppo la sfera di azione economica.

Tuttavia, l'Inghilterra diffidò sempre anche di questa circoscritta azione, per modo che, nella lotta di preponderanza impegnatasi fra lei e la Francia, nel Pacifico, il suo sguardo non si è mai rivolto meno verso la colonia caledoniana, di quello che si rivolgesse verso Tahiti. 
Per combattere intanto ogni influsso francese, da qualunque lato potesse sorgere, nello installarsi nelle Figi, l'Inghilterra conferi al suo rappresentante in quella regione ${ }^{1}$ estesi poteri giurisdizionali sopra tutte le isole del Pacifico occidentale non ancora occupate da nazioni europee.

Ma di codesto provvedimento la Francia non tenne alcun conto, e, nel 1883, tentó occupare il gruppo delle Nuove Ebridi compreso nella zona di giurisdizione britannica, ed aggregato molti anni prima, nominalmente, alla colonia inglese della Nuova Zelanda.

Sorsero allora vive rimostranze da parte del Gabinetto di Londra, ed una reazione violenta da parte di tutti i Governi australiani, i quali si riunirono, occasionalmente, in federazione per opporsi, a tutt'uomo, contro l' invasione francese, giungendo al punto di prendere formale possesso, in odio alla Francia, della costa della Nuova Guinea giacente di fronte alle Nuove Ebridi ed alla Nuova Caledonia. ${ }^{2}$

Il conflitto non tardò a cessare: la Francia fece rinunzia delle sue pretese di occupazione sulle. Nuove Ebridi, e l'Inghilterra sconfessò l'operato delle sue colonie australiane.

1 Il rappresentante della Corona inglese alle Figi, come abbiamo veduto altrove, porta il titolo di Alto Commissario del Pacifico occidentale e di Console generale per il Pacifico occidentale.

2 Proclamazione letta a Porto Moresby il 4 aprile 1883:

«Io Henry Majoribanros Chester, Magistrato residente all' isola Thursday, nella colonia Queensland, funzionante in dipendenza delle istruzioni ricevute dal Governo di detta colonia, prendo possesso di tutta quella parte della Nuova Guinea e isole adiacenti, che è compresa tra il $141^{\circ} \mathrm{e}$ il $155^{\circ}$ meridiano Est di longitudine, in nome della Regina, discendenti e successori. Nella presa di possesso ho alzata e salutata la bandiera inglese a Porto Moresby, nella Nuova Guinea, questo di 4 aprile, anno del Signore mille ottocento ottantatre ».

Lista dei luoghi della Nuova Guinea appartenenti all'occupazione, con gli abitanti di ogni villaggio e distretto:

Tale occupazione abbraccia 60 miglia di costa da Boera a Kerepunu, ossia 60 miglia per 40 dalla catena di Owen Stanley, e sono: Mouri, 100 abitanti; Munakahila, 300; Eikiri, 200; Kupele, 600; Logeri, 600; Faveli, 400; Moroka, 400; Maiara, 200; Jovi, 300; Epakari, 500; Epara, 300; Taburi, 400; Makapili, 400; Tubuselei (interno), 400; Kaile (interno), 1000; Kapakapa (interno), 1000; Palanai, 500; Boera, 300; Ponebada, 200; Port Moresby, 800; Vapukeri, 200; Pari, 300; Tupuselei, 350; Kaile, 350; Kapakapa, 300; Hula, 600; Papaka, 400; Kalo, 1500; Kerepunu, 1500; Kuaipo, 600 ; Animarupa, 300 ; Koilapuans (sparsi), 1000. Totale 16,500 abitanti corrispondenti a circa 7 persone per miglio quadrato. 
Ogggi che non è più questione, per alcuno, dell' accennato gruppo d'isole, una lunga distesa del litorale della Nuova Guinea è caduta in mano dell' Inghilterra, la quale lascia alla Germania il possesso di quella porzione del vasto territorio papuasiaco, che libera dalla sovranità britannica, puó per avventura convenirle.

Il motivo principale, se non l'unico, degli sforzi che Francia ed Inghilterra spiegano nell'Oceania, per attrarre a sè il maggior numero possibile d'isole, vuolsi rintracciare nel bisogno, fortemente sentito, d'importare dalle. località povere nei luoghi ricchi, per produzione del suolo, lavoranti indigeni.

Gli abitanti in tutta o quasi tutta la Polinesia si trovano estremamente sparsi, mentre, qui come in ogni paese intertropicale, $\dot{e}$ soltanto ove la popolazione è densa che, in difetto di schiavi, gl' indigeni possono rendere proficua la coltura agricola. E non è che a costoro, cioè alla gente di colore, che si possa nell'Oceania affidare il lavoro del campo, poichè fallirono sempre tutti i tentativi fatti in varie parti del globo, in questi ultimi tre secoli, per introdurre agricoltori bianchi in regioni tropicali, e fallirono perfino nelle località montuose dotate di clima temperato. ${ }^{1}$

\section{ISOLE DELL'OCEANIA}

CHE SI TROVANO FUORI DELLA SFERA D'INFLUENZA POLITICO-ECONOMICA

DI TAHITI E DELLE FIGI.

Isole di Sandwich o Hawaiane.

Queste isole, che formano il regno di Hawaii, costituiscono una ricca, bella e interessante catena; sono in numero di otto, inclusevi una o due isolette. Tale catena corre da S. E. a N. O., giacente in mezzo dell'Oceano Pacifico, nella latitudine di $19^{\circ} 22^{\prime} \mathrm{N}$. e nella longitudine $155^{\circ} 16^{\prime} \mathrm{W}$.

La superficie totale del gruppo è di 6000 miglia quadrate così ripartite: Hawaii (anteriormente Owhyee), 4000; Maui, 620; Oahu, 530; Kaui, 500; Mo-

1 Vedasi Acclimatazione della specie umana, pag. 267. 
lokai, 167; Lanai, 100; Niihau, circa 70; e Kakoolaui, circa 60. Il censimento ufficiale del 1866 dava una popolazione di 69,959 anime, di cui 4194 stranieri (compresi i chinesi) e 59,765 indigeni.

Le importazioni ascesero, nel 18 1 , a lire sterline 325,176 , e le esportazioni a lire sterline 378,413 . Il prodotto principale è rappresentato dallo zucchero.

Il naviglio hawaiiano consta di 8,068 tonnellate, delle quali 414 a vapore. Nel porto di Honolulu (capitale del gruppo) approdarono, nel 18\%1, 163 bastimenti mercantili di varie nazioni, del tonnellaggio complessivo di 102,1 2 tonnellate.

La forma del Governo è monarchica costituzionale: un Re, un'Assemblea di nobili ed una Camera di rappresentanti del popolo. L'indipendenza del regno di Sandwich fu formalmente riconosciuta, nel 1843, dai Governi di Francia e d'Inghilterra.

Il nostro egregio Comandante Napoleone Canevaro, che, nel 1878, in un viaggio di circumnavigazione, al comando del regio incrociatore Cristoforo Colombo, visitò le isole di Sandwich, ci fornisce intorno ad esse le seguenti interessanti notizie: ${ }^{1}$

Le isole Sandwich hanno un bell'avvenire per la loro posizione eccezionalmente favorevole, essendo certo che col tempo vi si incrocieranno le linee di navigazione a vapore, che traverseranno il Pacifico col commercio asiaticoamericano, mentre già al presente vi passa una linea importante per l'Australia e la California, e sta per passarne un'altra fra il Perù e la China. Oltre a ciò, le varie isole abbondano di terreni fertilissimi per prodotti tropicali e per molti prodotti de' paesi temperati; attualmente, le coltivazioni principali sono quelle dello zucchero, caffè, cotone ed olio di cocco. Però la popolazione indigena va diminuendo in modo sensibile ed è pure infingarda, sicchè mancano molto le braccia, ed il Governo attuale si è preoccupato della cosa al punto di cercare coloni da ogni parte, e dovrà finire per accettare l'emigrazione di coolies chinesi, che, sino ad ora, ha cercato di scartare per poca simpatia e molto timore della razza del Celeste Impero.

La colonia bianca è di circa 3000 persone in massima parte americani, tedeschi ed inglesi, sotto le quali bandiere è anche fatto il commercio. È da notare tuttavia che la marina mercantile avaiana ha aumentato sensibilmente, e che molti armatori forestieri stabiliti nell'arcipelago ne preferiscono spesso

1 Rivista marittima, Anno XI. 
la bandiera, la quale gode presso i paesi civili de' vantag'gi accordati a quelli delle grandi nazioni, senza incorrere ne" rischi di guerra.

Politicamente, le nazioni che hanno maggiore influenza sopra questo piccolo regno isolano sono gli Stati Uniti, l'Ingliilterra e la Francia, e tutte e tre tengono un ministro residente accreditato presso il Governo a Honolulù; ma, in questo momento, l'influenza americana sorpassa di gran lunga le altre, sia per il numero degli americani stabiliti in paese, sia per la vicinanza della costa di California, e sia per i vantaggi destramente accordati dalle dogane degli Stati Uniti alle merci di provenienza avaiana.

Di bastimenti italiani non ne approda nessuno da parecchi anni.

\section{ISOLE CAROLINe.}

La loro superficie è di miglia geografiche quadrate 46.1. Popolazione: abi$\tan$ ti 20,000 .

\section{IsOLE PeLEW.}

¿̀ un gruppo d'isole situato a 450 miglia a levante delle Filippine, ed all'estremità occidentale delle Caroline. Include circa 20 isole, che formano una catena stendentesi circa 120 miglia da S.S. O. a N. N. E.

L'isola maggiore è Babelthouap, lunga 28 miglia e larga 14. Veduto il gruppo dal largo mare appare montuoso ed accidentato; ma il suolo è ricco e fertile, e l'acqua vi abbonda. Crescono colà l'albero del pane, del cocco, la banana, la canna da zucchero, i limoni, gli aranci ed ogni altro albero o frutto tropicale. Gli abitanti ascendono a circa 10,000; sono di razza malese. Operosi agricoltori, si manifestano molto primitivi nell'arte di costruire canoe. e, specialmente, negl' indumenti. Gli uomini vanno nudi, e le donne poco meno. Nel 1783 l'Antelope naufragò nelle isole Pelew, ove l'equipaggio fu raccolto dagli indigeni con molta bontà. Più tardi però queste buone disposizioni verso i bianchi mutarono, giacchè alcuni bastimenti, che visitarono le dette isole, corsero pericolo di essere saccheggiati.

\section{Isole Marianne o de' Ladroni.}

È un gruppo di circa 20 isole montuose, ricche di vegetazione e d'acqua, e la cui superficie complessiva ascende a 1254 miglia quadrate. Produce l'albero del pane, il cocco, la banana, il riso, il grantureo, il cotone e l'indaco. Gli 
animali domestici vi sono adesso comuni. Allorchè lo si scoperse, la popolazione fu stimata ascendere a 100,000 anime; adesso essa si trova ridotta a circa 5500 abitanti. Gl' indigeni sono docili, religiosi, amabili ed ospitalieri, rassomigliando nel tipo a quelli delle Filippine. Sotto il punto di vista commerciale le isole de' Ladroni sono molto importanti per gli spagnuoli. L' isola maggiore è Guayan, che ha 90 miglia di circonferenza; in essa si trova la capitale - Sant'Ignazio de Agramea - che è la sede del Governo spagnuolo.

\section{Arcipelago delle Galapagos. ${ }^{1}$}

All'Ovest delle coste dell'Equatore, e ad una distanza di 9 gradi, sorge l'arcipelago delle Galapagos formato da tredici isole e da numerosi isolotti.

Interessanti per la loro posizione geografica, grazie a cui dovranno necessariamente diventare lo scalo di provvigionamento dei piroscafi percorrenti le grandi linee di navigazione lungo la costiera occidentale americana, le Galapagos sono poi interessantissime per la loro costituzione geologica, la loro fauna, la loro flora.

Di formazione vulcanica tutte quante, esse vanno soggette a condizioni fisiche e climatologiche singolarissime, le quali vi determinano due spiccate zone o regioni; l'una bassa, calda, secca, sterile; l'altra alta, temperata, umida, fertile: da ciò l'aspetto curiosissimo che, nel loro insieme, presentano le isole da lontano. Sopra una base, che si eleva quasi 200 metri sul pelo del máre e la quale ha l'apparenza di una massa vitrea, chiazzata di larghe macchie, si erge con repentino distacco un monte di forma irregolare, la cui ricca, lussureggiante vegetazione apparisce solcata da lunghe e larghe striscie nere, le quali partendo dalla vetta del monte, come raggi da un centro, scendono senza interruzione fino al mare, conservando inalterato, lungo tutto il percorso, il loro carattere. Queste striscie formate da correnti di lava, sono il migliore e più sicuro indizio che in talune isole, come ad esempio nell'Albermale e nella Narboroug, i vulcani hanno dato indizio di attività fino ai tempi recenti.

Nella regione bassa, stendentesi dal pelo del mare ad una altezza di poco superiore ai 200 metri, non piove mai : il suolo pertanto ha conservato quasi per intiero l'originario suo aspetto; ivi sono roccie intatte, campi estesi di lava or nera, or bigia, or rossiccia, con la superficie vitrea, scoreiforme, impraticabile; ivi una scarsa e rachitica vegetazione.

1 Vedi carta annessa. Dobbiamo alla cortesia dell'esimio naturalista Dr. T. Wolf i particolari concernenti la fisonomia fisica delle Galapagos, ed a quella della famiglia Villamil i cenni storici. 
Nella regione alta, dove, nell' inverno, da febbraio a maggio, le pioggie sono abbondanti e frequenti, dove alle grandi pioggie succedono le copiose rugiade, il suolo ha perduto le remote apparenze e si è completamente trasformato. Le rocce dure e compatte, sotto la doppia azione chimica e meccanica della pioggia e del sole, si decomposero e formarono un grosso strato di terra argillosa che, commista coi detriti vegetali, produsse ridenti praterie ed una vegetazione svariata e robusta.

Nel loro insieme le Galapagos occupano uno spazio di 6000 e più miglia

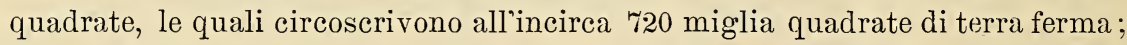
disgraziatamente, però, questa è compresa, nella massima sua estensione, nella zona bassa e sterile anzichè nella alta e fertile.

Fra le isole che, non superando in altezza 200 metri, appartengono esclusivamente alla regione sterile, alcune, come le Hood, Barrington, Chatam nella sua parte settentrionale, sono piuttosto ampie; di qualche estensione, quantunque in proporzioni sempre minori, sono pure le isole Floriana, Indefatigable, Albemarle, Chatam nella parte meridionale, le quali appartenendo alla regione alta, sono per conseguenza propizie all'agricoltura.

Affatto disabitate in origine, esiste oggi in parecchie di esse, e specialmente nella Floriana e nella Chatam meridionale, buon numero di animali, come cani, capre, maiali, bestie bovine e cavalline, stativi importati: tutti questi animali trovano un abbondante ed ottimo nutrimento nei pascoli naturali, che composti di gramigne ed altre ottime erbe, richiamano alla memoria i grandi ed ubertosi prati dell'Europa centrale.

I detti animali, non avendo nemici fra quelli indigeni, si sono propagati con sufficiente rapidità; chè se, in ogggi, sono meno abbondanti i prodotti caprini e bovini, ciò dipende dallo straordinario numero di cani, i quali, diventati selvatici, fanno una caccia feroce alle capre ed ai vitellini. Nelle isole, solo i rettili sono indigeni, e di questi ve n'è grandissima copia; tutti però inoffensivi, non essendosi mai rintracciata specie velenosa, neppure fra le quattro o cinque differenti classi di colubri; abbondano poi le tartarughe di specie grande.

Malgrado le grandi pioggie e le abbondanti rugiade, che cadono nella regione elevata, pochi sono i serbatoi di acqua dolce, opponendosi alla loro formazione il sottosuolo vulcanico; le sole isole Chatam e Floriana fanno in questo una eccezione, essendo abbastanza ben provviste di acqua, ed è certamente a questa causa che si deve la notevole differenza, che si nota fra la quantiti di bestiame esistente nelle dette due isole, e la scarsa quantità, che si trova nelle altre.

A Chatam, alla Floriana, le piccole lagune, le lievi conche, i pozzi naturali sparsi in ogni dove, si riempiono durante l'inverno, e provvedono di poi, 
nella stagione estiva, ai bisogni del bestiame, a cui non manca mai l'acqua per bere; i piccoli pozzi, sia per il consumo dellacqua che vi fa il bestiame, sia per la naturale evaporizzazione, finiscono per vuotarsi e rimanere asciutti, ma per breve tempa e senza che il bestiame ne risenta danno, giacchè l'acqua necessaria per bere la si trova sempre in alcuni pozzi inesauribili, due dei quali, per esempio, esistono nell' isola Floriana, malgrado che, in questa, gli altri serbatoi naturali si prosciughino più frequentemente e prima di quelli dellè altre isole, per essere il terreno coltivabile e produttivo meno elevato.

Salubre dappertutto, il clima delle Galapagos ha le tre gradazioni di caldo, temperato e freddo: alla spiaggia la temperatura dell'aria è piuttosto bassa, stando sempre di un grado circa al disotto della temperatura del mare; questo fenomeno è dovuto alla vasta superficie acquea, in mezzo alla quale sorgono le isole, ed alla grande corrente fredda di Humbold, che le bagna tutto intorno, non bastando a modificare questo costante dislivello termometrico i venti dominanti, che soffiano dal S. E.

La flora delle Galapagos, suscettibile, grazie alle diversità del clima, delle più svariate produzioni, comprese tra la banana dei climi caldi e la mela dei climi freddi, ha in generale l'impronta del tipo americano, tanto rispetto alla affinità botanica dei generi e delle specie, quanto rispetto al suo abito esterno; mancano però alla flora delle isole quella vivacità di colori, quella bellezza di forme particolari, per le quali è così meritevole di speciale attenzione la flora sud-americana.

In questa, la magnificenza delle foreste consiste, per molta parte, nell'artistico e lussureggiante fogliame dei monocotiledoni, delle palme, musacee, zingiberacee, aroidee, ecc., famiglie tutte che mancano alle Galapagos, ove mancano del pari quasi totalmente le epifiti che, ornamento speciale delle foreste equatoriali, non sono rappresentate nell'arcipelago che da due bromeliacee e due orchidee insignificanti. I caratteri particolari, adunque, che, a prima vista, emergono nella flora delle Galapagos e la differenziano da quella del continente sono: la piccolezza degli organi fogliacei, la mancanza di bei fiori, la scarsità di epifiti e parassiti, l'assenza di liane e rampicanti.

È fuor d'ogni dubbio che i deserti del continente offrono un maggior numero di forme tropicali, ma ciò non si può attribuire alle sole condizioni del clima, tanto più se si ponga mente che il maggior numero delle piante fanerogame sono endemiche all'arcipelago e non si trovano in altre parti: ecco un altro dei misteri della natura che alla scienza è riservato di spiegare!

Del resto alla stranezza della natura organica risponde con perfetta armonia la natura inorganica, la quale si rivela sotto aspetti veramente fantastici.

- Premesso che, nelle Galapagos, a differenza di quanto una volta si supponeva, non esiste alcun minerale da far valere, non esiste guano, non fosfato 
di calce, la loro natura inorganica si presenta sotto le seguenti forme: centinaia di fucine ciclopiche coi vasti forni spenti e con immani massi di nerissima angolosa lava sovrapposti arditamente gli uni sugli altri; avanzi di rocee bruciate, fra le quali è prigioniero il tronco corpulento di una spina alba (Cereus), oppure d'un fico d'India (Oputia Galapagos), o sulle quali muove, con mirabile flemma, le deformi sue membra la Testudo elephantopus, onde -salire per la trigesima volta sopra uno scoglio, dal quale precipitò per ventinove volte; gruppi di brutte e strane iguane marine (Amblymynchus cristatus) che colla bocca spalancata e le membra distese si godono il sole. Veri paesaggi antidiluviani, quali i geologi sono avvezzi dipingere nelle loro descrizioni dei fossili.

Dato cosi un cenno sommario delle condizioni geologiche, della fauna e della flora delle isole, ne diremo in brevi parole le vicende politiche da una cinquantina d'anni in poi.

L'arcipelago delle Galapagos, benchè noto ai naviganti e ai greografi, non fu mai oggetto di mire speciali da parte di qualcuno, insino all'anno 1832, nel quale furono le isole occupate, in nome della Repubblica dell'Equatore, dal Colonnello Josè Joaquin Olmedo, che precisamente il 12 febbraio del detto anno ne prese possesso.

In onore del Generale Florés, allora Presidente della Repubblica, l'isola San Carlo, dove si celebrò la cerimonia della presa di possesso e che venne designata quale capitale dell'arcipelago, ricevette il nome di Floriana, che conserva ancora oggidi.

A indurre il Governo equatoriale alla impresa suacennata ebbe gran parte il Generale Villamil, titolato dell'Equatore, ma nativo della Nuova Orleans. Uomo di elevato carattere, di molta energia, di larghe vedute, egli sosteneva, nel novembre 1831, la convenienza per la Repubblica di avocare a sè la sovranità territoriale dell'arcipelago, e nello stesso mentre chiedeva al Governo ed otteneva, per conto di una Società colonizzatrice da lui organizzata, la proprietà dei terreni di una delle isole, a sua scelta; scelta che cadde poi sull' isola Floriana.

Il Villamil, avuta la concessione della Floriana, vi fondò una fattoria agricola, di cui furono elementi costitutivi ottanta soldati del battaglione Florés, i quali essendosi ribellati, erano stati condannati alla decimazione: il Villamil perorò presso il Governo la causa di cotesti disgraziati, ed ottenutane la commutazione di pena, li prese con sè, li trasportò alla Floriana, ripartì fra loro le terre da coltivare, somministrando ad essi utensili, semi, animali e quant'altrọ occorreva all' impianto dell'azienda agricola, e provvedendo inoltre al loro 
sostentamento, fintantochè non furono in caso di provvedervi loro stessi colle risorse del suolo.

Avuto nel 1832 il governo dell' arcipelago, nella sua doppia qualità di rappresentante del Governo in quelle isole e di rappresentante della Società colonizzatrice, egli si dedicò con grande impegno all'avvenire della nascente colonia. Con ingenti spese, con gravissime fatiche Villamil importò dal continente, nella Floriana, una quantità notevole di animali domestici, i quali, avendo trovate molto propizie le condizioni per propagarsi, aumentarono in modo che si potè poco di poi prelevarne diverse coppie e distribuirle nelle altre isole, a Chatam segnatamente, ove figliarono in modo straordinario.

Il Generale Villamil, sebbene fosse quasi al principio dell'impresa, abbandonato non si sa perchè dai soci, non desistette dall' impresa, ma da solo si assunse il còmpito di mantenere gli impegni contratti verso lo Stato.

La fama della prosperità e del benessere, che si godeva alle Galapagos, non tardò a propagarsi, ed il solerte Villamil ebbe il conforto di vedere accorrere alle isole speculatori per tentarvi la fortuna.

Sotto la saggia e previdente di lui amministrazione, durata dal 1832 al 183\%, la colonia andò sempre crescendo prospera e fiorente: tale era il rispetto, la riverenza che a tutti inspirava il Villamil, tale il timore che incuteva ai cattivi, che la colonia, finchè vi rimase egli al governo, non risentì alcun danno da un improvvido decreto del Generale Florés, che nel marzo 1833 destinava la Floriana a luogo di deportazione, per scontarvi la pena dell'esilio. Il Governo equatoriale, dando al decreto del Generale Florés un'applicazione, che non poteva e non doveva avere, si valse delle Galapagos per diminuire la popolazione delle sue carceri criminali, e vi fece trasportare tutti i peggiori delinquenti, senza menomamente preoccuparsi del modo di provvedera al loro ricovero, alla loro custodia, al loro sostentamento.

Per la nascente colonia, la triste popolazione inviatavi dal Governo equatoriale fu un pessimo acquisto; pure, mercè la ferma, oculata e provvida amministrazione del Villamil, la colonia non si arrestò per questo nel suc prospero sviluppo. Disgraziatamente, nel 183\%, il Villamil veniva chiamato ad altri uffici e doveva cessare dalle sue funzioni di Governatore dell'arcipelago; succedevagli il Colonnello Williams, che mancando alla fiducia in lui riposta. commise, nell'esercizio delle sue delicate attribuzioni, ogni sorta di eccessi, violenze, estorsioni, crudeltà inaudite. Sotto l'amministrazione deplorevole del Williams, tutti quei torbidi elementi, che il Governo equatoriale aveva. tolti dal carcere per inviarli alla Floriana, ebbero il sopravvento e non andò guari che la colonia si demoralizzò completamente; i migliori dei suoi membri, invasi da paura, fuggirono, ed il numero dei coloni si ridusse, in poco tempo, ad un quarto di quello che era precedentemente. 
Il Williams stette alla Floriana dal 1837 al 1841, ma bastarono questi cinque anni per rovinare completamente ogni cosa; epperò, allorquando, nel 1842, l'ottimo Generale Villamil fece ritorno alla Floriana, la colonia era quasi del tutto scomparsa, l'arcipelago essendosi trasformato in un covo di briganti e di pirati, i quali commettevano impunemente atroci delitti.

Lunga purtroppo ad enumerare sarebbene la serie; basti per tutti accennare al barbaro assassinio consumato appunto verso quell'epoca sulla persona del Capitano Copello e di uno dei suoi figli. Comandante il padre di una nave mercantile sarda, di cui era secondo il figlio, furono essi obbligati dall'equipaggio, ammutinatosi, ad approdare alle Galapagos, dove padre e figlio trovarono orrida morte per mano de' coloni, che predarono la nave.

Intolleranti di qualsiasi legge o freno, i briganti e predoni, che avevano preso stanza nell'arcipelago, commisero per dieci anni ogni specie di misfatti godendo della più larga impunità. Nel 1852, cercava rifugio a Chatam il Generale Mena, statovi esiliato dal Governo equatoriale: il Generale installavasi nell' isola e stringeva relazione con cinque coloni, i quali rappresentavano la parte meno triste della popolazione; ebbene, una notte il Generale ed i cinque suoi amici furono trucidati, andando a sacco le rispettive loro case.

Questo fatto parve destare il Governo equatoriale dall'abbandono in cui, per 20 anni, aveva lasciato l'arcipelago; convocata infatti l'Assemblea nazionale in Guayaquil, questa deliberava che si dovesse ritenere legittimo ed inconcusso il diritto vantato sui terreni della Floriana dalla Società colonizzatrice (la quale però aveva cessato di esistere) e dal Generale Villamil, consocio principale; che allo Stato dovesse riservarsi la facoltà di disporre di quei terreni, che si riconoscessero di utilità pubblica; che nè l' isola nè i suoi abitanti potessero mai emanciparsi dall'impero è dominio eminente, che appartenevano esclusivamente alla nazione.

Non valsero tuttavia questi provvedimenti di natura legislativa, perchè non suffragati mai da altri efficaci nel campo esecutivo, a ridare la primitiva prosperità alla colonia dell'arcipelago; nella stessa isola Floriana, malgrado le cure del Villamil, rimasero sempre depresse le sorti e le condizioni dei coloni.

Non senza che ad affrettarne la fine contribuissero i disinganni ed i dispiaceri provati, per la mala riescita dell'impresa da lui tentata, nel 1832, alla Floriana, con tanto prosperi inizi, il Generale Villamil moriva a Guayaquil, nel 1866.

Morto il Generale, le cose alle Galapagos volsero più che mai a precipizio, non curandosi le autorità equatoriali di ristabilirvi l'ordine, essendone impotenti gli eredi del Villamil.

Per tre anni, le isole dell' arcipelago furono libero campo alle imprese di 
avventurieri, i quali vi approdavano col pretesto di intraprendervi speculazioni, ma in sostanza per appropriarsi quel poco che in esse rimaneva, che esse ancora producevano. Gli eredi del Generale non cessavano dallo insistere presso il Governo per ottenerne aiuto e protezione, non fu però che nel 1869, dopochè eransi aggravate più che mai le condizioni delle isole, che la signora Ana Villamil, figlia primogenita, potè ottenere l'intervento del Governo equatoriale contro le imprese degli usurpatori delle isole, e specialmente contro le mene di certo Dottor Benigno Malo, il quale, sconoscendo i diritti della famiglia Villamil, e mirando a sostituirsi a questa, nella proprietà della Floriana, tentava di ottenere, in affitto, le isole dell'arcipelago, direttamente, dalle autorità governative di Quito.

La signora Ana Villamil, invocando la deliberazione dell'Assemblea nazionale del 26 settembre 1832, la quale aveva in modo solenne riconosciuto i legittimi e inconcussi diritti del Generale Villamil sui terreni della Floriana, chiedeva al potere esecutivo della Repubblica che respingesse le proposte del Dottor Malo, e tutelasse gli eredi contro le usurpazioni delle quali erano vittima da tre anni. Il Governo equatoriale faceva ragione alla giusta richiesta, e mentre dichiarava al detto Dottore che erano inaccettabili le sue proposte, perchè non appartenente allo Stato la proprietà della Floriana, ordinava al Governatore della provincia del Guayas (Guayaquil) di provvedere, affinchè i beni di proprietà degli eredi Villamil non soffrissero pregiudizi da parte degli intraprenditori, che facevano lavorare nell' arcipelago delle Galapagos.

Posto, in tal modo, un lieve freno alle continue ruberie di coloro che approdavano alle Galapagos, fu possibile agli eredi non molto di poi, cioè nel 18\%, dare in affitto le loro proprietà al signor Josè Valdizan, che le prese esclusivamente per la raccolta dell'orcella, lichene del genere Rocella, che vive esposta ai venti di mare sulle rocce e gli arbusti, e dal quale in Europa si ritrae una buona tinta rossiccia.

Esaurita in un paio d'anni l'orcella, il Valdizan lasciò le Galapagos per intraprendere un viaggio in California; ma ritornatone tre o quattro anni di poi, volle ristabilirsi nell' arcipelago, onde riattivarne la coltivazione coi criterî e coi modi, con tanta buona fortuna, usati dal Villamil dal 1832 al $183 \%$.

Accordatosi cogli eredi del Generale, circa il canone annuo ad essi dovuto, per l'affitto delle terre e per la cessione, in usufrutto, del bestiame, nelle isole esistente, il Valdizan fissò la propria residenza alla Floriana, che diventò il centro delle sue nuove operazioni.

Aveva egli condotto seco molti braccianti arruolati in vari luoghi, e, da quelli secondato, aveva iniziato, con abbastanza prospera fortuna, la sua intrapresa; senonchè, l'errore, in seguito commesso, di accettare tra i suoi dipen- 
denti, degli evasi e liberati dai luoghi di pena di Guayaquil, doveva non molto dopo riescirgli fatale.

In un'isola priva di qualsiasi autorità governativa, priva di qualsiasi forza a tutela dell'ordine, i tristi elementi dati dalle carceri di Guayaquil non tardarono a produrre disordini, ribellioni, attentati criminosi di ogni natura: il Valdizan, coadiuvato da pochi coloni di fiducia, si era sforzato invano di richiamare al dovere i facinorosi, di rimettere l'ordine nella sua numerosa famiglia: i tentativi di repressione da lui fatti non valsero che ad inasprire maggiormente i malvagi, che, unitisi in congiura, ne decisero l'uccîsione.

La mattina del 23 luglio 18\%, infatti, quattro braccianti, fra i peggiori, presentavansi improvvisamente nella casa del Valdizan, e sorpresolo con inganno, gli vibrarono un colpo di pugnale al ventre ferendolo mortalmente: senza perdersi d'animo egli lottò fino a che potè, ma sentendosi venir meno le forze, per la grande perdita del sangue, nè volendo cadere sotto i colpi dei suoi assassini, ebbe ancora tanta energia per buttarsi giù da una finestra chiedendo aiuto: accorse per la prima alle sue grida la moglie, che appena fu in tempo a raccoglierne gli ultimi sospiri. Propagatasi intanto la notizia del triste accaduto, furono solleciti ad accorrere sul luogo i coloni di fiducia, i quali armatisi alla meglio coi ferri e gli strumenti capitati loro sotto mano, si buttarono sugli assassini, e, non curando le ben nutrite fucilate che a loro difesa questi sparavano, li uccisero tutti, ad eccezione di uno, che, nel fitto della mischia, riescì a fuggire.

Morto il Valdizan, le isole Galapagos ricaddero nel primitivo abbandono: partirono tutti quanti i coloni dalla Floriana, e più non rimase che una quarantina di famiglie nell' isola Chatam, le quali, sotto la dipendenza di certo Manuel Coboz, attendono ancora in oggi all'allevamento del bestiame ed al raccolto dell'orcella: il Coboz paga un annuo tributo alla famiglia Villamil.

Parve un momento, verso il 18\%9, che l'arcipelago delle Galapagos dovesse diventare il centro di importanti intraprese agricole ed industriali: circa quel tempo, infatti, una Società peruviana, per mezzo di rappresentanti inviati a Guayaquil, fece alla famiglia Villamil proposte di cessione dei suoi diritti sulle isole dell'arcipelago, offrendo come compenso la somma di 50,000 pezzi d'argento, corrispondenti a 210,000 lire italiane.

Gli eredi Villamil rifiutarono quelle proposte, ed i rappresentanti della Società si rivolsero allora al Governo equatoriale chiedendo il privilegio, dietro congruo canone, di estrarre dalle Galapagos guano ed ogni altro qualsiasi prodotto industriale del suolo.

Il Governo acconsentì; poco di poi comparve nelle acque dell'arcipelago una nave della Societa, e vi si trattenne alcuni mesi. Quale sia stata la 
missione da quella nave compiuta, lo si è sempre ignorato; il fatto certo è, che, dalla sua partenza in poi, nessuno intese più parlare di essa, nè dei suoi progetti.

Dalle cose fin qui esposte appare quali siano le condizioni politiche, fisiche, geologiche delle Galapagos, quali siano i caratteri generali della loro flora e fauna.

Molto ristretta la flora, giova però avvertire che la salubrità del clima, la notevole varietà di temperatura, che in una plagra di terreno ristretta va dall'uno all'altro estremo del caldo e del freddo delle zone temperate, fanno con buon fondamento presupporre, nelle Galapagos, una speciale attitudine alla coltivazione di molti prodotti propri dei paesi caldi, temperati e freddi. Non dovrebbe dunque essere difficile, con criteri razionali di coltivazione, rendere abbastanza ricca e svariata la flora dell'arcipelago.

Vicino alla canna da zucchero ed alla banana, potrebbero benissimo crescere la patata e qualsivoglia altra specie di legumi; potrebbero prosperare alberi fruttiferi di svariato genere e forse anche la vite; e se più ampia fosse la parte elevata delle isole, quella cioè propizia all'agricoltura, non vi ha dubbio che l'avvenire agricolo delle Galapagos potrebbe da solo consigliare l'occupazione delle isole; disgraziatamente, sopra.le 720 miglia quadrate circa di terra, comprese nell' arcipelago, appena 60 sono suscettibili di esser coltivate; troppo limitata estensione perchè delle Galapagos si possa pensare a fare una importante colonia di esportazione di prodotti agricoli.

Della produttività agricola delle isole, sebbene per se stessa insufficiente ad alimentare una colonia, va però tenuto molto conto da chi, ritenute le condizioni nautiche delle Galapagos, considerata la loro fortunata situazione nel Pacifico, le studiasse dal punto di vista della importanza che acquisteranno il giorno in cui sarà aperto alle navi il canale di Panamà.

E poichè a noi sembra che Nazione e Governo abbiano ugualmente l'obbligo di avvisare fin d'ora ai mezzi più efficaci, onde trarre per l'Italia tutto il magrgiore possibile utile dalla grande nuova strada, che si andrà ad aprire, così è che non ci sembra nè disutile, nè fuor di proposito sottoporre all'apprezzamento del lettore talune considerazioni sulla somma utilità che l'Italia si assicuri fin d'ora un punto di approdo e di rifornimento per le navi destinate a spingersi, attraverso il canale di Panamà, dall'Atlantico nel grande Oceano.

La corrente fredda di Humbold lambe il gruppo delle Galapagos seguendo la direzione di N.O. con una forza crescente nei canali del gruppo, fino a raggiungere alcuna volta la velocità di 3 miglia l'ora. Questa corrente, note- 
volissima nei dintorni dell'arcipelago, è di grave ostacolo alla navigazione ì vela, ma non offre alcuna difficoltà alla navigazione a vapore.

L'elevazione delle isole e la costante visibilità della loro base rendono facili gli approdi, mentre i fondi, che sono comunemente costituiti da sabbia fina, corallo e conchiglie spezzate, presentano buoni ancoraggi; i migliori sono quelli delle isole Chatam, Floriana, Albermale e Santiago, ove i fondi variano vicino a terra dai 10 ai 30 metri.

Primeggia su tutti, per sicurezza e facilità di accesso, l'ancoraggio dell'isola Floriana, detto Post Office Bay, perchè già un tempo stazione delle navi baleniere, le quali lo avevano scelto per punto di ritrovo, onde scambiarsi vicendevolmente la corrispondenza; a Post Office Bay regna costantemente calma di mare e di corrente; si difetta però d'acqua dolce, che bisogna andar a caricare colle imbarcazioni a Black Beach Road. Inconveniente abbastanza grave, ma che potrebbe esser tolto con tutta facilità, a mezzo di apposita tubulatura, che, partendo dai pozzi e serbatoi naturali esistenti nella zona elevata dell'isola, giungesse sino alla marina di Post Office.

Nè mancherebbe l'acqua alle esigenze anche di un forte consumo, giacchè ad assicurarsene la quantità necessaria basterebbe allargare gli attuali serbatoi naturali, costruirne degli artificiali, e condurre negli uni e negli altri le abbondantissime acque delle piogge invernali.

Dotata di comodo ancoraggio, suscettibile di possedere, proprio alla marina, una sufficiente quantità di acqua dolce per le navi che lì approdassero, la Floriana merita una specialissima attenzione, per la sua favorevole situazione nel grande Oceano, in relazione all'avvenire che il taglio dell'istmo di Panamà serba al mare, che bagna le coste occidentali dell'America centrale.

Chi, trascurando il dettaglio, mirasse unicamente ai punti estremi della grande arteria commerciale a cui darà vita il nuovo canale, ed alle importanti diramazioni dell'arteria medesima, noterebbe subito, come soste indispensabili per le navi a vapore destinate a seguire i detti corsi, le isole Sandwich, l'arcipelago di Tuamotì o quello delle Marchesi, mentre gli sfuggirebbe forse, a prima vista, l'arcipelago delle Galapagos, situato a sole 900 miglia da Panamà, vale a dire ad una nona parte della distanza che separa, in media, i due estremi della mentovata grande arteria. Però, poco di poi, senza una ponderazione acuta, riconoscerebbe, come le Galapagos, e l' isola Floriana, specialmente, siano destinate a diventare uno scalo molto interessante per la navigazione.

L'arcipelago in parola trovasi, è verissimo, vicino all'istmo; ma se tengasi nota che in esso ha termine una zona assai estesa di calme, la quale fa capo a Panamà, non si indugierà ad ammettere che ogni piroscafo, attratto 
nei paraggi delle Galapagos dalla brevità del cammino, sentirà il bisogno di rifornirsi di combustibile e dovrà fare scalo in esse.

Nè saranno soltanto i piroscafi provenienti dall'Atlantico e diretti nel grande Oceano, i quali, attraversato il canale, proveranno il bisogno di fare scalo alle Galapagos, per rifornirsi di combustibile ed altro, ma altrettanto e più lo proveranno le navi dedite al commercio marittimo sulla costiera occidentale del continente americano; i piroscafi, a cagion d'esempio, che lasciato il Callao o Valparaiso, dirigeranno per S. Francisco di California, Acapulco, Unione, ecc., raramente potranno fare a meno di visitare le Galapagos, e, per conseguenza, gettare l'àncora alla Floriana, punto naturale d'intersecazione delle navi che dall'Atlantico, pel canale di Panamà, si recheranno nel grande Oceano.

La speculazione commerciale, pronta sempre ad impossessarsi di quelle risorse, di quei mezzi che le presentino sicurezza di guadagno, non indugierà a rivolgersi sul gruppo delle Galapagos, appena ne avrà avvertita l'importante e fortunata posizione rispetto al transito marittimo; e tra le nazioni più ricche di naviglio mercantile moderno, si stabilirà una gara vivace per assicurare a sè la proprietà dell'importantissimo approdo.

In questa gara inevitabile, necessaria, la vittoria rimarrà al più ricco, al meglio armato, al più forte, se chi lo è meno non sappia, con saggia previdenza, con ardita iniziativa, assicurarsi, fin d'ora, quei vantaggi, che non saranno più suoi, ma di altri il giorno in cui il fatto avrà dimostrata la somma utilità del possesso delle Galapagos.

Poche nazioni, per non dire nessuna, hanno nel Pacifico i vistosi notevolissimi interessi che vi ha l'Italia: gl'italiani adunque debbono accuratamente valutare ogni probabile effetto economico della futura grande via interoceanica, e provvedere in tempo ai casi loro.

Ovvio riesce, perchè palesi, indicare le felici conseguenze, che, per il nostro paese, deriveranno dal taglio dell'istmo di Panamà : si stabilirà un attivissimo va e vieni di emigrati italiani fra l'Italia, le due Americhe, l'Australia, la Nuova Zelanda; si farà viva ed incalzante la concorrenza del naviglio nazionale coi navigli stranier per il trasporto di passeggieri e merci dall' Europa e dalle Americhe alle località citate; sorgeranno nuove esportazioni delle nostre industrie, nuove importazioni dei prodotti australiani ed americani; in una parola, si stabilirà fra l' Italia ed i paesi "oceanici un giro importantissimo di scambi e di affari, per il quale le Galapagos saranno a noi utilissime quanto a qualunque altra nazione. Assicurato a noi il possesso di alcune di quelle isole, noi ce ne sapremo avvantaggiare senza nuocere altrui, mentre, cadute in altre mani più gelose, queste potrebbero confiscare a loro esclusivo vantaggio le utilità, che, al transito marittimo, presentano le dette isole, rendendone i beneficî pressochè insensibili alle navi italiane. 
La Floriana, punto naturale d’intersecazione, come già fu detto, del solco delle navi, che dall'Atlantico si spingeranno, nel Pacifico, attraverso il nuovo canale, è la più indicata fra tutte le isole, anche in considerazione dell'ottimo ancoraggio, che presenta alle navi, per l'impianto di uno stabilimento navale italiano.

Nessuno vi ha che non intenda di quale immenso valore nautico-commerciale sarebbe un giorno, per l'esercizio delle nostæe grandi linee di navigazione, in concorrenza con le linee similiari straniere, il possesso di uno stabilimento nazionale, al caso di provvedere, con risparmio di tempo e di danaro, a tutte le esigenze relative alla sosta dei piroscafi percorrenti le ripetute linee; crediamo non esagerare asserendo che le nostre navi si troverebbero così nel Pacifico in tale situazione da poter provarsi a reggere alla concorrenza delle navi di nazioni all'Italia superiori, per espansione di commercio, per potenza di naviglio.

Possiamo aggiungere che uno stabilimento navale italiano nell'isola Floriana risponderebbe, oltre alle necessità d'indole commerciale, altresì a due bisogni sentiti dalla nostra marina militare nelle acque del Pacifico : cioè a quello di una buona base centrale di approvvigionamento, e di un luogo di raccolta isolato per gli esercizi ed esperimenti guerreschi, adatto, nel contempo, come diporto agli equipaggi delle navi, costretti spesse volte, lungo la estesa costiera dell'America occidentale, ora per motivi politici, ora per considerazioni igieniche, a permanenze, a bordo, eccessivamente prolungate.

Posta, a parer nostro, fuor di contestazione, non che l'utilità, la necessità che l'Italia abbia nell' arcipelago delle Galapagos un luogo di sicuro approvvigionamento per le sue navi mercantili e da guerra, sorge da sè il quesito se il detto luogo debba essere di proprietà dello Stato o privata.

Se si rammenta quanto in precedenza fu detto in ordine alla condizione politica delle Galapagos, in ordine ai diritti di proprietà, che, nell'isola Floriana, luogo in cui dovrebbe sorgere lo stabilimento italiano, vantano gli eredi Villamil, non si pena a riconoscere che conviene assolutamente escludere $a$ priori ogni qualsivoglia idea di acquisto territoriale da parte del Governo.

Quello però che al Governo non riuscirebbe di fare in modo alcuno, potrebbe e dovrebbe essere fatto da un privato cittadino, o da una Società.

Un privato cittadino, una Società non porrebbero in diffidenza nè il Governo equatoriale, nè alcuno di quegli altri, i quali già hanno fermata la loro attenzione sulle Galapagos, e che possono da un momento all'altro farle acquistare tutte da qualche loro nazionale. 


\section{ISOLE Sporadi.}

È un gruppo d'isole cosi chiamato perchè le isole stesse sono sparse a grandi distanze l'una dall'altra. Compreso fra l'arcipelago di Tuamotù e la costa americana, consta di sei isole, delle quali due sole, Juan Fernandez e Pasqua (Easter degl' inglesi) sono degne di menzione.

L'isola Juan Fernandez, detta anche di Mas a tierra, assume la forma di un triangolo ottuso, il cui lato maggiore misura miglia 12.5 da N. E. e S. E. Ha un perimetro di 34 miglia, ed un'area di circa 93 chilometri quadrati. Di aspetto montuoso, mostra all'estremità N. E. una serie di colline e valli coperte di folti alberi, tra i quali s' incontrano esemplari vivi del sandalo (Santalium album?, Hook), albero che si era creduto, fino a questi ultimi anni, escinto. Il monte più alto misura 914 metri.

Nel 18\%6, la popolazione si componeva di un fattore con moglie e cinque figli, occupati nella cura del bestiame. Esistevano 100 capi di bovini, gran numero di somari e di capre.

Il terreno è ferace ed appropriato alla coltivazione di ogni specie d'ortaggi. Come traccia degli antichi abitatori spagnuoli si trovano qua e là sparsi, in grande abbondanza, il pesco, il fico ed altri alberi fruttiferi, che nascono allo stato silvestre, producendo, nonostante, eccellenti frutti.

Anni sono l' isola era frequentemente visitata da balenieri, che vi si rifornivano di viveri, legna e acqua; oggi è quasi completamente abbandonata. Affermasi che con un' intelligente amministrazione vi potrebbero vivere 10,000 capi di bestiame. La pesca potrebb'essere, nell isola, un ramo importante di speculazione: la Perca fernandesiana, e l'arigosta (Palinurus frontales, Edw.) essendo colà abbondantissime. Anche le foche, scarse un tempo, vi abbondano, notandosi la foca a doppio pelo (Otaria philippi) la cui pelle è molto ricercata.

Nell'isola Pasqua, dopo l'abbandono de' Missionari cattolici francesi, che condussero a Tahiti più della metà degli abitanti, la popolazione scese a 200 anime: 70 uomini, 25 donne e 115 bambini de' due sessi. Continuarono a rimanervi due bianchi, il signor Burguer, francese, ed un maestro d'ascia danese con la moglie chilena. 
Il signor Burguer trasformò l' isola in una vasta fattoria, facendo coltivare dagli indigeni eccellenti prodotti: lana e cuoi. Con il legname trasportato da navi, che avevano dovuto investire nell' isola in seguito a gravissime avarie, aveva fatto costruire, per uso degl'indigeni stessi, graziose casette, circondate da orti e giardini. L'isola si mantenne in comunicazione quadrimestrale con Tahiti per mezzo di una nave a vela.

Durante il soggiorno de' Missionari gli abitanti delle isole erano divisi in due frazioni: l'una, detta de' convertiti, viveva in Angorroa, e l'altra, capitanata dal signor Burguer, in Angapico. Era un continuo querelarsi e venire alle mani delle due frazioni, per ragioni di supremazia, con danno evidente della prosperità del paese. Partiti i Missionari, le cose presero miglior piega, tuttochè ricomparissero antichi diritti ed usanze, fra cui l'elezione di una regina. Questa donna, che viveva in intime relazioni con il signor Burguer, divenne l'unica autorità: ripartì il terreno fra i suoi sudditi, i quali si obbligarono a lavorare dividendo in tre parti il frutto del proprio lavoro, una parte cioè per la Regina, una per il signor Burguer e la terza per loro stessi. La sovrana riuniva ogni sera tutte le donne dell'isola in una casa costruita a tal uopo, e s' informava delle occupazioni giornaliere di ogni famiglia. Sciolta la riunione, ciascuno si dedicava a qualche particolare faccenda oppure al ballo, ritirandosi poi a casa contento ed allegro. Si ristabili un'altra carica insigne, d'indole esclusivamente ecclesiastica, ed il cui titolare doveva essere annualmente eletto in un modo stranissimo. Gli aspiranti a tale carica, consistente nel fare preci e provvedere all'umazione dei defunti isolani, si raccoglievano in un sito designato sulla punta S. O. dell' isola al principio dell'incubazione degli uccelli, ed eseguita una certa cerimonia dentro di una caverna, si lanciavano in mare, per guadagnare a nuoto l'isolotto Mutu-Raukau, distante 200 metri dalla punta. Il primo a prendere un uovo acquistava immediatamente l'alta dignità, la quale costringeva l'eletto a vivere nell'isolamento ed a farsi vedere soltanto in occasione di funebri o quando era consultato intorno ai vaticinî. 
Tabella dimostrante la posizione de' principali gruppi d'isole dell'Oceano Pacifico. ${ }^{1}$

\begin{tabular}{|c|c|c|c|c|}
\hline Nome de' Gruppi & Latitudine & Longitudine & Latitudine & Longitudine \\
\hline \multicolumn{5}{|c|}{ ISOLE DEL NORD. } \\
\hline Isole Sandwich . . . . . . . & $18^{\circ} 54^{\prime}$ & $154^{\circ} 50^{\prime}$ O.G. & $23^{\circ} 34^{\prime}$ & $164^{\circ} 32^{\prime}$ O.G. \\
\hline ». Marianne o de' Ladroni & 1224 & 14424 E. & 2030 & $1463 \mathrm{E}$. \\
\hline$»$ Pelew ....... & 653 & 13421 E. & 89 & 13455 E. \\
\hline Arcipelago Marshall . . . . . & 445 & $16522 \mathrm{E}$. & 1140 & $17230 \mathrm{E}$. \\
\hline Isole Gilbert. & 300 & $17205 \mathrm{E}$. & $143 \mathrm{SS}$. & 17745 E. \\
\hline » Galapagos. . . & 142 & $8930 \mathrm{O}$. & $123 \mathrm{~S}$. & $9134 \mathrm{O}$. \\
\hline Caroline. . . . . . . & 100 & $1121 \mathrm{E}$. & 1121 & $16252 \mathrm{E}$. \\
\hline \multicolumn{5}{|c|}{ ISOLE DEL SUd. } \\
\hline Isole dell'Ammiragliato. . . & $1^{0} 57^{\prime}$ & $146^{\circ} 49^{\prime} \mathrm{E}$. & $2^{\circ} 55^{\prime}$ & $164^{\circ} 48^{\prime} \mathrm{E}$. \\
\hline Fenici . . . . . . . . & 241 & 17180 & 437 & 174480 \\
\hline » Nuova Irlanda...... & 246 & $15033 \mathrm{E}$. & 451 & $1532 \mathrm{E}$. \\
\hline Nuova Britannia. . . . & 44 & 14817 E. & 630 & $15215 \mathrm{E}$. \\
\hline Salomone ....... & 436 & $15155 \mathrm{E}$. & 1220 & $16230 \mathrm{E}$. \\
\hline » delle Lagune. . . . . . . & 529 & $17915 \mathrm{E}$. & 1041 & 17660 \\
\hline$»$ Marchesi ........ & 753 & $13843 \mathrm{O}$ & 1030 & $14040 \mathrm{O}$ \\
\hline$»$ Santa Cruz ....... & 957 & 16541 E. & 1215 & 167110. \\
\hline Arcipelago delle Luisiadi. . & 1058 & $1513 \mathrm{E}$. & 1130 & $15426 \mathrm{E}$. \\
\hline Isole de' Navigatori. . . . & 1253 & $168 \quad 60$ & 1557 & 178210. \\
\hline » Banks....... & 1316 & $16717 \mathrm{E}$. & 1410 & $15814 \mathrm{E}$. \\
\hline » Nuove Ebridi . . . . . . & 1336 & 16640 E. & 2015 & $17011 \mathrm{E}$. \\
\hline Arcipel. Basso o di Tuamotù & 149 & 124480. & 253 & $14844 \mathrm{O}$. \\
\hline Isole Figi. . . & 1542 & $17651 \mathrm{E}$. & 1948 & 178120. \\
\hline della Società & 1611 & 14850. & 1753 & $15212 \mathrm{O}$. \\
\hline Nuova Caledonia & 1759 & $16255 \mathrm{E}$. & 2246 & 16735 E. \\
\hline Tonga . . . . . . . . & 182 & $17340 \mathrm{O}$ & 2252 & $17924 \mathrm{O}$ \\
\hline di Cook. & 185 & 157110 & 2426 & 171480. \\
\hline della Lealtà. . . . & 2025 & $16625 \mathrm{E}$. & 2232 & $1685 \mathrm{E}$. \\
\hline Sporadi & 22 & 179 & 33 & 110 \\
\hline
\end{tabular}

${ }^{1}$ Da documenti ufficiali. 
Tabella dimostrante l'area, la popolazione ed il valore delle importazioni ed esportazioni relative ad alcuni gruppi d' isole dell'Oceano Pacifico. ${ }^{1}$

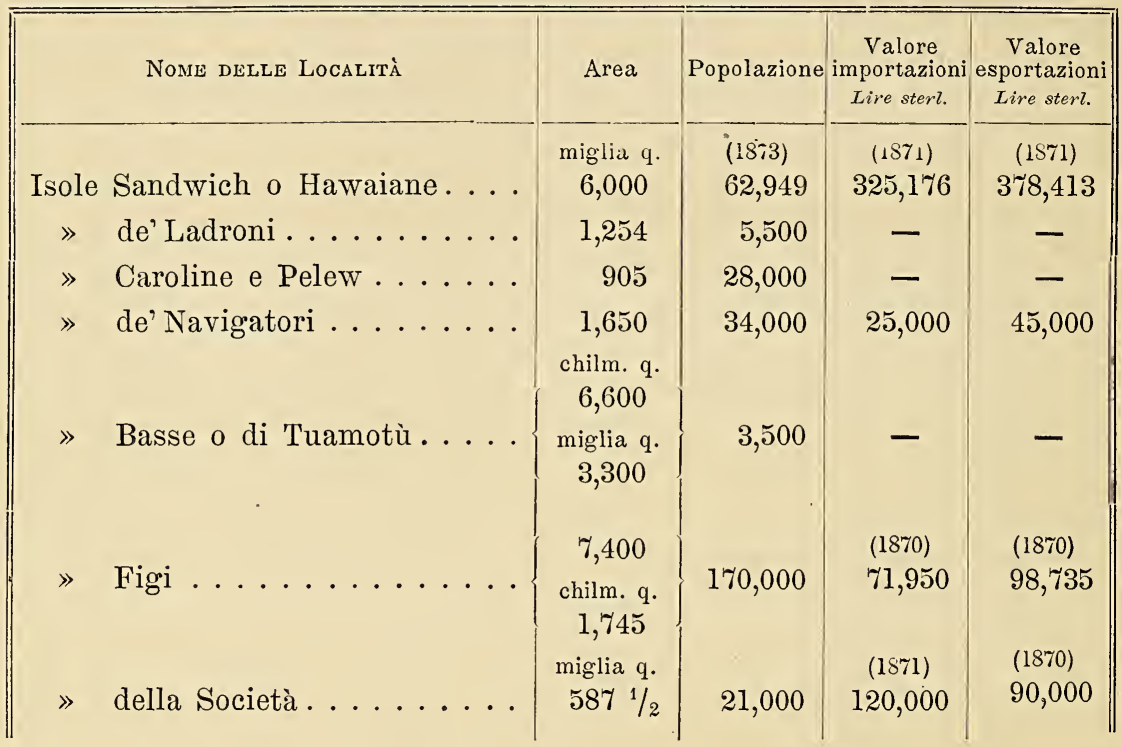

1 Da documenti ufficiali. 
ACCLIMATAZIONE DELLA SPECIE UMANA

Influenze dell'ambiente e della razza - Condizioni d'acclimatazione.

Affenmamo che la razza europea non può resistere al lavoro manuale ne' paesi intertropicali, negando così implicitamente ad essa la potenza di acclimatarvisi.

Questa nostra affermazione vuol'essere però presa in senso relativo, non già assoluto, poichè altrimenti ci porremmo, contro il nostro volere, in opposizione con i dettami della scienza antropologica, che attribuisce ad ogni razza umana la suscettibilità di adattarsi perfettamente a qualsiasi nuovo ambiente.

Tale scienza ammette, che occorrono sacrificî di vite e tempo, spesso lunghissimo, perchè l'acclimatazione avvenga laddove l'ambiente presenta condizioni di esistenza sensibilmente diverse da quelle che appartengono all' ambiente originario della razza immigrante.

Ora, non v'ha che una necessita impellente la quale possa spingere una razza ad affrontare gravi pericoli, ad esporsi al sacrificio di vite: la intangibilità della propria conservazione, la difesa contro un'imminente distruzione. 
Conseguentemente, la razza bianca, a cui la natura impone grandi sacrifici per naturalizzarsi, sotto i tropici, non si esporrà a subirli fino a tanto che essa potrá prosperare e svolgersi altrove, senza alcun danno o con danno minore.

La razza bianca potendo espandersi ancora sopra molte regioni, che non offrono alcuno de' pericoli succitati, un lungo periodo di tempo dovrá trascorrere prima che si decida ad affrontare i rischi dell'acclimatazione tropicale.

E noi saremo rimasti nel vero dicendo che le terre torride non sono attualmente, per essa, un campo di naturale esercizio delle sue facoltà fisiche, della facoltà segnatamente del braccio.

I cenni che seguono, e che sono dovuti ad uno studioso americano, di cui ci duole non rammentare, in quest'istante, il nome, valgano a chiarire meglio il nostro pensiero sull'argomento, ed a completare le ragioni del nostro lavoro sopra Tahiti e le Figi.

La specie umana, sôrta da un centro di apparizione unico, si trova in oggi sparsa ovunque. I suoi rappresentanti, nelle loro innumerevoli migrazioni, s'imbatterono nei climi più disparati, negli ambienti più opposti: dai ghiacci polari alla zona torrida.

Dovettero perciò possedere le attitudini necessarie per modellarsi secondo tutte le diverse condizioni naturali di esistenza; ossia, essere capaci di resistere a qualunque clima e di naturalizzarsi, per così dire, ovunque si trovarono.

La possibilità per l'uomo di vivere e prosperare in regioni diverse da quelle in cui hanno vissuto i suoi padri, Ifu negata, in modo più o meno assoluto, da' poligenisti. E taluni monogenisti ammisero che una razza umana costituitasi in un dato ambiente, rimaneva ad esso incatenata, non potendo cioè abbandonarlo senza perire. Da altri furono sostenute opinioni diametralmente opposte ammettendosi che un gruppo umano qualsiasi possa acclimatarsi, subito, in ogni dove.

Vi sono certamente errori ed esagerazioni in tutte codeste dottrine estreme.

Il de Quatrefages, l'illustre antropologo francese, per provare l'innocuità del cambiamento di ambiente, dimostra, contro le asserzioni del Knox, che il francese vive perfettamente in Corsica, purchè eviti i luoghi palustri del versante orientale. 
In forza della revoca dell'editto di Nantes, i fuggiaschi della Provenza, egli dice, fondarono colonie prospere nella vallata del Danubio: le razze inglese e francese, trasferitesi al Canadà, non hanno degenerato; le colonie olandesi, con i loro discendenti i Boeri, formano attualmente la repubblica di Transvaal, che ha saputo resistere contro gli eserciti inglesi.

Ciò che è una verità per la razza ariana, lo è altresì per la razza negra: il bianco ha trasportato il negro pressochè dappertutto, e l'uno vive vicino all'altro nelle regioni più lontane del rispettivo luogo d'origine. Gli ebrei sono poi veri cosmopoliti, dacchè in Prussia come in Algeria, in Europa come in America, la loro fecondità supera quella delle razze indigene.

Però, i fatti ora citati non basterebbero per poter affermare che la specie umana è suscettibile sempre di acclimatarsi, improvvisamente, in una località determinata. .

Vi sono regioni funeste per l'uomo, a qualsiasi gruppo antropologico egli appartenga, e per quanto egli possa sembrare preparato, per affrontare i loro influssi. Tali sono le località palustri, in cui le emanazioni pestilenziali, poste in effervescenza dal caldo di un clima ardente, producono endemie ed epidemie periodiche, che decimano financo gl' indigeni. Fortunatamente codesti inconvenienti possono essere corretti dall'arte, con opere opportune.

Si può asserire che ogni colonizzazione in luoghi nuovi è anzitutto una conquista tentata dalla razza immigrante; e sia che la lotta s'impegni contro l'uomo o contro l'ambiente, la vittoria non è ottenuta che al prezzo del sacrificio di vite umane.

Ogni quistione di acclimatazione comprende due termini, che sono, per dirlo con espressione meccanica, le componenti della risultante che si cerca o si studia. Questi due termini sono la razza e'l'ambiente; ma bisogna distinguere sempre bene l'ambiente normale dall'ambiente accidentalmente viziato.

Senza entrare in minuti particolari circa l'azione dell'ambiente sulle razze umane, gioverà solo indicare un fatto molto generale, che interessa al più alto grado il progresso dell'acclimatazione.

È saputo che le razze animali, i vegetali della stessa specie, tuttochè non tralascino di essere, nel fondo, accessibili alle stesse influenze, hanno attitudini loro proprie; e che tale affezione, molto frequente in una razza od in una specie, sarà, al contrario, molto rara in un'altra razza od in un'altra specie. Accade esattamente lo stesso circa la specie umana.

I miasmi palustri operano nello stesso modo sopra tutti gli uomini.

Il negro soffre e muore di febbre sulle sponde del Niger, ma soffre con minore intensità ed il numero dei decessi è meno considerevole fra i negri che fra i bianchi: inoltre, le due razze trasportate nelle Indie, l'europea e 
la negra, si mantengono a detto riguardo, su per giù, nelle stesse proporzioni. Paragonato con le altre razze, il negro gode di un vantaggio, quello di essere meno colpito dalle emanazioni palustri. Nato in una regione in cui le respira in ogni dove, avendo per antenati chi visse fin da' tempi preistorici in quell'aria mefitica, si trova a preferenza di ogni altro uomo acclimatato a cotale ambiente; epperò prospera, senza stento, colà dove il bianco sarà esposto a soffrire durante lungo tempo.

Ma il negro ha il petto delicato, e nessun'altra razza è più della sua soggetta alla tisi.

Dalle differenze estreme che presentano fra loro il negro, ed il bianco europeo, risulta che le condizioni generali di acclimatazione si manifestano opposte per queste due razze.

Pertanto, un'aria alquanto calda impregnata di emanazioni palustri è deleteria per l'europeo; un freddo umido, quantunque moderato, uccide il negro.

Questi fatti mostrano che le condizioni di acclimatazione variano da una razza all'altra; che lo stesso ambiente non può esercitare lo stesso genere di azione sopra razze diverse, e che l'acclimatazione completa, la naturalizzazione, non può risultare se non da' seguenti due termini: razza ed ambiente.

Secondo il de Quatrefages riesce facile il dimostrare, col riferirsi a quanto accade fra gli animali e le piante, che l'organismo talune volte si modifica, piegandosi alle esigenze di un ambiente quasi inflessibile di sua natura.

I crisantemi originari della Cina, trasportati in Francia, nel 1790, vi fiorirono e produssero frutti senza giungere a maturità. Solo, nel 1852, alcuni steli fiorirono e fruttificarono meglio degli altri: maturarono i semi, ed oggigiorno la pianta ne produce a sufficienza. Un piccolo numero di steli accidentalmente precoci acclimatò adunque quel grazioso fiore in Francia.

La storia dell'oca egiziana è più notevole ancora.

Importata in Francia nel 1841 da Géoffroy de Saint-Hylaire, questa specie covava sul principio nel mese di dicembre, come nel suo paese nativo. I suoi pulcini venivano alla luce in pieno inverno, e quindi in condizioni poco favorevoli. Poco a poco, la covatura andò ritardando; ebbe luogo in febbraio nel 1844, ed in aprile nel 1846, epoca della covatura dell'oca francese.

Egli è evidente che l'organismo dell'oca egiziana si è accomodato alle condizioni imposte dal clima di Francia. Perciò l'adattamento fisiologico ad un nuovo ambiente è un fatto incontestabile. Ma codesta armonia non si ottiene mai senza sacrifici; ed a cotesto riguardo l'uomo rassomiglia agli animali ed alle piante.

Si richiesero più di venti anni perchè l'organisıno delle oche e dei gallinacei europei si mettesse in armonia con le condizioni di esistenza imposte dagli altipiani americani. 
L'adattamento al nuovo ambiente, come fu detto, non si acquista che a prezzo del sacrificio di un certo numero d'individui e di generazioni.

In Algeria, ne' primi tempi della conquista, la lotta del bianco contro l'ambiente fu delle più violenti e micidiali, ma, nel 18\%, cioè dopo 40 anni, la popolazione di razza europea presentò un aumento di 25,000 anime, dovuto esclusivamente all'eccedente del numero delle nascite su quello de' decessi.

Si osservarono però delle differenze, sotto il punto di vista della facilità di acclimatazione, tra le diverse razze europee; ad esempio, fra gli abitanti del Nord e del Mezzodì della Francia. I corsi, i provenzali, gli spagnoli, i maltesi si acclimatarono più facilmente degli altri europei.

Si è molto discusso intorno alla possibilità, per l'europeo, di acclimatarsi nei grandi arcipelaghi delle Antille, ove la febbre gialla è per lui cotanto micidiale. Il de Quatrefages non crede che l'esperienza sia completa ancora; ed a suo avviso, se la razza francese non si è del tutto acclimatata nella Martinica, e nella Guadalupa, lo sarà un giorno. Appoggia questa sua opinione sulla longevità e la fecondità notevoli che il Simonot ha colà constatato. L'acclimatazione del negro, nelle Antille francesi come nelle ingleși, ha dovuto necessariamente essere inceppata dalle condizioni della vita sociale.

Il negro è stato importato in quei paesi dalla violenza e vi ha vissuto fino a questi ultimi anni nella condizione di schiavo. Dopo l'abolizione della schiavitù, dice Elisée Reclus, la popolazione negra nelle isole inglesi è in via di aumento.

L'ambiente, la natura della razza, non costituiscono il tutto, nei complessi problemi della acclimatazione. L'uomo, l'individuo stesso, v'introducono i loro elementi proprî. Il selvaggio e l'europeo moderno si trovano collocati, per il solo fatto della differenza sociale che li separa, in condizioni talune volte opposte, e che non sono sempre a favore di quest'ultimo.

La civiltà moderna, per i suoi agi come per i suoi vizî, ci rende meno atti ad affrontare le eventualità dell'acclimatazione.

Nell' isola di Bourbon, tenuta come una delle località più funeste per gli europei, i registri della mortalità, considerando la popolazione nel suo insieme, accusano un eccedente formidabile dei morti in confronto delle nascite; ma decomponendo questo eccedente si riscontrano in realtà due classi o razze distinte, per usi e costumi. L'una comprende gli abitanti della città e delle case ricche, che non lavorano mai alla coltura della terra; l'altra, i petits blancs che, oltremodo poveri, per procurarsi il lusso di possedere schiavi, si vedono obbligati a lavorare la terra, con le proprie braccia. La prima di queste due classi è quella che offre cifre più elevate sul registro delle mortalità.

La razza de'petits blancs si conserva perfettamente e le donne, in par- 
ticolar modo, richiamano l'attenzione per la bellezza delle forme e del sembiante. Egli è che nell' isola Bourbon, mentre l'ozio ed il genere di vita che mena il ricco uccidono lui e chi lo imita, il povero si acclimata, grazie alla sobrieta, all'abitudine e ad un lavoro moderato.

Per se stesso, questo fatto dev'essere agli occhi dell'antropologo e di tutti, un serio ammaestramento scientifico ed economico.

L'acclimatazione o naturalizzazione è sempre possibile purchè si subiscano i sacrificî ch'essa impone; quindi, tutte le razze umane possono vivere e svilupparsi più o meno bene in tutti gli ambienti non visitati da cause accidentali.

Dietro gli ammaestramenti dell'esperienza, che si rende giornalmente palpabile, ed i fatti confermati dalla storia, il de Quatrefages esprime nei seguenti termini l'idea generale ch'egli si è formata, del modo onde si è popolato il mondo:

« La razza ariana c'insegna da sè sola la storia della specie umana.

« Noi la vediamo uscire dal Bolor e dall' Indokoh (Paropamisus), dove l'estate non dura che due mesi, poi scendere dalla Boukkaria (Sogdiane), percorrere la Persia ed il Cabul, arrivare nella conca dell' Indo. Undici stazioni segna questa marcia seguita dagli ariani prima di giungere al Gange. Da questo punto la vediamo andare innanzi, passo a passo, distaccando avanguardie, per preparare le sue conquiste.

«Oggi la razza bianca si trova sotto i tropici, nelle Indie, sotto il circolo polare, nella Groenlandia, ricopre un'immensa zona di climi più o meno temperati, possiede colonie in ogni dove.

« La specie umana, ne' suoi primordî, ha dovuto procedere come gli ariani. Nell'uscire dal suo centro di creazione procedè di tappa in tappa lentamente, a fine di poter conquistare il mondo deserto. In tal guisa si sarà, grado a grado, abituata alle diverse condizioni di esistenza impostele dal Nord e dal Sud, dall' Est e dall'Ovest, dal freddo e dal caldo, dalla pianura e dal monte. Divergendo in tutti i sensi ed imbattendosi in ambienti differenti, la specie umana si sarà posta, gradatamente, in armonia con ciascuno di essi.

«L'acclimatazione, camminando così di pari passo con le conquiste geografiche, sarà stata meno micidiale; e la lentezza della marcia avrà reso la lotta meno penosa.

«Molti saranno stati i caduti, ma i superstiti, non avendo dinanzi a sè che la natura, andarono avanti e popolarono il mondo ».

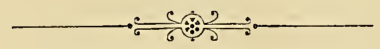




\section{N D I CE}

T A H I T I.

Capitolo I. - Reminiscenze di Tahiti:

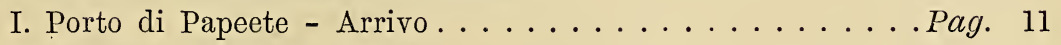

II. Le feste della Repubblica . . . . . . . . . . . . . . . 13

III. Visita della Regina alla Caracciolo . . . . . . . . . . . . . . . 16

IV. Una colazione canaca . . . . . . . . . . . . . . . . . . 19

V. Gita all' isola di Moorea. . . . . . . . . . . . . . . . . . . . . . . . . . . .

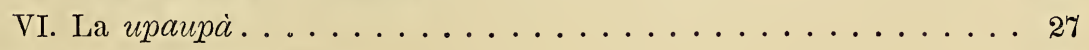

VII. La pesca del tonno. . . . . . . . . . . . . . . . . . . . 29

Capitolo II. - Cenni storici:

I. Religione, usi e costumi . . . . . . . . . . . . . 33

II. Scoperta della Polinesia - Conseguenze . . . . . . . . . . . . . . . . . . . . . 41

Capitolo III. - Lingua della Polinesia. . . . . . . . . . . . . 45

Capitolo IV. - Ordinamento politico:

I. Divisione politica . . . . . . . . . . . . . . . . 49

II. Protettorati - Annessioni - Prese di possesso . . . . . . . . 50

III. Ordinamenti indigeni sotto il Protettorato. . . . . . . . . . 52

IV. Ordinamenti coloniali e indigeni dopo l'annessione . . . . . . . 55

Capitolo V. - Produzione economica:

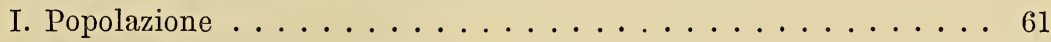

II. Prodotti agricoli - Pesca. . . . . . . . . . . . . . 65

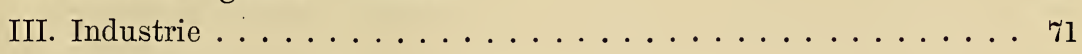

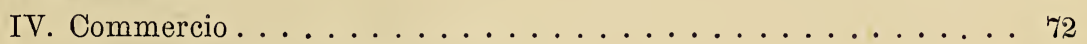

Viaggio di circumnavigazione. Vol. II 35 
Capitolo VI. - Geologia di Tahiti:

I. Cenni sulla costituzione fisica dell'isola . . . . . . . . Pag. 77 CApitolo VII:

I. I coralli. ..................... 8 .

Capitolo VIII. - Fauna e flora:

I. Visita all'atollo Takumé - Dragaggi nelle vicinanze di Tuamotù. 93

II. Cenni generali sulla fauna terrestre, avifauna e fauna marina . . 95

III. Escursione botanica del Dottor Rho . . . . . . . . . . . . . . 98

IV. Moorea - Maggiori particolari sulla flora polinesiana. . . . . . 104

V. Il botanico Bertero. . . . . . . . . . . . . . . . . . 108 Capitolo IX:

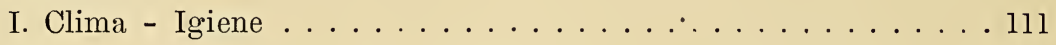

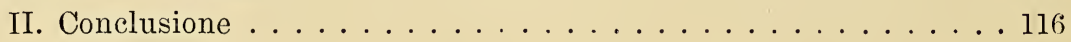

F I G I

Capitolo I. - Reminiscenze:

I. Approdo alle Figi - Il Capitano Ferrea . . . . . . . . Pag 121

II. Suva - Lami . . . . . . . . . . . . . . . . . 123

III. Escursione a Nausori ed a Rewa - L'agente consolare italiano -

Partenza della Caracciolo................ 127

IV. Stabilimento di Nausori della Colonial Sugar Refining Company

di New South Wales e Figi. . . . . . . . . . . . 131

Capitolo II. - Cenni storici:

I Confine dell'arcipelago delle Figi - Sua scoperta - I primi bianchi. 137

II. Il figiano e la sua pretesa origine . . . . . . . . . . . . . 140

III. Mitologia - Religione . . . . . . . . . . . . . . . . . . . 142

IV. Indole del figiano; suoi antichi costumi e leggi . . . . . . . . . 146 Capitolo III. - Ordinamento politico:

I. Annessione delle Figi ai dominî britannici. . . . . . . . . . . . 153

II. Governo coloniale inglese moderno - Ordinamenti governativi per

gli europei e per gli indigeni. . . . . . . . . . 160

Capitolo IV. - Cenni etnologici:

I. Popolazione indigena - La rosolia - Educazione e religioni moderne. 169

II. Cerimonie funebri del passato e del presente. . . . . . . . . . 174

III. Industrie indigene . . . . . . . . . . . . . . . . . 182 Capitolo V:

I. Agricoltura moderna . . . . . . . . . . . . . . . . . . 189

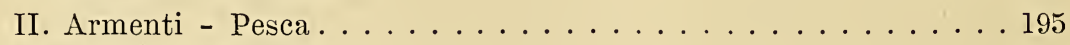

III. Industrie moderne $\ldots \ldots \ldots \ldots \ldots \ldots \ldots \ldots$

IV. Commercio . . . . . . . . . . . . . . . . . . . . . . . . . 199 


\section{Capitolo Vi:}

I. Geologia - Orografia - Topografia............. Pag. 205

II. Clima - Meteorologia . . . . . . . . . . . . . . . . . . . . . . . . . . . . . . . . . . . . . . . . . . . . .

III. Flora e fauna . . . . . . . . . . . . . . . . . . . . . . . . . . . . . . . . . . . . . . . . . .

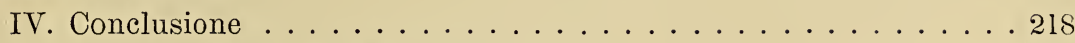

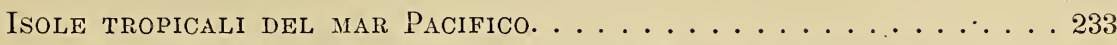

Isole dell'Oceania che si trovano fuori della sfera d'influenza politicoeconomica di Tahiti e delle Figi. . . . . . . . . . . 248

Acclimatazione della specie umana. - Influenze dell'ambiente e della

razza - Condizioni d'acclimatazione . . . . . . . . . 267

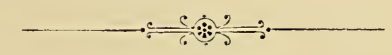







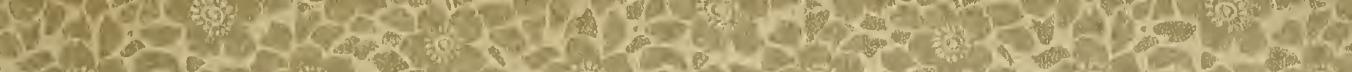

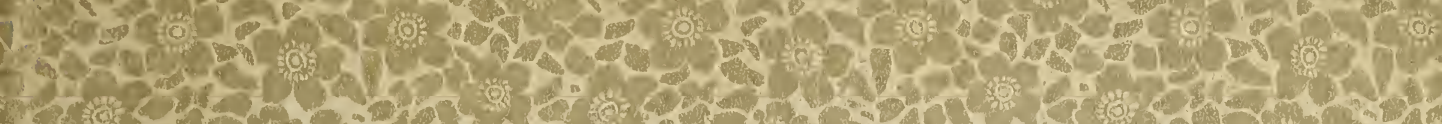

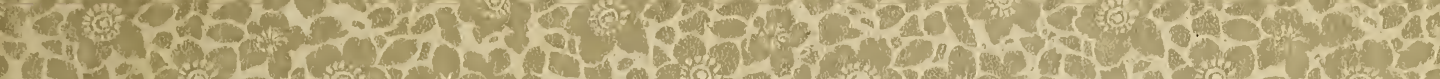

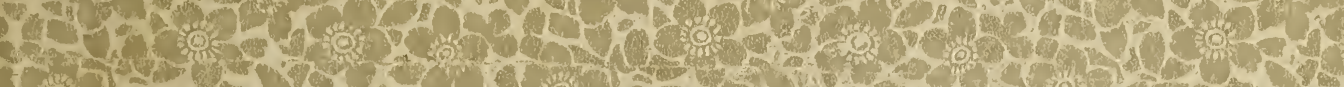

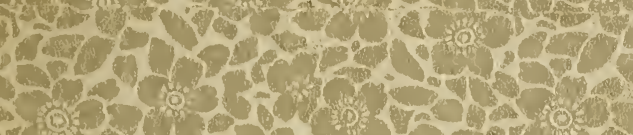

ats the 3 mo

1.

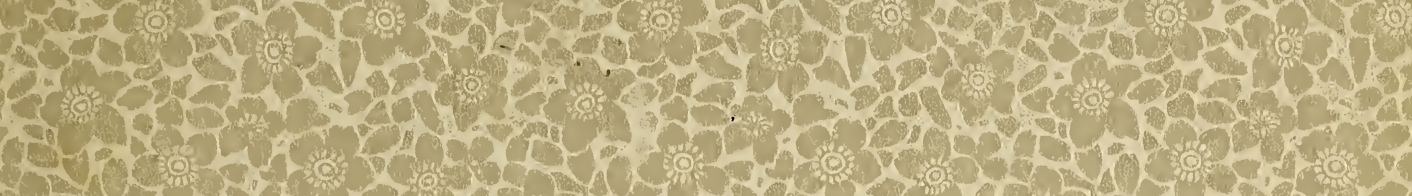

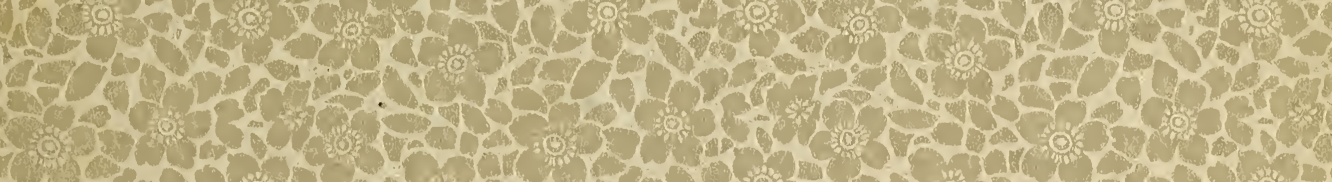

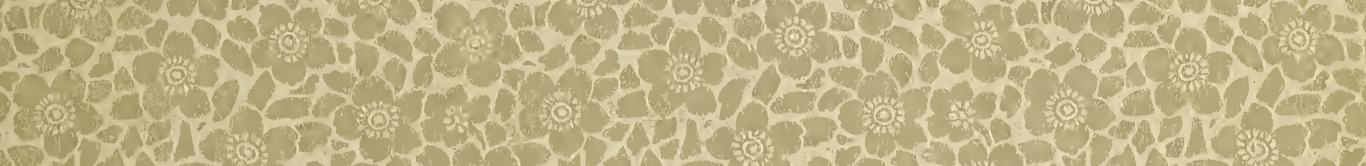

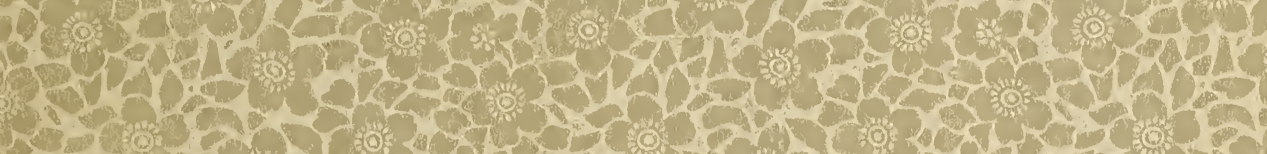

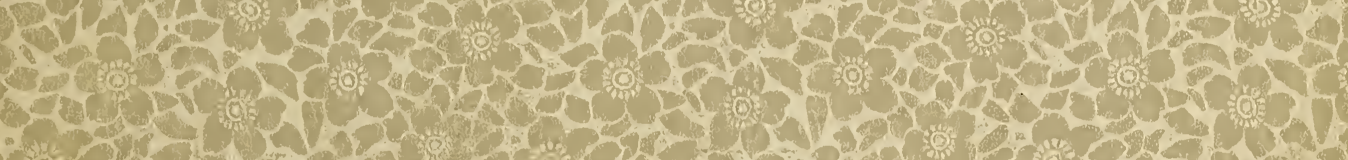

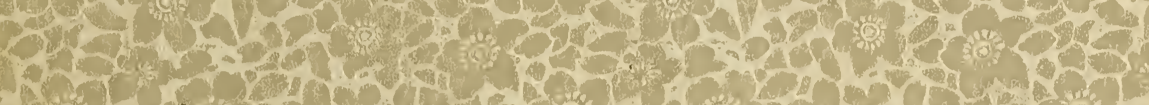

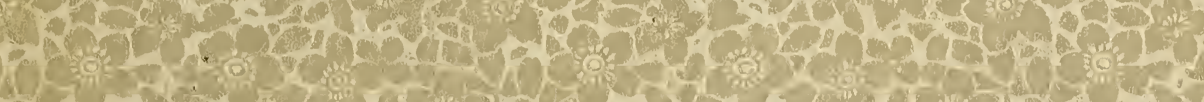

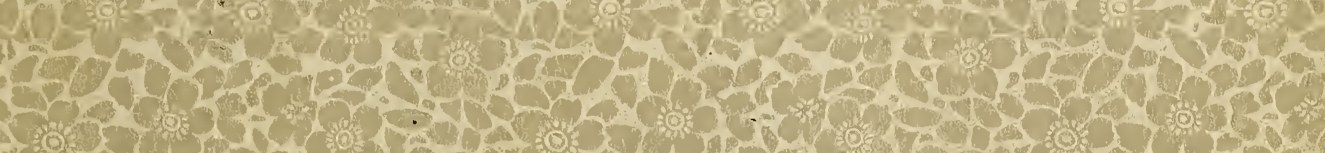

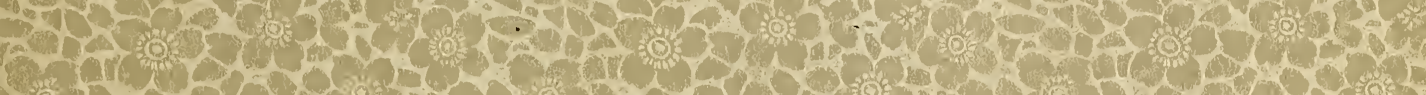

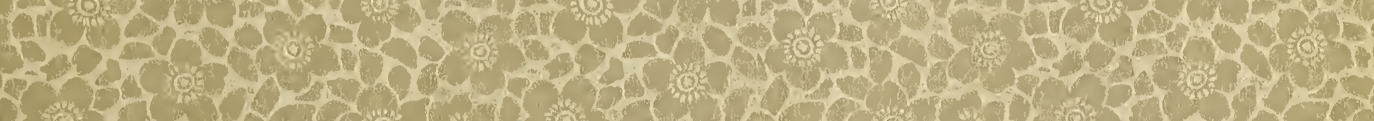

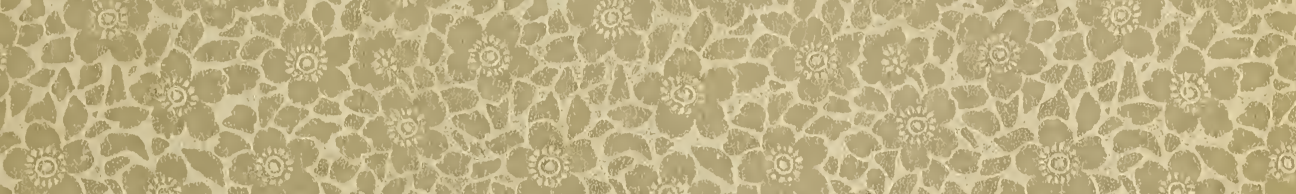

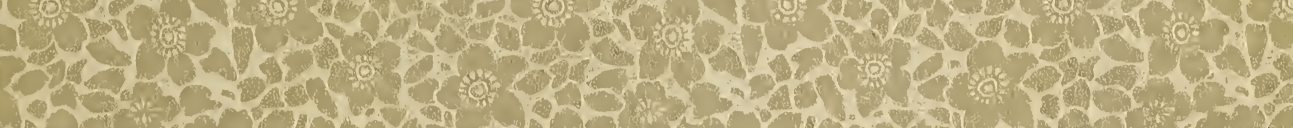
A.

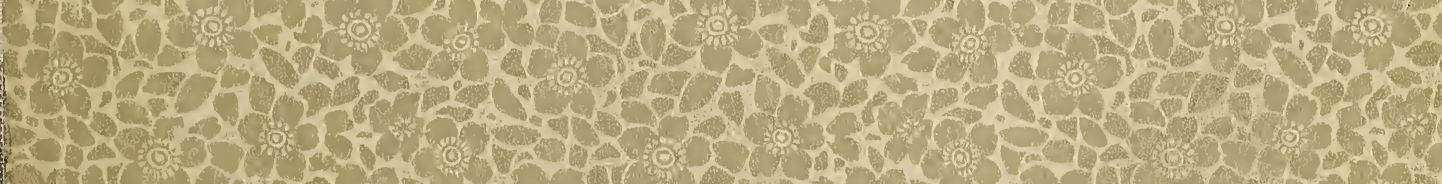
10

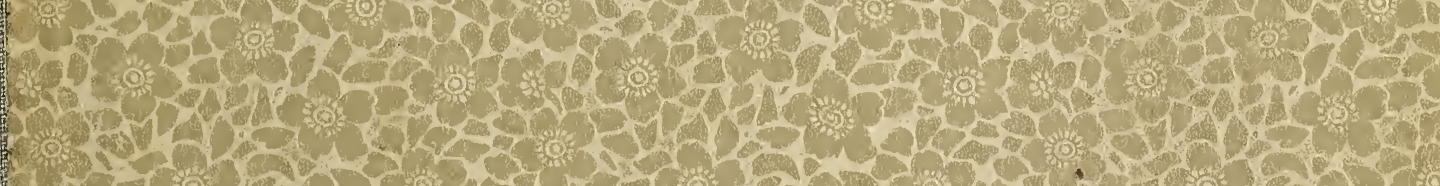

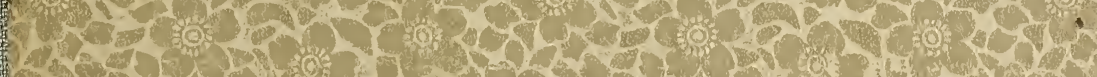

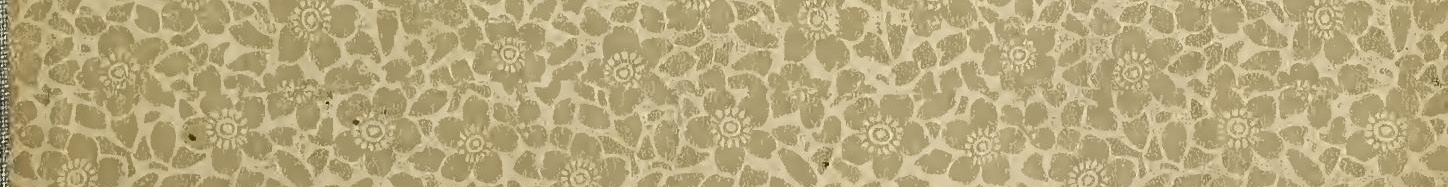
S. 
